\title{
Caddo Pottery from Eight Sites in the Middle Ouachita River Valley
}

\author{
Mary Beth D. Trubitt
}

Arkansas Archeological Survey, HSU Research Station

Follow this and additional works at: https://scholarworks.sfasu.edu/ita

Part of the American Material Culture Commons, Archaeological Anthropology Commons, Environmental Studies Commons, Other American Studies Commons, Other Arts and Humanities Commons, Other History of Art, Architecture, and Archaeology Commons, and the United States History Commons

Tell us how this article helped you.

This Article is brought to you for free and open access by the Center for Regional Heritage Research at SFA ScholarWorks. It has been accepted for inclusion in Index of Texas Archaeology: Open Access Gray Literature from the Lone Star State by an authorized editor of SFA ScholarWorks. For more information, please contact cdsscholarworks@sfasu.edu. 


\section{Caddo Pottery from Eight Sites in the Middle Ouachita River Valley \\ Creative Commons License \\ (c) (1) \& 8}

This work is licensed under a Creative Commons Attribution-NonCommercial 4.0 International License 


\title{
Caddo Pottery from Eight Sites in the Middle Ouachita River Valley
}

\author{
Mary Beth Trubitt \\ Arkansas Archeological Survey, HSU Research Station
}

Documentation and analysis of ceramic vessels in the Joint Educational Consortium's Hodges Collection has focused on reconstructing grave lots based on notes left by amateur archeologist Vere Huddleston in the 1930s and 1940s. Despite problems with the data, we can glean useful information from this collection. Here, I describe Caddo pottery and other artifacts in grave lots from eight sites in Clark and Hot Spring counties of west-central Arkansas. I then order the grave lots in time based on stylistic and technological characteristics (seriation) to refine the ceramic chronology of the Middle Ouachita River valley and compare mortuary assemblages through time and across space.

\section{Introduction}

The Joint Educational Consortium (JEC) of Henderson State University and Ouachita Baptist University owns a major collection of Native American artifacts accumulated in the 1930s and 1940s by Thomas and Charlotte Hodges from archeological sites near their home in Bismarck, Arkansas. Since the JEC acquired the Hodges Collection in 1977, archeologists at the Arkansas Archeological Survey's research station at Henderson State University (ARAS-HSU) have curated, researched, exhibited, and interpreted the collection. This article continues our efforts to document artifacts in this collection and publish our research results (Early 1986, 2012; Trubitt 2016a, 2016b, 2017; Trubitt and Evans 2015).

Thomas and Charlotte Hodges, along with Vere Huddleston, Robert Proctor, and Charles Richardson in Arkadelphia, were collectors and amateurs active in the development of Caddo Area archeology in the mid-twentieth century (Girard et al. 2014:8-16). The Hodgeses purchased Huddleston's collection of about 350-400 pieces of pottery and other artifacts after his death in 1946 (Hodges and Hodges 1945). Included with the collection were his notebooks and manuscripts. Since Huddleston kept somewhat better records of the provenience of artifacts than the others, we have been able to reconstruct grave lots he dug at a number of sites in Clark and Hot Spring counties, including East Place, 3CL21 (Trubitt and Evans 2015).

From a research standpoint, grave lots provide information about mortuary offerings and sets of artifacts that were deposited contemporaneously, even if they were not necessarily all made or used at the same time or in the same locality. Grouping and ordering grave lots based on similarities in artifact styles (seriation) is a time-tested archeological method of building a chronological sequence and has been used as such in the Middle Ouachita River valley. For example, in his 1970 dissertation Frank Schambach (1998:124-128) redefined the Mid-Ouachita phase based on five ceramic lots from graves and special deposits previously excavated at the Means (3HS3) site. Ann Early (1993) defined additional phases for the Middle Ouachita River valley based on grave lot seriation from salvage excavations at Hardman (3CL418) in advance of highway construction. Her definitions of the East (A.D. 1100-1350), Mid-Ouachita (A.D. 13501500), Social Hill (A.D. 1500-1650), and Deceiper (A.D. 1650-1700) phases form our current regional chronological framework for the Caddo period (Early 1993, 2002a, 2002b, 2002c, 2002d).

In this study, I describe ceramic vessels and other artifacts from grave lots dug by Vere Huddleston from eight archeological sites near the confluence of the Caddo and Ouachita rivers in west-central Arkansas. Documentation of vessels and examination of old records from these sites has been part of ARAS-HSU efforts to advise the JEC and the Caddo Nation in their ongoing consultations about the Hodges Collection. In 2017, the JEC transferred human remains and associated funerary objects identified in the Hodges Collection to the ARAS-Coordinating Office in Fayetteville for inventory and notifications as required under the Native American Graves Protection and Repatriation Act (NAGPRA). Materials from four of the sites discussed 


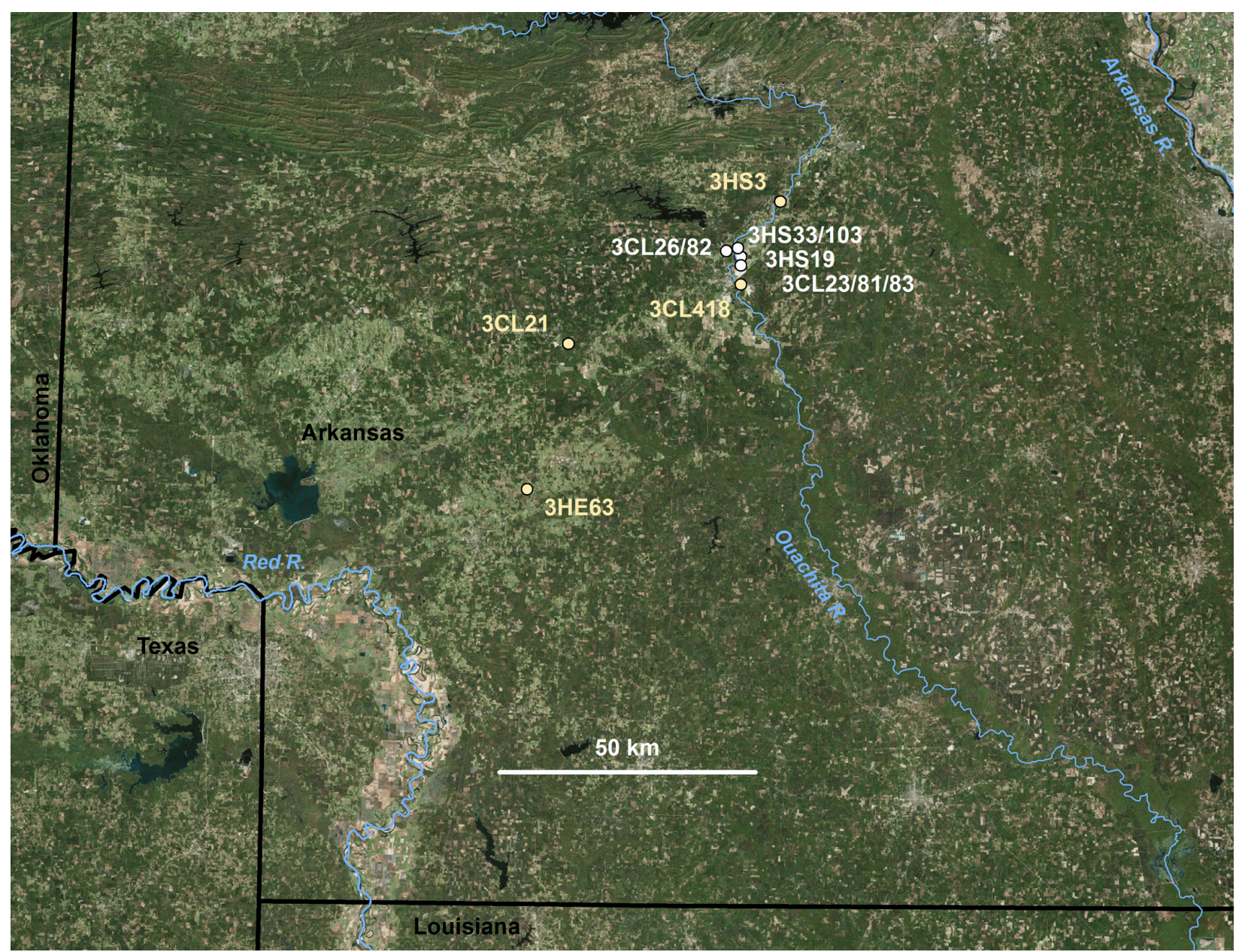

Figure 1. Location of sites in this study and other sites in southwest Arkansas mentioned in the text (base map: ArcGIS World Imagery, ESRI 2014).

here (Lower Meador, 3HS19; Upper Meador, 3HS33; Stanford, 3CL81; and Richardson, 3CL83) were part of that transfer, and this documentation will contribute to the NAGPRA notifications for those sites.

\section{Huddleston's Work at Eight Sites}

Vere Huddleston's records describe his work between 1937 and 1944 at eight sites along the Caddo and Ouachita rivers in Clark and Hot Spring counties

(Figure 1). Now recorded as separate archeological sites, several of these were close enough in space and time to have been part of the same ancestral Caddo Indian communities. Huddleston described Trigg (3CL26) and Cook (3CL82) as about half mile $(0.8 \mathrm{~km})$ apart, while Upper Meador or Upper Meadow (3HS33) and Ashby (3HS103) were quarter mile $(0.4 \mathrm{~km})$ apart. Stanford (3CL81), Richardson (3CL83), and Rorie (3CL23) were within half mile $(0.8 \mathrm{~km})$ of each other. All eight sites are situated in an area of about 5 square miles (12.9 square $\mathrm{km}$ ).

Huddleston and the other amateurs were not skilled excavators, nor were they systematic in their notetaking. There are vessels described but missing from the JEC Hodges Collection, either because Huddleston did not collect pots that were too fragmented to reconstruct, or because "finds" were sometimes divided between the people in the group on a given day. Huddleston kept a numbered list of locations he visited. In 1937, he described the graves he probed and dug, sometimes with sketches showing positioning of grave goods. He gave catalog numbers (from 1 into the 200s) to artifacts he saved from graves; he (?) later re-numbered these artifacts (1100s to 1400s) and inked these numbers on the vessels themselves. The inked numbers are not in sequence with dates of his site visits. 
For some of these artifacts, there are catalog cards with descriptions, measurements, and sketches, but we do not have a surviving catalog.

In 1939, Philip Phillips, then finishing his Harvard University dissertation on Mississippi River valley archeology, made a survey of sites in the Ouachita River valley. He photographed vessels in the collections of Thomas and Charlotte Hodges and Vere Huddleston at that time (there is only a passing mention of this region in his dissertation [Phillips 1939:202]). After the JEC acquired the Hodges Collection in 1977, Ann Early used copies of the Phillips photographs and photo cards (on file at ARAS-HSU) to identify the proveniences of many of these vessels and link Huddleston's locations to recorded archeological sites.

Huddleston's numbering system changed in 1939 (as a result of his conversations with Phillips?) and there was a new sequence of numbers in the notes and on the pots themselves (2000s series, in sequence by excavation date). At some point Huddleston also mimeographed a sketch of a human skeleton and added north arrows, artifact placement sketches/numbers/ notes, and site/grave numbers by hand during his site visits. A typescript describes sites, graves, and artifacts illustrated in some instances with Phillips's 1939 photos (copies of Huddleston's notebooks are on file at ARASHSU). Lacking from Huddleston's notes (and those of his contemporaries) are maps of the sites themselves or the spatial arrangement of graves relative to other site features. While the Hodgeses left a handwritten catalog with descriptions of artifacts, very few sketch maps have been found in either the Hodges (or Huddleston) notes. Vessels and other artifacts in the JEC Hodges Collection were accessioned in 1977 (ARAS accession number 77-1) and coded with numbers representing site and object (e.g., 2-5, where 2 codes for site 3HS19 and 5 codes for a specific artifact from that site).

Trigg (3CL26) and Cook (3CL82)

Two graves were dug at Trigg Place or Triggs Mound (3CL26) by Huddleston (his site 19) with fellow amateur Dr. Ritchie in February 1941. Huddleston's notes described a mound at Trigg as "about fifteen feet high and cone shaped." He indicated that Ritchie had dug there previously (vessels from several collectors form part of Henderson State University [HSU] Museum collections from 3CL26). He also mentioned that Trigg had been visited "by a Smithsonian Institute representative" (this would have been Edward Palmer in 1883 [Jeter 2009:290, 299]); river overflow and erosion had already impacted Trigg by this time (Thomas 1894:247). Three vessels in the JEC Hodges Collection can be linked to Huddleston's work at Trigg (77-1/29, Table 1).

Cook Place or Cook Farm (3CL82) was described as "a small rise almost in the center of the field" where "[t]he graves are shallow from 12 to 24 in deep and all prone facing west" at the time of Huddleston's visits in April 1939 (site 35 in his notes). Huddleston dug five graves at the site, accompanied on different days by Dr. Ritchie, Clara Joyce, Dr. Whaley, "W.S." and "Dub" (Huddleston occasionally made notes about the graves dug by the others). HSU Museum collections include 10 vessels from Whaley's, Proctor's, and Richardson's efforts at the site. A total of 13 vessels in the JEC Hodges Collection (and one vessel in HSU Museum collections) have been identified to Huddleston's five grave lots from Cook (77-1/6, Table 1).

Trigg and Cook are recorded in the Arkansas site file database, but with imprecise locations. Neither has been visited by professional archeologists in the modern era.

Upper Meador (3HS33) and Ashby (3HS103)

Huddleston's trips to Upper Meador or Upper Meadow (3HS33, his site 9 or 9a) took place between 1940 and 1943. Burials were dug in the mound (described as "upper mound") by Huddleston, as well as by Proctor and Richardson (45 of which are included in HSU Museum collections). Huddleston described seven graves. A total of 15 vessels and eight arrow points in the JEC Hodges Collection (77-1/4, Table 2) have been linked to these graves and another vessel from one of the Huddleston graves is in HSU Museum collections. Human remains (identified as "Meadow"), possibly from 3HS33, have been transferred to Fayetteville for NAGPRA documentation.

Huddleston visited Ashby Farm or Ashby Mound (3HS103, his site 86) and described the site as "a 


\begin{tabular}{|c|c|c|c|c|c|c|c|c|}
\hline \multirow[b]{2}{*}{ Grave Information } & \multicolumn{2}{|c|}{ Huddleston'Hodges } & \multicolumn{2}{|l|}{ ARAS ARAS Documentation: } & \multirow[b]{2}{*}{ Surface/Decoration } & \multirow{3}{*}{ Descripitive } & \multirow{2}{*}{ Temper } & \multirow[b]{2}{*}{ Comments } \\
\hline & Cat. \# & Cat. \# Huddeston Description & Art. No. Yessel Form & Type/Variety & & & & \\
\hline \multicolumn{8}{|l|}{ 3CL26, Trigg (Huddleston's Site 19): } & \\
\hline \multirow{2}{*}{\multicolumn{2}{|c|}{$\begin{array}{l}\text { Burial X, no trace bone, 2/23/1941 } \\
\text { [Mil-Ouachita - Social Hill phase] }\end{array}$}} & 2464 small bottle & $29-2$ bottle & Blakely Engraved var. Witherspoon & engraved & plain : : Emil 6 & shell + grog & vertical neck, 2464 inked on side \\
\hline & & 2465 large flared bowl & 29-3 jar,short rim & & incised & Andes $4:$ : Bard 8 or Altus 2 & shell & sooting, 2465 inked on base \\
\hline \multicolumn{2}{|l|}{ Burial Y, notrace bone, 2/23/1941 } & 2466 small food jar & & & & & & \\
\hline \multicolumn{2}{|l|}{ [Mid-Ouachita phase] } & 2467 tall seed jar & $29-1$ sed jar & & plain & & shell + grog & partial vessel, 2467 inked lower body \\
\hline \multicolumn{9}{|l|}{ 3CL82, Cook (Huddleston's Site 35): } \\
\hline \multirow{6}{*}{$\begin{array}{l}\text { Grave 1, on small rise, skull could not be save } \\
\text { 4i9/1939 } \\
\text { [Social Hill phase] }\end{array}$} & 174 & cazucla & & & & & & \\
\hline & 175 & vase & & & & & & \\
\hline & 176 & 1366 cazucla & 1-1366 in HSU Mus Coll attrib to $3 \mathrm{H}$ & 19 Sandford Punctated & punctated & Codar 1: plain & $\operatorname{grog}$ & Phillips photo 3850 (Hudd 176) \\
\hline & 177 & celt & & & & & & \\
\hline & 178 & 1359 small vase & 6-13 jar,tall rim & Foster Trailed-Incised var: Dobson & incised/brushed & Arlis 3 (type) :: Baker 1 (typec) & shell & sooting, 1359 inked; Phillips phoo: 3876 (Hudd 178) \\
\hline & 179 & 1243 water bottle & 6-1 bottle & Keno Trailed var. Curris & incised & plain: : Belhaven 17 & shell & bulbouss spool, pedestal, 1243 ink, Phil 3922 (Hudd 179) \\
\hline \multicolumn{9}{|l|}{ Another grave, dug by Dr. Ritchic 49/1939 } \\
\hline \multirow{6}{*}{$\begin{array}{l}\text { Grave 2,20 E of md otr, bone in poor shape } \\
\text { 4.9/1939 } \\
\text { [Social Hill-Deceiper phase] }\end{array}$} & 180 & 1357 vase & 6-12 jar,tall rim & Foster Trailed-Incised var: Foster & incised & Alfired 6 (type) : : Baker 3 (type) & shell & sooting, 1337 inked; Phillips photo $3948-49$ (Hudd 181) \\
\hline & 181 & 1358 vase perfect- sketched & 6-10 jar,tall rim & Foster Trailed-Incised var: Foster & incised & Alfired 2 (type) : : Baker 16 (type) & shell & sooting, 1358 inked; Phillips photo 3959 (Hudd $180[181]$ ) \\
\hline & 182 & small vase, perfect, incised, sketched & & & & & & \\
\hline & 183 & small vase & & & & & & \\
\hline & 184 & $1369 \mathrm{w}$ bottle Glendora ware sketched [prob. 6-11] & 6-11 bottle & Keno Trailed var. Red Hill & incised & plain : : Belhaven 31 & sholl & spool, pedestal; probe holes; Phil 3855 (Hudd 84 [184]) \\
\hline & 185 & 1354 crushed cazuella cone shaped & $6-8$ bovl,carinated & Means Engraved & engraved & Enos 19 (type) : : plain & shell + grog & 1354 ink, Phil 3891 (Hudd 185); Suhm \& Jelks 1962:Pl.533. \\
\hline \multirow{4}{*}{$\begin{array}{l}\text { Crave 3, child \& 2nd grave nearby, skull } \\
\text { 4//10/1939 } \\
\text { [Deceiper phase] }\end{array}$} & 186 & 1367 offset rim bowl perfect, sketched & 6-3 bowl, carinated, compound & Belcher Engraved & engraved & Central or Era: : plain & shell & probe hole, 1367 inked; Phillips photo 3910 (Hudd 186) \\
\hline & 187 & 1364 small vase perfect & $6-9$ jar,short rim & Karnack Brushed-Incised var. Miduay & incised & plain : Abraham 1 (type) & shell & 1364 inked on base; Phillips photo 3874 (Hudd 187) \\
\hline & 188 & 1361 water bottle perfect teched $w$ red pain, sketch & & Hodges Engraved var. Hodges & & & & \\
\hline & 189 & $\begin{array}{l}1362 \text { large bowl perfect, over skull, sketched } \\
\text { string of shll beads } \\
\text { part of a large pot }\end{array}$ & 6-6 bow,simple & Keno/Wallace? & incised & plain : Buffalo 5 & shell & "nelmet' shape, 1362 inked; Phillips photo 3993 (Hudd 189) \\
\hline \multirow{2}{*}{ 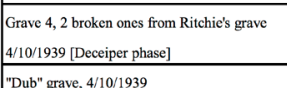 } & $\begin{array}{l}190 \\
191\end{array}$ & $\begin{array}{l}1355 \text { round vase, skctched } \\
\text { ordinary vase sketched (brushed incising) }\end{array}$ & $6-4$ jar,short rim & Karnack Brushed-Incised var. Fish Bayou & incised & plain: : Abraham 1 & sholl & sooting, probo, 1355 inked; Phillips photo 3937 (Hudd 190) \\
\hline & & & & & & & & \\
\hline \multicolumn{3}{|l|}{ Whaly grave with 5 piccess. 4/10/1939 } & (HSU Mussum Coll.) & & & & & \\
\hline \multirow{4}{*}{$\begin{array}{l}\text { Grave } 5 \text {, decp grave N } \\
\text { 4/22/1939 } \\
\text { [Social Hill phase] }\end{array}$} & 192 & 1350 cazula & $6-7$ bowl,carinated & Cook Engraved & engraved & Ezar 12 : plain & shell + grog & 1350 inked on base, Phillips photot 3930 (Hudd 192) \\
\hline & 193 & 1351 flat bowl & 6.5 bowl, simple & & plain & & sholl & 1351 inked on base, Phillips photo 3985 (Hudd 193) \\
\hline & 194 & large food jar & & & & & & \\
\hline & $\begin{array}{l}195 \\
196\end{array}$ & 1348 water bottle & $6-2$ bottle & Keno related & incised & plain : Bellarmine 9 (sim) & grog + shell & vertical neck, slight spool, Phillilis photo 3875 (Hudd 195) \\
\hline
\end{tabular}

Table 1. Reconstructed Grave Lots from Huddleston's Excavations at Trigg (3CL26) and Cook (3CL82). 


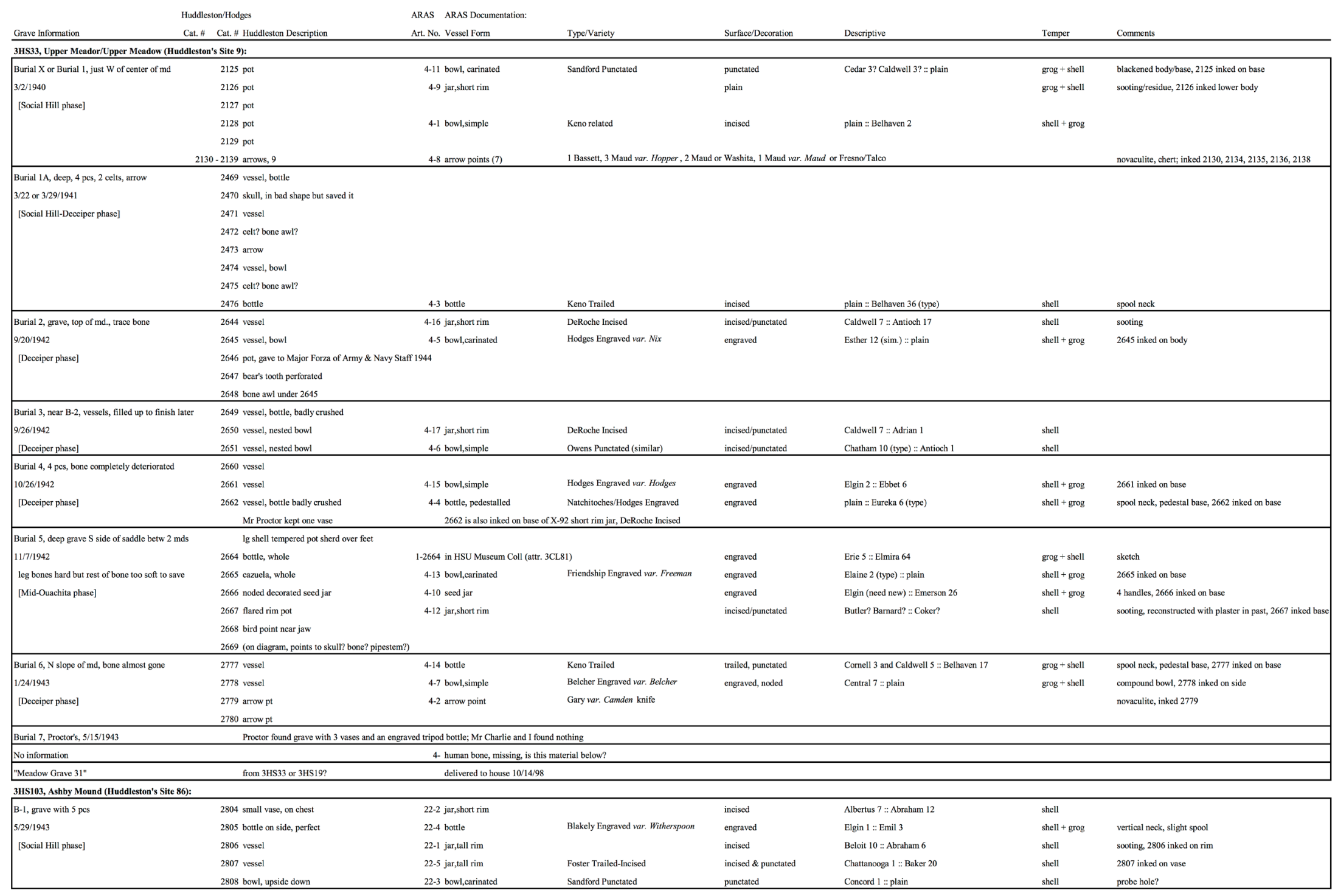

Table 2. Reconstructed Grave Lots from Huddleston's Excavations at Upper Meador (3HS33) and Ashby (3HS103). 
small mound about $20 \mathrm{ft}$ across and $1 \mathrm{ft}$ high." In May 1943, he dug one grave and recovered five vessels (77$1 / 22$, Table 2). Huddleston's notes indicate that " $\mathrm{Mr} \mathrm{C}$ and Mr Proctor" had visited the site previously (and there are two pots from Proctor attributed to this site in HSU Museum collections).

A low mound was noted at Upper Meador when it was first recorded into the Arkansas site file database in 1969. Additional collector digging at the site in the 1970s uncovered additional burials. The site was last visited by ARAS-HSU archeologists in 1980. No mound was mentioned on the site form at that time. Ashby is known only from Huddleston and Proctor notes and has not been visited by professional archeologists in the modern era.

\section{Lower Meador (3HS19)}

Huddleston's trips to Lower Meador or Middle Meadow (3HS19, his site 8) took place between 1940 and 1941, and he was often accompanied by Proctor, Richardson, "WS," and "Richard." In his typescript, he described the site as "a workshop, four burial mounds and at least three house mounds," but he seems to have focused on one mound. The first burial he dug (in March 1940) was a grave with two children and an adult, and he later dug additional child graves at the site. His notes on the 18 graves he dug are fairly detailed, and there is human bone in the JEC Hodges Collection linked with nine (eight with artifacts) of these graves. Burial 15, dug in June 1940, was "the deepest grave I ever opened, 5 feet to bottom of grave the strata was about as follows" (his sketch indicates 24 inches of "kitchen midden" over 24 inches of "midden and clay mixed" over a 12-inch stratum of "mingled[?] clay" containing the burial, above undisturbed clay). In his typescript, Huddleston recognized three types of burials at the site:

There were several very shallow ones on top of the mound. The pottery in these is typically marker type Caddo, very hard and polished, with some examples of the finest engraving we have ever taken out. In the flats, just south of the mound and mostly in midden, was a layer of graves about 18 to 24 inches deep, of about the same type. ... Nearly all of the pottery was shell tempered. ... A third level of burials was much deeper and just within the original soil. These graves were in the flat area just south of the mound and from four to six feet deep. All these graves were rich in pottery, one or two having as many as eleven pieces in them. The pottery was different in decoration and tempering. Most of it being sand or grit temper [Huddleston notes, on file ARAS-HSU].

A total of 49 vessels, 22 shell beads, four arrow points, three bone points, one bone awl, one celt, one mussel shell, one animal bone, and one pipe in the JEC Hodges Collection have been linked with 16 Huddleston graves from Lower Meador (77-1/2, Table 3), There are also seven vessels from Hodges's excavations at this site.

By the time Lower Meador was recorded into the Arkansas site file database in 1965, the landowner had bulldozed a mound and leveled the surface of the site. There was additional amateur digging that uncovered human remains at the site in 1972. A visit to the site at that time by an ARAS-HSU archeologist confirmed the location of 3HS19 and several nearby sites (3HS38, 3HS56). Collector digging uncovered additional graves with Caddo period materials at 3HS38 (see McKinnon 2015:123-124) and a large mound has been noted at 3HS56, raising the possibility that Huddleston included this larger site cluster in his description of multiple mounds at Lower Meadow.

\section{Stanford (3CL81), Richardson (3CL83), and Rorie} (3CL23)

Huddleston's work at the Stanford Farm or Stanford Place (3CL81, his site 7) took place between May 1938 and February 1943. The graves he dug there were in a mound "about $100 \mathrm{ft}$ across and round ... the highest elevation was only about 8 to $6 \mathrm{ft}$ above the surrounding ground." On several of his visits he mentions graves dug by Mr. Proctor and Mr. Richardson (40 vessels in HSU Museum collections are attributed to the Stanford site). Huddleston's draft manuscript mentions the large mound with numerous burials as well as "a small house mound and a midden site, covering about three-fourths acre." 
Based on his notes, Huddleston dug 18 graves at Stanford; 29 vessels from 14 of these graves have been identified in the JEC Hodges Collection (77-1/5, Table 4), along with two vessels in HSU Museum collections and one vessel "from Stanford mound." There is human bone in this collection, which could be from Huddleston's Burial 3 (it is labeled 7-3), or from Burial 21 (from which he kept the skull).

Huddleston described the Richardson Farm or Richardson Place (3CL83, his site 89) as "a small mound perhaps 18 " high and about 75 ' in circumference. There is deep midden on the east side and midden refuse is scattered over a considerable area." Huddleston dug at least eight graves here between November 1943 and March 1944. He was accompanied by "Mr. Charlie," Richardson, and "Mr. Proctor" (Robert Proctor), and mentioned that "Dr. Horton and his crew" (Philip Horton) were there at some point as well (HSU Museum collections include 18 vessels from 3CL83 collected by Horton, Proctor, and O. Whaley). Huddleston noted that Grave 10 was isolated from the main cluster of 16 (17?) graves dug by the group. Huddleston's record from this site include some vessel catalog cards and notes, providing descriptions of the graves he dug as well as some mention of those dug by the others.

There are 15 vessels and two earspools in the JEC Hodges Collection that can be linked to six Huddleston grave lots from Richardson (77-1/3, Table 4; one other vessel is from unknown context). Four of Huddleston's graves (three with artifacts) have human bone in the JEC Hodges Collection, and these have been transferred to ARAS-CO for NAGPRA documentation.

Huddleston's excavations at "a small mound on the Paul Rorie place" (3CL23, his site 6) took place between 1937 and 1944. He noted at his first visit in November 1937 that the "mound had been almost totally wrecked by other pot hunters." On his last visit in February 1944, he mentioned accompanying Proctor and Richardson (HSU Museum collections includes 41 vessels attributed to Proctor's excavations at Rorie). Huddleston's typescript describes "three mounds, two small and one large one. Across a small branch to the west is a large village site. Work shop refuse and sherds cover two or three acres."
From Rorie, Huddleston described 16 graves (plus another dug by "Mr Charlie"). A total of 23 vessels can be linked with 11 of these graves (77-1/8, Table 4; context is unclear for another six vessels). Most of these grave lots are incomplete.

The Arkansas site file database for Stanford (3CL81) includes notes on collector digging in the 1970s. A visit by ARAS-HSU archeologists in 1978 confirmed its location and added a Woodland period component to the record based on a surface collection. Richardson (3CL83) is recorded in the Arkansas site file database with an imprecise location and has not been visited by professional archeologists in the modern era. In 1969, ARAS-HSU archeologists visited Rorie (3CL23) and noted that the mounds described by Huddleston had been leveled. An extensive surface scatter (ca. 500 x $300 \mathrm{~m}$ ) remained with diagnostic artifacts indicating materials from the Late Archaic, Woodland, and Caddo periods. In 2002, I and a team of volunteers recorded patches of burned soil, post stains, midden debris, and human remains following land leveling at Rorie (Trubitt 2003). Some of these features could indicate the locations of the two smaller mounds.

\section{Vessel Analysis and Grave Lot Seriation}

The ceramic vessels from these eight sites have been documented using a protocol first developed by Ann Early for use on HSU Museum vessel collections and updated by Early and by Leslie Walker (2014). At the ARAS-HSU research station lab, we recorded attributes of form and shape, paste and temper, measurements, decorative treatment, evidence of use, as well as provenience (see Appendix).

A total of 167 vessels has been documented from the eight sites in this study. Of these, 37 (22 percent) are bottles, 63 (38 percent) are bowls (carinated bowls, simple bowls, and beakers), 58 (35 percent) are jars (both tall rim and short rim jars), and 9 (5 percent) are seed jars. Bottles and seed jars have small orifice diameters that would have restricted access to contents, while bowls and jars have larger orifice diameters allowing unrestricted access to vessel contents. All but two of the jars and bowls have orifice diameters less than $27 \mathrm{~cm}$ (Figure 2). The larger size category found in domestic contexts (e.g., in 


\begin{tabular}{|c|c|c|c|c|c|c|c|}
\hline Grave Inormation & Huddleston/Hodges & \multicolumn{2}{|l|}{ ARAS ARAS Documentation: } & Surface/Decoration & Dessripitive & Temper & \multirow{2}{*}{ Comments } \\
\hline \multicolumn{7}{|c|}{ 3HS19, Lower MeadorfLower Meadow (Huddleston's site 8): } & \\
\hline \multirow{13}{*}{$\begin{array}{l}\text { Burial I, multiple (2 children, I adult), } 9 \text { pots } \\
\text { 3/23 o o 3 3/24/1940 } \\
{\left[\begin{array}{l}\text { Deceiper phase] } \\
\text { Associated }\end{array}\right.}\end{array}$} & 2165 vessel with child & 2-18 bottle & Hodges Engraved var. Hodges & engraved & plain:: Ebbet 14 & shell + grog & short spood neck; ; roboce holc; 2165 inked bases, red pigment \\
\hline & 2166 beads, with child & & & & & & \\
\hline & 2167 vessel, with adult & 2-31 botle & Bailey Engraved & engraved & plain :: Eugene 3 & shell & bubbus spool neck, pedestal base; 2167 inked lower body \\
\hline & 2168 vessel, with adult & 2-19 jar, short rim & & incised & plain : : Berry 3 or 11 (sim.) & shell & sooting; 2168 inked on base \\
\hline & 2169 vessel, with adult & $2-5 \mathrm{jar}$, short rim & Ken relatad & incised & plain : : Belhaven 2 & sholl & 2169 inked near base \\
\hline & 2170 vessel, with child & & & & & & \\
\hline & 2171 skull, child & & & & & & \\
\hline & 2172 vessel, with child & 2.33 bowl, simple & & plain & & grog & minibowi; red chre on interior; 2172 inked \\
\hline & 2173 vessel, with child & 2-57 jar, short rim & & plain & & shell & sooting; 2177 inkcd on base \\
\hline & 2174 vessel, with child & $2-56$ jar, shor rim & Keno/Wallace? & incised & plain : : Buffalolo 1 (sim.) & shell & 2174 inked on base \\
\hline & 2175 unid on diagram, with child & 2-30 human, $1 \mathrm{pc}$ & & & & & \\
\hline & 2176 beads, with child & $2-28$ shell beads, 22 & & & & & \\
\hline & 2177 vessel, with child & 2-29 jar, short rim & & incised & Austin? ?: Buffalo 12 (sim.) & shell & sooting; 2177 inked on body \\
\hline \multirow{4}{*}{$\begin{array}{l}\text { Burial 2, child grave with bottle, skull too sof to save } \\
\text { 3/2441/1940 } \\
\text { [Deceiper phase] }\end{array}$} & 2178 vessel & 2.6 bowl,simple & 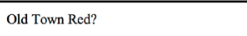 & red slipped & $G: G$ & shell & 2 small lugs at lip \\
\hline & 2179 vessel & 2-23 bowl,carinated & Hodges Engraved var. Nix? & engraved & Esther 4 (sim.): : plain & shell + grog & reconstructed with plaster in past \\
\hline & 2180 vessel & 2-11 jar, short rim & & plain & & & sooting; 2180 inked on base \\
\hline & 2181 vessel (cat. card) & 2-17 bottle & Hodges Engraved var. Hodges & engraved & plain :: Ebbet 14 & grog + sholl & short carafe/sppol neck; red pigment in engraving \\
\hline \multirow{4}{*}{$\begin{array}{l}\text { Burial 3, adult grave, skull soft } \\
\text { 3/24/1940 } \\
\text { [Deceiper phase] }\end{array}$} & 2182 perfect bottle & $2-35$ bottle & Hodges Engraved var. Hodges & engraved & plain: : Ebbet 5 (sim.) & shell + grog & bullows/spool neck \\
\hline & 2183 vessel & 2-12 bowl,carinated & Hodges Engraved var. Nix & engraved & Esther 12 (type) : plain & grog & \\
\hline & 2184 vessel & 2-50 jar,short rim & Mound Tract Inc./Brush. (Cowhide St.) & dentate stamped incisedbrushed & d Ferris 1 : Drury 1 & sholl & \\
\hline & 2185 sherd from large pot & $2-34 \mathrm{jar}$ (sherd) & Karnack Brushed-Incised var. Fish Bayou & brsshedtrailed & Dana 4 : : Abraham 1 & shell & rimsherd with sooting, 2185 inked on exterior \\
\hline \multirow{11}{*}{$\begin{array}{l}\text { Burial 4, adult grave, } 2 \text { pots, skull, } 7 \text { arrowpoints } \\
\text { 3/266,1940 } \\
\text { [Sociel Hill-Decciper phasec] } \\
\text { Associated }\end{array}$} & 2186 jar at feet, small animal bones \& muscle shell: & $2-52 \mathrm{jar}$, tall rim & Foster Trailed-ncised & punctated/incised/appliqued & Carbon 1 : : Baker 3 & shell & sootingressidue; 2186 inked on base \\
\hline & 2187 Giendora botlle & $2-4$ botle & Keno Trailed var. Reed Hill & inciscd & plain : : Bchlaven 31 & shell + grog & spool ncck, pedstal basc; 2187 inked on lower body \\
\hline & 2188 fint point & 2.7 arrow point & Gary var. Canden & & & & novaculite, inked 2188 \\
\hline & 2189 fint point & 2.8 arrow point & Womble or Scallorn? & & & & novaculite, inked 2189 \\
\hline & 2190 fint point & 2.9 arrow point & Maud var. Maud & & & & novaculite, inked 2190 \\
\hline & 2191 finit point & 2-10 arrow point & Bassett & & & & novaculite, inked 2191 \\
\hline & 2192 bone point & $2 \cdot 2$ nonhuman modificd & & & & & inked 2192 \\
\hline & 2193 bone point & 2-3 nonhuman modified & & & & & inked 2193 \\
\hline & 2194 bone point & $\mathrm{X}$ nonhuman modified & & & & & inked 2194 \\
\hline & 2195 vessel fragnnent bonce? & $\mathrm{X}$ nonhuman urmodificd & & & & & inked 2195 \\
\hline & 2196 skull & $2-65$ human, 2 pes & & & & & \\
\hline \multirow{5}{*}{ 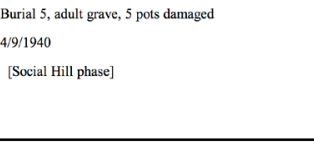 } & 2197 vessel & 2.47 bowl,simple & & plain & & shell & \\
\hline & 2198 vessel & $2-41$ jar,short rim & Emory Punctated-Incised? & incised/punctated & Caldwell 9 (sim.): Belmont 8 (yyee) & shell & probe hole \\
\hline & 2199 vessel & $2-48$ jar,tall rim & Foster Trailed-lncised variant? & brushedininisedappliqued & Defiance 1 (type) : Belmont 6 (type) & shell & \\
\hline & 2200 vessel & $2-45$ jar,tall rim & Foster Trailed-ncised variant? & incisedappliqued & Brevard 2 (sim.): : Bellarmine 7 (type) & shell & \\
\hline & 2201 vessel & 2-32 jar,tall rim & Foster/Pease? & incised & Alfred 2 : Antioch 18 (sim.) & shell & 2201 inked lower body \\
\hline Burial 6 , child grave, shallow, 4/13/1940 & 2202 arrow at head & & & & & & \\
\hline \multirow{6}{*}{$\begin{array}{l}\text { Burial 7, adult grave, so } \\
4 / 13 / 1940 \\
\text { [Social Hill phase] } \\
\text { Associated }\end{array}$} & 2203 vessel & & & & & & \\
\hline & 2204 vessel & $2-44$ jar, tall rim & Foster/Caney? & incised/punctated & Alfred 6 : Caney 5 & sholl & sootingressiduc: 2204 inked on body \\
\hline & 2205 vessel & & & & & & \\
\hline & 2206 vessel & 2.-53 bowl, simple \& 1 unmodif stell & & plain & & shell & 2206 inked near base \\
\hline & 2207 bonc awl \& large muscle shell under pot 2206 & X nonhuman modificd & & & & & Huddlession note: bonc awl and shell found under pot 2206 \\
\hline & 2208 skull in fair condition & 2.68 human, 2ppes & & jncised/nunctoted & & & \\
\hline \multirow{4}{*}{$\begin{array}{l}\text { Burial 8, adult grave, deep, } 5 \text { ' in } \\
\text { 4/20/1940 } \\
\text { [carly Mid-Ouachita phasc] } \\
\text { Associated } \\
\end{array}$} & 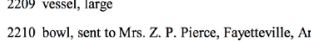 & $\begin{array}{l}2-43 \text { jar, tall rim } \\
\text { rkk, } 1941\end{array}$ & Military Road Incised & incised/punctated & Bethel 1::Bard 27 (sim.) & grog & sooting; 2209 inked near base \\
\hline & 2211 vessel & $2-40$ jar, tall rim & Military Road Incised & inciscd/punctated & Beloit 9 : Bard 1 & grog & sooting, reconstructed with plaster; 2211 inked near basc \\
\hline & 2212 Caddo pipe under head & 2.54 loop pipe & & & & & inked 2212 \\
\hline & 2213 indicated skull or mandible on diagram & 2.76 human, 6 pes & & & & & \\
\hline
\end{tabular}

Table 3. Reconstructed Grave Lots from Huddleston's Excavations at Lower Meador (3HS19). 


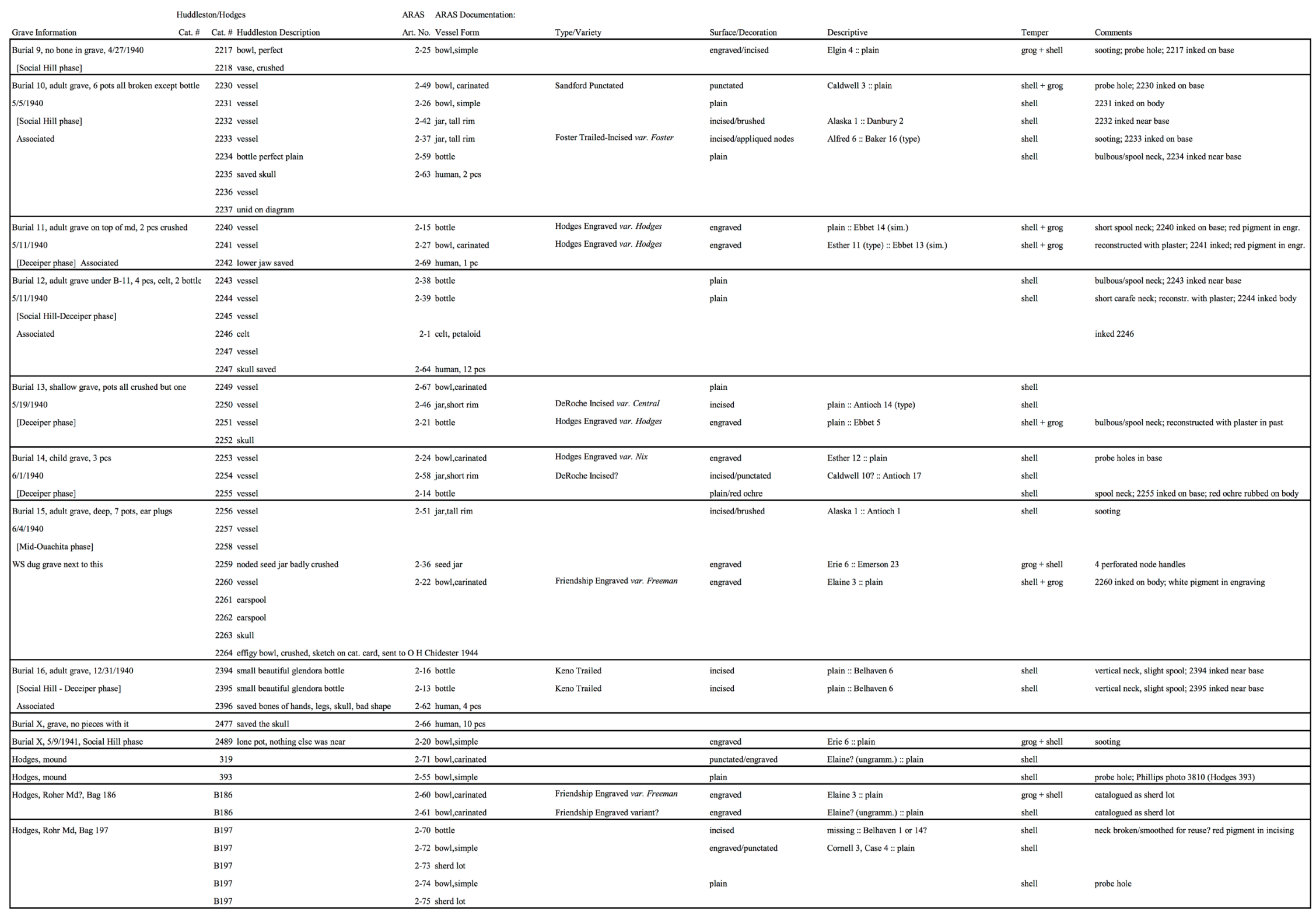




\begin{tabular}{|c|c|c|c|c|c|c|c|c|}
\hline Grave Information & $\begin{array}{l}\text { Huddles } \\
\text { Cat.\# }\end{array}$ & $\begin{array}{l}\text { on/Hodges } \\
\text { Cat. \# Huddleston Description }\end{array}$ & $\begin{array}{l}\text { ARAS ARAS Documentation: } \\
\text { Art. No. Vessel Form }\end{array}$ & Type/Variety & Surface/Decoration & Descriptive & Temper & Comments \\
\hline \multicolumn{9}{|c|}{ 3CL81, Stanford (Huddleston's Site 7): } \\
\hline \multirow{2}{*}{$\begin{array}{l}\text { Burial 1, in mound, trace bone, } 3 \text { pots } \\
\text { 5/14/1938 }\end{array}$} & 43 & 1303 bowl, whole, rim projections (skecth) & 1-1303 car. bowl, in HSU Museum coll & Garland Engraved & engraved & Elgin 2 : : Elmira 32 & none (mica) & rattle nodes at rim; Phillips photo 3890 (Hudd 43) \\
\hline & 44 & 1340 bowl, broken, handle at rim (sketch) & 14-20 effigy bowl & & appliqued handles & & grog + shell & 1340 inked; Phil 3904 (Hudd 44), Freeman [no, Stanford] \\
\hline \multirow{2}{*}{\begin{tabular}{|l|} 
[Mid-Ouachita phase] \\
Burial 2?, 5/4 4/1938, grave to N of B-1 \\
\end{tabular}} & 45 & bowl, broken, 4 tips on rim (skecth) & & & & & & \\
\hline & & small pot of muscles shell all broken up & & & & & & \\
\hline \multirow{4}{*}{$\begin{array}{l}\text { Burial 3, skeleton lying E-W with } 4 \text { pots } \\
\text { 5/20/1938 } \\
\text { [Mid-Ouachita phase] }\end{array}$} & 46 & 1238 large water bottle, broken & $5-18$ bottle & & plain & & shell & verrical neck, sl. spool; 1238 inked; Phil 3986 (Hudd 46) \\
\hline & 47 & double human effigy water bottle (sketch) & not located in HSU Mussum coll & sim. to Bailey Engraved & & & & Phillips photo: 3895 (Hudd 477hoie says at HSTC \\
\hline & 48 & flared bowl & & & & & & \\
\hline & 49 & small water bottle with neck gone & & & & & & \\
\hline \multirow{2}{*}{\begin{tabular}{|l|} 
Burial $5,5 / 2411938$, grave w war, trace bone \\
Burial $6,5 / 2411938$, Proctor (2 burial levelals) \\
\end{tabular}} & 50 & only one small jar and trace of bone & & & & & & \\
\hline & & second one with 5 pots, 2 chisels, double wat & er bottle & & & & & \\
\hline \begin{tabular}{|l|} 
Burial $7,5 / 28 / 1938,1$ pot, no trace bone \\
\end{tabular} & 51 & 1307 water bottle (sketch) & 5-31 bottle & Keno Traildd var. Red Hill & incised & Bates 5 : : Belhaven 5 (sim.) & shell & 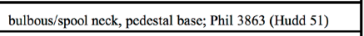 \\
\hline \multirow{2}{*}{$\begin{array}{l}\text { Burial 8, 5/28/1938, under B-7, } 2 \text { pots } \\
\text { [Mid-Ouachita?? phase] }\end{array}$} & 52 & 1436 Rim bowl, white pigment (sketch) & 5-17 bowl,carinated & $\begin{array}{ll}\text { Friendship Engraved var. Trigg? } \\
\end{array}$ & engraved & Erie2 2: Emporia 2 : plain & shell & 1436 inked; Phillips photot0 9903 (Hudd 52) \\
\hline & 53 & badly crushod, double built bowl, gave to WS & repaired & & & & & \\
\hline Burial 10, 5/28/1938, grave w crushed jar & & food jar of muscle shell construction, not save & & & & & & \\
\hline \multirow{6}{*}{$\begin{array}{l}\text { Burial 11, grave w/ } 5 \text { pots, one on chest } \\
5 / 30 / 1938 \\
\text { [Social Hill phase] }\end{array}$} & 54 & 1292 & 5-30 jar,tall rim & $\begin{array}{l}\text { Foster Trailed-lncised var Fosster } \\
\end{array}$ & incised applique & Alfred 6 : Baker 22 & shell & Phillips photo: 3911 (Hudd 54, handwr. 1294 [no 1292]) \\
\hline & 55 & 1308 Flat rim bowl, sketch & 5-15 bowl,carinated & Sandford Punctated & punctated & Caldwell 3 : : plain & shell & Phillips photo 3878 (Hudd 55) \\
\hline & 56 & 1289 jar, sketch & 5-10 jar,shor rim & & incised punctated & Concord 3: Buffalal 14 & shell & sootingrresidue; probe hole; Phillips photo 3856 (Hudd 56) \\
\hline & 57 & 1239 water bottle, skctch & 5-2 bottle & Keno Traild & incised & plain : : Belhaven 1, 18 (sim.) & grog + git & bulbous/spool neck, pedcstal; Phillips ph 3896 (Hudd 57) \\
\hline & 58 & 1298 jar, sketch & $5-11$ jar,tall rim & Foster Trailed-Incised var Fosser & incised applique & Alfred $2:$ : Baker 4 & shell & sooting: 1298 inked; Phillips photo 3852 (Hudd 58) \\
\hline & & & 5.25 bow1,simple & Hardman Engraved (similar) & incised (fine line) & Bates 2? : : plain & shell & no inked \#; 1977 cat has $5-25$ in B11 but unclear why \\
\hline \multirow{5}{*}{$\begin{array}{l}\text { Burial 12, grave adj io B-11, } 4 \text { pois } \\
\text { 5/30-5/31/1938 } \\
\text { skull in fair shape, gave to C. Richardson } \\
\text { [Mid-Duachita phase] }\end{array}$} & 59 & 1290 sed jar & $5-20$ seed jar & & plain & & shell & 1290 inked on lower body; Phillips photo 3905 (Hudd 59) \\
\hline & 60 & 1287 food jar muscle shell & 5.32 jar,tall rim & Military Road Incised & incised/punctated & Beloit 1: Bard 30 (type) & grog+ shell & sooted, 1287 inked; Phill lips photo 3913 (Hudd 60) \\
\hline & 61 & small jar samec composition & & & & & & \\
\hline & 62 & Caddo pipe, black pottery dipped in red clay & & & & & & \\
\hline & 63 & $\begin{array}{l}1244 \text { rim bowl crushed } \\
\text { flint scraper }\end{array}$ & 5-12 bowl,carinated & Friendship Engraved var. Fremaman & engraved & Elaine 3 : plain & shell + grog + grit & Phillips photo 3984 (Hudd 63) \\
\hline \multicolumn{2}{|l|}{\begin{tabular}{|l|l} 
Burial 13, 5/31/1938, Richardson \\
\end{tabular}} & Richardson, bottle and small rim bowl & & & & & & \\
\hline \multicolumn{2}{|l|}{ Burial 14, 64:1938, Richardson } & 2 bowis with handles, large food jar, decp mi & xing bowl & & & & & \\
\hline \multicolumn{2}{|l|}{ Burial 15, 64:-67/1938, Proctor } & 4 jars and 3 more wrecked pots & & & & & & \\
\hline \multirow{6}{*}{\begin{tabular}{|l} 
Burial 16, 6/5/1938, 1 crushed pot, no bone \\
Burial 17, 5 pots, trace bone \\
6/11/1938 \\
about $6 \mathrm{Nof}$ Mr R's Grave 13 \\
[Social Hill phase]
\end{tabular}} & 217 & 14021 crushed pot, sketch (part of B-19 to E?) & 5-9 bowl,simple & & engraved & plain: : Evanssille 4 & shell + bone & probed; 1402 inked; Phillips photo 3907 (Hudd 217) \\
\hline & 64 & 1245 small food jar & 5-14 jar,tall rim & Foster Trailed-Incised & incised & Alfred $2:$ : Bellarmine 13 (type) & shell & Phillips photo 3862 (Hudd 64) \\
\hline & 65 & 1284 double water bottle, sketch & 5-16 bottle & Adair Belcher Engraved & engraved & Elgin 26 : Edith 2 (sim.) & shell + grit & verical neck, slieht spool; Phillips photo 3915 (Hudd 65) \\
\hline & 66 & 1242 small flat rim bowl & 5.4 bowl,carinated & Cook Engraved & engraved & Eddy 3: plain & shell & 1242 inked on basc; Phillips photo 3902 (Hudd 66) \\
\hline & 67 & small flat rim bowl, red material, very soft & & & & & & \\
\hline & 68 & 1437 food jar & $5-6$ jar,tall trim & Caney Punctated variant? & brushedridge pinched & Dana 4 : Curry 4 (type) & shell & blackened int; ; Phililips phoro 3916 (Hudd 68) \\
\hline \multirow{8}{*}{$\begin{array}{l}\text { Burial 19, grave w } 8 \text { pots, some crushed } \\
\text { 6/1 1/1938 } \\
\text { skull too sof to to save } \\
\text { [Social Hill-Deccipor phase] }\end{array}$} & 69 & jar & & & & & & \\
\hline & 70 & bottle, wrecked & & & & & & \\
\hline & ${ }^{71}$ & jar & & & & & & \\
\hline & 72 & 1240 bowl & 5-19 jar,tall rim & Foster Trailed-Incised & incisedapplique & Agnes 1 : Bakcr 26 (sim) & shell & proboc holl; Phillips photo 3864 (Hudd 72) \\
\hline & 73 & bottle, wrecked & & & & & & \\
\hline & 74 & tall rim jar too rotton to save & & & & & & \\
\hline & 75 & double bottle one end crushed & no \# in HSU Museum coll as no prov & & & Evansville 4 & & Phil 3999 (Hudd 75)) HSU Mus No Prov photoo 714319 \\
\hline & 76 & botlle & & & & & & \\
\hline Burial 20, 11/12-11/13/1938, grave w 1 pot & 86 & food jar, skecth (vert lines on body) & & & & & & Phil 3997 (Hudd 80); notebook: grave 19 opened 11-13-38 \\
\hline \multirow{4}{*}{$\begin{array}{l}\text { Burial 21, lying E-W, } 4 \mathrm{pc} \\
11 / 26 \text { (2938 } \\
\text { [Mid-Ouachita phases] }\end{array}$} & 89 & food jar whole & & & & & & \\
\hline & 90 & 1285 small cooking jar, skecth & 5-24 jar,tall rim & Military Road hnised & incised + punctated & Bethel $1:$ :Bard 2 & grog + shell & blackened, 1285 inkd on rim; Phillips photo 3912 (Hudd 90) \\
\hline & 91 & casulla cushed & & Friendship Engraved var. Meedor & engeraved & Elwyn 3 : plain & & Phillips photo 3908 (Hudd 91) \\
\hline & 92 & $\begin{array}{l}\text { mixing bowl / serated, sketch } \\
\text { saved hhe skull in fair condition }\end{array}$ & & Hardman Engraved? & engravednotched lip & & & Phillips photo 3995 (Hudd 92) \\
\hline
\end{tabular}

Table 4. Reconstructed Grave Lots from Huddleston's Excavations at Stanford (3CL81), Richardson (3CL83), and Rorie (3CL23). 


\begin{tabular}{|c|c|c|c|c|c|c|c|c|}
\hline & Huddlest & onHodges & ARAS ARAS Documentation: & & & & & \\
\hline Grave Information & Cat. \# & Cat.\# Huddleston Description & Art. №. Yessel Form & Type/Variety & Surface/Decoration & Descriptive & Temper & Conments \\
\hline Burial 23, grave w 2 or 3 pots, no bone & 87 & 1293 casuela bowl badly crushed & & Friendship Engraved var: Medor & engraved & Elwyn 2:: plain & & Phillips photo 3909 (Hudd 87, handwritten 1293) \\
\hline $11 / 26 / 1938$ & 88 & 1283 casucla bowl badly crushed & 5-21 bowl,carinated & Friendship Engraved var: Meador & engraved & Elwyn 2 : plain & grog & 1283 inked near base; Phillips photo 3952 (Hudd 88) \\
\hline [early Mid-Ouachita phase] & & 1 food jar only partly there & & & & & & \\
\hline Burial 26, deep grave, 5 pots, no bone & 169 & 1314 water bottle perfect broken rim of neck & $5-8$ bottle & & plain & & shell & bulboussppool neck; Phillips photo 3880 (Hudd 169) \\
\hline 3/25/1939 & 170 & 1282 small vase & 5-7 jar,short rim & Karnack Brushed-Incised? & incised & Austin 1 : Abraham 3 (type) & shell + bone & Phillips photo 3884 (Hudd 170) \\
\hline \multirow[t]{3}{*}{ [Social Hill-Decciper phase] } & 171 & $171 \& 172$ cazuella inverted over a vase & & & & & & \\
\hline & 172 & the vase too near gone to save & & & & & & \\
\hline & 173 & 1392 perfect water bottle, trailed design (sketch) & $5-3$ bottle & Keno Trailed & incised & plain : Belhaven 1 & shell & bulbous/spool neck; Phillips photo 3892 (Hudd 173) \\
\hline Burial $26(277), 3$ pots incl turtle effigy & & 2397 & $5-5$ bottle & Blakely Engraved var. Blakely & engraved & plain : : Esop 3 & grog + shell & vertical neck, slight spool; 2397 inked near base \\
\hline 1/4/1940 or 1/4/1941 & & 2398 & & & & & & \\
\hline \multirow[t]{2}{*}{ [Mid-Ouachita-Social Hill phase] } & & 2399 effigy & $5-23$ effigy bowl & Means Engraved & engraved appliqued flangeshand & I Elgin 1 : : plain & shell & \\
\hline & & 2410 bowl or broken bottle? & 5-29 bowl,simple & & engraved & Eric??: Elmira? (sim.) & grog + grit & probe hole \\
\hline Burial $\mathrm{X}$, grave $\mathrm{w} / \mathrm{S}$ pots, no bone & & 2784 vessel & & & & & & \\
\hline $\begin{array}{l}\text { 2/28/1943 } \\
\text { [Social Hill-Deceiper phase] }\end{array}$ & & $\begin{array}{l}2785 \text { jar } \\
2786 \text { vessel }\end{array}$ & 5-28 jar, tall rim & Foster Trailed-Incised & incised & Agnes $1:$ : Baker 1 & shell & blackened interior \\
\hline [Social Hill-Deceiper phase] & & $\begin{array}{l}2786 \text { vessel } \\
2787 \mathrm{jar} \\
2788 \text { bottle }\end{array}$ & 5-13 jar,tall rim & & incised & Albertus 4 : Abraham 1 & shell & 2787 inked near base \\
\hline Huddleston, Stanford mound & 213 & 1241 vessel & 5-1 bowl,simple & Keno related & incised & plain: : Belhaven 2 & shell & sooting? Phillips photo 3889 (Hudd 213) \\
\hline No info (Huddleston 7-3= Stanford Burial 3?) & & 1404 & 5-26 human, 2 pcs & & & & & cranium inked 1404, 7-3-A in pencil; mandible 7-3-A pencil \\
\hline No info (Huddleston 7-3 = Stanford Burial 3?) & & 1405 & 5-27 human, 2 pcs & & & & & mandible inked 1405a, 7-3-B in pencil; cranium \\
\hline \multicolumn{9}{|l|}{ 3CL83, Richardson (Huddeston's site 89): } \\
\hline Grave 1 , no skull found, 3 pieces & & 2842 cazuela & & & & & & \\
\hline $11 / 201943$ & & 2843 bowl, erushed, sketch on cat. card & 3-18 jar,short rim & Cowhide/Foster? & dentate stamped incised appliqu & $\backsim$ Ferris 1 : : Baker 2 & shell & sooting \\
\hline \begin{tabular}{|l} 
[Social Hill-Deceiper phase] \\
\end{tabular} & & 2844 bottle broken, opening covered with sherd & 34 bottle (reused as seed jar) & Belcher Engraved var. Soda Lake & engraved & plain : Elizabeth 1 & grog + bone + shell & 1 bottle neck broken/smoothed for reuse as seed jar \\
\hline Grave 3,5 pots & & 2845 owl efingy seed jar, skectch on cat. card & 3-9 effigy seed jar & & appliqued nodes & Harvey: : plain & shell & probe hole, 2845 inked on base (Trubitt 2017) \\
\hline $11 / 20 / 943$ & & 2846 pot, sketched on cat. card & 3-10 jar, short rim & & incised & Barnard? or Bates 10 ? : Baker 18 & shell & sooting, 2846 inked near base \\
\hline [Social Hill phase] & & 2847 vase, skecthod on cat. card & 3-15 jar, tall rim & Foster Trailed-Incised & incised/punctatedapplique & Concord 10 or Chattanooga 7 (sim.) : : Baker 23 (sim.) & shell & sooting, 2847 inked near base \\
\hline \multirow{3}{*}{\multicolumn{2}{|c|}{ Associated }} & 2848 cazucla, bowl skectched on cat. card, Bur 2? & 3-17 bowl, carinated & Sandford Punctated & punctated & Caldwcll 6? : plain & shell & 2848 inked on base \\
\hline & & 2849 vessel on diegram & & & & & & \\
\hline & & 2850 human, on diagram & 3-21 human, $40 \mathrm{pcs}$ & & & & & \\
\hline \multirow{2}{*}{\multicolumn{2}{|c|}{ Grave 4, 1 pot, 11/20/1943 }} & 2851 point on diagram & & & & & & \\
\hline & & 2852 vessel on diagram & & & & & & \\
\hline Grave 6 , below others, 3 (4) pots & & 2853 vessel, sketch on card & 3-8 beaker or mini-jar & & punctated & plain :: Catawba 2? & shell & 2853 inked on base \\
\hline 11/20-21/1943 [Social Hill phase] & & 2854 Glendora bottle, sketch on card & & Keno Trailed & & & & \\
\hline \multirow{2}{*}{\multicolumn{2}{|c|}{ Associated }} & $\begin{array}{l}2855 \text { cazuela, punctated rim, sketch on card } \\
2859 \text { skull indicated on diagratm }\end{array}$ & 3-6 bowl, carinated & Sandford Punctated & punctated & Caldwell 3 or 6 : plain & shell + grog & 2855 inked on base \\
\hline & & $\begin{array}{l}2899 \text { skull indicated on diagram } \\
2860 \text { bone (note on diagram about healed fracture) } \\
\text { camella, given to Proctor }\end{array}$ & 3-20 human, $4 \mathrm{pcs}$ & & & & & \\
\hline \multirow{3}{*}{\begin{tabular}{|l} 
Grave 9,3 pots (Horton took skull) \\
11/21/1943 \\
[Mid-Ouachita phase]
\end{tabular}} & & 2856 bottle, sketch on card & 3-13 bottle & & plain & & shell & verical neck, slight spool \\
\hline & & 2857 vase, sketch on card & 3-14 jar,short rim & Foster/Pease? & incised & Alfred 2: Alpha 1 & shell & \\
\hline & & 2858 bowl, skeech on card & 3-3 beaker & & noctched lip & Cornell 10 : plain & shell & deep bowl or beaker, 2858 inked lower body \\
\hline \multirow{5}{*}{\multicolumn{2}{|c|}{$\begin{array}{l}\text { Grave 10, isolated grave, } 3 \text { pes (bottle), earspools } \\
\text { 11/25/1943 } \\
\text { [Deceiper phase] } \\
\text { Associated }\end{array}$}} & 2861 bottle, engraved (cat card, repaired 4/20/44) & 3-5 bottle & Hodges Engraved var. Hodges & engraved & plain: : Ebbet 6 (sim.) & grog + shell & bulbous/ppol neck, 2861 inked on base \\
\hline & & 2862 bowl, sketch on card & 3-16 bowl, carinated & Ripley Engraved? & engraved & Erin (necd new) : :plain & shell + grog & blackened int, 2862 inked on body ext. \\
\hline & & 2863 earspool, sketch on card & 3-1 earspool & & & & shell + grog & ceramic, inked 2863 \\
\hline & & 2864 earspool & 3-2 earspool & & & & shell + grog & ceramic, inked 2864 \\
\hline & & $\begin{array}{l}2865 \text { vase, sketch on card } \\
2895 \text { bone (diagram indicates femur) }\end{array}$ & $\begin{array}{l}\text { 3-11 jar, tall rim } \\
3-19 \text { human, } 3 \text { pcs }\end{array}$ & Foster Trailed Incised & incised/appliqued nodes & Andes 9 :: Baker 22 & sholl & sooting, 2865 inked on base \\
\hline
\end{tabular}




\begin{tabular}{|c|c|c|c|c|c|c|c|c|}
\hline Grave Information & $\begin{array}{l}\text { Huddes } \\
\text { Cat \# }\end{array}$ & $\begin{array}{l}\text { Fion Hodges } \\
\text { Cat. If Huddleston Descriphion }\end{array}$ & $\begin{array}{l}\text { ARAS ARAS Documentation: } \\
\text { Art. No. Vessel Form }\end{array}$ & Type/Variety & Surface/Decoration & Descriptive & Temper & Conments \\
\hline $\begin{array}{l}\text { Grave 15, deep, in clay, } 7 \text { pieces } \\
22 / 511944 \\
\text { [Mid-Ouachita phase }]\end{array}$ & & $\begin{array}{l}2866 \text { vessel } \\
2867 \text { cazuella, incised, skecth on card } \\
2888 \text { gave pot to Major Forra, Arriny \& Nayy Staff } 194 \\
2869 \text { vessel } \\
2870 \text { gave pot to Major Forza, Arrmy \& Nary Staff } 194 \\
281 \text { bottle, plain, skctch on card } \\
2872 \text { vessel }\end{array}$ & $\begin{array}{l}\text { 3-12 bow, carinated } \\
944 \\
944\end{array}$ & Friendship Engraved var. Meador & engraved & Elwyn 3 (type) : plain & grog + grit & blackened interior \\
\hline Grave $16 \& 17$, Procotor, double burial & & 7 pes ince double loop borle (1-340-RP), 2 canue & & & & & & \\
\hline Grave $16(1879), 3 / 111944$, no goods & & 2900 legs; skeleton too sof to save, skull position & $\mathrm{X}$ human, $79 \mathrm{pcs}$ & & & & & \\
\hline 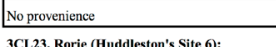 & & vessel & 3-22 bottle, plain (sherd lot) & & plain & & $\begin{array}{c}\text { grog + shall } \\
\text { f }\end{array}$ & unreconstructed shord lol, straight neck, sigight spool, botlle \\
\hline 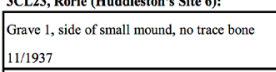 & $\begin{array}{l}35 \\
36 \\
\end{array}$ & $\begin{array}{l}\text { rim bowl, gave to Olin 4/11/38 } \\
\text { water bottle, red dlay, not put together yet 2/13/3/3 }\end{array}$ & & & & & & \\
\hline \begin{tabular}{|l|} 
Grave 2, same md as G-1, 3 ' deep, no bone \\
12/222/1938[1937?] [Mid-Ouachita phase]
\end{tabular} & 37 & $\begin{array}{l}1203 \text { rim bowl engraved desigg } \\
\text { other pots too baally wrecked to save }\end{array}$ & 8-11 bowl,carinated & $\begin{array}{l}\text { Friendship Engraved var. Freeman } \\
\end{array}$ & engraved & Elaine 1: plain & grog + shell & probe hole? inked 1203; Phillips photo 3956 (Hudd 37) \\
\hline 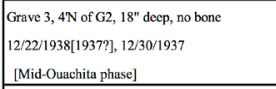 & $\begin{array}{l}38 \\
39\end{array}$ & $\begin{array}{l}1207 \text { cffigy shaped likc a frog } \\
\text { rim bowl lazy S engraved desgin } \\
\text { small food jar } \\
\end{array}$ & $\begin{array}{l}\text { 8-1 cffigy sced jar, quadruped } \\
8 \text { 8.25 bowl,carinated }\end{array}$ & Friendship Engraved var. Meador & $\begin{array}{l}\text { inciscd/modcldd } \\
\text { engraved }\end{array}$ & Elwyn 10 : : plain & $\begin{array}{l}\text { grog } \\
\text { grog }+ \text { shell }\end{array}$ & 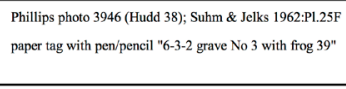 \\
\hline \begin{tabular}{|l} 
Grave 4, E slope mo, 24"doep, no trace bone \\
3/19/1938 \\
[Early Mid-Ouachita phasec]
\end{tabular} & $\begin{array}{l}40 \\
41\end{array}$ & $\begin{array}{l}1220 \text { largest rim bowl, engraved, over } 41 \\
1229 \text { large vase like jar } \\
\text { anohther rim bowl } \\
\text { anohher jar } \\
\end{array}$ & $\begin{array}{l}8-22 \text { bowl,simple } \\
8-23 \text { jar,tall rim }\end{array}$ & $\begin{array}{l}\text { Friendship Engraved var. Meedior } \\
\text { Militiary Road nncised }\end{array}$ & $\begin{array}{l}\text { eneraved } \\
\text { incised \& punctated }\end{array}$ & $\begin{array}{l}\text { Elwyn } 15 \text { (sim.) : : plain } \\
\text { Bethel } 1 \text { : Bard 15 }\end{array}$ & $\begin{array}{l}\text { grog } \\
\text { grog }\end{array}$ & $\begin{array}{l}1220 \text { inked on bass; Phillips phot } 3955 \text { (Hudd 40) } \\
\text { sooting, } 1229 \text { inked; Phillips photo } 3938 \text { (Hudd 41) }\end{array}$ \\
\hline $\begin{array}{l}\text { Grave } 5, \text { NE slope of md, no trace of bone } \\
3 / 19 / 1938 \\
\text { [early Mid-Ouachita phase] }\end{array}$ & 42 & $\begin{array}{l}1236 \text { large mouth water bortle ecthed dosign } \\
\text { vase like jar } \\
\text { vase like jar } \\
\text { rim bowl } \\
\text { rim bowl } \\
\end{array}$ & 8.3 botle & Maddox Engraved? & engraved & plain: : Eric 10, Eric 28 (type) & grog & insloping neck; refired vessel?; Phil 3861 (Hudd 42) \\
\hline \begin{tabular}{|l}
$\begin{array}{l}\text { Grave 6, field next to road } \mathrm{N} \text { of Rorie } m \text { md } \\
\text { 12/22/1938 } \\
{[\text { [Social Hill-Deceiper phase] }}\end{array}$ \\
\end{tabular} & $\begin{array}{l}103 \\
104\end{array}$ & $\begin{array}{l}1205 \text { water bottle, trailed design skecthod } \\
\text { small cazuela bowl } \\
\text { skull crushed, saved lower jaw } \\
\end{array}$ & $8-28$ botlle & Keno Trailed var: Red Hill & incised & plain : : Belhaven 31 & shell & bulbousspool neck, pecdstall; Phil 3917 (Hudd 103, 1205) \\
\hline $\begin{array}{l}\text { Grave 7, Eslope of Roric md, skull decaysd } \\
1 / 1 / 41 / 1938 \text { (1939) } \\
\text { [Mid-Ouachita phase] }\end{array}$ & $\begin{array}{l}114 \\
115 \\
116 \\
117 \\
118 \\
119\end{array}$ & $\begin{array}{l}1225 \text { secd jar in perfect condition } \\
1227 \text { cazuela invered o ver } 116 \\
\text { food jar } \\
\text { large sed jar badlly broken } \\
1211 \text { cazuela on edge, skecth in noles } \\
\text { water botlle perfect, skecth } \\
\end{array}$ & $\begin{array}{l}\text { 8-10 sced jar } \\
\text { 8-15 bowl, carinated }\end{array}$ & Friendship Engraved var. Freman & $\begin{array}{l}\text { plain } \\
\text { engraved }\end{array}$ & Elaine 13 (type) : : plain & $\begin{array}{l}\text { grog + shcll } \\
\text { shell }+ \text { grog }\end{array}$ & $\begin{array}{l}\text { probec holes, } 1225 \text { inkd, Prillips photo } 3935 \text { (Hudd 114) } \\
1227 \text { inked on bass; Phillips photo } 3958 \text { (Hudd 115) } \\
1211 \text { inked on base, Phillips photo } 3988 \text { (Hudd 118) }\end{array}$ \\
\hline \begin{tabular}{|l} 
Grave 8, on E, 30" deep, no trace skeleton \\
1/1/4/1938 (1939), , 1/21/1939 \\
[early Mid-Ouachita phase]
\end{tabular} & $\begin{array}{l}120 \\
121 \\
122 \\
123 \\
124 \\
125\end{array}$ & 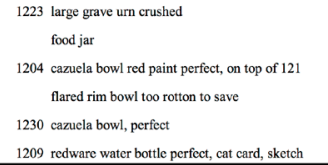 & $\begin{array}{l}8-16 \text { jar,tall rim } \\
8.26 \text { jar,all tim } \\
8.4 \text { bowl,simple } \\
8.5 \text { bowl,simple } \\
8.2 \text { bottle }\end{array}$ & $\begin{array}{l}\text { Milliary Road hnoised } \\
\text { Military Road Incised } \\
\text { Hempstead Engraved? } \\
\text { Hempstead Engraved } \\
\text { Crocket Curvilinear Incised (similar) }\end{array}$ & $\begin{array}{l}\text { incised \& punctated } \\
\text { incised \& punctated } \\
\text { engraved } \\
\text { engraved } \\
\text { engraved }\end{array}$ & 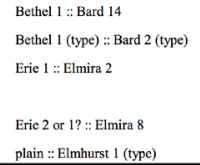 & $\begin{array}{l}\text { grog } \\
\text { grog } \\
\text { grog } \\
\text { grog } \\
\text { grog tshell }\end{array}$ & 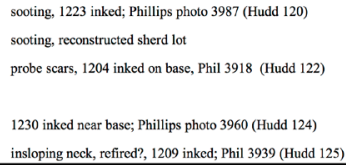 \\
\hline $\begin{array}{l}\text { Grave 9, about } 8 \text { ' S of G-8, no bone, } 5 \text { pots } \\
\text { 1/21/1939 } \\
\text { [early Mid-Ouachita phase] }\end{array}$ & $\begin{array}{l}126 \\
127 \\
128 \\
129 \\
130 \\
\end{array}$ & $\begin{array}{l}1219 \text { round bowl inverted, skecthod } \\
\text { crushed food jar } \\
1228 \text { crushed jar } \\
1389 \text { bowl, savtooth rim } \\
1126 \text { crnshed seed jar, reparired } \\
\end{array}$ & $\begin{array}{l}\text { 8-14 jar, short rim } \\
\text { 8-17 bowl,simple }\end{array}$ & Hempstead Engraved? & $\begin{array}{l}\text { incised \& punctated } \\
\text { engravecd/cxised }\end{array}$ & $\begin{array}{l}\text { Beloit 6: Abrahan } 6 \\
\text { Corncll 9, Elgin 1: : plain }\end{array}$ & grog + shell & $\begin{array}{l}\text { sooting, Phillips photo } 3872 \text { (Hudd 128) } \\
1389 \text { inked; Phillips photo 3954 (Hudd 129) }\end{array}$ \\
\hline \begin{tabular}{|l|} 
Grave 10, shallow grave in md, 2/11/1939 \\
\end{tabular} & 149 & celt in perfect condition (pols were smashed) & & & & & & \\
\hline \begin{tabular}{|l|} 
Grave 11, shallow grave in md, 2/11/1/1939 is \\
Roric Mound
\end{tabular} & $\frac{151 ?}{212}$ & $\begin{array}{l}1208 \text { perfect secd jar on oly } 8 \text { " under the surface } \\
1233 \text { vessel }\end{array}$ & $\begin{array}{l}8-19 \text { sced jar } \\
8-21 \text { jartall tim }\end{array}$ & Military Road Incised variant? & $\begin{array}{l}\text { plain } \\
\text { incised/punctatedapplique }\end{array}$ & Bethel 1: Bard 10, 40 (sim.) & $\begin{array}{l}\text { sholl + grog } \\
\text { sholl }\end{array}$ & 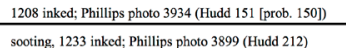 \\
\hline Rorie Mound & 214 & 1224 vessel & $8-13$ beaker & Hempstead Engraved & engraved & Erie 13: Eccles 1 & shell + grog & perforated lugs; 1224 inked; Phil 1859 (Hudd 214) \\
\hline
\end{tabular}

Table 4 continued. Reconstructed Grave Lots from Huddleston's Excavations at Stanford (3CL81), Richardson (3CL83), and Rorie (3CL23). 
Huddleston/Hodges $\quad$ ARAS ARAS Documentation.

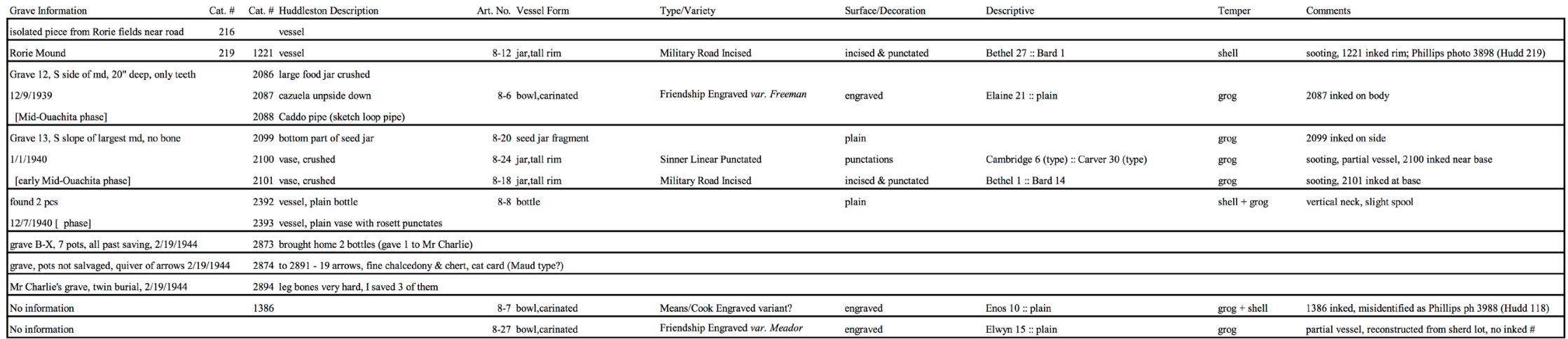


the Red River valley [Kelley 1997:39]) is rare in this mortuary assemblage. A relatively recent addition to our documentation protocol was vessel volume (see Perttula et al. 2009). Over half of the measured vessels ( 27 of 48 , or 56 percent) were $1.0 \mathrm{~L}$ or smaller, with only seven vessels larger than $2.0 \mathrm{~L}$. Some of the vessels show evidence of prior use. Sooting, charred residues, or blackening is described on 66 percent $(n=38)$ of the jars and 10 percent of the bowls $(n=6)$ in this sample.

Two bottles with broken necks show some evidence of smoothing at the breaks, perhaps for reuse as seed jars. Of the 167 vessels, 23 vessels (14 percent) are tempered with grog (crushed potsherds or dried clay), 91 (54 percent) with crushed mussel shell, 50 (30 percent) with a mixture of shell and grog, and 3 ( 2 percent) with a mixture of shell and bone or grog, shell, and bone. Of the total, 142 (85 percent) are decorated, while 25 (15 percent) are plain. Decorative treatments to vessel rim and body were coded using the descriptive or "collegiate" system (e.g., Early 1993; Kelley 1997; Rolingson and Schambach 1981; Schambach and Miller 1984). Major categories of decoration are differentiated as classes (incising, Class A and B; punctations, Class C; brushing, Class D; engraving, Class E; stamping, Class F; slipped surfaces, Class G; and appliques, Class $\mathrm{H}$ ), with named patterns and numbered design variations within each pattern. Decorated rims and bodies of coded vessels were mainly incised (35 percent of rim decorations, 66 percent of body decorations), engraved (36 percent of rim decorations, 25 percent of body decorations), and punctated (21 percent of rim decorations, 5 percent of body decorations). Surface treatment and decoration have been used to categorize vessels to recognized types, with temper as a secondary consideration. Type (and variety) names follow Early (1988, 1993, 2000a), Kelley (1997), Phillips (1970), Schambach and Miller (1984), Suhm and Jelks (1962), and Wood (1981). Friendship Engraved, Foster TrailedIncised, Keno Trailed, Hodges Engraved, Military Road Incised, and Sandford Punctated were the most frequent types recorded (Table 5).

Using published information on the temporal distribution of Caddo ceramic types from Hardman (Early 1993) and other sites in the region, as well as data on the vessels within each grave lot and site, a ceramic seriation was created. A simplified version (that does not

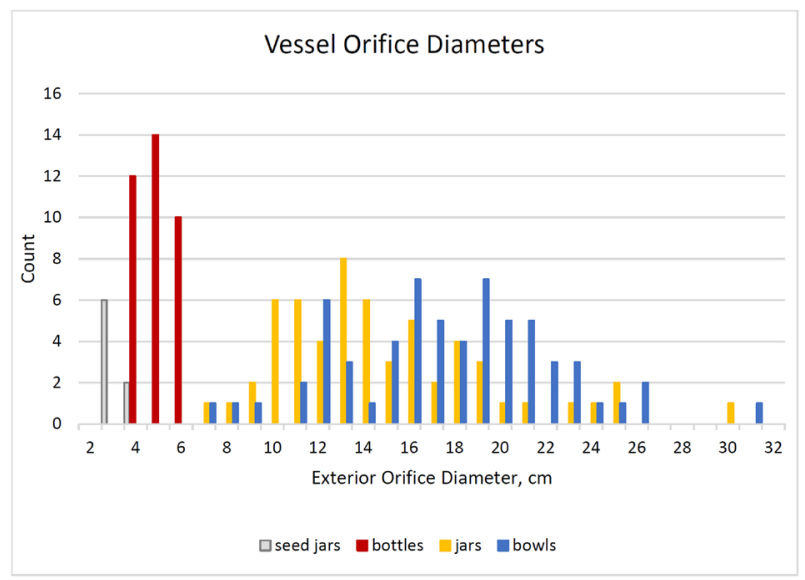

Figure 2. Size distribution of bottles, bowls, jars, and seed jars using vessel orifice diameters.

\begin{tabular}{lr} 
Type & Count \\
\hline Adair/Belcher Engraved & 1 \\
Bailey Engraved & 1 \\
Belcher Engraved (incl. var. Belcher, Soda Lake) & 3 \\
Blakely Engraved (incl. var. Blakely, Witherspoon) & 3 \\
Caney Punctated (variant?) & 1 \\
Cook Engraved & 2 \\
Crockett Curvilinear Incised (similar) & 1 \\
DeRoche Incised (incl. var. Central) & 4 \\
Emory Punctated-Incised? & 1 \\
Foster Trailed-Incised (incl. var. Dobson, Foster ) & 15 \\
Foster/Caney?, Foster/Cowhide?, Foster/Pease? & 4 \\
Friendship Engraved (incl. var. Freeman, Meador, Trigg?) & 15 \\
Hardman Engraved (similar) & 1 \\
Hempstead Engraved & 4 \\
Hodges Engraved (incl. var. Hodges, Nix ) & 12 \\
Karnack Brushed-Incised (incl. var. Fish Bayou, Midway) & 4 \\
Keno Trailed (incl. var. Curtis, Red Hill) & 12 \\
Keno related, Keno/Wallace? & 6 \\
Maddox Engraved? & 1 \\
Means Engraved, Means/Cook variant? & 3 \\
Military Road Incised, MRI variant? & 11 \\
Mound Tract Incised/Brushed (Cowhide Stamped) & 1 \\
Natchitoches/Hodges Engraved & 1 \\
Old Town Red? & 1 \\
Owens Punctated (similar) & 1 \\
Ripley Engraved? & 1 \\
Sandford Punctated & 6 \\
Sinner Linear Punctated & 1 \\
untyped vessels & 50 \\
\hline
\end{tabular}

Table 5. Listing of Vessels by Type.

include all the vessels) of the grave lot seriation for the eight sites is shown in Table 6. While some vessels may have been curated or heirloomed, it is likely that most of the vessels and other objects placed in a grave were in use at the same time.

In the Middle Ouachita River valley, grog temper was common throughout the Caddo period, but mussel shell appears as a temper during the MidOuachita phase, beginning in the late 1300s to early 1400s (Early 1993; Perttula et al. 2011). A set of 


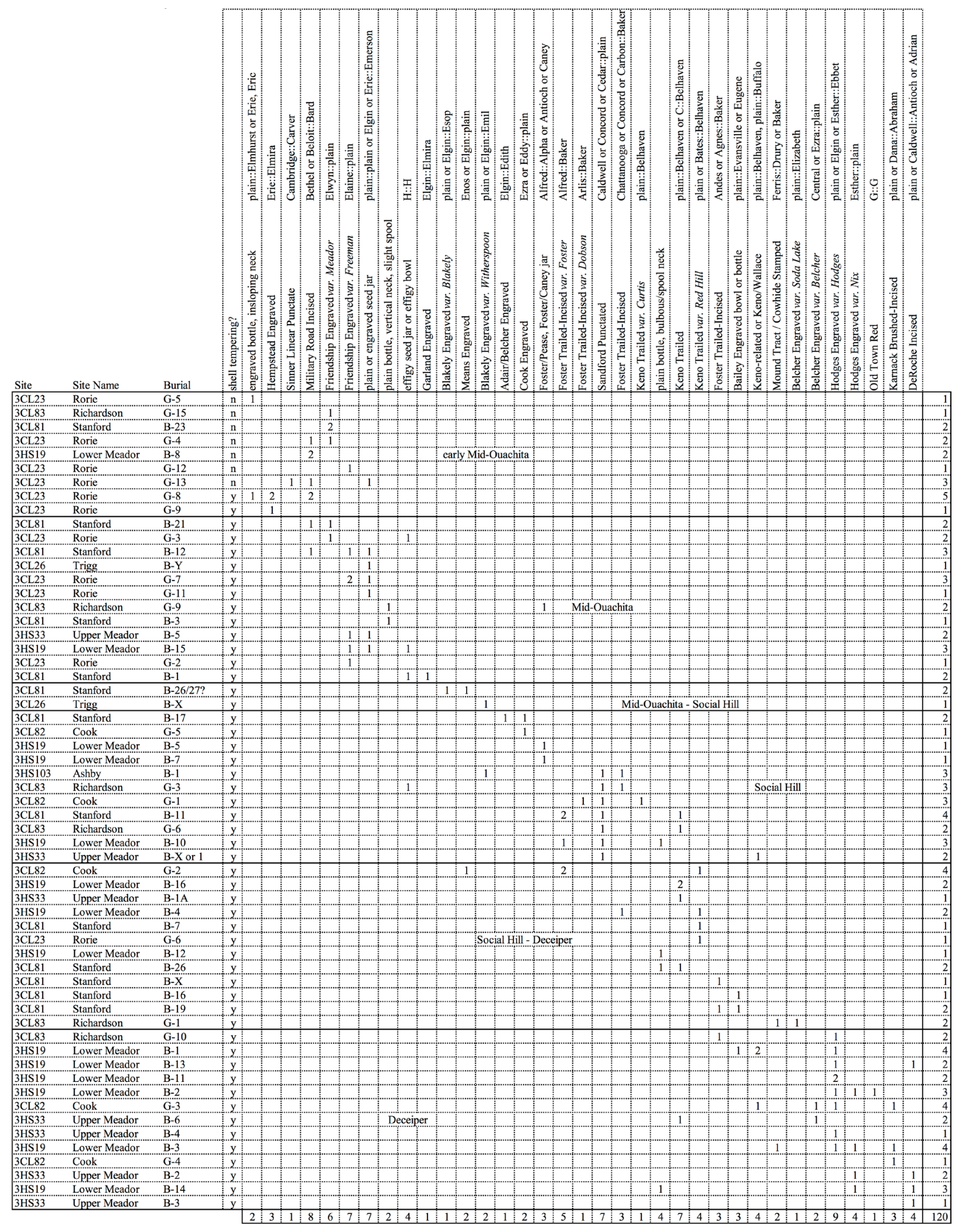

Table 6. Grave Lot Seriation using Ceramic Vessels. 
early Mid-Ouachita phase (or transitional East - MidOuachita phases) grave lots had only grog-tempered vessels, as well as early types such as a bottle engraved with a Crockett Curvilinear-Incised design, a Sinner Linear Punctate jar, and Hempstead Engraved bowls, along with more common Mid-Ouachita phase types, such as Military Road Incised jars and Friendship Engraved carinated bowls (Figure 3). East Incised, a characteristic East phase type, was not present. Sinner Linear-Punctated and Friendship Engraved vessels were found in the same grave lot at Ferguson (3HE63) as well (Chowdhury 2018). Plain and engraved seed jars (Watermelon Island seed-jars [Hodges and Hodges 1945]) were characteristic of Mid-Ouachita phase grave lots at the sites in this study.

Few Blakely Engraved bottles, either var. Blakely with diagonal lines or var. Witherspoon with vertical lines (Early 1993:87; Hodges and Hodges 1945) were found at these sites. At Hardman, a Blakely Engraved var. Witherspoon bottle was found in a terminal Mid-Ouachita phase grave with Friendship Engraved var. Freeman, Garland Engraved, Hardman Engraved, and Cook Engraved bowls, a Belcher Engraved and a plain bottle, and a Caney Punctated jar (Early 1993:87, 112). Here, a Blakely Engraved var. Blakely bottle was found in a late Mid-Ouachita to Social Hill phase grave lot from Stanford with a Means Engraved effigy bowl and an untyped engraved bowl (Trubitt 2017:Figure 1). Blakely Engraved var. Witherspoon bottles were found in grave lots from Trigg (with an untyped incised jar) and Ashby (Figure 4, with a Sandford Punctated bowl, a Foster Trailed-Incised jar, and two untyped incised jars), the latter types used as Social Hill phase markers.

Foster Trailed-Incised jars and Keno Trailed bottles, each showing a range of patterns and designs, were found in Social Hill phase grave lots, with some variants extending into the Deceiper phase. Sandford Punctated, a bowl form found at Ouachita Mountains sites (Starr 2017; Wood 1981), is represented by six vessels in the JEC Hodges Collection. It may replace Cook Engraved as a bowl form later in the Social Hill phase. Hodges Engraved bottles and bowls were used in the seriation as Deceiper phase markers, along with Karnack Brushed-Incised and DeRoche Incised jars (Figure 5). Types such as Belcher Engraved and Old
Town Red continue the trend of pottery types coming from outside the region during the Deceiper phase, a trend identified at Hardman (Early 1993:95-96, 226).

Bottle shapes clearly changed through time and may vary within a decorative type as well. Figure 6 shows examples of the change from bottles with insloping necks (early Mid-Ouachita phase) to vertical necks with slightly everted lips (Mid-Ouachita phase), to bulbous necks with everted lips or "spool" shape (Social Hill and Deceiper phases), to the "spool" neck seen in some Deceiper phase bottles.

At Hardman, Early (1993:Table 15) found a shift in vessel forms used in grave lots through time. Earlier grave lots (Mid-Ouachita phase) had a predominance of carinated bowls relative to bottles, while later grave lots (Social Hill and Deceiper phases) had a predominance of bottles relative to carinated bowls. This pattern is also seen in the analyzed vessels from grave lots in this study (Figure 7), although there is not as great a difference here, perhaps because some vessels from Huddleston grave lots were not collected or have not been identified in documented collections.

\section{Other Artifacts}

Several of the Huddleston grave lots had objects other than ceramic vessels. The few arrow points came from grave lots identified as Social Hill or Deceiper phase deposits. From Upper Meador Burial 1 were seven arrow points (Figure 8; one typed as Bassett, three with side notches and incurved bases typed as Maud var. Hopper, two with slight side notches and straight bases typed as Maud or Washita, and one triangular Maud var. Maud or Fresno/Talco point with serrated blade edges and straight to slightly incurved base). Most were made using novaculite. Upper Meador Burial 6 had a Gary var. Camden knife made using novaculite. Lower Meador Burial 4 had four novaculite arrow points, all of different types (a small contracting-stemmed Gary var. Camden point, a corner-notched Womble point, a Maud var. Maud point, and a Bassett point). This grave also contained three polished and socketed antler or bone points (and an unmodified? fish? bone). Similar point types are found in Late Caddo period contexts at sites elsewhere in southwest Arkansas (Early 1988; Kay 1984). Gary var. Camden points (Schambach 1998) may 

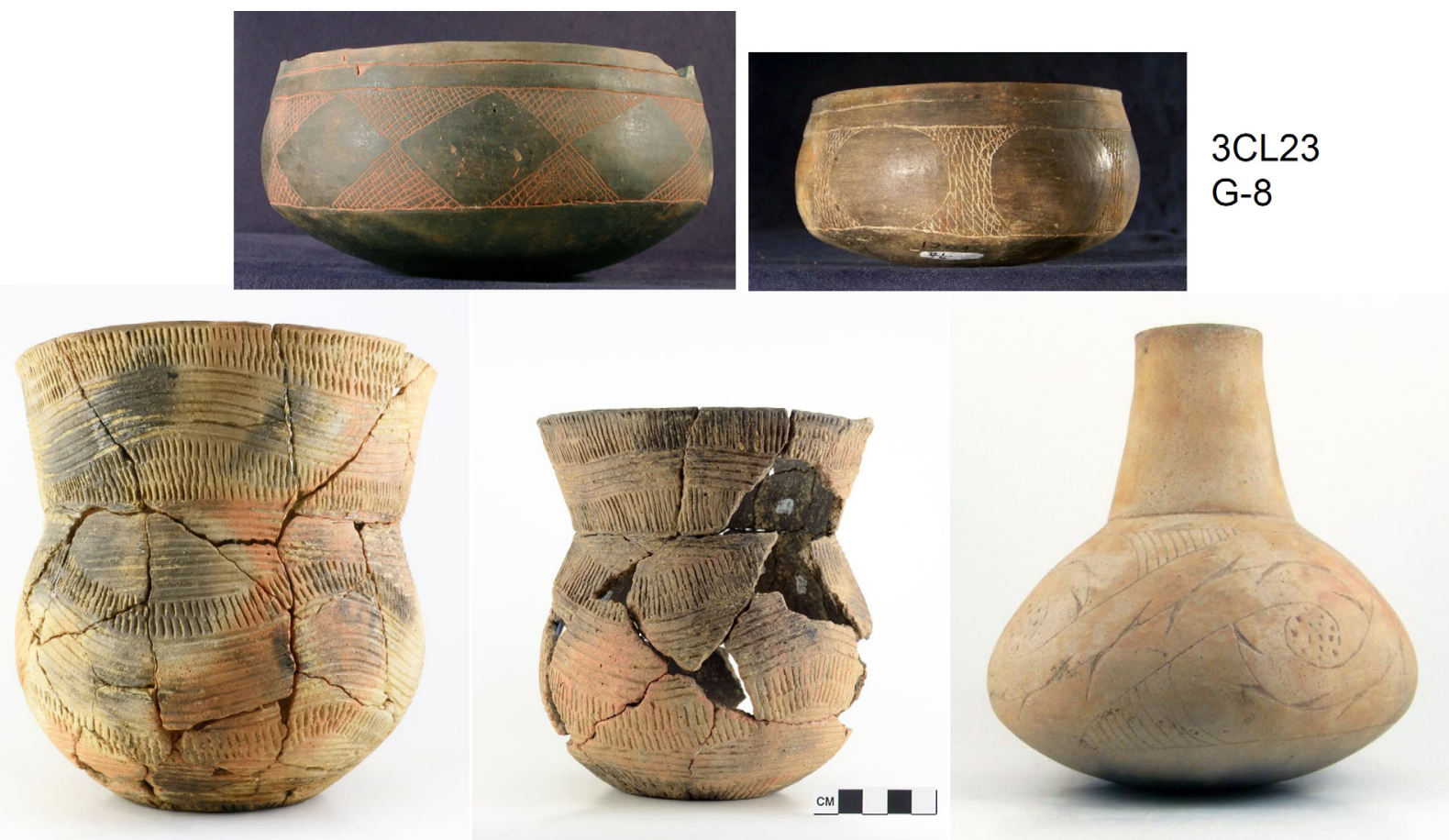

Figure 3. Ceramic vessels from an early Mid-Ouachita phase grave lot (Rorie Grave 8, 77-1/8-2, 4, 5, 16, 26), with Military Road Incised jars, Hempstead Engraved bowls, and a bottle engraved with a design similar to Crockett Curvilinear Incised.

$3 \mathrm{HS} 103$

B-1

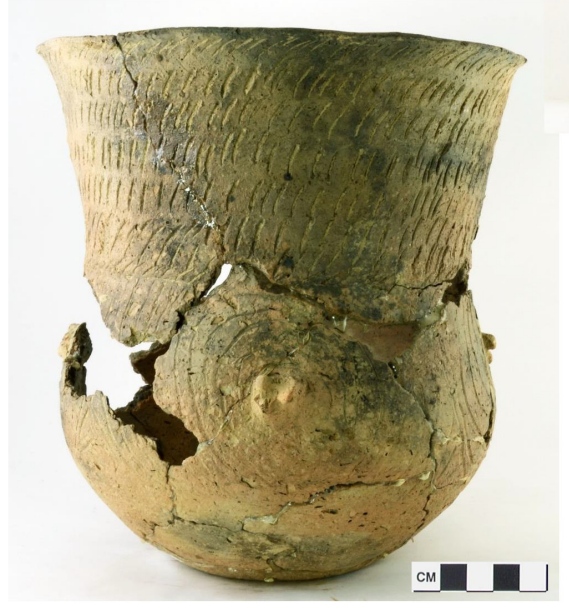

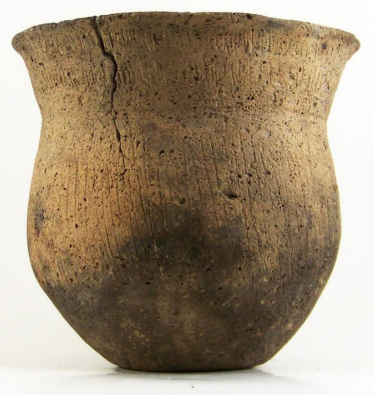
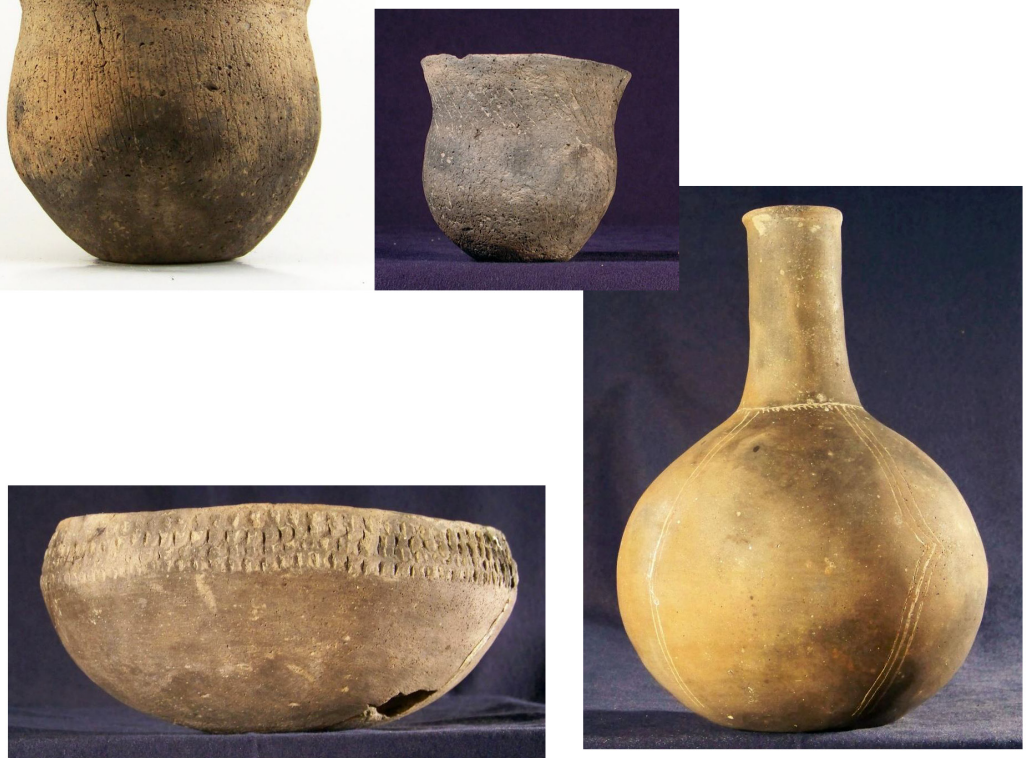

Figure 4. Ceramic vessels from a Social Hill phase grave lot (Ashby Burial 1, 77-1/22-1, 2, 3, 4, 5), with a Foster Trailed-Incised and two untyped incised jars, a Sandford Punctated bowl, and a Blakely Engraved var. Witherspoon bottle. 

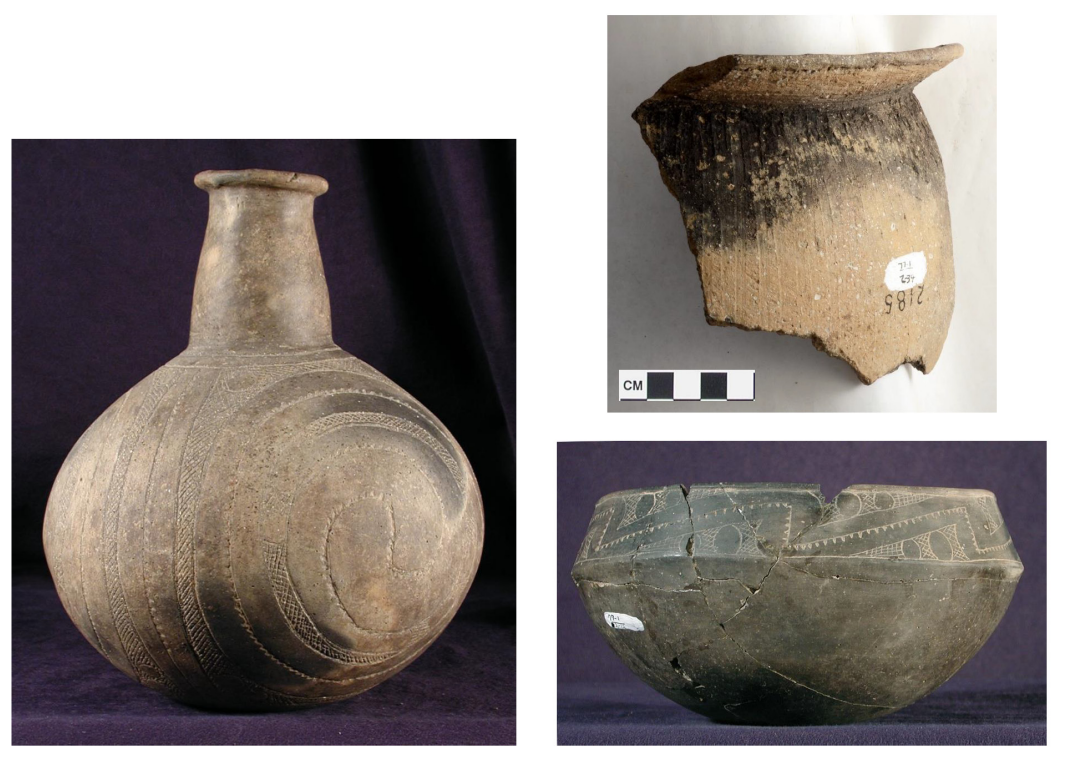

\section{$3 \mathrm{HS} 19$ \\ B-3}

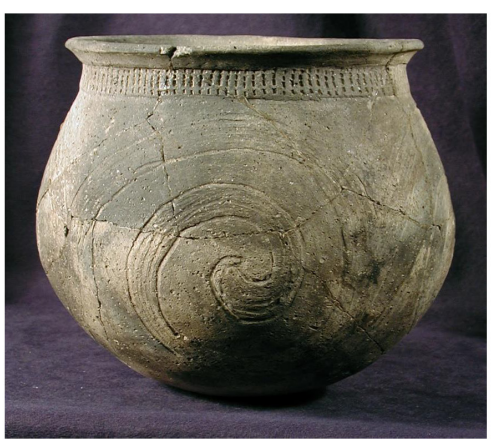

Figure 5. Ceramic vessels from a Deceiper phase grave lot (Lower Meador Burial 3, 77-1/2-12, 34, 35, 50), with a Hodges Engraved var. Hodges bottle and var. Nix bowl, a Mound Tract Incised/Brushed jar, and a large sherd from a Karnack Brushed-Incised var. Fish Bayou jar.
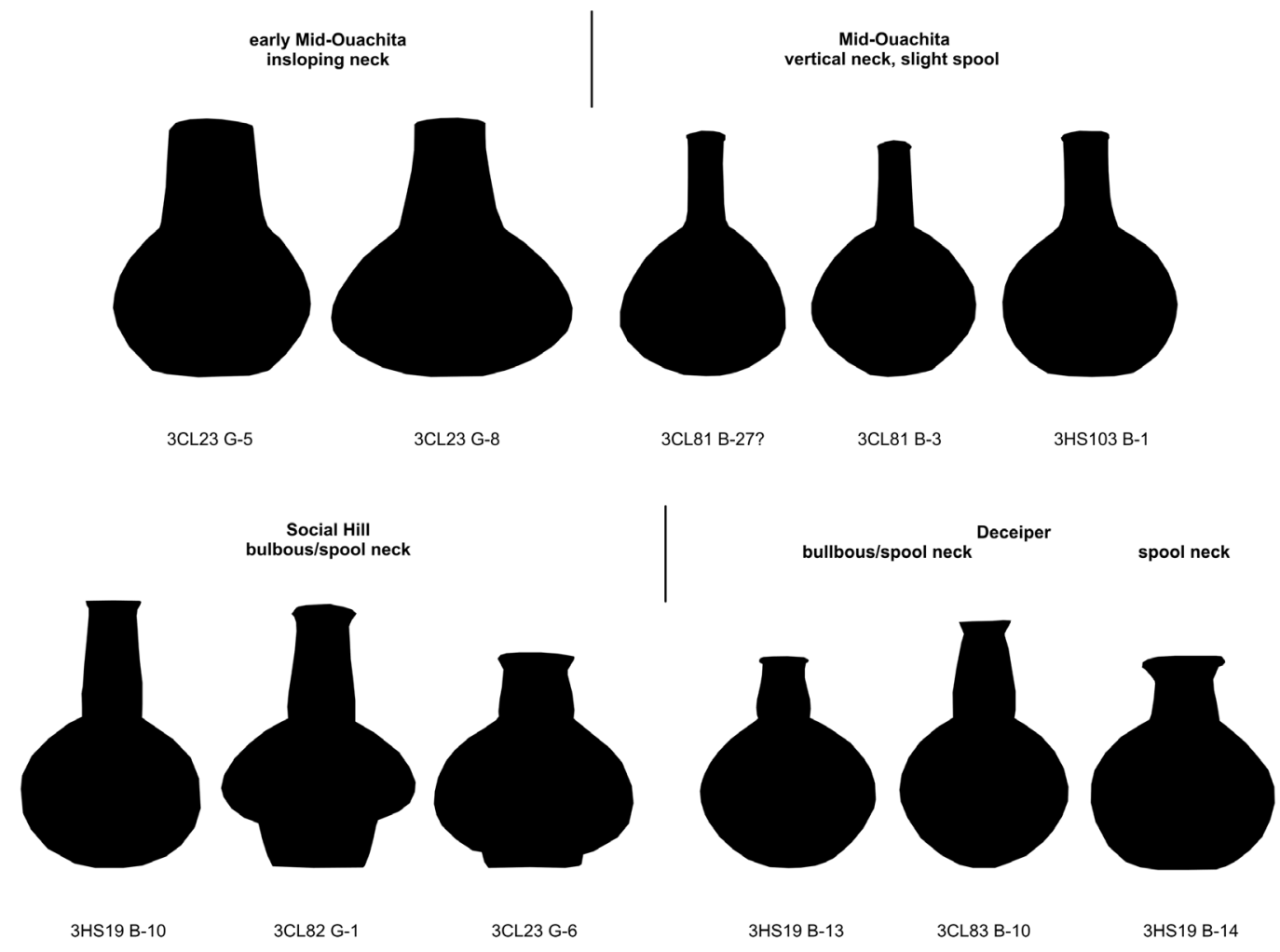

Figure 6. Changes in bottle neck shape through time (bottles are not all shown at the same scale; instead, images were resized to keep body heights consistent). 


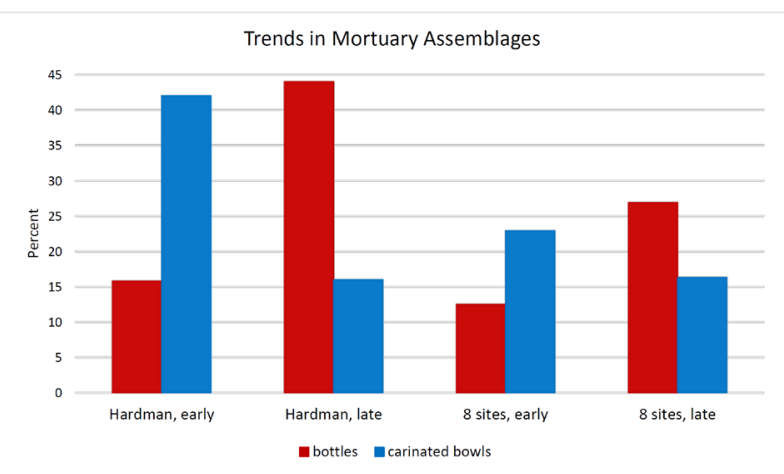

Figure 7. Trends in deposition of bottles and carinated bowls in early (Mid-Ouachita phase) and late (Social Hill, Deceiper phases) mortuary assemblages, comparing analyzed vessels Hardman (Early 1993:Table 15) with the eight sites in this study.

be heirlooms or the type may have been in use as a knife long after the Woodland period.

A large petaloid celt (19.8 cm in length), pecked and polished from fine-grained igneous rock, came from Lower Meador Burial 12. Disc beads ( $\mathrm{n}=17$ beads plus fragments, originally catalogued as 22 beads) of marine shell (most likely Busycon sp., whelk) were found in Lower Meador Burial 1 (Huddleston noted they were with a child's skeleton). Measurable beads ranged from 7.7-10.0 $\mathrm{mm}$ in diameter, 5.3-9.4 $\mathrm{mm}$ in length, with $2.7-4.5 \mathrm{~mm}$ perforations. Marine shell, shaped into a variety of ornaments or cut for use as cups, has been found in mortuary contexts across the Caddo Area (Girard et al. 2014:94-99). A deer ulna awl and unmodified freshwater mussel shell (Megalonaias nervosa, washboard) was found in Lower Meador Burial 7. A ceramic pipe came from Lower Meador Burial 8. Its loop style is characteristic of the MidOuachita phase (Early 2002b). The individual in Richardson Grave 10 was buried with two pulley-shaped unperforated earspools made of ceramic tempered with fine shell and grog. These earspools have smooth/plain outer and inner faces, and inner flanges that are only slightly smaller than outer flanges (inner $3.4-3.5 \mathrm{~cm}$ diameter, outer 3.8-3.9 cm diameter). Earspools from sites in southwest Arkansas were more often made of stone than ceramic (e.g., Bohannon 1973; Early 1988).

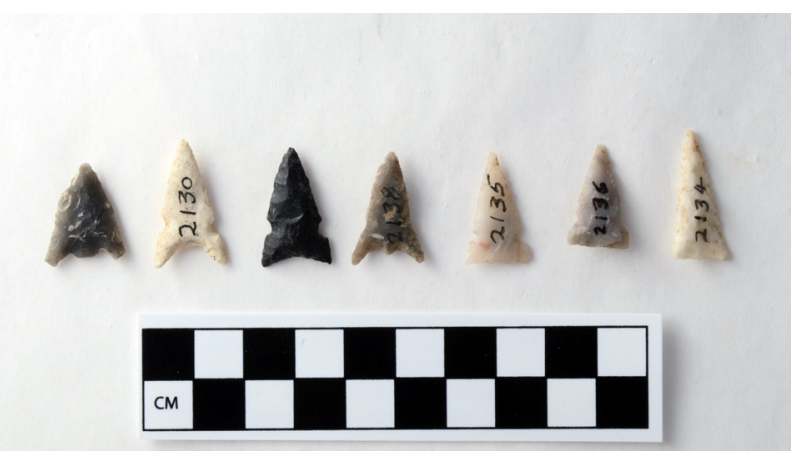

Figure 8. Arrow points from Social Hill phase Upper Meador Burial X or 1 (77-1/4-8, Bassett, Maud var. Hopper, Maud or Washita, and Maud var. Maud or Fresno/Talco point).

\section{Discussion and Conclusions}

Based on Huddleston's notes and analysis of artifacts from grave lots at Trigg and Cook, Upper Meador and the nearby Ashby site, Lower Meador and the Stanford, Richardson, and Rorie site cluster were inhabited and used for community burials during the Mid-Ouachita, Social Hill, and Deceiper phases (A.D. 1350-1700). While most of the sites show a temporal range, more of the grave lots from Stanford, Richardson, and Rorie dated stylistically to the Mid-Ouachita phase, while the majority of grave lots from Cook, Upper Meador and Ashby, and Lower Meador dated to the Social Hill and Deceiper phases (Table 7).

In all, the majority of the grave lots from the eight sites ( 38 of 62 , or 61 percent) were assigned to the Social Hill or Deceiper phases (A.D. 1500-1700). If we shift our view from the mortuary assemblages to the communities living in the Middle Ouachita River valley in the past, this indicates a populous region during the Social Hill and Deceiper phases (or, alternatively, a large number of deaths at this time). The Middle Ouachita River valley was not depopulated following the Spanish expedition of Hernando de Soto in 1541-1542. Instead, the major changes came at the beginning of the 1700s (see also Perttula 2017:132). Sites were abandoned and Caddo communities moved out of the area, presumably joining related groups further to the south. Archeological evidence for European-made trade goods is lacking from sites in the Middle Ouachita River valley (Early 2000b), whereas Historic Caddo sites continued into the eighteenth century in northwest Louisiana and east 


\begin{tabular}{|c|c|c|c|c|c|c|c|c|c|c|}
\hline & \multirow[b]{2}{*}{ Phase } & \multicolumn{2}{|c|}{ Trigg \& Cook } & \multicolumn{2}{|c|}{ Upper Meador \& Ashby } & \multirow{2}{*}{$\begin{array}{l}\text { Lower } \\
\text { Meador } \\
\text { 3HS19 }\end{array}$} & \multicolumn{3}{|c|}{ Stanford, Richardson \& Rorie } & \multirow[b]{2}{*}{ Total } \\
\hline & & $3 \mathrm{CL} 26$ & 3CL82 & $3 \mathrm{HS} 33$ & $3 \mathrm{HS} 103$ & & 3CL81 & 3CL83 & 3CL23 & \\
\hline \multirow{3}{*}{ 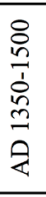 } & $\begin{array}{l}\text { early Mid- } \\
\text { Ouachita }\end{array}$ & & & & & 1 & 1 & & 5 & 7 \\
\hline & $\begin{array}{c}\text { Mid- } \\
\text { Ouachita }\end{array}$ & 1 & & 1 & & 1 & 5 & 2 & 5 & 15 \\
\hline & $\begin{array}{l}\text { Mid-Oua./ } \\
\text { Social Hill }\end{array}$ & 1 & & & & & 1 & & & 2 \\
\hline \multirow{4}{*}{ 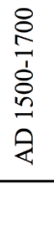 } & Social Hill & & 2 & 1 & 1 & 5 & 2 & 2 & & 13 \\
\hline & $\begin{array}{l}\text { Social Hill/ } \\
\text { Deceiper }\end{array}$ & & 1 & 1 & & 3 & 5 & 1 & 1 & 12 \\
\hline & Deceiper & & 2 & 4 & & 6 & & 1 & & 13 \\
\hline & Total & 2 & 5 & 7 & 1 & 16 & 14 & 6 & 11 & 62 \\
\hline
\end{tabular}

Table 7. Distribution of Grave Lots by Phase.

Texas (Girard et al. 2014:122-130; Perttula 2017:217246). Perhaps Caddos in the Middle Ouachita River valley moved southward to participate in trading opportunities with the French and Spanish colonists.

This paper describes ceramic vessels and other artifacts excavated from eight sites along the Caddo and Ouachita rivers in the vicinity of Arkadelphia, Arkansas by amateur archeologist Vere Huddleston between 1937 and 1944. These artifacts later became part of the Joint Educational Consortium's (JEC) Hodges Collection. The documented artifacts show that these archeological sites - Trigg (3CL26) and Cook (3CL82), Upper Meador (3HS33) and Ashby (3HS103), Lower Meador (3HS19), and Stanford (3CL81), Richardson (3CL83), and Rorie (3CL23) - were ancestral Caddo Indian communities.

Based on Huddleston's notes on burials he dug at these sites, we can link artifacts (and in some cases, human remains) to specific graves. A total of 167 vessels and 44 other objects ( 12 arrow points, a celt, a pipe, two ceramic earspools, and 28 bone/shell artifacts) in the JEC Hodges Collection from these eight sites have been documented in this study. Most of these are from 62 grave lots that can be dated, using stylistic and technological characteristics of the artifacts, to the Mid-Ouachita, Social Hill, and Deceiper phases (ca. A.D. 1350-1700). Of these 62 grave lots, 11 (eight from Lower Meador and three from Richardson) are associated with human remains in the collection (another two grave lots have human remains but no artifacts, and human remains from Stanford and Upper Meador are from unknown context). These remains and associated funerary objects (34 vessels and 36 other objects) have been transferred to the ARAS-CO for NAGPRA documentation. Artifacts from the other 51 grave lots are considered unassociated funerary objects (118 vessels and eight other objects). Context is unclear for 15 vessels.

This study has been undertaken in part to advise the Joint Educational Consortium and the Caddo Nation of Oklahoma, and provide information for the NAGPRA process. Analysis of stylistic and technological characteristics of the ceramic vessels resulted in a seriation of grave lots. Reconstruction of grave lots provides information about artifacts that were deposited contemporaneously, even if they were not all made at the same time or in the same community (in several instances, grave lots contained vessels that may have been kept as heirlooms or are types found more often in other regions). The results reinforce patterns recognized by Early at the nearby Hardman site, but also point to some new findings (for example, Sandford Punctated appears as a common Social Hill phase type at these sites). The artifacts in the grave lots indicate that there were active Caddo communities in this part of the Middle Ouachita River valley between at least A.D. 1350 and 1700. While other regions of Arkansas saw populations decrease after the de Soto expedition, the sites in this study indicate local communities continued until the end of the seventeenth century.

\section{Acknowledgments}

The Joint Educational Consortium's Hodges Collection (which includes Huddleston's materials) is curated at 
Henderson State University in Arkadelphia, Arkansas (human remains and associated funerary objects are at the Arkansas Archeological Survey's Fayetteville office for NAPGRA documentation and publication). There are a few vessels from Huddleston grave lots that are in HSU Museum collections (currently on long-term loan to the Historic Arkansas Museum in Little Rock). Original Huddleston and Hodges notes and records are curated at HSU Archives, with copies on file at the ARAS-HSU research station. The documentation of vessels in the Huddleston grave lots in the JEC Hodges Collection follows a protocol originally developed by Ann Early. Documentation has taken many years and I appreciate the help of the ARAS-HSU station assistants and lab volunteers who have worked with me on the analysis and photography, including Milton Hughes, Kate Wright, Matthew Reynolds, Jeffrey Gaskin, Vanessa Hanvey, Katie Leslie, Chelsea Cinotto, Mary Ann Goodman, Janice Fisher, Florence Davis, Judy Thye, John Chapman, Jim Hudgins, and Patsy Mathews. Vessel documentation during 2006-2007 was funded in part by a grant from the Ross Foundation. Robert Scott (ARAS-UAPB) identified the mussel shell from Lower Meador Burial 7 from a photograph. Thank you to Ann Early and Sarah Hunt Shepard, and to an anonymous reviewer, for comments on earlier drafts of this paper.

\section{References Cited}

Bohannon, Charles F.

1973 Excavations at the Mineral Springs Site, Howard County, Arkansas. Research Series No. 5. Arkansas Archeological Survey, Fayetteville.

Chowdhury, Pritam

2018 Middle Caddo Whole Vessels from the Ferguson Site (3HE63). Caddo Archeology Journal 28:6091.

Early, Ann M.

1986 Dr. Thomas L. Hodges and his Contribution to Arkansas Archeology. The Arkansas Archeologist 23-24:1-9.
1988 Standridge: Caddoan Settlement in a Mountain Environment. Research Series No. 29. Arkansas Archeological Survey, Fayetteville.

2000a Ceramics. In Data Recovery at the Helm Site, 3HS449, Hot Spring County, Arkansas, by Robert H. Lafferty III, Ann Early, Michael C. Sierzchula, H. Cassandra Hill, Gina S. Powell and Neal H. Lopinot, Linda Scott Cummings, Susan L. Scott, Samuel K. Nash, and Timothy K. Perttula, pp. 69-121. MCRA Report 2000-1 (submitted to the Arkansas Highway and Transportation Department), Mid-Continental Research Associates, Lowell, Arkansas.

2000b The Caddos of the Trans-Mississippi South. In Indians of the Greater Southeast: Historical Archaeology and Ethnohistory, edited by Bonnie G. McEwan, pp. 122-141. University Press of Florida, Gainesville.

2002a Arkansas Prehistory and History in Review: The East Phase. Field Notes: Newsletter of the Arkansas Archeological Society 304:4-8.

2002b The Mid-Ouachita Phase. Field Notes: Newsletter of the Arkansas Archeological Society 305:10-13.

2002c Arkansas Prehistory and History in Review: The Social Hill Phase. Field Notes: Newsletter of the Arkansas Archeological Society 306:10-13.

2002d Arkansas Prehistory and History in Review: Deceiper Phase. Field Notes: Newsletter of the Arkansas Archeological Society 307:8-11.

2012 Form and Structure in Prehistoric Caddo Pottery Design. In The Archaeology of the Caddo, edited by Timothy K. Perttula and Chester P. Walker, pp. 26-46. University of Nebraska Press, Lincoln.

Early, Ann M. (editor)

1993 Caddoan Saltmakers in the Ouachita Valley: The Hardman Site. Research Series No. 43. Arkansas Archeological Survey, Fayetteville. 
Girard, Jeffrey S., Timothy K. Perttula, and Mary Beth Trubitt

2014 Caddo Connections: Cultural Interactions within and beyond the Caddo World. Rowman and Littlefield, Lanham, Maryland.

Hodges, T. L., and Mrs. [Charlotte Hodges]

1945 Suggestion for Identification of Certain MidOuachita Pottery as Cahinnio Caddo. Bulletin of the Texas Archeological and Paleontological Society 16:98-116.

Jeter, Marvin D. (editor)

2009 Edward Palmer's Arkansas Mounds. Reprinted. University of Alabama Press, Tuscaloosa. Originally published 1990, University of Arkansas Press, Fayetteville.

Kay, Marvin

1984 Late Caddo Subtractive Technology in the Red River Basin. In Cedar Grove: An Interdisciplinary Investigation of a Late Caddo Farmstead in the Red River Valley, edited by Neal L. Trubowitz, pp. 174-206. Research Series No. 23. Arkansas Archeological Survey, Fayetteville.

Kelley, David B., editor

1997 Two Caddoan Farmsteads in the Red River Valley: The Archeology of the McLelland and Joe Clark Sites. Research Series No. 51. Arkansas Archeological Survey, Fayetteville.

McKinnon, Duncan P.

2015 Zoomorphic Effigy Pendants: An Examination of Style, Medium, and Distribution in the Caddo Area. Southeastern Archaeology 34(2):116-135.

Perttula, Timothy K.

2017 Caddo Landscapes in the East Texas Forests. American Landscapes series, Oxbow Books, Havertown, Pennsylvania.
Perttula, Timothy K., Bo Nelson, Mark Walters, Robert Cast, and Bobby Gonzalez

2009 Documentation of Caddo Funerary Objects in the Gilcrease Museum Collections. Historic Preservation Program, Caddo Nation of Oklahoma, Binger, and Special Publication No. 12, Friends of Northeast Texas Archaeology, Pittsburg.

Perttula, Timothy K., Mary Beth Trubitt, and Jeffrey S. Girard

2011 The Use of Shell-Tempered Pottery in the Caddo Area of the Southeastern United States. Southeastern Archaeology 30(2):242-267.

Phillips, Philip

1939 Introduction to the Archaeology of the Mississippi Valley. PhD dissertation, Department of Anthropology, Harvard University, Cambridge.

1970 Archaeological Survey in the Lower Yazoo Basin, Mississippi, 1949-1955. Papers of the Peabody Museum of Archaeology and Ethnology Vol. 60. Peabody Museum, Harvard University, Cambridge, Massachusetts.

Rolingson, Martha A., and Frank F. Schambach

1981 The Shallow Lake Site (3UN9/52) and its Place in Regional Prehistory. Research Series No. 12. Arkansas Archeological Survey, Fayetteville.

Schambach, Frank F.

1998 Pre-Caddoan Cultures in the Trans-Mississippi South. Research Series No. 53. Arkansas Archeological Survey, Fayetteville.

Schambach, Frank F., and John E. Miller 1984 A Description and Analysis of the Ceramics. In Cedar Grove: An Interdisciplinary Investigation of a Late Caddo Farmstead in the Red River Valley, edited by Neal L. Trubowitz, pp. 109-170. Research Series No. 23. Arkansas Archeological Survey, Fayetteville. 
Starr, Joanne DeMaio

2017 The Adair Site: Caddo Relations through Ceramic Analysis. Caddo Archeology Journal 27:27-35.

Suhm, Dee Ann, and Edward B. Jelks, editors

1962 Handbook of Texas Archeology: Type

Descriptions. Special Publication No. 1, Texas

Archeological Society, and Bulletin No. 4, Texas

Memorial Museum, Austin.

Thomas, Cyrus

1894 Report on the Mound Explorations of the Bureau of Ethnology. In Twelfth Annual Report of the Bureau of Ethnology to the Secretary of the Smithsonian Institution, 1890-'91, edited by J. W. Powell, pp. 1-730. Government Printing Office, Washington, DC.

Trubitt, Mary Beth

2003 Archeological Salvage at the Rorie Place (3CL23). Field Notes: Newsletter of the Arkansas Archeological Society 312:3-7.

2016a Documenting Caddo Pottery: The JEC Hodges Collection. Research page on Arkansas Archeological Survey website. Electronic document, http://archeology.uark.edu/learndiscover/current-research/hodges/, accessed May 29, 2018. 2016b The Other "Star" Bottle from Menard. Field Notes: Newsletter of the Arkansas Archeological Society 391:6-7.

2017 Effigy Pottery in the Joint Educational Consortium's Hodges Collection. Caddo Archeology Journal 27:51-93.

Trubitt, Mary Beth, and Linda Evans

2015 Revisiting a Historic Manuscript: Vere Huddleston's Report on East Place (3CL21) Excavations. Caddo Archeology Journal 25:73144.

Walker, Leslie

2014 Liminal River: Art, Agency and Cultural Transformation along the Protohistoric Arkansas River. PhD dissertation, Department of Anthropology, University of Arkansas, Fayetteville.

Wood, W. Raymond, with Ann M. Early

1981 The Poole Site, 3GA3. The Arkansas Archeologist, Bulletin of the Arkansas Archeological Society 22:7-64. 
JEC Hodges Collection, 77-1

Artifact No. / Old Cat. No. / Site No.

Vessel Form

ARAS/HSU Digital Photo

Type

Decoration

Rim :: Body

Paste

Temper

Core Color (Munsell)

Exterior Color (Munsell)

Interior Color (Munsell)

Vessel Overall Height $(\mathrm{cm})$

Vessel Overall Weight (g)

Vessel Maximum Diameter $(\mathrm{cm})$

Volume (liters)

Usewear/Sooting/Condition

Shape/Description

Lip Treatment/Shape/Angle/Thickness

Orifice Exterior Diameter $(\mathrm{cm})$

Rim/Neck Height (cm)

Neck Mid/Base Exterior Diameter $(\mathrm{cm})$

Rim/Neck Shape/Angle

Rim/Neck Surface/Decorative Treatment

Rim/Neck/Body Thickness (cm)

Body Shape

Body Maximum Diameter (cm)

Body Height (cm)

Body Surface/Decorative Treatment

Base Shape/Curvature

Base Diameter/Height $(\mathrm{cm})$

Base Surface/Decorative Treatment

Appendages/Handles
2-4 / 2187 / 3HS19

bottle

$1813, \mathrm{~N} 22949$

Keno Trailed var. Red Hill

incised

plain :: Belhaven 31

SOFT, SMOOTH, SLIGHTLY CRUMBLY, MICA IN PASTE

SHELL (PARTLY LEACHED), FINE \& ABUNDANT + GROG, FINE \& MED ABUNDANT

GRAY (10YR5/1) (CORE MODE B)

PALE BROWN (10YR6/3), VERY DARK GRAY (10YR3/1)

DARK GRAY (10YR4/1)

17.0

702

16.7

BASE SHOWS ABRASIONS, HEAVILY WORN, BOTTLE LIP ALSO LOOKS WORN/ERODED; FIRECLOUDING ON BODY; RECONSTRUCTION IN PAST; 2187 INKED ON LOWER BODY

SQUAT GLOBULAR BOTTLE WITH SHORT CYLINDRICAL NECK, FLARED RIM, OUTSLANTING PEDESTAL

ROUNDED, EVERTED, 0.3

5.7

3.8

$5.1 / 5.2$

SPOOL

SMOOTHED \& POLISHED

0.4

LOW-WAIST

16.7

12.0

SMOOTHED \& POLISHED; CURVILINEAR INCISING, NESTED LINES, SWIRLS, 1MM LINES, 4MM APART, REPEATS 4X AROUND UPPER BODY

CIRCULAR/FLAT, PEDESTAL

$6.6-8.0 / 1.2$

Associated Funerary Object; Huddleston Burial 4, 3/26/1940; Social Hill - Deceiper phase 
Artifact No. / Old Cat. No. / Site No.

Vessel Form

ARAS/HSU Digital Photo

Type

Decoration

Rim :: Body

Paste

Temper

Core Color (Munsell)

Exterior Color (Munsell)

Interior Color (Munsell)

Vessel Overall Height $(\mathrm{cm})$

Vessel Overall Weight (g)

Vessel Maximum Diameter (cm)

Volume (liters)

Usewear/Sooting/Condition

Shape/Description

Lip Treatment/Shape/Angle/Thickness

Orifice Exterior Diameter $(\mathrm{cm})$

Rim/Neck Height (cm)

Neck Mid/Base Exterior Diameter (cm)

Rim/Neck Shape/Angle

Rim/Neck Surface/Decorative Treatment

Rim/Neck/Body Thickness (cm)

Body Shape

Body Maximum Diameter (cm)

Body Height (cm)

Body Surface/Decorative Treatment

Base Shape/Curvature

Base Diameter/Height (cm)

Base Surface/Decorative Treatment

Appendages/Handles

Notes
2-5 / 2169 / 3HS19

jar, short rim

$1805, \mathrm{~N} 23120$

Keno related

incised

plain :: Belhaven 2

SOFT, COMPACT

SHELL (LEACHED), COARSE \& ABUNDANT

GRAY (7.5YR5/1) (CORE MODE F)

VERY DARK GRAY (7.5YR3/1) FIRECLOUDS, PINK (7.5YR7/3), WHITE (7.5YR8/1)

REDDISH YELLOW (7.5YR6/6), VERY PALE BROWN (10YR7/4)

6.7

81

FIRECLOUDING; CHIP IN LIP, MINOR REPAIR IN PAST; 2169 INKED NEAR BASE

SMALL GLOBULAR JAR WITH FLAT OVAL BASE AND SHORT FLARING RIM

SMOOTHED, ROUNDED, NONE, 0.3

9.5

1.3

STRAIGHT, OUTSLANTED

SMOOTHED

0.3

SUB-GLOBULAR

8.2

5.4

SMOOTHED \& BURNISHED; INCISED/TRAILED, 4MM WIDE LINES, 6-8MM APART, CURVILINEAR, INTERLOCKING SCROLLS REPEATS 5X AROUND BODY

OVAL/FLAT

$3.2 \times 2.9$

BURNISHED/PLAIN

Associated Funerary Object; Huddleston Burial 1, 3/23 or 3/24/1940 (Huddleston noted this vessel with adult in grave); Deceiper phase 
JEC Hodges Collection, 77-1

Artifact No. / Old Cat. No. / Site No.

Vessel Form

ARAS/HSU Digital Photo

Type

Decoration

Rim :: Body

Paste

Temper

Core Color (Munsell)

Exterior Color (Munsell)

Interior Color (Munsell)

Vessel Overall Height (cm)

Vessel Overall Weight (g)

Vessel Maximum Diameter (cm)

Volume (liters)

Usewear/Sooting/Condition

Shape/Description

Lip Treatment/Shape/Angle/Thickness

Orifice Exterior Diameter $(\mathrm{cm})$

Rim/Neck Height (cm)

Neck Mid/Base Exterior Diameter $(\mathrm{cm})$

Rim/Neck Shape/Angle

Rim/Neck Surface/Decorative Treatment

Rim/Neck/Body Thickness (cm)

Body Shape

Body Maximum Diameter $(\mathrm{cm})$

Body Height (cm)

Body Surface/Decorative Treatment

Base Shape/Curvature

Base Diameter/Height $(\mathrm{cm})$

Base Surface/Decorative Treatment

Appendages/Handles

Notes
2-6 / 2178 / 3HS19

bowl, simple

1207

Old Town Red?

red slipped

$\mathrm{G}:: \mathrm{G}$

SOFT, SILTY, COMPACT

SHELL (SOME LEACHED), MED TO COARSE, ABUNDANT

GRAY (10YR6/1)

RED (2.5YR4/8), REDDISH YELLOW (5YR6/6)

RED (2.5YR4/8), GRAY (10YR6/1)

4.7

68

10.9

VESSEL HAS BEEN RECONSTRUCTED WITH GLUE IN PAST, LUGS PARTIALLY BROKEN

VERY SMALL, SHALLOW BOWL, EGG-SHAPED, SLIPPED, TWO VERY SMALL LUGS (ONE ON EACH SIDE OF VESSEL)

FLATTENED, NONE (DIRECT)

10.9

STRAIGHT, OUTSLANTED

SMOOTHED SLIGHTLY, RED SLIPPED, UNEVEN (ERODED)

$0.6-0.5$

CONVEX OR ELONGATED (BODY CONTINUOUS WITH BASE)

10.1

4.7

SMOOTHED; RED SLIPPED (ERODED) INTERIOR, EXTERIOR

UNDISTINGUISHED/CONVEX

2 LUGS/TABS, 1 ON EITHER SIDE OF VESSEL AT LIP (1 BROKEN)

Huddleston Burial 2, 3/24/1940; Deceiper phase 


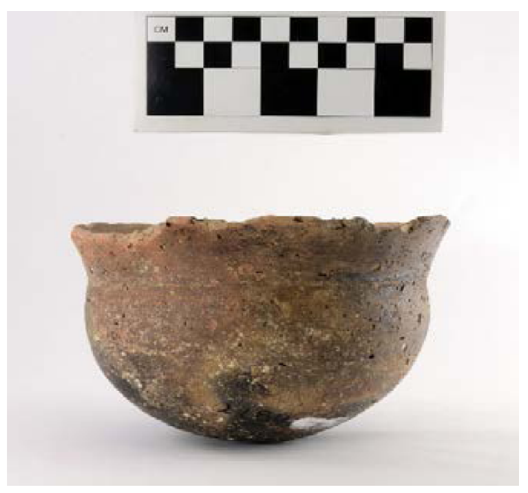

Artifact No. / Old Cat. No. / Site No.

Vessel Form

ARAS/HSU Digital Photo

Type

Decoration

Rim :: Body

Paste

Temper

Core Color (Munsell)

Exterior Color (Munsell)

Interior Color (Munsell)

Vessel Overall Height $(\mathrm{cm})$

Vessel Overall Weight (g)

Vessel Maximum Diameter $(\mathrm{cm})$

Volume (liters)

Usewear/Sooting/Condition

Shape/Description

Lip Treatment/Shape/Angle/Thickness

Orifice Exterior Diameter $(\mathrm{cm})$

Rim/Neck Height (cm)

Neck Mid/Base Exterior Diameter $(\mathrm{cm})$

Rim/Neck Shape/Angle

Rim/Neck Surface/Decorative Treatment

Rim/Neck/Body Thickness (cm)

Body Shape

Body Maximum Diameter $(\mathrm{cm})$

Body Height $(\mathrm{cm})$

Body Surface/Decorative Treatment

Base Shape/Curvature

Base Diameter/Height $(\mathrm{cm})$

Base Surface/Decorative Treatment

Appendages/Handles

Notes
$2-11 / 2180 / 3$ HS 19

jar, short rim

1831, N13153

plain

HARD, SMOOTH, COMPACT

SHELL (PARTIALLY LEACHED), coarse \& abundant

RED (2.5YR4/8), LIGHT GRAY (5YR7/1), LIGHT RED (2.5YR6/6)

BLACK (5Y2.5/1), RED (2.5YR5/8), LIGHT REDDISH BROWN (5YR6/4)

BROWN (7.5YR4/3), RED (2.5YR5/6), VERY DARK GRAY (5YR3/1)

8.5

250

13.6

0.6

ROUGHENED BASE; POSSIBLE SOOT AT RIM/BODY JUNCTURE, FIRECLOUDING PRESENT; MISSING MUCH OF RIM AND LIP, VERY ERODED; 2180 INKED ON BASE

SMALL SHORT RIM JAR OR BOWL; LINE BETWEEN BODY/RIM IS JUNCTION RATHER THAN DECORATION

SMOOTHED, ROUNDED, NONE, 0.3

13.6

2.5

Concave, Outslanted

BURNISHED

$0.6-0.5$

HIGHWAISTED

11.8

6.0

BURNISHED; PLAIN

UNDISTINGUISHED/CONVEX

BURNISHED 
JEC Hodges Collection, 77-1

Artifact No. / Old Cat. No. / Site No.

Vessel Form

ARAS/HSU Digital Photo

Type

Decoration

Rim :: Body

Paste

Temper

Core Color (Munsell)

Exterior Color (Munsell)

Interior Color (Munsell)

Vessel Overall Height $(\mathrm{cm})$

Vessel Overall Weight (g)

Vessel Maximum Diameter $(\mathrm{cm})$

Volume (liters)

Usewear/Sooting/Condition

Shape/Description

Lip Treatment/Shape/Angle/Thickness

Orifice Exterior Diameter $(\mathrm{cm})$

Rim/Neck Height (cm)

Neck Mid/Base Exterior Diameter $(\mathrm{cm})$

Rim/Neck Shape/Angle

Rim/Neck Surface/Decorative Treatment

Rim/Neck/Body Thickness (cm)

Body Shape

Body Maximum Diameter $(\mathrm{cm})$

Body Height $(\mathrm{cm})$

Body Surface/Decorative Treatment

Base Shape/Curvature

Base Diameter/Height $(\mathrm{cm})$

Base Surface/Decorative Treatment

Appendages/Handles
2-12 / 2183 / 3HS19

bowl, carinated

1177

Hodges Engraved var. Nix

engraved

Esther 12 (type) :: plain

HARD, COMPACT

GROG, FINE \& SPARSE, VERY FEW SURFACE VOIDS

LIGHT BROWNISH GRAY (10YR6/2)

BLACK (7.5YR2.5/1), BROWN (7.5YR4/2)

VERY DARK GRAY (7.5YR3/1)

9.5

18.5

VESSEL WAS RECONSTRUCTED WITH GLUE IN PAST, SEVERAL SMALL PCS MISSING

CARINATED BOWL, ROUNDED BOTTOM, ENGRAVED RIM

ROUNDED, SLIGHTLY THICKENED

16.7

3.0

STRAIGHT, INSLANTED/CARINATED

SMOOTHED \& BURNISHED, ENGRAVED-SCROLL WITH TICKED LINE, CROSSHATCHING, BALLS, REPEATED 6X AROUND RIM

$0.5-0.4$

CONVEX (CONTINUOUS BODY/BASE)

18.5

6.5

SMOOTHED \& BURNISHED; PLAIN

UNDISTINGUISHED/CONVEX

Notes

Huddleston Burial 3, 3/24/1940; Deceiper phase 
Artifact No. / Old Cat. No. / Site No.

Vessel Form

ARAS/HSU Digital Photo

Type

Decoration

Rim :: Body

Paste

Temper

Core Color (Munsell)

Exterior Color (Munsell)

Interior Color (Munsell)

Vessel Overall Height $(\mathrm{cm})$

Vessel Overall Weight (g)

Vessel Maximum Diameter $(\mathrm{cm})$

Volume (liters)

Usewear/Sooting/Condition

Shape/Description

Lip Treatment/Shape/Angle/Thickness

Orifice Exterior Diameter $(\mathrm{cm})$

Rim/Neck Height (cm)

Neck Mid/Base Exterior Diameter $(\mathrm{cm})$

Rim/Neck Shape/Angle

Rim/Neck Surface/Decorative Treatment

Rim/Neck/Body Thickness (cm)

Body Shape

Body Maximum Diameter $(\mathrm{cm})$

Body Height $(\mathrm{cm})$

Body Surface/Decorative Treatment

Base Shape/Curvature

Base Diameter/Height $(\mathrm{cm})$

Base Surface/Decorative Treatment

Appendages/Handles

Notes
2-13 / 2395 / 3HS19

bottle

1825, N20624

Keno Trailed

incised

plain :: Belhaven 6

HARD, SMOOTH, COMPACT

SHELL (PARTLY LEACHED), FINE \& ABUNDANT

PALE BROWN (10YR6/3)

DARK GRAY (10YR4/1), GRAYISH BROWN (10YR5/2), V PALE BROWN (10YR7/4)

22.7

600

13.7

NECK GLUED TO VESSEL IN PAST, SOME SHERDS MISSING (PROBE DAMAGE?), CRACKS; 2395 INKED NEAR BASE

BOTTLE, SMALL

ROUNDED, EVERTED, 0.4

4.1

10.0

$4.1 / 4.5$

VERTICAL, SLIGHT SPOOL

BURNISHED

0.6

LOW-WAISTED

13.7

12.6

BURNISHED; INCISED/TRAILED 2MM WIDE LINES, 5-8MM APART CUT INTO DRY PASTE INTERLOCKING SCROLLS ON TOP OVER 4 NESTED INVERTED ARCHES

CIRCULAR/FLAT

5.5

Associated Funerary Object; Huddleston Burial 16, 12/31/1940; Social Hill - Deceiper phase 
JEC Hodges Collection, 77-1

Artifact No. / Old Cat. No. / Site No.

Vessel Form

ARAS/HSU Digital Photo

Type

Decoration

Rim :: Body

Paste

Temper

Core Color (Munsell)

Exterior Color (Munsell)

Interior Color (Munsell)

Vessel Overall Height (cm)

Vessel Overall Weight (g)

Vessel Maximum Diameter $(\mathrm{cm})$

Volume (liters)

Usewear/Sooting/Condition

Shape/Description

Lip Treatment/Shape/Angle/Thickness

Orifice Exterior Diameter $(\mathrm{cm})$

Rim/Neck Height (cm)

Neck Mid/Base Exterior Diameter $(\mathrm{cm})$

Rim/Neck Shape/Angle

Rim/Neck Surface/Decorative Treatment

Rim/Neck/Body Thickness (cm)

Body Shape

Body Maximum Diameter $(\mathrm{cm})$

Body Height (cm)

Body Surface/Decorative Treatment

Base Shape/Curvature

Base Diameter/Height (cm)

Base Surface/Decorative Treatment

Appendages/Handles

Notes
2-14 / 2255 / 3HS 19

bottle

1296

plain/red ochre

HARD, COMPACT, MICA IN PASTE

SHELL (LEACHED)

BLACK (10YR2/1), DARK GRAY (10YR4/1), RED (10R5/8) OCHRE

GRAY (10YR5/1)

13.0

246

11.0

LIGHT WEAR ON BASE; BOTTLE IS INTACT; 2255 INKED ON BASE

SMALL GLOBULAR BOTTLE ROUNDED BASE-SHORT NECK WITH OUTFLARED RIMPLAIN-LOOKS LIKE BODY RUBBED WITH RED OCHRE RATHER THAN APPLIED SLIP

ROUNDED, EVERTED/FLARED

5.0

3.8

SPOOL

SMOOTHED \& BURNISHED

0.5

GLOBULAR

11.0

9.2

SMOOTHED \& BURNISHED; PLAIN-RED OCHRE APPLIED TO BODY-LOOKS RUBBED ON RATHER THAN APPLIED AS SLIP

UNDISTINGUISHED/CONVEX

Huddleston Burial 14, 6/1/1940; Deceiper phase 


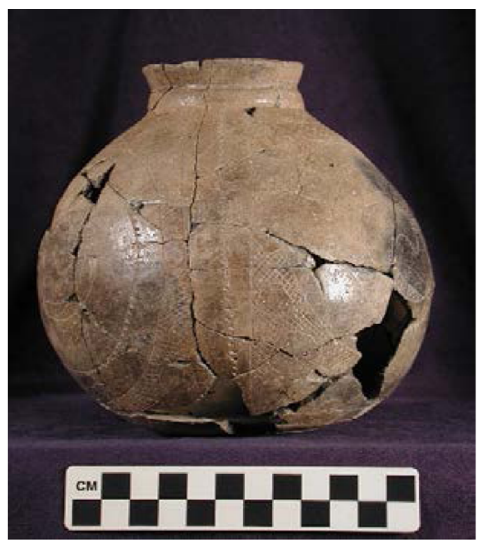

Artifact No. / Old Cat. No. / Site No.

Vessel Form

ARAS/HSU Digital Photo

Type

Decoration

$\operatorname{Rim}::$ Body

Paste

Temper

Core Color (Munsell)

Exterior Color (Munsell)

Interior Color (Munsell)

Vessel Overall Height $(\mathrm{cm})$

Vessel Overall Weight (g)

Vessel Maximum Diameter $(\mathrm{cm})$

Volume (liters)

Usewear/Sooting/Condition

Shape/Description

Lip Treatment/Shape/Angle/Thickness

Orifice Exterior Diameter $(\mathrm{cm})$

Rim/Neck Height (cm)

Neck Mid/Base Exterior Diameter $(\mathrm{cm})$

Rim/Neck Shape/Angle

Rim/Neck Surface/Decorative Treatment

Rim/Neck/Body Thickness (cm)

Body Shape

Body Maximum Diameter $(\mathrm{cm})$

Body Height (cm)

Body Surface/Decorative Treatment

Base Shape/Curvature

Base Diameter/Height $(\mathrm{cm})$

Base Surface/Decorative Treatment

Appendages/Handles

Notes
2-15 / 2240 / 3HS19

bottle

3412

Hodges Engraved var. Hodges

engraved

plain :: Ebbet 14 (sim.)

HARD, SILTY, COMPACT

SHELL (PARTLY LEACHED), FINE \& ABUNDANT + GROG, FINE \& SPARSE

BLACK (10YR2/1) (CORE MODE F)

V DK GRAY (10YR3/1), PALE BROWN (10YR6/3), V DK GRAYISH BRN (10YR3/2)

YELLOWISH BROWN (10YR5/4)

16.5

418

16.0

LIGHT ROUGHENING BASE EXT; FIRECLOUDING; RECONSTRUCTED WITH GLUE AND TAPE IN PAST (REGLUED), SEV PCS MISSING; 2240 INKED ON BASE

BOTTLE; SHORT-NECK BOTTLE, SLIGHTLY FLATTENED BOTTOM, ENGRAVED

ROUNDED, SLIGHTLY EVERTED

6.3

2.0

$5.6 / 6.0$

SPOOL (SHORT)

SMOOTHED \& POLISHED

$0.5-0.4$

GLOBULAR

16.0

14.5

POLISHED; ENGRAVED (WITH RED PIGMENT IN LINES), TICKED LINES, CROSSHATCHING WITH BALLS IN INTERIOR, INTERLOCKING SCROLLS, CIRCLES; DESIGN REPEATS $3 X$ AROUND VESSEL

CIRCULAR/FLAT

7.5

Associated Funerary Object; Huddleston Burial 11, 5/11/1940; Deceiper phase 
JEC Hodges Collection, 77-1

Artifact No. / Old Cat. No. / Site No.

Vessel Form

ARAS/HSU Digital Photo

Type

Decoration

Rim :: Body

Paste

Temper

Core Color (Munsell)

Exterior Color (Munsell)

Interior Color (Munsell)

Vessel Overall Height $(\mathrm{cm})$

Vessel Overall Weight (g)

Vessel Maximum Diameter $(\mathrm{cm})$

Volume (liters)

Usewear/Sooting/Condition

Shape/Description

Lip Treatment/Shape/Angle/Thickness

Orifice Exterior Diameter $(\mathrm{cm})$

Rim/Neck Height (cm)

Neck Mid/Base Exterior Diameter $(\mathrm{cm})$

Rim/Neck Shape/Angle

Rim/Neck Surface/Decorative Treatment

Rim/Neck/Body Thickness (cm)

Body Shape

Body Maximum Diameter $(\mathrm{cm})$

Body Height $(\mathrm{cm})$

Body Surface/Decorative Treatment

Base Shape/Curvature

Base Diameter/Height $(\mathrm{cm})$

Base Surface/Decorative Treatment

Appendages/Handles

Notes
2-16 / 2394 / 3HS19

bottle

3416

Keno Trailed

incised

plain :: Belhaven 6

HARD, SILTY, COMPACT

SHELL (PARTLY LEACHED), FINE \& ABUNDANT

V DK GRAY (10YR3/1), LT YELL BRN (10YR6/4), V DK GRAYISH BRN (10YR3/2)

VERY PALE BROWN (10YR7/4)

21.8

756

12.4

0.5

FIRECLOUDING; RECONSTRUCTED WITH GLUE IN PAST, 2 CRACKS; 2394 INKED NEAR BASE

BOTTLE; LONG-NECK BOTTLE, FLAT BOTTOM, INCISED

ROUNDED, SLIGHTLY EVERTED

4.0

10.0

$4.0 / 4.2$

VERTICAL, SLIGHT SPOOL

SMOOTHED \& BURNISHED

0.6

LOW-WAISTED

12.4

11.8

SMOOTHED \& BURNISHED; INCISED, INTERLOCKING SCROLLS (4X) ABOVE NESTED INVERTED ARCHES, MED/WIDE TRAILED 2 MM LINES, 3-6MM APART

CIRCULAR/FLAT

5.1

Associated Funerary Object; Huddleston Burial 16, 12/31/1940; Social Hill - Deceiper phase 


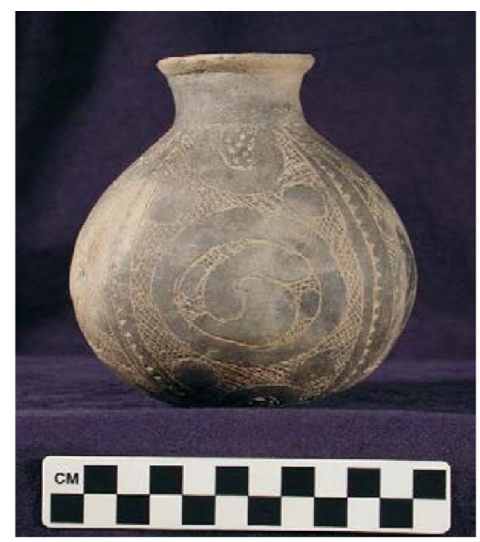

Artifact No. / Old Cat. No. / Site No.

Vessel Form

ARAS/HSU Digital Photo

Type

Decoration

Rim :: Body

Paste

Temper

Core Color (Munsell)

Exterior Color (Munsell)

Interior Color (Munsell)

Vessel Overall Height $(\mathrm{cm})$

Vessel Overall Weight (g)

Vessel Maximum Diameter $(\mathrm{cm})$

Volume (liters)

Usewear/Sooting/Condition

Shape/Description

Lip Treatment/Shape/Angle/Thickness

Orifice Exterior Diameter $(\mathrm{cm})$

Rim/Neck Height ( $\mathrm{cm}$ )

Neck Mid/Base Exterior Diameter $(\mathrm{cm})$

Rim/Neck Shape/Angle

Rim/Neck Surface/Decorative Treatment

Rim/Neck/Body Thickness (cm)

Body Shape

Body Maximum Diameter $(\mathrm{cm})$

Body Height (cm)

Body Surface/Decorative Treatment

Base Shape/Curvature

Base Diameter/Height $(\mathrm{cm})$

Base Surface/Decorative Treatment

Appendages/Handles

Notes
2-17 / $2181 / 3$ HS19

bottle

3414

Hodges Engraved var. Hodges

engraved

plain :: Ebbet 14

HARD, SILTY, COMPACT

GROG, FINE \& ABUNDANT + SHELL (LEACHED) FINE AND SPARSE

DARK GRAY (10YR4/1), VERY DARK GRAY (10YR3/1), WHITE (10YR8/1)

VERY PALE BROWN (10YR7/3)

13.0

304

11.6

BASE IS A LITTLE WORN; INTACT VESSEL, SMALL 'GASH' ABOVE ORIGINAL CATALOG NUMBER

SHORT-NECKED BOTTLE

FLATTENED, EVERTED/FLARED

5.1

2.5

CARAFE / SPOOL (SHORT)

SMOOTHED \& BURNISHED

$0.6-0.5$

GLOBULAR

11.6

7.9

ENGRAVED, RED PIGMENT IN CROSSHATCHING, 2 PATTERNS REPEATED 2X EACH, BALLS, YIN-YANG SHAPE, TICKED LINES, SWIRL, CROSSHATCHING

UNDISTINGUISHED/CONVEX

Huddleston Burial 2, 3/24/1940; Deceiper phase 
Artifact No. / Old Cat. No. / Site No.

Vessel Form

ARAS/HSU Digital Photo

Type

Decoration

Rim :: Body

Paste

Temper

Core Color (Munsell)

Exterior Color (Munsell)

Interior Color (Munsell)

Vessel Overall Height $(\mathrm{cm})$

Vessel Overall Weight (g)

Vessel Maximum Diameter $(\mathrm{cm})$

Volume (liters)

Usewear/Sooting/Condition

Shape/Description

Lip Treatment/Shape/Angle/Thickness

Orifice Exterior Diameter $(\mathrm{cm})$

Rim/Neck Height (cm)

Neck Mid/Base Exterior Diameter (cm)

Rim/Neck Shape/Angle

Rim/Neck Surface/Decorative Treatment

Rim/Neck/Body Thickness (cm)

Body Shape

Body Maximum Diameter $(\mathrm{cm})$

Body Height $(\mathrm{cm})$

Body Surface/Decorative Treatment

Base Shape/Curvature

Base Diameter/Height $(\mathrm{cm})$

Base Surface/Decorative Treatment

Appendages/Handles

Notes
2-18/2165/3HS19

bottle

3418

Hodges Engraved var. Hodges

engraved

plain :: Ebbet 14

HARD, SILTY, COMPACT

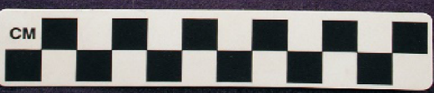

shell, leached, fine and abundant + grog, fine and abundant

DARK GRAY (10YR4/1) (CORE MODE F)

BROWN (10YR5/3), VERY DARK GRAY (10YR3/1), LT YELLOW BRN (10YR6/4)

REDDISH YELLOW (7.5YR6/6)

12.3

251

12.0

0.6

WEAR ON BASE EXTERIOR; FIRE CLOUDING; VESSEL INTACT EXCEPT FOR PROBE HOLE THROUGH SIDE; 2165 INKED ON BASE

SHORT-NECK BOTTLE, ROUNDED BASE, ENGRAVED

SMOOTHED/ERODED, FLAT, EVERTED

5.8

1.8

$5.2 / 5.5$

Spool, short

SMOOTHED \& BURNISHED/ERODED

$0.6-0.5$

Low-Waisted

12.0

10.5

SMOOTHED \& BURNISHED; ENGRAVED (RED PIGMENT TRACES), TICKED LINE, CROSSHATCHING, BALLS, REPEAT $3 \mathrm{X}$

Undistinguished/Convex

Associated Funerary Object; Huddleston Burial 1, 3/23 or 3/24/1940 (Huddleston noted this vessel with child in grave); Deceiper phase 


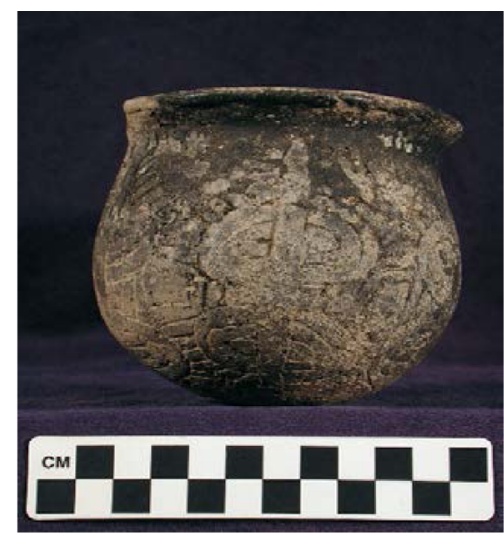

Artifact No. / Old Cat. No. / Site No.

Vessel Form

ARAS/HSU Digital Photo

Type

Decoration

Rim :: Body

Paste

Temper

Core Color (Munsell)

Exterior Color (Munsell)

Interior Color (Munsell)

Vessel Overall Height (cm)

Vessel Overall Weight (g)

Vessel Maximum Diameter $(\mathrm{cm})$

Volume (liters)

Usewear/Sooting/Condition

Shape/Description

Lip Treatment/Shape/Angle/Thickness

Orifice Exterior Diameter $(\mathrm{cm})$

Rim/Neck Height (cm)

Neck Mid/Base Exterior Diameter $(\mathrm{cm})$

Rim/Neck Shape/Angle

Rim/Neck Surface/Decorative Treatment

Rim/Neck/Body Thickness (cm)

Body Shape

Body Maximum Diameter (cm)

Body Height $(\mathrm{cm})$

Body Surface/Decorative Treatment

Base Shape/Curvature

Base Diameter/Height (cm)

Base Surface/Decorative Treatment

Appendages/Handles

Notes
2-19 / 2168 / 3HS19

jar, short rim

3392, N23018

incised

plain :: Berry 3 or 11 (sim.)

HARD, SMOOTH, COMPACT

SHELL (PARTLY LEACHED), COARSE \& ABUNDANT

VERY PALE BROWN (10YR7/4), VERY DARK GRAY (10YR3/1) (CORE MODE G)

BLACK (10YR2/1), VERY DARK GRAY (10YR3/1), BROWN (10YR5/3)

BLACK (10YR2/1), DARK GRAY (10YR4/1), YELLOWISH BROWN (10YR5/4)

9.8

249

10.9

0.5

SPALLING ON UPPER BODY INT, SURFACE ERODED; HEAVY SOOT/RESIDUE ON INT/EXT RIM/UPPER BODY; PIECE GLUED ON RIM IN PAST, ABOUT 1/3 RIM MISSING; 2168 INKED ON BASE

SMALL SHORT-RIMMED JAR OR FLARED RIM BOWL, ROUNDED BASE

ROUNDED, NONE, 0.5

10.4

1.0

CONCAVE, OUTSLANTED

SMOOTHED

$0.6-0.5$

GLOBULAR

10.9

8.8

SMOOTHED; INCISED, DRY PASTE, NESTED ARCS WITH LINES PROJECTING FROM THE ARCS, VERTICAL LINES, REPEATS 5X

UNDISTINGUISHED/CONVEX

Associated Funerary Object; Huddleston Burial 1, 3/23 or 3/24/1940 (Huddleston noted this vessel with adult in grave); Deceiper phase 
Artifact No. / Old Cat. No. / Site No.

Vessel Form

ARAS/HSU Digital Photo

Type

Decoration

Rim :: Body

Paste

Temper

Core Color (Munsell)

Exterior Color (Munsell)

Interior Color (Munsell)

Vessel Overall Height (cm)

Vessel Overall Weight (g)

Vessel Maximum Diameter $(\mathrm{cm})$

Volume (liters)

Usewear/Sooting/Condition

Shape/Description

Lip Treatment/Shape/Angle/Thickness

Orifice Exterior Diameter $(\mathrm{cm})$

Rim/Neck Height (cm)

Neck Mid/Base Exterior Diameter $(\mathrm{cm})$

Rim/Neck Shape/Angle

Rim/Neck Surface/Decorative Treatment

Rim/Neck/Body Thickness (cm)

Body Shape

Body Maximum Diameter $(\mathrm{cm})$

Body Height $(\mathrm{cm})$

Body Surface/Decorative Treatment

Base Shape/Curvature

Base Diameter/Height (cm)

Base Surface/Decorative Treatment

Appendages/Handles

Notes
2-20 / 2489 / 3HS 19

bowl, simple

3424

engraved

Erie $6::$ plain

HARD, SILTY, COMPACT

GROG, FINE \& ABUNDANT + SHELL (SOME LEACHED), FINE \& SPARSE

GRAY (10YR6/1)

V DK GRAY (10YR3/1), V PALE BROWN (10YR7/4), GRAYISH BROWN (10YR5/2)

VERY DARK GRAY (10YR3/1), VERY PALE BROWN (10YR7/4)

9.0

616

19.0

BOTTOM IS SLIGHTLY WORN; SMALL SOOT AREAS AROUND THE VESSEL, FIRECLOUDING; PART OF LIP CHIPPED AWAY, ONE SHERD RE-GLUED

SIMPLE BOWL

ROUNDED, EVERTED (FLARED)

19.0

3.0

STRAIGHT, OUTSLANTED (CONTINUOUS WITH BODY)

SMOOTHED \& BURNISHED, ENGRAVED, 4 PARALLEL ENGRAVED LINES AROUND RIM, $\sim 10 \mathrm{MM}$ APART, UNDULATING

0.6

CONVEX

16.6

6.0

SMOOTHED \& BURNISHED; PLAIN

UNDISTINGUISHED/CONVEX

Huddleston Burial X, 5/9/1941 (Huddleston note 'lone pot'); Social Hill phase 


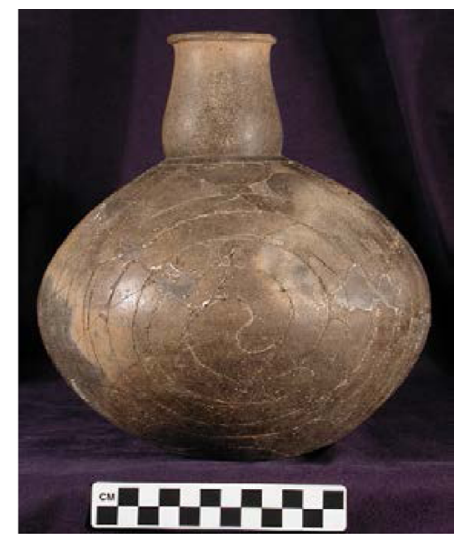

Artifact No. / Old Cat. No./ Site No.

Vessel Form

ARAS/HSU Digital Photo

Type

Decoration

Rim :: Body

Paste

Temper

Core Color (Munsell)

Exterior Color (Munsell)

Interior Color (Munsell)

Vessel Overall Height $(\mathrm{cm})$

Vessel Overall Weight (g)

Vessel Maximum Diameter $(\mathrm{cm})$

Volume (liters)

Usewear/Sooting/Condition

Shape/Description

Lip Treatment/Shape/Angle/Thickness

Orifice Exterior Diameter $(\mathrm{cm})$

Rim/Neck Height (cm)

Neck Mid/Base Exterior Diameter $(\mathrm{cm})$

Rim/Neck Shape/Angle

Rim/Neck Surface/Decorative Treatment

Rim/Neck/Body Thickness (cm)

Body Shape

Body Maximum Diameter $(\mathrm{cm})$

Body Height $(\mathrm{cm})$

Body Surface/Decorative Treatment

Base Shape/Curvature

Base Diameter/Height (cm)

Base Surface/Decorative Treatment

Appendages/Handles

Notes
2-21 / $2251 / 3$ HS19

bottle

3422

Hodges Engraved var. Hodges

engraved

plain :: Ebbet 5

HARD, SILTY, COMPACT

SHELL (SOME LEACHED), FINE \& ABUNDANT, POSS GROG, FINE \& SPARSE

STRONG BROWN (10YR5/6), YELLOW BROWN (10YR5/4), DK GRAY (10YR4/1)

LIGHT YELLOWISH BROWN (10YR6/4), DARK GRAY (10YR4/1)

25.0

865

20.7

BASE WORN SOME AROUND THE EDGE; SMALL BURNED AREAS/ FIRECLOUDING; VESSEL RECONSTRUCTED WITH GLUE AND SOME PLASTER FILLING IN PAST

MEDIUM SPOOL-NECK BOTTLE

ROUNDED, EVERTED (FLARED)

5.8

7.0

BULBOUS / SPOOL

SMOOTHED \& BURNISHED

$0.6-0.4$

GLOBULAR

20.7

18.0

SMOOTHED \& BURNISHED; ENGRAVED, CROSSHATCHING, BALLS, TICKED AND UNTICKED LINES

CIRCULAR/FLATTENED

6.7

Huddleston Burial 13, 5/19/1940; Deceiper phase 
JEC Hodges Collection, 77-1

Artifact No. / Old Cat. No. / Site No.

Vessel Form

ARAS/HSU Digital Photo

Type

Decoration

Rim :: Body

Paste

Temper

Core Color (Munsell)

Exterior Color (Munsell)

Interior Color (Munsell)

Vessel Overall Height (cm)

Vessel Overall Weight (g)

Vessel Maximum Diameter $(\mathrm{cm})$

Volume (liters)

Usewear/Sooting/Condition

Shape/Description

Lip Treatment/Shape/Angle/Thickness

Orifice Exterior Diameter $(\mathrm{cm})$

Rim/Neck Height (cm)

Neck Mid/Base Exterior Diameter $(\mathrm{cm})$

Rim/Neck Shape/Angle

Rim/Neck Surface/Decorative Treatment

Rim/Neck/Body Thickness (cm)

Body Shape

Body Maximum Diameter $(\mathrm{cm})$

Body Height $(\mathrm{cm})$

Body Surface/Decorative Treatment

Base Shape/Curvature

Base Diameter/Height $(\mathrm{cm})$

Base Surface/Decorative Treatment

Appendages/Handles

Notes
2-22 / $2260 / 3$ HS19

bowl, carinated

3420

Friendship Engraved var. Freeman

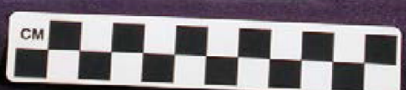

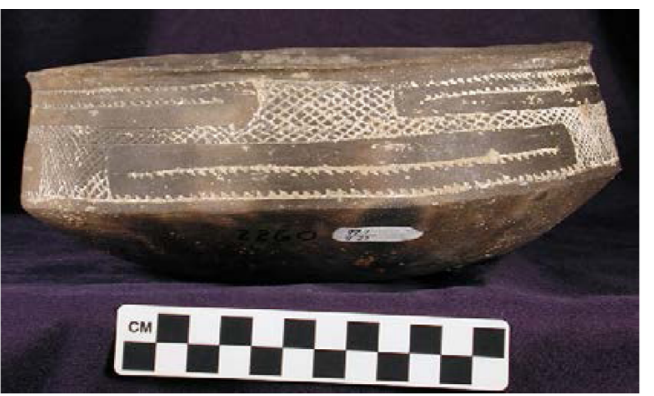

engraved

Elaine 3 :: plain

HARD, SILTY, COMPACT

SHELL, FINE AND ABUNDANT, POSS GROG, FINE \& SPARSE

GRAY (10YR6/1)

V DK GRAY (10YR3/1), DARK GRAY (10YR4/1), GRAYISH BRN (10YR5/2)

VERY DARK GRAY (10YR3/1), DARK GRAY (10YR4/1), STRONG BROWN (7.5YR5/8)

9.0

712

21.0

SCRATCHED ON BOTTOM OF INTERIOR; FIRECLOUDING; INTACT VESSEL; 2260 INKED ON BODY EXT

CARINATED BOWL, ENGRAVED

BEVELED, EVERTED (FLARED)

20.0

5.0

STRAIGHT, INSLANTED/CARINATED

SMOOTHED \& BURNISHED, ENGRAVED, 3 TIERS WITH OVALS, CROSSHATCHING, TICKED LINES, WHITE (10YR8/1) PIGMENT IN ENGRAVING

0.5

CONVEX

21.0

4.0

SMOOTHED \& BURNISHED; PLAIN

UNDISTINGUISHED/CONVEX

Huddleston Burial 15, 6/4/1940; Mid-Ouachita phase 

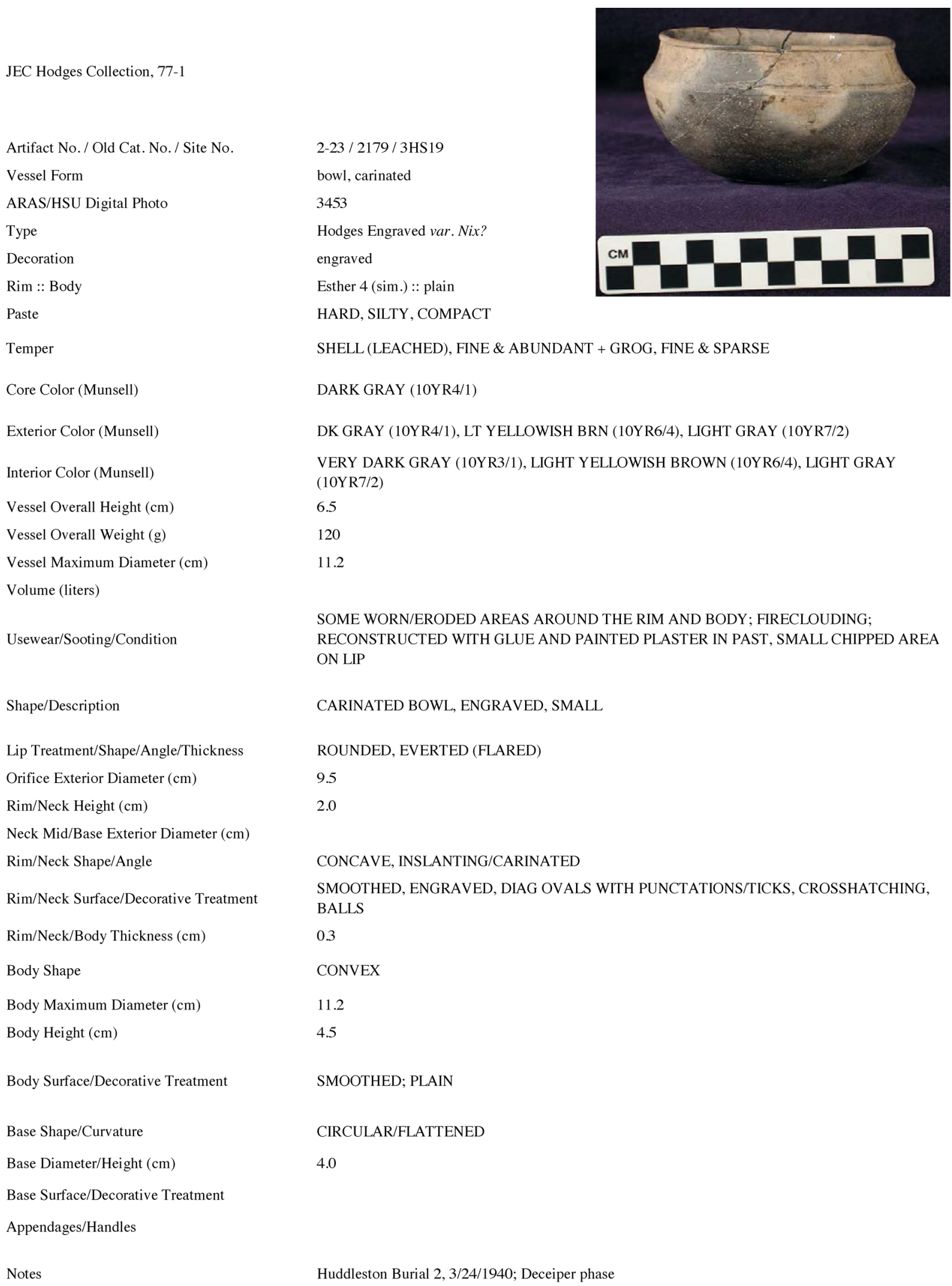
JEC Hodges Collection, 77-1

Artifact No. / Old Cat. No. / Site No.

Vessel Form

ARAS/HSU Digital Photo

Type

Decoration

Rim :: Body

Paste

Temper

Core Color (Munsell)

Exterior Color (Munsell)

Interior Color (Munsell)

Vessel Overall Height $(\mathrm{cm})$

Vessel Overall Weight (g)

Vessel Maximum Diameter $(\mathrm{cm})$

Volume (liters)

Usewear/Sooting/Condition

Shape/Description

Lip Treatment/Shape/Angle/Thickness

Orifice Exterior Diameter $(\mathrm{cm})$

Rim/Neck Height (cm)

Neck Mid/Base Exterior Diameter $(\mathrm{cm})$

Rim/Neck Shape/Angle

Rim/Neck Surface/Decorative Treatment

Rim/Neck/Body Thickness (cm)

Body Shape

Body Maximum Diameter $(\mathrm{cm})$

Body Height (cm)

Body Surface/Decorative Treatment

Base Shape/Curvature

Base Diameter/Height $(\mathrm{cm})$

Base Surface/Decorative Treatment

Appendages/Handles

Notes
2-24 / 2253 / 3HS19

bowl, carinated

593,1306

Hodges Engraved var. Nix

engraved

Esther $12::$ plain

HARD, COMPACT

SHELL, FINE \& MEDIUM ABUNDANT

BLACK, VERY PALE BROWN, RED PIGMENT IN ENGRAVING

BLACK

7.0

178

13.3

FIRECLOUDING; 2 PROBE HOLES IN BASE, VESSEL RECONSTRUCTED WITH GLUE, 2 SHERDS MISSING

CARINATED BOWL, SMALL

ROUNDED, SLIGHTLY EVERTED

12.1

2.3

STRAIGHT, INSLANTED/CARINATED

SMOOTHED \& BURNISHED, ENGRAVED, SCROLL, TICKED LINE, CROSSHATCHING, BALLS

$0.4-0.5$

CONVEX/GLOBULAR

13.3

4.7

SMOOTHED \& BURNISHED; PLAIN

UNDISTINGUISHED/CONVEX

Huddleston Burial 14, 6/1/1940; Deceiper phase 


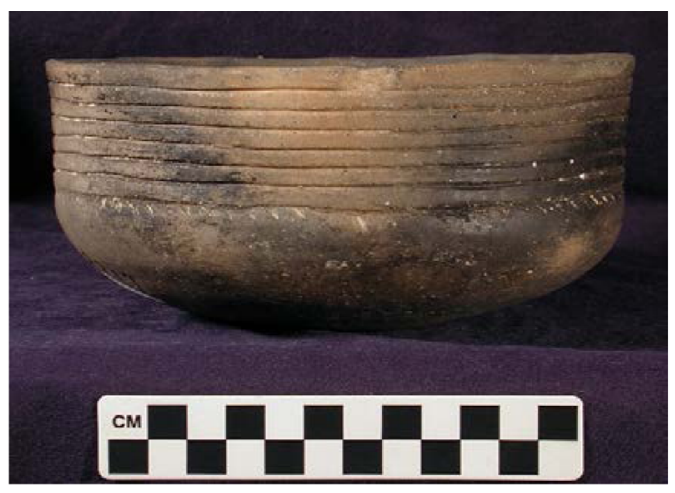

Artifact No. / Old Cat. No. / Site No.

Vessel Form

ARAS/HSU Digital Photo

Type

Decoration

Rim :: Body

Paste

Temper

Core Color (Munsell)

Exterior Color (Munsell)

Interior Color (Munsell)

Vessel Overall Height $(\mathrm{cm})$

Vessel Overall Weight (g)

Vessel Maximum Diameter $(\mathrm{cm})$

Volume (liters)

Usewear/Sooting/Condition

Shape/Description

Lip Treatment/Shape/Angle/Thickness

Orifice Exterior Diameter $(\mathrm{cm})$

Rim/Neck Height (cm)

Neck Mid/Base Exterior Diameter $(\mathrm{cm})$

Rim/Neck Shape/Angle

Rim/Neck Surface/Decorative Treatment

Rim/Neck/Body Thickness (cm)

Body Shape

Body Maximum Diameter $(\mathrm{cm})$

Body Height $(\mathrm{cm})$

Body Surface/Decorative Treatment

Base Shape/Curvature

Base Diameter/Height $(\mathrm{cm})$

Base Surface/Decorative Treatment

Appendages/Handles

Notes
2-25 / 2217 / 3HS19

bowl, simple

3455

engraved/incised

Elgin $4::$ plain

HARD, SILTY, COMPACT

GROG, FINE \& ABUNDANT + SHELL (SOME LEACHED), FINE \& SPARSE

VERY DARK GRAYISH BROWN (10YR3/2)

BLACK (10YR2/1), DARK GRAY (10YR4/1), YELLOWISH BROWN (10YR5/4)

BLACK (10YR2/1), VERY DARK GRAY (10YR3/1), LIGHT YELLOWISH BROWN (10YR6/4)

8.5

500

16.2

BOTTOM MODERATELY WORN; SOOT PATCHES/FIRECLOUDING; VESSEL INTACT EXCEPT FOR PROBE HOLE IN BOTTOM AND SMALL CHIP ON RIM; 2217 INKED ON BASE

TALL-RIM SIMPLE BOWL (STRAIGHT SIDES), ENGRAVED AND INCISED

DIRECT

16.2

4.0

STRAIGHT, VERTICAL

SMOOTHED \& BURNISHED, ENGRAVED \& INCISED, 7 HORIZ LINES (0.5CM APART), LOWEST TICKED (UPPER LINES INCISED NOT ENGRAVED?)

0.6

CONVEX

16.0

4.5

SMOOTHED \& BURNISHED; PLAIN

UNDISTINGUISHED/CONVEX

Huddleston Burial 9, 4/27/1940; Social Hill phase 
Artifact No. / Old Cat. No. / Site No.

Vessel Form

ARAS/HSU Digital Photo

Type

Decoration

$\operatorname{Rim}::$ Body

Paste

Temper

Core Color (Munsell)

Exterior Color (Munsell)

Interior Color (Munsell)

Vessel Overall Height $(\mathrm{cm})$

Vessel Overall Weight (g)

Vessel Maximum Diameter (cm)

Volume (liters)

Usewear/Sooting/Condition

Shape/Description

Lip Treatment/Shape/Angle/Thickness Orifice Exterior Diameter (cm)

Rim/Neck Height (cm)

Neck Mid/Base Exterior Diameter $(\mathrm{cm})$

Rim/Neck Shape/Angle

Rim/Neck Surface/Decorative Treatment

Rim/Neck/Body Thickness (cm)

Body Shape

Body Maximum Diameter (cm)

Body Height (cm)

Body Surface/Decorative Treatment

Base Shape/Curvature

Base Diameter/Height $(\mathrm{cm})$

Base Surface/Decorative Treatment

Appendages/Handles

Notes
2-26/2231/3HS19

bowl, simple

3461, N21658

plain

HARD, SILTY, COMPACT

SHELL (SOME LEACHED), MEDIUM SIZED \& ABUNDANT

VERY DARK GRAY (10YR3/1), GRAY (10YR5/1) (CORE MODE B)

V DK GRAY (10YR3/1), DARK GRAY (10YR4/1), LT YELLOW BROWN (10YR6/4)

DARK GRAY (10YR4/1), BLACK (10YR2/1), BROWN (10YR4/3)

9.0

461

25.5

SOME FIRECLOUDING; RECONSTRUCTED WITH GLUE IN PAST AND REGLUED, SEVERAL RIM/BODY/BASE PIECES MISSING; 2231 INKED ON EXT SIDE

SIMPLE BOWL, LARGE

ROUNDED, SLIGHTLY EVERTED

25.5

CONTINUOUS WITH BODY

STRAIGHT, OUTSLANTED

BURNISHED

$0.3-0.5$

CONICAL

9.0

SMOOTHED \& BURNISHED; PLAIN

CIRCULAR/SLIGHTLY CONCAVE (0.3CM)

8.0

Associated Funerary Object; Huddleston Burial 10, 5/5/1940; Social Hill phase 
Artifact No. / Old Cat. No. / Site No.

Vessel Form

ARAS/HSU Digital Photo

Type

Decoration

Rim :: Body

Paste

Temper

Core Color (Munsell)

Exterior Color (Munsell)

Interior Color (Munsell)

Vessel Overall Height $(\mathrm{cm})$

Vessel Overall Weight (g)

Vessel Maximum Diameter $(\mathrm{cm})$

Volume (liters)

Usewear/Sooting/Condition

Shape/Description

Lip Treatment/Shape/Angle/Thickness

Orifice Exterior Diameter $(\mathrm{cm})$

Rim/Neck Height (cm)

Neck Mid/Base Exterior Diameter $(\mathrm{cm})$

Rim/Neck Shape/Angle

Rim/Neck Surface/Decorative Treatment

Rim/Neck/Body Thickness (cm)

Body Shape

Body Maximum Diameter $(\mathrm{cm})$

Body Height $(\mathrm{cm})$

Body Surface/Decorative Treatment

Base Shape/Curvature

Base Diameter/Height $(\mathrm{cm})$

Base Surface/Decorative Treatment

Appendages/Handles

Notes
2-27 / 2241 / 3HS19

bowl, carinated

1122, N22787

Hodges Engraved var. Hodges

engraved

Esther 11 (type) :: Ebbet 13 (sim.)

HARD, SMOOTH, COMPACT

SHELL (LEACHED), FINE \& ABUNDANT + GROG, FINE \& SPARSE

VERY DARK GRAY (10YR3/1) (CORE MODE F)

BROWN (10YR4/3), STRONG BROWN (7.5YR5/6), BLACK (10YR2/1)

DARK GRAYISH BROWN (10YR4/2)

8.2

250

16.0

FIRECLOUDING; VESSEL RECONSTRUCTED WITH GLUE AND PLASTER IN PAST, LIP CHIPPED, PROBE? HOLE IN BODY, MUCH OF BASE MISSING; 2241 INKED ON BASE

CARINATED BOWL, ENGRAVED, ROUNDED BASE-TRACE OF RED PIGMENT IN ENGRAVED LINES

THICKENED SLIGHTLY, SLIGHTLY EVERTED

15.5

2.8

STRAIGHT, CARINATED

BURNISHED, ENGRAVED, CROSSHATCHING, BALLS, TICKED LINE SCROLLS, REPEATS $4 \mathrm{X}$

$0.4-0.3$

CONVEX

16.0

5.4

SMOOTHED \& BURNISHED; ENGRAVED, TICKED LINES, HOOKED ELEMENTS, CROSSHATCHING, BALLS, REPEATS $3 \mathrm{X}$

UNDISTINGUISHED/CONVEX

Associated Funerary Object; Huddleston Burial 11, 5/11/1940; Deceiper phase 
Artifact No. / Old Cat. No. / Site No.

Vessel Form

ARAS/HSU Digital Photo

Type

Decoration

$\operatorname{Rim}::$ Body

Paste

Temper

Core Color (Munsell)

Exterior Color (Munsell)

Interior Color (Munsell)

Vessel Overall Height (cm)

Vessel Overall Weight (g)

Vessel Maximum Diameter (cm)

Volume (liters)

Usewear/Sooting/Condition

Shape/Description

Lip Treatment/Shape/Angle/Thickness

Orifice Exterior Diameter (cm)

Rim/Neck Height (cm)

Neck Mid/Base Exterior Diameter (cm)

Rim/Neck Shape/Angle

Rim/Neck Surface/Decorative Treatment

Rim/Neck/Body Thickness (cm)

Body Shape

Body Maximum Diameter $(\mathrm{cm})$

Body Height $(\mathrm{cm})$

Body Surface/Decorative Treatment

Base Shape/Curvature

Base Diameter/Height (cm)

Base Surface/Decorative Treatment

Appendages/Handles

Notes
2-29 / 2177 / 3HS19

jar, short rim

3463, N22718

incised

Austin? :: Buffalo 12 (sim.)

SOFT, GRANULAR, CRUMBLY

SHELL (SOME LEACHED), COARSE \& ABUNDANT

GRAY (10YR6/1) (CORE MODE B)

BLACK (10YR2/1), VERY DARK GRAY (10YR3/1), DARK GRAY (10YR4/1)

LIGHT YELLOWISH BROWN (10YR6/4), BLACK (10YR2/1), DARK GRAY (10YR4/1)

9.5

228

11.5

0.6

ERODED; HEAVY SOOT RESIDUE ON BODY EXT, SOME BLACKENING ON INT RIM, FIRECLOUDING; CHIPPED LIP \& HOLE IN BODY (FROM PROBE?); 2177 INKED UPPER BODY

SMALL, SHORT RIMMED JAR, FLARED LIP, GLOBULAR BODY AND ROUNDED BASE

ROUNDED, EVERTED, 0.3

11.5

2.0

CONCAVE, VERTICAL

SMOOTHED, VERTICAL PARALLEL INCISED LINES 0.7CM APART AROUND RIM

0.4

GLOBULAR

11.3

7.5

SMOOTHED; INCISED, ROUNDED HOOK ELEMENTS, PATTERN REPEATS 5X; DRY PASTE INCISING

UNDISTINGUISHED/CONVEX

Associated Funerary Object; Huddleston Burial 1,3/23 or 3/24/1940 (Huddleston noted this vessel with child in grave); Deceiper phase 
Artifact No. / Old Cat. No. / Site No.

Vessel Form

ARAS/HSU Digital Photo

Type

Decoration

Rim :: Body

Paste

Temper

Core Color (Munsell)

Exterior Color (Munsell)

Interior Color (Munsell)

Vessel Overall Height (cm)

Vessel Overall Weight (g)

Vessel Maximum Diameter (cm)

Volume (liters)

Usewear/Sooting/Condition

Shape/Description

Lip Treatment/Shape/Angle/Thickness

Orifice Exterior Diameter (cm)

Rim/Neck Height (cm)

Neck Mid/Base Exterior Diameter (cm)

Rim/Neck Shape/Angle

Rim/Neck Surface/Decorative Treatment

Rim/Neck/Body Thickness (cm)

Body Shape

Body Maximum Diameter (cm)

Body Height (cm)

Body Surface/Decorative Treatment

Base Shape/Curvature

Base Diameter/Height (cm)

Base Surface/Decorative Treatment

Appendages/Handles

Notes
$2-31 / 2167 / 3 H S 19$

bottle

3465, N22847

Bailey Engraved

engraved

plain :: Eugene 3

HARD, SILTY, COMPACT, MICA IN PASTE

SHELL (SOME LEACHED), FINE \& ABUNDANT; OCCASIONAL PEBBLE

VERY DARK GRAY (10YR3/1) (CORE MODE F)

BLACK (10YR2/1), DARK GRAY (10YR4/1), YELLOWISH BROWN (10YR5/4)

BROWN (10YR5/3), LIGHT YELLOWISH BROWN (10YR6/4), VERY DARK GRAY (10YR3/1)

17.3

415

14.2

1.0

BASE SLIGHTLY WORN; NECK PARTIALLY RECONSTRUCTED IN PAST, LIP HEAVILY CHIPPED; 2167 INKED ON BOTTOM OF BODY

FLARED-RIM BOTTLE WITH LARGE PEDESTAL BASE AND SPOOL-SHAPED NECK

ROUNDED, EVERTED, 0.3

5.6

5.6

$5.8 / 5.7$

BULBOUS / SPOOL

SMOOTHED \& BURNISHED

0.4

LOW-WAISTED

14.2

8.7

SMOOTHED \& BURNISHED; ENGRAVED, 5 LINES UNDER NECK, 4 PANELS, U-SHAPED DESIGN WITH LINES BETWEEN, REPEATS 4X

CIRCULAR/FLAT, PEDESTAL

$6.0-9.7 / 3.1$

Associated Funerary Object; Huddleston Burial 1, 3/23 or 3/24/1940 (Huddleston noted this vessel with adult in grave); Deceiper phase 
Artifact No. / Old Cat. No. / Site No.

Vessel Form

ARAS/HSU Digital Photo

Type

Decoration

Rim :: Body

Paste

Temper

Core Color (Munsell)

Exterior Color (Munsell)

Interior Color (Munsell)

Vessel Overall Height (cm)

Vessel Overall Weight (g)

Vessel Maximum Diameter (cm)

Volume (liters)

Usewear/Sooting/Condition

Shape/Description

Lip Treatment/Shape/Angle/Thickness

Orifice Exterior Diameter (cm)

Rim/Neck Height (cm)

Neck Mid/Base Exterior Diameter (cm)

Rim/Neck Shape/Angle

Rim/Neck Surface/Decorative Treatment

Rim/Neck/Body Thickness (cm)

Body Shape

Body Maximum Diameter $(\mathrm{cm})$

Body Height $(\mathrm{cm})$

Body Surface/Decorative Treatment

Base Shape/Curvature

Base Diameter/Height (cm)

Base Surface/Decorative Treatment

Appendages/Handles

Notes
$2-32 / 2201 / 3 H S 19$

jar, tall rim

3467

Foster/Pease?

incised

Alfred $2::$ Antioch 18 (sim.)

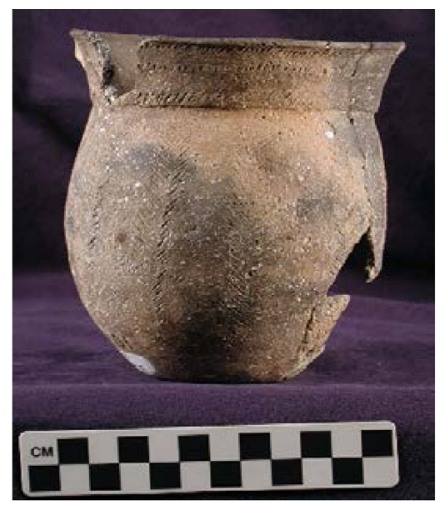

SOFT, GRANULAR, CRUMBLY

SHELL (SOME LEACHED), COARSE \& ABUNDANT

DARK GRAY (10YR4/1)

LT YELL BRN (10YR6/4), V DK GRAY (10YR3/1), DK GRAYISH BRN (10YR4/2)

VERY DARK GRAY (10YR3/1), DARK GRAY (10YR4/1), GRAY (10YR5/1)

13.5

291

11.9

VESSEL SLIGHTLY WORN/ERODED; FIRECLOUDING; PREVIOUSLY RECONSTRUCTED WITH GLUE, SECTIONS OF BODY AND RIM MISSING; 2201 INKED LOWER BODY

JAR, TALL RIM, INCISED

ROUNDED, SLIGHTLY EVERTED (FLARED)

11.9

2.5

\section{CONCAVE/OUTSLANTED}

INCISED, 3 BANDS OF DIAGONAL SLASH INCISING; SIM. TO FOSTER RIM DECORATION

0.4

ELONGATED

11.4

11.0

INCISED, VERTICAL HERRINGBONE PATTERN 13X (10 HERRINGBONE DESIGN AND 3 WITH BOTH SETS OF LINES SLANTING DOWNWARDS); SIM. TO PEASE B-I OR CANEY PUNCT. BODY DECORATION

CIRCULAR/FLATTENED

5.0

Huddleston Burial 5, 4/9/1940; Social Hill phase 
Artifact No. / Old Cat. No. / Site No.

Vessel Form

ARAS/HSU Digital Photo

Type

Decoration

Rim :: Body

Paste

Temper

Core Color (Munsell)

Exterior Color (Munsell)

Interior Color (Munsell)

Vessel Overall Height $(\mathrm{cm})$

Vessel Overall Weight (g)

Vessel Maximum Diameter $(\mathrm{cm})$

Volume (liters)

Usewear/Sooting/Condition

Shape/Description

Lip Treatment/Shape/Angle/Thickness

Orifice Exterior Diameter $(\mathrm{cm})$

Rim/Neck Height (cm)

Neck Mid/Base Exterior Diameter (cm)

Rim/Neck Shape/Angle

Rim/Neck Surface/Decorative Treatment

Rim/Neck/Body Thickness (cm)

Body Shape

Body Maximum Diameter (cm)

Body Height $(\mathrm{cm})$

Body Surface/Decorative Treatment

Base Shape/Curvature

Base Diameter/Height (cm)

Base Surface/Decorative Treatment

Appendages/Handles

Notes
2-33 / 2172 / 3HS19

bowl, simple

$598, \mathrm{~N} 21625$

plain

HARD, GRITTY, COMPACT, SAND IN PASTE

GROG, FINE \& SPARSE

LIGHT REDDISH BROWN (5YR6/3) (CORE MODE A)

YELLOWISH RED (5YR5/6), BLACK (5YR2.5/1) FIRECLOUDS

BLACK (5YR2.5/1), YELLOWISH RED (5YR5/6)

4.1

93

7.9

0.1

red ochre on int body from use?; fireclouding, black spots post-deposition; large chip to lip; 2172 inked near base

MINI-BOWL WITH CONVEX ROUND BASE, WIDE ROUNDED OVER LIP AND SLIGHTLY OUTSLANTING SIDES

SMOOTHED, ROLLED, SLIGHTLY EVERTED

7.9

Straight, Outslanted

SMOOTHED, RED OCHRE OR FIRING RATHER THAN SLIP

0.7

CONVEX

7.6

4.1

SMOOTHED; RED OCHRE OR FIRING RATHER THAN SLIP; VESSEL HAS WIPE MARKS ON INTERIOR

Undistinguished/Convex

Associated Funerary Object; Huddleston Burial 1,3/23 or 3/24/1940 (Huddleston noted this vessel with child in grave); Deceiper phase 
JEC Hodges Collection, 77-1

Artifact No. / Old Cat. No. / Site No.

Vessel Form

ARAS/HSU Digital Photo

Type

Decoration

Rim :: Body

Paste

Temper

Core Color (Munsell)

Exterior Color (Munsell)

Interior Color (Munsell)

Vessel Overall Height (cm)

Vessel Overall Weight (g)

Vessel Maximum Diameter (cm)

Volume (liters)

Usewear/Sooting/Condition

Shape/Description

Lip Treatment/Shape/Angle/Thickness Orifice Exterior Diameter $(\mathrm{cm})$

Rim/Neck Height (cm)

Neck Mid/Base Exterior Diameter (cm)

Rim/Neck Shape/Angle

Rim/Neck Surface/Decorative Treatment

Rim/Neck/Body Thickness (cm)

Body Shape

Body Maximum Diameter $(\mathrm{cm})$

Body Height $(\mathrm{cm})$

Body Surface/Decorative Treatment

Base Shape/Curvature

Base Diameter/Height (cm)

Base Surface/Decorative Treatment

Appendages/Handles

Notes
2-34 / 2185 / 3HS19

jar (sherd)

3482

Karnack Brushed-Incised var. Fish Bayou

brushed/trailed

Dana 4 :: Abraham 1

HARD, SILTY, COMPACT

SHELL, COARSE \& ABUNDANT

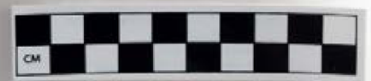

LIGHT YELLOWISH BROWN (10YR6/4), CORE MODE B

V PALE BROWN (10YR7/3), BLACK (10YR2/1), V DK GRAYISH BRN (10YR3/2)

YELLOWISH BROWN (10YR5/4), VERY DARK GRAY (10YR3/1), BLACK (10YR2/1)

13.0 (PARTIAL)

199

SOOT/RESIDUE AROUND RIM INT \& EXT UNDER RIM; SHERD ONLY OF MUCH LARGER VESSEL ; 2185 INKED ON EXT

LARGE JAR RIM FRAGMENT (SHERD)

ROUNDED, SLIGHTLY EVERTED, 0.5

30.0 (est., $15 \%$ of rim)

2.0

STRAIGHT, OUTSLANTED

BRUSHED, HORIZONTAL BRUSHING

$0.7-0.3$

GLOBULAR JAR WITH SHORT, FLARED RIM

SMOOTHED \& INCISED/TRAILED, BROAD TRAILED VERTICAL LINES, 3-4MM WIDE, 7 10MM APART

Huddleston Burial 3, 3/24/1940; Deceiper phase 
JEC Hodges Collection, 77-1

Artifact No. / Old Cat. No. / Site No.

Vessel Form

ARAS/HSU Digital Photo

Type

Decoration

Rim :: Body

Paste

Temper

Core Color (Munsell)

Exterior Color (Munsell)

Interior Color (Munsell)

Vessel Overall Height $(\mathrm{cm})$

Vessel Overall Weight $(\mathrm{g})$

Vessel Maximum Diameter (cm)

Volume (liters)

Usewear/Sooting/Condition

Shape/Description

Lip Treatment/Shape/Angle/Thickness

Orifice Exterior Diameter $(\mathrm{cm})$

Rim/Neck Height (cm)

Neck Mid/Base Exterior Diameter $(\mathrm{cm})$

Rim/Neck Shape/Angle

Rim/Neck Surface/Decorative Treatment

Rim/Neck/Body Thickness (cm)

Body Shape

Body Maximum Diameter (cm)

Body Height $(\mathrm{cm})$

Body Surface/Decorative Treatment

Base Shape/Curvature

Base Diameter/Height $(\mathrm{cm})$

Base Surface/Decorative Treatment

Appendages/Handles

Notes
$2-35 / 2182 / 3 \mathrm{HS} 19$

bottle

3488

Hodges Engraved var. Hodges

engraved

plain :: Ebbet 5 (sim.)

HARD, SILTY, COMPACT

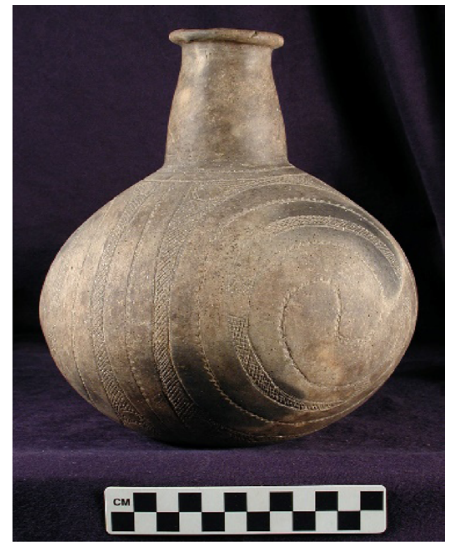

SHELL (SOME LEACHED), FINE \& ABUNDANT + GROG, FINE \& SPARSE

BLACK (10YR2/1), DARK GRAY (10YR4/1), BROWN (10YR5/3)

DARK GRAY (10YR4/1), LIGHT YELLOWISH BROWN (10YR6/4), BROWN (10YR5/3)

22.5

730

18.4

FIRECLOUDING; VESSEL INTACT WITH SMALL CHIP ON LIP

MEDIUM-NECKED GLOBULAR BOTTLE WITH SPOOL NECK AND FLARED LIP

ROUNDED, EVERTED (FLARED)

5.5

7.3

BULBOUS / SPOOL

SMOOTHED \& BURNISHED

$0.4-0.3$

GLOBULAR

18.4

15.2

SMOOTHED \& BURNISHED; ENGRAVED, DOUBLE-HOOK DESIGN, TICKED LINES, CROSSHATCHING, BALLS, REPEATS 3X W VARIATIONS

CIRCULAR, FLATTENED

6.0

Huddleston Burial 3, 3/24/1940; Deceiper phase 
JEC Hodges Collection, 77-1

Artifact No. / Old Cat. No. / Site No.

Vessel Form

ARAS/HSU Digital Photo

Type

Decoration

Rim :: Body

Paste

Temper

Core Color (Munsell)

Exterior Color (Munsell)

Interior Color (Munsell)

Vessel Overall Height (cm)

Vessel Overall Weight ( $\mathrm{g}$ )

Vessel Maximum Diameter (cm)

Volume (liters)

Usewear/Sooting/Condition

Shape/Description

Lip Treatment/Shape/Angle/Thickness Orifice Exterior Diameter (cm)

Rim/Neck Height (cm)

Neck Mid/Base Exterior Diameter (cm)

Rim/Neck Shape/Angle

Rim/Neck Surface/Decorative Treatment

Rim/Neck/Body Thickness (cm)

Body Shape

Body Maximum Diameter (cm)

Body Height $(\mathrm{cm})$

Body Surface/Decorative Treatment

Base Shape/Curvature

Base Diameter/Height (cm)

Base Surface/Decorative Treatment

Appendages/Handles

Notes
$2-36 / 2259 / 3$ HS 19

seed jar

3494

engraved

Erie 6 :: Emerson 23

HARD, SILTY, COMPACT

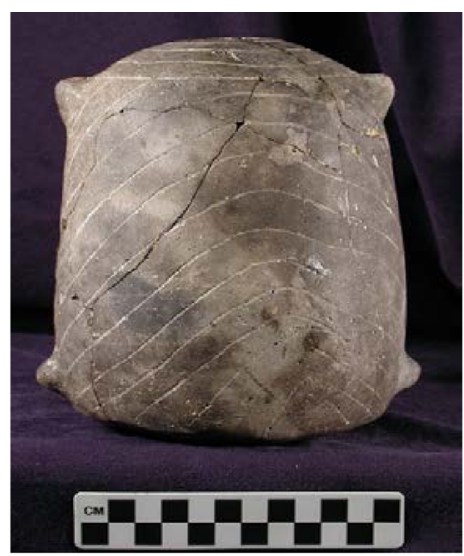

GROG, FINE \& ABUNDANT + SHELL (SOME LEACHED), FINE \& MED ABUNDANT

BLACK (10YR2/1), DARK GRAY (10YR4/1), GRAY (10YR6/1)

18.0

698

13.8

FIRECLOUDING; VESSEL RECONSTRUCTED WITH GLUE IN PAST, SMALL FRAGMENTS MISSING

LARGE SEED JAR, ENGRAVED, WITH 4 APPLIQUE NODE HANDLES WITH PERFORATIONS

FLATTENED

2.7

1.6

CONVEX, INSLANTED

SMOOTHED, ENGRAVED, 4 CONCENTRIC CIRCLES AROUND ORIFICE

0.4

BARREL

13.8

16.4

SMOOTHED; ENGRAVED, V-SHAPED LINES ENCIRCLING VESSEL

CIRCULAR, SLIGHTLY FLATTENED

12.5

4 NODE HANDLES (2 ON EITHER SIDE) WITH VERTICAL PERFORATIONS

Huddleston Burial 15, 6/4/1940; Mid-Ouachita phase 
Artifact No. / Old Cat. No. / Site No.

Vessel Form

ARAS/HSU Digital Photo

Type

Decoration

Rim :: Body

Paste

Temper

Core Color (Munsell)

Exterior Color (Munsell)

Interior Color (Munsell)

Vessel Overall Height $(\mathrm{cm})$

Vessel Overall Weight (g)

Vessel Maximum Diameter (cm)

Volume (liters)

Usewear/Sooting/Condition

Shape/Description

Lip Treatment/Shape/Angle/Thickness

Orifice Exterior Diameter (cm)

Rim/Neck Height (cm)

Neck Mid/Base Exterior Diameter (cm)

Rim/Neck Shape/Angle

Rim/Neck Surface/Decorative Treatment

Rim/Neck/Body Thickness (cm)

Body Shape

Body Maximum Diameter (cm)

Body Height $(\mathrm{cm})$

Body Surface/Decorative Treatment

Base Shape/Curvature

Base Diameter/Height (cm)

Base Surface/Decorative Treatment

Appendages/Handles

Notes
2-37 / 2233 / 3 HS 19

jar, tall rim

3492, N22646

Foster Trailed-Incised var. Foster

incised/appliqued nodes

Alfred 6 :: Baker 16 (type)

HARD, SILTY, COMPACT

SHELL, COARSE AND ABUNDANT

DARK GRAY (10YR4/1) (CORE MODE F)

PALE BROWN (10YR6/3), V DK GRAYISH BROWN (10YR3/2), BLACK (10YR2/1)

DARK GRAY (10YR4/1), GRAY (10YR5/1), PALE BROWN (10YR6/3)

11.0

297

10.7

0.5

TRACE SOOT ON EXT RIM AND BODY, FIRECLOUDING; RIM RECONSTRUCTED WITH GLUE IN PAST, SEVERAL FRAGMENTS FROM RIM/LIP MISSING; 2233 INKED ON BASE

JAR, TALL RIM GLOBULAR, INCISED WITH 3 APPLIQUE NODES AROUND VESSEL BODY

FLAT, EVERTED, 0.3

10.7

4.0

CONCAVE, VERTICAL

SMOOTHED, INCISED, 5 BANDS OF DIAGONAL SLASH INCISIONS, SPACED ROWS

$0.4-0.5$

SUBGLOBULAR

10.6

7.0

SMOOTHED; INCISED, CONCENTRIC CIRCLES, DESIGN STOPS ABOUT 1CM ABOVE BASE, 3 APPLIQUE NODES

CIRCULAR/FLAT

5.0

3 APPLIQUE NODES (2 PT CLUSTERS) ON BODY

Associated Funerary Object; Huddleston Burial 10, 5/5/1940; Social Hill phase 
JEC Hodges Collection, 77-1

Artifact No. / Old Cat. No. / Site No.

Vessel Form

ARAS/HSU Digital Photo

Type

Decoration

$\operatorname{Rim}::$ Body

Paste

Temper

Core Color (Munsell)

Exterior Color (Munsell)

Interior Color (Munsell)

Vessel Overall Height $(\mathrm{cm})$

Vessel Overall Weight (g)

Vessel Maximum Diameter (cm)

Volume (liters)

Usewear/Sooting/Condition

Shape/Description

Lip Treatment/Shape/Angle/Thickness

Orifice Exterior Diameter $(\mathrm{cm})$

Rim/Neck Height (cm)

Neck Mid/Base Exterior Diameter (cm)

Rim/Neck Shape/Angle

Rim/Neck Surface/Decorative Treatment

Rim/Neck/Body Thickness (cm)

Body Shape

Body Maximum Diameter $(\mathrm{cm})$

Body Height (cm)

Body Surface/Decorative Treatment

Base Shape/Curvature

Base Diameter/Height $(\mathrm{cm})$

Base Surface/Decorative Treatment

Appendages/Handles

Notes
$2-38 / 2243$ / 3HS19

bottle

3496, N22817

plain

HARD, SILTY, COMPACT

SHELL (SOME LEACHED), MED TO COARSE \& ABUNDANT

VERY DARK GRAY (10YR3/1) (CORE MODE F)

V DK GRAY (10YR3/1), BLACK (10YR2/1), DK GRAYISH BROWN (10YR4/2)

DARK GRAYISH BROWN (10YR4/2), BROWN (10YR5/3)

17.2

456

12.0

FIRECLOUDING; VESSEL RECONSTRUCTED WITH GLUE IN PAST, SEVERAL PIECES MISSING FROM BODY, CHIPPED LIP; 2243 INKED NEAR BASE

BOTTLE; LONG-NECK BOTTLE WITH GLOBULAR (SQUAT) BODY, FLARED LIP, AND FLAT BOTTOM.

SMOOTHED, FLAT, EVERTED, 0.3

3.9

7.5

$4.0 / 4.0$

BULBOUS / SPOOL

SMOOTHED \& BURNISHED

$0.4-0.6$

SUBGLOBULAR

12.0

9.7

SMOOTHED \& BURNISHED; PLAIN

CIRCULAR/FLAT

5.3

Associated Funerary Object; Huddleston Burial 12, 5/11/1940; Social Hill - Deceiper phase 
JEC Hodges Collection, 77-1

Artifact No. / Old Cat. No. / Site No.

Vessel Form

ARAS/HSU Digital Photo

Type

Decoration

Rim :: Body

Paste

Temper

Core Color (Munsell)

Exterior Color (Munsell)

Interior Color (Munsell)

Vessel Overall Height $(\mathrm{cm})$

Vessel Overall Weight (g)

Vessel Maximum Diameter $(\mathrm{cm})$

Volume (liters)

Usewear/Sooting/Condition

Shape/Description

Lip Treatment/Shape/Angle/Thickness

Orifice Exterior Diameter $(\mathrm{cm})$

Rim/Neck Height (cm)

Neck Mid/Base Exterior Diameter $(\mathrm{cm})$

Rim/Neck Shape/Angle

Rim/Neck Surface/Decorative Treatment

Rim/Neck/Body Thickness (cm)

Body Shape

Body Maximum Diameter (cm)

Body Height $(\mathrm{cm})$

Body Surface/Decorative Treatment

Base Shape/Curvature

Base Diameter/Height ( $\mathrm{cm})$

Base Surface/Decorative Treatment

Appendages/Handles

Notes

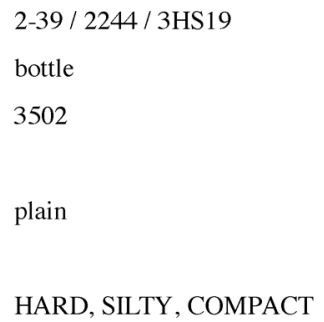

21.5

3.1

FIRECLOUDING; VESSEL RECONSTRUCTED WITH GLUE AND PLASTER IN PAST, MISSING SEVERAL PCS BODY; 2244 INKED ON SIDE OF VESSEL

BOTTLE; LARGE SHORT-NECK GLOBULAR BOTTLE WITH FLAT BASE.

FLAT, EVERTED

4.4

2.0

$4.1 / 4.5$

CARAFE (SHORT)

SMOOTHED

$0.4-0.5$

LOW-WAISTED

21.5

16.8

SMOOTHED \& BURNISHED; PLAIN

CIRCULAR/SLIGHTLY CONCAVE

8.0

BURNISHED

Associated Funerary Object; Huddleston Burial 12, 5/11/1940; Social Hill - Deceiper phase 
Artifact No. / Old Cat. No. / Site No.

Vessel Form

ARAS/HSU Digital Photo

Type

Decoration

Rim :: Body

Paste

Temper

Core Color (Munsell)

Exterior Color (Munsell)

Interior Color (Munsell)

Vessel Overall Height (cm)

Vessel Overall Weight (g)

Vessel Maximum Diameter (cm)

Volume (liters)

Usewear/Sooting/Condition

Shape/Description

Lip Treatment/Shape/Angle/Thickness Orifice Exterior Diameter (cm)

Rim/Neck Height (cm)

Neck Mid/Base Exterior Diameter (cm)

Rim/Neck Shape/Angle

Rim/Neck Surface/Decorative Treatment

Rim/Neck/Body Thickness (cm)

Body Shape

Body Maximum Diameter (cm)

Body Height $(\mathrm{cm})$

Body Surface/Decorative Treatment

Base Shape/Curvature

Base Diameter/Height ( $\mathrm{cm})$

Base Surface/Decorative Treatment

Appendages/Handles

Notes
2-40/2211 / 3HS19

jar, tall rim

3504, N21721

Military Road Incised

incised/punctated

Beloit 9 :: Bard 1

HARD, SILTY, COMPACT

GROG, MED SIZE \& ABUNDANT; FEW SURFACE VOIDS

LIGHT YELLOWISH BROWN (10YR6/4), VERY DARK GRAY (10YR3/1) (CORE MODE E)

VERY DARK GRAY (10YR3/1), YELLOWISH RED (5YR4/6-5/6)

VERY DARK GRAY (10YR3/1)

19.0

1106

17.6

BLACKENED INT, SOME SOOTING EXT, FIRECLOUDING; VESSEL RECONSTRUCTED WITH GLUE AND PLASTER IN PAST, SOME PCS REGLUED; 2211 INKED NEAR BASE

TALL RIM JAR, LARGE, INCISED, FLARED RIM AND FLAT BOTTOM

FLAT, SLIGHTLY EVERTED

17.6

6.5

CONCAVE, VERTICAL

SMOOTHED EXT, BURNISHED INT, INCISED \& PUNCTATED, HORIZONTAL TRAILED LINES WITH VERTICAL PUNCTATIONS

$0.5-0.7$

SUBGLOBULAR

17.4

12.5

BURNISHED INT/SMOOTHED EXT; INCISED \& PUNCTATED, INC LINES HORIZONTAL AND MEANDERING, SEP BY ROWS OF VERTICAL PUNCTATES

CIRCULAR/FLAT

9.6

BURNISHED

Associated Funerary Object; Huddleston Burial 8, 4/20/1940; early Mid-Ouachita phase 
JEC Hodges Collection, 77-1

Artifact No. / Old Cat. No. / Site No.

Vessel Form

ARAS/HSU Digital Photo

Type

Decoration

$\operatorname{Rim}::$ Body

Paste

Temper

Core Color (Munsell)

Exterior Color (Munsell)

Interior Color (Munsell)

Vessel Overall Height $(\mathrm{cm})$

Vessel Overall Weight (g)

Vessel Maximum Diameter (cm)

Volume (liters)

Usewear/Sooting/Condition

Shape/Description

Lip Treatment/Shape/Angle/Thickness

Orifice Exterior Diameter $(\mathrm{cm})$

Rim/Neck Height (cm)

Neck Mid/Base Exterior Diameter (cm)

Rim/Neck Shape/Angle

Rim/Neck Surface/Decorative Treatment

Rim/Neck/Body Thickness (cm)

Body Shape

Body Maximum Diameter $(\mathrm{cm})$

Body Height $(\mathrm{cm})$

Body Surface/Decorative Treatment

Base Shape/Curvature

Base Diameter/Height $(\mathrm{cm})$

Base Surface/Decorative Treatment

Appendages/Handles

Notes
2-41 / 2198 / 3HS19

jar, short rim

3529

Emory Punctated-Incised?

incised/punctated

Caldwell 9 (sim.) :: Belmont 8 (type)

HARD, FINE, SILTY, COMPACT

SHELL (SOME LEACHED)

VERY DARK GRAY (10YR3/1), LIGHT YELLOWISH BROWN (10YR6/4)

BLACK (10YR2/1), VERY DARK GRAY (10YR3/1), DARK GRAY (10YR4/1)

VERY DARK GRAY (10YR3/1), YELLOWISH BROWN (10YR5/4)

9.5

296

12.5

BASE VERY ERODED (CHIPPED? FLATTENED); VESSEL RECONSTRUCTED WITH GLUE IN PAST, MANY FRAGMENTS MISSING, PROBE HOLE IN BASE

GLOBULAR, SHORT-RIM JAR WITH FLATTENED/ERODED BASE AND FLARED LIP

FLARED, ROUNDED

11.1

1.5

SMOOTHED \& PUNCTATED, 3 ROWS OF PUNCTATIONS

0.5

LOW-WAISTED (SLIGHTLY SQUAT GLOBULAR)

12.5

8.0

SMOOTHED; INCISED, CONCENTRIC HALF-CIRCLES EXTENDING DOWN FROM RIM, PATTERN REPEATS $3 \mathrm{X}$

OVAL/FLATTENED (ERODED)

$3.9 \times 4.5$

Huddleston Burial 5, 4/9/1940; Social Hill phase 
Artifact No. / Old Cat. No. / Site No.

Vessel Form

ARAS/HSU Digital Photo

Type

Decoration

$\operatorname{Rim}::$ Body

Paste

Temper

Core Color (Munsell)

Exterior Color (Munsell)

Interior Color (Munsell)

Vessel Overall Height $(\mathrm{cm})$

Vessel Overall Weight (g)

Vessel Maximum Diameter (cm)

Volume (liters)

Usewear/Sooting/Condition

Shape/Description

Lip Treatment/Shape/Angle/Thickness

Orifice Exterior Diameter $(\mathrm{cm})$

Rim/Neck Height (cm)

Neck Mid/Base Exterior Diameter $(\mathrm{cm})$

Rim/Neck Shape/Angle

Rim/Neck Surface/Decorative Treatment

Rim/Neck/Body Thickness (cm)

Body Shape

Body Maximum Diameter (cm)

Body Height (cm)

Body Surface/Decorative Treatment

Base Shape/Curvature

Base Diameter/Height $(\mathrm{cm})$

Base Surface/Decorative Treatment

Appendages/Handles

Notes
2-42/2232/3HS19

jar, tall rim

$3527, \mathrm{~N} 22550$

incised/brushed

Alaska 1 :: Danbury 2

HARD, SILTY, COMPACT

SHELL, COARSE \& ABUNDANT

PALE BROWN (10YR6/3) (CORE MODE G)

V DK GRAY BRN (10YR3/2), V DK GRAY (10YR3/1), DK GRAY BRN (10YR4/2)

BLACK (10YR2/1), DARK GRAY (10YR4/1), GRAY (10YR5/1)

10.4

240

10.5

FIRECLOUDING; VESSEL RECONSTRUCTED WITH GLUE IN PAST, SEVERAL FRAGMENTS RIM MISSING/UNATTACHED IN BAG; 2232 INKED NEAR BASE

TALL-RIM JAR BRUSHED WITH FLARED LIP AND FLATTENED BASE

FLAT, EVERTED, 0.2

10.5

4.0

CONCAVE, OUTSLANTED

SMOOTHED, INCISED \& BRUSHED, HORIZONTAL BRUSHING BETW 2 ROWS OF DIAGONAL INCISED LINES

$0.3-0.4$

SUBGLOBULAR

9.7

6.4

SMOOTHED; BRUSHED, MANY HORIZONTAL BRUSHED LINES AROUND BODY, LOWER 1.5CM PLAIN

CIRCULAR/FLAT

5.2

Associated Funerary Object; Huddleston Burial 10, 5/5/1940; Social Hill phase 
Artifact No. / Old Cat. No. / Site No.

Vessel Form

ARAS/HSU Digital Photo

Type

Decoration

Rim :: Body

Paste

Temper

Core Color (Munsell)

Exterior Color (Munsell)

Interior Color (Munsell)

Vessel Overall Height $(\mathrm{cm})$

Vessel Overall Weight (g)

Vessel Maximum Diameter (cm)

Volume (liters)

Usewear/Sooting/Condition

Shape/Description

Lip Treatment/Shape/Angle/Thickness

Orifice Exterior Diameter (cm)

Rim/Neck Height (cm)

Neck Mid/Base Exterior Diameter $(\mathrm{cm})$

Rim/Neck Shape/Angle

Rim/Neck Surface/Decorative Treatment

Rim/Neck/Body Thickness (cm)

Body Shape

Body Maximum Diameter (cm)

Body Height $(\mathrm{cm})$

Body Surface/Decorative Treatment

Base Shape/Curvature

Base Diameter/Height (cm)

Base Surface/Decorative Treatment

Appendages/Handles

Notes
$2-43 / 2209 / 3$ HS 19

jar, tall rim

$3525, \mathrm{~N} 21466$

Military Road Incised

incised/punctated

Bethel 1 :: Bard 27 (sim.)

HARD, SILTY, COMPACT, MICA IN PASTE

GROG, COARSE \& MEDIUM ABUNDANT

DARK GRAY (10YR4/1), YELLOWISH RED (5YR5/6) (CORE MODE A)

YELLOWISH RED (5YR5/6), VERY DARK GRAY (10YR3/1), GRAY (10YR5/1)

YELLOWISH RED (5YR5/6), VERY DARK GRAY (10YR3/1), GRAY (10YR5/1)

30.0

2734

24.8

LIGHT SOOTING INT/EXT RIM, FIRECLOUDING; VESSEL RECONSTRUCTED WITH GLUE IN PAST, SEVERAL FRAGMENTS MISSING, SMALL PROBE? HOLE IN RIM; 2209 INKED NEAR BASE

LARGE, TALL-RIM JAR WITH FLAT BASE.

FLAT, NONE

24.8

8.0

STRAIGHT, OUTSLANTED

SMOOTHED, INCISED (TRAILED) \& PUNCTATE (TOOL), 4 MEANDERING LINES BETW 2-3 ROWS OF VERTICAL PUNCTATES ABOVE AND BELOW

$0.7-1.0$

ELONGATED

24.1

22.0

SMOOTHED; INCISED (TRAILED) \& PUNCTATED (TOOL), COMBINATION OF STRAIGHT, MEANDERING, DIAGONAL TRAILED LINES, VERTICAL PUNCTATES

CIRCULAR/FLAT

11.1

Associated Funerary Object; Huddleston Burial 8, 4/20/1940; early Mid-Ouachita phase 
Artifact No. / Old Cat. No. / Site No.

Vessel Form

ARAS/HSU Digital Photo

Type

Decoration

Rim :: Body

Paste

Temper

Core Color (Munsell)

Exterior Color (Munsell)

Interior Color (Munsell)

Vessel Overall Height $(\mathrm{cm})$

Vessel Overall Weight (g)

Vessel Maximum Diameter $(\mathrm{cm})$

Volume (liters)

Usewear/Sooting/Condition

Shape/Description

Lip Treatment/Shape/Angle/Thickness Orifice Exterior Diameter $(\mathrm{cm})$

Rim/Neck Height (cm)

Neck Mid/Base Exterior Diameter (cm)

Rim/Neck Shape/Angle

Rim/Neck Surface/Decorative Treatment

Rim/Neck/Body Thickness (cm)

Body Shape

Body Maximum Diameter (cm)

Body Height $(\mathrm{cm})$

Body Surface/Decorative Treatment

Base Shape/Curvature

Base Diameter/Height (cm)

Base Surface/Decorative Treatment

Appendages/Handles

Notes
2-44 / 2204 / 3HS19

jar, tall rim

3582, N21751

Foster/Caney?

incised/punctated

Alfred $6::$ Caney 5

HARD, SILTY, COMPACT

SHELL (PARTLY LEACHED), COARSE \& ABUNDANT

BLACK (10YR2/1) (CORE MODE B)

LT BROWNISH GRAY (10YR6/2), DK GRAY (10YR4/1), PALE BROWN (10YR6/3)

LIGHT REDDISH BROWN (5YR6/4), VERY DARK GRAY (10YR3/1), DARK GRAY (10YR4/1)

24.5

1404

23.8

RESIDUE EXT RIM, SOME SOOT EXT BODY, BLACKENED INT, FIRECLOUDS; PARTIAL VESSEL RECONSTRUCTED WITH GLUE IN PAST, FEW CHIPS FROM LIP, PCS MISSING FROM BASE; 2204 INKED ON SIDE

\section{TALL RIM JAR, FLARED RIM AND FLATTENED BASE}

FLAT, EVERTED

23.8

11.0

VERTICAL, OUTSLANTED

SMOOTHED, INCISED, 9 ROWS OF DIAGONALLY INCISED LINES, SPACED

0.4

SUBGLOBULAR

20.3

13.5

SMOOTHED; PUNCTATED, VERTICAL ROWS OF PAIRED PUNCTATES

CIRCULAR/FLAT

8.7

Associated Funerary Object; Huddleston Burial 7, 4/13/1940; Social Hill phase 
JEC Hodges Collection, 77-1

Artifact No. / Old Cat. No. / Site No.

Vessel Form

ARAS/HSU Digital Photo

Type

Decoration

Rim :: Body

Paste

Temper

Core Color (Munsell)

Exterior Color (Munsell)

Interior Color (Munsell)

Vessel Overall Height $(\mathrm{cm})$

Vessel Overall Weight (g)

Vessel Maximum Diameter $(\mathrm{cm})$

Volume (liters)

Usewear/Sooting/Condition

Shape/Description

Lip Treatment/Shape/Angle/Thickness

Orifice Exterior Diameter (cm)

Rim/Neck Height (cm)

Neck Mid/Base Exterior Diameter (cm)

Rim/Neck Shape/Angle

Rim/Neck Surface/Decorative Treatment

Rim/Neck/Body Thickness (cm)

Body Shape

Body Maximum Diameter $(\mathrm{cm})$

Body Height $(\mathrm{cm})$

Body Surface/Decorative Treatment

Base Shape/Curvature

Base Diameter/Height $(\mathrm{cm})$

Base Surface/Decorative Treatment

Appendages/Handles

Notes
$2-45 / 2200 / 3 H S 19$

jar, tall rim

3589

Foster Trailed-Incised variant?

incised/appliqued

Brevard 2 (sim.) :: Bellarmine 7 (type)

HARD, SILTY, COMPACT

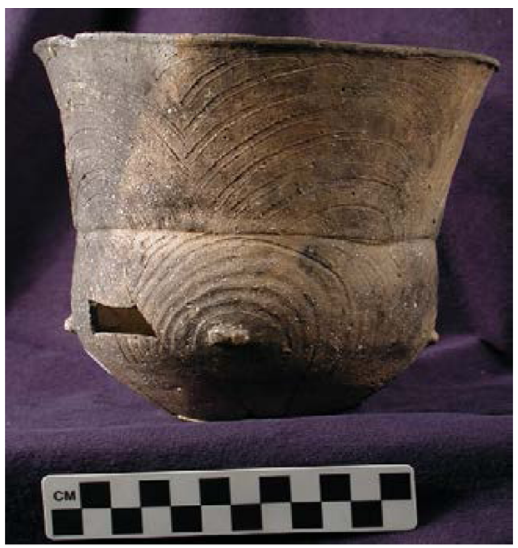

SHELL (SOME LEACHED), COARSE \& ABUNDANT

DARK GRAY (10YR4/1)

BLACK (10YR2/1), VERY DARK GRAY (10YR3/1), GRAY (10YR6/1)

VERY DARK GRAY (10YR3/1), REDDISH YELLOW (7.5YR6/6)

15.0

436

16.1

FIRECLOUDING; VESSEL IS RECONSTRUCTED WITH GLUE IN PAST, MANY FRAGMENTS MISSING

TALL-RIMMED JAR, SQUAT-BODIED WITH FLAT BASE

FLARED

16.1

7.0

CONCAVE, OUTSLANTED

SMOOTHED, INCISED/TRAILED, BROAD ARCS AROUND RIM

0.4

SUBGLOBULAR

13.1

8.0

INCISED/TRAILED, CONCENTRIC ARCS ON UPPER BODY, 2-PRONG APPLIQUE NODES REPEATS $4 \mathrm{X}$

CIRCULAR/FLAT

5.3

4 2-PRONG APPLIQUE NODES AROUND BODY

Huddleston Burial 5, 4/9/1940; Social Hill phase 


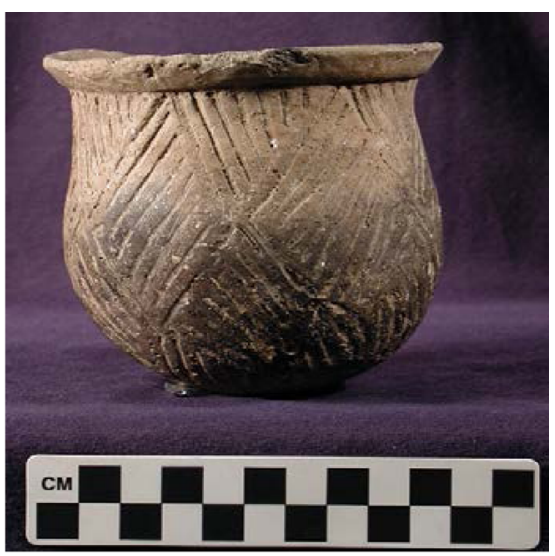

Artifact No. / Old Cat. No. / Site No.

Vessel Form

ARAS/HSU Digital Photo

Type

Decoration

Rim :: Body

Paste

Temper

Core Color (Munsell)

Exterior Color (Munsell)

Interior Color (Munsell)

Vessel Overall Height $(\mathrm{cm})$

Vessel Overall Weight $(\mathrm{g})$

Vessel Maximum Diameter (cm)

Volume (liters)

Usewear/Sooting/Condition

Shape/Description

Lip Treatment/Shape/Angle/Thickness Orifice Exterior Diameter (cm)

Rim/Neck Height (cm)

Neck Mid/Base Exterior Diameter (cm)

Rim/Neck Shape/Angle

Rim/Neck Surface/Decorative Treatment

Rim/Neck/Body Thickness (cm)

Body Shape

Body Maximum Diameter (cm)

Body Height $(\mathrm{cm})$

Body Surface/Decorative Treatment

Base Shape/Curvature

Base Diameter/Height (cm)

Base Surface/Decorative Treatment

Appendages/Handles

Notes
$2-46 / 2250 / 3$ HS 19

jar, short rim

3612

DeRoche Incised var. Central

incised

plain :: Antioch 14 (type)

HARD, SILTY, COMPACT

SHELL (SOME LEACHED), COARSE \& ABUNDANT

DARK GRAY (10YR4/1)

BLACK (10YR2/1), VERY DARK GRAY (10YR3/1), DARK GRAY (10YR4/1)

BROWN (10YR5/3), BLACK (10YR2/1), DARK GRAY (10YR4/1)

10.3

241

10.2

FIRECLOUDING; VESSEL INTACT EXCEPT MISSING ABOUT 1/3 OF THE RIM

SHORT-RIM JAR, INCISED, FLARED RIM AND ROUNDED BASE

FLATTENED, EVERTED (FLARED)

10.2

1.0

STRAIGHT, OUTSLANTED

SMOOTHED, PLAIN

0.4

SUBGLOBULAR

9.6

9.3

INCISED, DIAONAL LINE-FILLED DIAMOND AND TRIANGLE PATTERNS

UNDISTINGUISHED/CONVEX

Huddleston Burial 13, 5/19/1940; Deceiper phase 
JEC Hodges Collection, 77-1

Artifact No. / Old Cat. No. / Site No.

Vessel Form

ARAS/HSU Digital Photo

Type

Decoration

Rim :: Body

Paste

Temper

Core Color (Munsell)

Exterior Color (Munsell)

Interior Color (Munsell)

Vessel Overall Height $(\mathrm{cm})$

Vessel Overall Weight $(\mathrm{g})$

Vessel Maximum Diameter $(\mathrm{cm})$

Volume (liters)

Usewear/Sooting/Condition

Shape/Description

Lip Treatment/Shape/Angle/Thickness

Orifice Exterior Diameter $(\mathrm{cm})$

Rim/Neck Height (cm)

Neck Mid/Base Exterior Diameter (cm)

Rim/Neck Shape/Angle

Rim/Neck Surface/Decorative Treatment

Rim/Neck/Body Thickness (cm)

Body Shape

Body Maximum Diameter (cm)

Body Height $(\mathrm{cm})$

Body Surface/Decorative Treatment

Base Shape/Curvature

Base Diameter/Height $(\mathrm{cm})$

Base Surface/Decorative Treatment

Appendages/Handles

Notes
$2-47$ / 2197 / 3HS19

bowl, simple

3615

plain

SOFT, SILTY, COMPACT

SHELL (SOME LEACHED), MED TO COARSE, ABUNDANT

VERY DARK GRAY (10YR3/1)

BLACK (10YR2/1), V DK GRAYISH BROWN (10YR3/2), PALE BROWN (10YR6/3)

BLACK (10YR2/1), DARK GRAY (10YR4/1), LIGHT YELLOWISH BROWN (10YR6/4)

7.4

213

16.5

FIRECLOUDING; VESSEL RECONSTRUCTED WITH GLUE IN PAST, MANY SMALL FRAGMENTS MISSING

PLAIN BOWL WITH FLARED RIM AND FLATTENED BASE

FLATTENED, EVERTED (FLARED)

16.5

0.9

STRAIGHT, OUTSLANTED

SMOOTHED; PLAIN

0.4

CONVEX

15.7

6.5

SMOOTHED; PLAIN

CIRCULAR/ FLAT

3.3

Huddleston Burial 5, 4/9/1940; Social Hill phase 
JEC Hodges Collection, 77-1

Artifact No. / Old Cat. No. / Site No.

Vessel Form

ARAS/HSU Digital Photo

Type

Decoration

Rim :: Body

Paste

Temper

Core Color (Munsell)

Exterior Color (Munsell)

Interior Color (Munsell)

Vessel Overall Height $(\mathrm{cm})$

Vessel Overall Weight (g)

Vessel Maximum Diameter (cm)

Volume (liters)

Usewear/Sooting/Condition

Shape/Description

Lip Treatment/Shape/Angle/Thickness

Orifice Exterior Diameter $(\mathrm{cm})$

Rim/Neck Height (cm)

Neck Mid/Base Exterior Diameter (cm)

Rim/Neck Shape/Angle

Rim/Neck Surface/Decorative Treatment

Rim/Neck/Body Thickness (cm)

Body Shape

Body Maximum Diameter (cm)

Body Height (cm)

Body Surface/Decorative Treatment

Base Shape/Curvature

Base Diameter/Height (cm)

Base Surface/Decorative Treatment

Appendages/Handles

Notes
2-48 / 2199 / 3HS19

jar, tall rim

3617

Foster Trailed-Incised variant?

brushed/incised/appliqued

Defiance 1 (type) :: Belmont 6 (type)

HARD, SILTY, COMPACT

SHELL (SOME LEACHED), COARSE \& ABUNDANT

DARK GRAY (10YR4/1)

BLACK (10YR2/1), VERY DARK GRAY (10YR3/1), DARK GRAY (10YR4/1)

BLACK (10YR2/1), VERY DARK GRAY (10YR3/1), GRAY (10YR5/1)

22.3

1393

23.1

FIRECLOUDING; VESSEL RECONSTRUCTED WITH GLUE IN PAST, A FEW SHERDS MISSING INCL RIM SECTION

TALL-RIMMED LARGE JAR WITH U-SHAPED BODY, FLARED LIP, AND VERY SLIGHTLY FLATTENED BASE

FLATTENED, EVERTED (FLARED)

23.1

11.3

CONCAVE, OUTSLANTED

SMOOTHED; BRUSHED \& INCISED, CONNECTED ARCS AROUND RIM, ROW OF DIAGONAL SLASH INCISING AT BASE OF RIM

$0.7-0.5$

HIGH-WAISTED

20.6

11.0

SMOOTHED; INCISED/TRAILED, CONCENTRIC CIRCLES/SEMICIRCLES SURROUNDING 42 PRONG APPLIQUE NODES

CIRCULAR/FLAT (SLIGHTLY)

$\sim 7.0$

4 2-PRONG APPLIQUE NODES ON BODY

Huddleston Burial 5, 4/9/1940; Social Hill phase 
Artifact No. / Old Cat. No. / Site No.

Vessel Form

ARAS/HSU Digital Photo

Type

Decoration

Rim :: Body

Paste

Temper

Core Color (Munsell)

Exterior Color (Munsell)

Interior Color (Munsell)

Vessel Overall Height (cm)

Vessel Overall Weight (g)

Vessel Maximum Diameter $(\mathrm{cm})$

Volume (liters)

Usewear/Sooting/Condition

Shape/Description

Lip Treatment/Shape/Angle/Thickness

Orifice Exterior Diameter $(\mathrm{cm})$

Rim/Neck Height (cm)

Neck Mid/Base Exterior Diameter $(\mathrm{cm})$

Rim/Neck Shape/Angle

Rim/Neck Surface/Decorative Treatment

Rim/Neck/Body Thickness (cm)

Body Shape

Body Maximum Diameter (cm)

Body Height $(\mathrm{cm})$

Body Surface/Decorative Treatment

Base Shape/Curvature

Base Diameter/Height $(\mathrm{cm})$

Base Surface/Decorative Treatment

Appendages/Handles

Notes
2-49 / 2230 / 3HS19

bowl, carinated

3619, N22916

Sandford Punctated

punctated

Caldwell 3 :: plain

HARD, SILTY, COMPACT

SHELL (SOME LEACHED), FINE \& ABUNDANT + GROG, FINE \& SPARSE

VERY DARK GRAY (10YR3/1) (CORE MODE B)

GRAY (10YR5/1), PALE BROWN (10YR6/3), BLACK (10YR2/1)

BLACK (10YR2/1), VERY DARK GRAY (10YR3/1), DARK GRAY (10YR4/1)

10.3

834

23.7

BASE ROUGHENED ON EXT; FIRECLOUDING; VESSEL RECONSTRUCTED WITH GLUE IN PAST, SEVERAL FRAGMENTS MISSING OR IN BAG, PROBE HOLE IN BASE; 2230 INKED ON BASE

LARGE CARINATED BOWL, FLARED LIP AND ROUNDED BASE

BURNISHED, ROUNDED, EVERTED, 0.2

21.0

4.3

CONVEX, CARINATED

SMOOTHED \& BURNISHED, TOOL PUNCTATES, 5 ROWS OF VERTICAL OVAL PUNCTATES

$0.6-0.5$

CONVEX

23.7

6.0

SMOOTHED \& BURNISHED; PLAIN

UNDISTINGUISHED/CONVEX

Associated Funerary Object; Huddleston Burial 10, 5/5/1940; Social Hill phase 
JEC Hodges Collection, 77-1

Artifact No. / Old Cat. No. / Site No.

Vessel Form

ARAS/HSU Digital Photo

Type

Decoration

Rim :: Body

Paste

Temper

Core Color (Munsell)

Exterior Color (Munsell)

Interior Color (Munsell)

Vessel Overall Height (cm)

Vessel Overall Weight ( $\mathrm{g}$ )

Vessel Maximum Diameter $(\mathrm{cm})$

Volume (liters)

Usewear/Sooting/Condition

Shape/Description

Lip Treatment/Shape/Angle/Thickness Orifice Exterior Diameter (cm)

Rim/Neck Height (cm)

Neck Mid/Base Exterior Diameter (cm)

Rim/Neck Shape/Angle

Rim/Neck Surface/Decorative Treatment

Rim/Neck/Body Thickness (cm)

Body Shape

Body Maximum Diameter (cm)

Body Height $(\mathrm{cm})$

Body Surface/Decorative Treatment

Base Shape/Curvature

Base Diameter/Height (cm)

Base Surface/Decorative Treatment

Appendages/Handles

Notes
2-50 / 2184 / 3HS19

jar, short rim

3621

Mound Tract Inc./Brush. (Cowhide Stamp.)

dentate stamped/incised/brushed

Ferris $1::$ Drury 1

HARD, SILTY, COMPACT

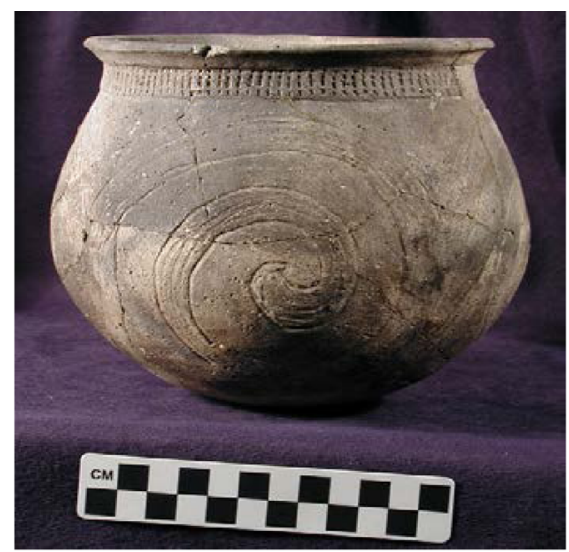

SHELL (SOME LEACHED), COARSE \& ABUNDANT

DARK GRAY (10YR4/1)

VERY DARK GRAY (10YR3/1), DARK GRAY (10YR4/1), PALE BROWN (10YR6/3)

VERY DARK GRAY (10YR3/1), LIGHT BROWNISH GRAY (10YR6/2), PALE BROWN

(10YR6/3)

14.7

430

16.2

FIRECLOUDING; VESSEL RECONSTRUCTED WITH GLUE IN PAST, SEVERAL SMALL PIECES MISSING FROM BODY

SMALL, SHORT RIM JAR WITH FLARED RIM AND ROUNDED BASE

ROUNDED, EVERTED (FLARED)

14.4

2.0

STRAIGHT, OUTSLANTED / STRAIGHT, INSLANTED

SMOOTHED; DENTATE STAMPING, VERTICAL STAMPING WITH 4-PRONG TOOL; HORIZ INCISED LINE BELOW

$0.5-0.3$

GLOBULAR

16.2

12.7

SMOOTHED; BRUSHED, MULTI-LINED SWIRL PATTERN, ZONED WITH TRAILED LINES, REPEATED $4 \mathrm{X}$

UNDISTINGUISHED/CONVEX

Huddleston Burial 3, 3/24/1940; Deceiper phase 
JEC Hodges Collection, 77-1

Artifact No. / Old Cat. No. / Site No.

Vessel Form

ARAS/HSU Digital Photo

Type

Decoration

Rim :: Body

Paste

Temper

Core Color (Munsell)

Exterior Color (Munsell)

Interior Color (Munsell)

Vessel Overall Height $(\mathrm{cm})$

Vessel Overall Weight (g)

Vessel Maximum Diameter $(\mathrm{cm})$

Volume (liters)

Usewear/Sooting/Condition

Shape/Description

Lip Treatment/Shape/Angle/Thickness

Orifice Exterior Diameter $(\mathrm{cm})$

Rim/Neck Height (cm)

Neck Mid/Base Exterior Diameter (cm)

Rim/Neck Shape/Angle

Rim/Neck Surface/Decorative Treatment

Rim/Neck/Body Thickness (cm)

Body Shape

Body Maximum Diameter (cm)

Body Height $(\mathrm{cm})$

Body Surface/Decorative Treatment

Base Shape/Curvature

Base Diameter/Height (cm)

Base Surface/Decorative Treatment

Appendages/Handles

Notes
$2-51 / 2256 / 3$ HS19

jar, tall rim

3631

incised/brushed

Alaska 1 :: Antioch 1

HARD, SILTY, COMPACT

SHELL (SOME LEACHED), COARSE \& ABUNDAN'T

DARK GRAY (10YR4/1)

BLACK (10YR2/1) SOOT, VERY DARK GRAY (10YR3/1), LIGHT GRAY (10YR7/2)

VERY DARK GRAY (10YR3/1), DARK GRAY (10YR4/1), GRAYISH BROWN (10YR5/2)

17.1

686

17.4

SOOTING ON UPPER BODY EXT/INT, FIRECLOUDING; VESSEL RECONSTRUCTED WITH GLUE IN PAST, SEVERAL FRAGMENTS MISSING

\section{TALL-RIM GLOBULAR JAR WITH FLARED RIM AND FLATTENED BASE}

FLATTENED, EVERTED (FLARED)

17.4

5.1

CONCAVE, OUTSLANTED

SMOOTHED; INCISED \& BRUSHED, HORIZONTAL BRUSHING BETW 2 ROWS OF DIAGONAL SLASH INCISING (SIM. TO FOSTER T-I)

$0.5-0.4$

SUB-GLOBULAR

16.4

12.0

SMOOTHED; INCISED, PATTERN OF DIAGONAL INCISED LINES FORMING NESTED TRIANGLES, PATTERN COVERS UPPER 3/4 BODY

CIRCULAR/FLAT

7.9

Huddleston Burial 15, 6/4/1940; Mid-Ouachita phase 
Artifact No. / Old Cat. No. / Site No.

Vessel Form

ARAS/HSU Digital Photo

Type

Decoration

Rim :: Body

Paste

Temper

Core Color (Munsell)

Exterior Color (Munsell)

Interior Color (Munsell)

Vessel Overall Height $(\mathrm{cm})$

Vessel Overall Weight (g)

Vessel Maximum Diameter (cm)

Volume (liters)

Usewear/Sooting/Condition

Shape/Description

Lip Treatment/Shape/Angle/Thickness

Orifice Exterior Diameter (cm)

Rim/Neck Height (cm)

Neck Mid/Base Exterior Diameter (cm)

Rim/Neck Shape/Angle

Rim/Neck Surface/Decorative Treatment

Rim/Neck/Body Thickness (cm)

Body Shape

Body Maximum Diameter $(\mathrm{cm})$

Body Height $(\mathrm{cm})$

Body Surface/Decorative Treatment

Base Shape/Curvature

Base Diameter/Height (cm)

Base Surface/Decorative Treatment

Appendages/Handles

Notes
$2-52 / 2186 / 3$ HS 19

jar, tall rim

3629

Foster Trailed-Incised

punctated/incised/appliqued

Carbon 1 :: Baker 3

HARD, SILTY, COMPACT

SHELL, COARSE \& ABUNDANT

BLACK (10YR2/1) (CORE MODE B)

BLACK (10YR2/1), V DK GRAY (10YR3/1), V DK GRAYISH BROWN (10YR3/2)

BLACK (10YR2/1), VERY DARK GRAYISH BROWN (10YR3/2), DARK GRAY (10YR4/1)

22.5

1026

25.4

SPALLING ON BASE (PRE-DEP?), BLACKENED RESIDUE INT RIM \& UPPER BODY, EXT BODY SOOTING; VESSEL RECONSTRUCTED WITH GLUE IN PAST, SEVERAL FRAGMENTS MISSING/UNATTACHED; 2186 INKED ON BASE

LARGE TALL-RIM JAR WITH FLARED RIM AND FLATTENED BASE

FLAT, SLIGHTLY EVERTED

25.4

9.1

CONCAVE, OUTSLANTED

SMOOTHED, PUNCTATED, 8 HORIZONTAL SPACED BANDS OF WEDGE SHAPED TOOL PUNCTATES

0.3

SUB-GLOBULAR

21.2

13.4

SMOOTHED (PORTIONS OF LOWER BODY BURNISHED); INCISED/TRAILED, LINES 3MM WIDE, 6 MM APART, 3 APPLIQUE NODES SURROUNDED BY CONCENTRIC CIRCLES, DECORATION ENDS 2.5CM ABOVE BASE

CIRCULAR/FLAT

7.5

33-NODE CLUSTERS APPLIQUED ON BODY

Associated Funerary Object; Huddleston Burial 4,3/26/1940 (Huddleston noted jar contained 'small animal bones \& muscle shells'); Social Hill - Deceiper phase 
Artifact No. / Old Cat. No. / Site No.

Vessel Form

ARAS/HSU Digital Photo

Type

Decoration

Rim :: Body

Paste

Temper

Core Color (Munsell)

Exterior Color (Munsell)

Interior Color (Munsell)

Vessel Overall Height $(\mathrm{cm})$

Vessel Overall Weight (g)

Vessel Maximum Diameter (cm)

Volume (liters)

Usewear/Sooting/Condition

Shape/Description

Lip Treatment/Shape/Angle/Thickness

Orifice Exterior Diameter (cm)

Rim/Neck Height (cm)

Neck Mid/Base Exterior Diameter $(\mathrm{cm})$

Rim/Neck Shape/Angle

Rim/Neck Surface/Decorative Treatment

Rim/Neck/Body Thickness (cm)

Body Shape

Body Maximum Diameter $(\mathrm{cm})$

Body Height $(\mathrm{cm})$

Body Surface/Decorative Treatment

Base Shape/Curvature

Base Diameter/Height (cm)

Base Surface/Decorative Treatment

Appendages/Handles

Notes
$2-53 / 2206 / 3$ HS19

bowl, simple

3633

plain

HARD, SILTY, COMPACT

SHELL (SOME LEACHED), MEDIUM SIZED \& ABUNDANT

VERY DARK GRAY (10YR3/1) (CORE MODE B)

VERY DARK GRAY (10YR3/1), DARK GRAY (10YR4/1), GRAY (10YR5/1)

DARK GRAY (10YR4/1), GRAY (10YR5/1), BROWN (10YR5/3)

10.8

463

16.0

1.1

ROUGHENED ON BASE EXT, SPALLING ON BASE INTERIOR (POSTDEP?); MINOR GLUE REPAIR IN PAST, 1-2 SHERDS MISSING, RIM CHIPPED (FROM PROBE?); 2206 INKED NEAR BASE

SIMPLE BOWL WITH FLARED LIP AND SLIGHTLY FLATTENED BASE

ROUNDED, EVERTED

16.0

0.4

STRAIGHT, OUTSLANTED, CONTINUOUS WITH BODY

SMOOTHED; PLAIN

$0.3-0.5$

CONVEX

15.7

10.4

BURNISHED; PLAIN

UNDISTINGUISHED/CONVEX, SLIGHTLY FLATTENED

$\sim 6.0$

Associated Funerary Object; Huddleston Burial 7, 4/13/1940 (Huddleston noted bone awl and 'large muscle shell' found under 2206); Social Hill phase 
Artifact No. / Old Cat. No. / Site No.

Vessel Form

ARAS/HSU Digital Photo

Type

Decoration

Rim :: Body

Paste

Temper

Core Color (Munsell)

Exterior Color (Munsell)

Interior Color (Munsell)

Vessel Overall Height $(\mathrm{cm})$

Vessel Overall Weight (g)

Vessel Maximum Diameter (cm)

Volume (liters)

Usewear/Sooting/Condition

Shape/Description

Lip Treatment/Shape/Angle/Thickness

Orifice Exterior Diameter (cm)

Rim/Neck Height (cm)

Neck Mid/Base Exterior Diameter (cm)

Rim/Neck Shape/Angle

Rim/Neck Surface/Decorative Treatment

Rim/Neck/Body Thickness (cm)

Body Shape

Body Maximum Diameter (cm)

Body Height $(\mathrm{cm})$

Body Surface/Decorative Treatment

Base Shape/Curvature

Base Diameter/Height $(\mathrm{cm})$

Base Surface/Decorative Treatment

Appendages/Handles

Notes
$2-55$ / 393 / 3HS19

bowl, simple

3639

plain

HARD, SILTY, COMPACT

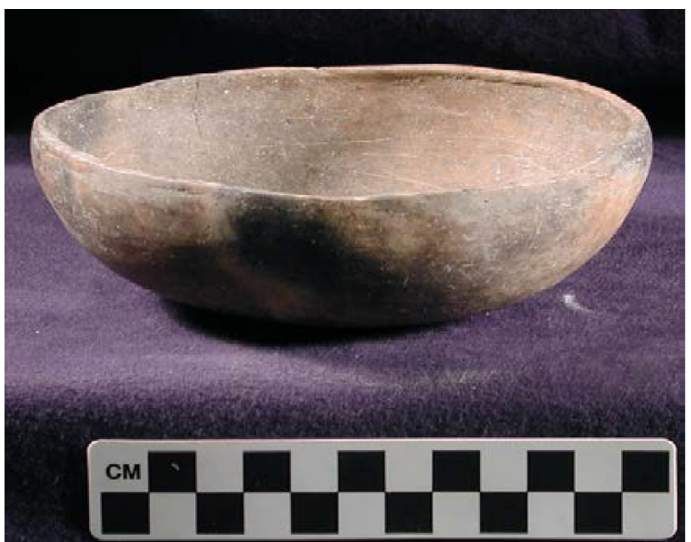

SHELL (SOME LEACHED), FINE/MED, ABUNDANT

DARK GRAY (10YR4/1)

REDDISH YELLOW (5YR6/6), LT REDDISH BROWN (5YR6/4), BLACK (10YR2/1)

REDDISH YELLOW (5YR6/6), LIGHT REDDISH BROWN (5YR6/4), VERY DARK GRAY (10YR3/1)

4.8

150

13.7

FIRECLOUDING; VESSEL INTACT EXCEPT FOR CRACK AND PROBE HOLE NEAR THE BASE

SIMPLE BOWL WITH FLATTENED RIM/LIP AND SLIGHTLY FLATTENED BASE

FLATTENED, NONE

13.5

0.4

CONVEX, VERTICAL; CONTINUOUS WITH BODY

SMOOTHED; PLAIN

0.3

CONVEX

13.7

4.45

SMOOTHED; PLAIN

UNDISTINGUISHED/CONVEX, SLIGHTLY FLATTENED

Hodges 393 (Hodges catalog: "small conical bowl, very shallow, plain, glazed, perfect, mound ... NE Arkadelphia Old River Bayou or Slough, Meador's Field"); Phillips photo 3810 (Hodges 393) 


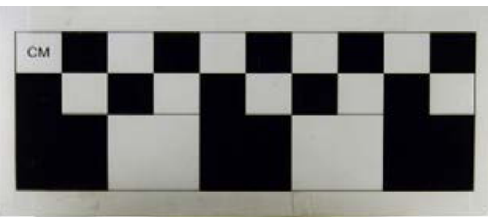

Artifact No. / Old Cat. No. / Site No.

Vessel Form

ARAS/HSU Digital Photo

Type

Decoration

Rim :: Body

Paste

Temper

Core Color (Munsell)

Exterior Color (Munsell)

Interior Color (Munsell)

Vessel Overall Height $(\mathrm{cm})$

Vessel Overall Weight (g)

Vessel Maximum Diameter $(\mathrm{cm})$

Volume (liters)

Usewear/Sooting/Condition

Shape/Description

Lip Treatment/Shape/Angle/Thickness

Orifice Exterior Diameter $(\mathrm{cm})$

Rim/Neck Height (cm)

Neck Mid/Base Exterior Diameter (cm)

Rim/Neck Shape/Angle

Rim/Neck Surface/Decorative Treatment

Rim/Neck/Body Thickness (cm)

Body Shape

Body Maximum Diameter $(\mathrm{cm})$

Body Height (cm)

Body Surface/Decorative Treatment

Base Shape/Curvature

Base Diameter/Height (cm)

Base Surface/Decorative Treatment

Appendages/Handles

Notes
2-56 / 2174 / 3HS19

jar, short rim

3654, N21559

Keno/Wallace?

incised

plain :: Buffalo 1 (sim.)

HARD, SILTY, COMPACT

SHELL (SOME LEACHED),FINE \& ABUNDANT

BLACK (10YR2/1) (CORE MODE B)

BLACK (10YR2/1), V DK GRAY (10YR3/1), DK GRAYISH BROWN (10YR4/2)

BLACK (10YR2/1), VERY DARK GRAY (10YR3/1), DARK GRAYISH BROWN (10YR4/2)

7.0

115

11.0

FIRECLOUDING; VESSEL RECONSTRUCTED WITH GLUE IN PAST \& REGLUED, SEVERAL FRAGMENTS MISSING/UNATTACHED; 2174 INKED ON BASE

SHORT-RIM JAR WITH FLARED RIM AND ROUNDED BASE

ROUNDED, SLIGHTLY EVERTED

11.0

1.9

CONCAVE, OUTSLANTED

SMOOTHED \& BURNISHED; PLAIN

0.3

CONVEX

9.7

5.1

SMOOTHED \& BURNISHED; INCISED, CLOSE SPACED, MEDIUM INCISED CONCENTRIC INTERLOCKING SCROLLS ( lines $1 \mathrm{~mm}$ wide, $3-5 \mathrm{~mm}$ apart), REPEATS 5X AROUND BODY; HORIZONTAL LINE AT RIM

Undistinguished/Convex

Associated Funerary Object; Huddleston Burial 1,3/23 or 3/24/1940 (Huddleston noted this vessel with child in grave); Deceiper phase 
Artifact No. / Old Cat. No. / Site No.

Vessel Form

ARAS/HSU Digital Photo

Type

Decoration

Rim :: Body

Paste

Temper

Core Color (Munsell)

Exterior Color (Munsell)

Interior Color (Munsell)

Vessel Overall Height (cm)

Vessel Overall Weight (g)

Vessel Maximum Diameter (cm)

Volume (liters)

Usewear/Sooting/Condition

Shape/Description

Lip Treatment/Shape/Angle/Thickness

Orifice Exterior Diameter (cm)

Rim/Neck Height (cm)

Neck Mid/Base Exterior Diameter (cm)

Rim/Neck Shape/Angle

Rim/Neck Surface/Decorative Treatment

Rim/Neck/Body Thickness (cm)

Body Shape

Body Maximum Diameter (cm)

Body Height $(\mathrm{cm})$

Body Surface/Decorative Treatment

Base Shape/Curvature

Base Diameter/Height $(\mathrm{cm})$

Base Surface/Decorative Treatment

Appendages/Handles

Notes
2-57 / 2173 / 3HS19

jar, short rim

3641, N21529

plain

HARD, SILTY, COMPACT

SHELL (SOME LEACHED), COARSE \& ABUNDANT

BLACK (10YR2/1), VERY DARK GRAY (10YR3/1), GRAY (10YR5/1)

VERY DARK GRAYISH BROWN (10YR3/2), DARK GRAY (10YR4/1), DARK GRAYISH BROWN (10YR4/2)

7.4

186

9.7

0.3

SOOT BELOW RIM INT, FIRECLOUDING; VESSEL INTACT, TWO SMALL CRACKS; 2173 INKED ON BASE

SMALL, SHORT-RIM JAR OR BOWL WITH FLARED RIM AND ROUNDED BASE

ROUNDED, SLIGHTLY EVERTED

9.7

1.8

CONCAVE, OUTSLANTED

SMOOTHED TO BURNISHED

0.5

SUB-GLOBULAR

9.2

5.6

SMOOTHED \& BURNISHED; PLAIN

Undistinguished/Convex

Associated Funerary Object; Huddleston Burial 1,3/23 or 3/24/1940 (Huddleston noted this vessel with child in grave); Deceiper phase 
JEC Hodges Collection, 77-1

Artifact No. / Old Cat. No. / Site No.

Vessel Form

ARAS/HSU Digital Photo

Type

Decoration

Rim :: Body

Paste

Temper

Core Color (Munsell)

Exterior Color (Munsell)

Interior Color (Munsell)

Vessel Overall Height $(\mathrm{cm})$

Vessel Overall Weight (g)

Vessel Maximum Diameter $(\mathrm{cm})$

Volume (liters)

Usewear/Sooting/Condition

Shape/Description

Lip Treatment/Shape/Angle/Thickness

Orifice Exterior Diameter $(\mathrm{cm})$

Rim/Neck Height (cm)

Neck Mid/Base Exterior Diameter (cm)

Rim/Neck Shape/Angle

Rim/Neck Surface/Decorative Treatment

Rim/Neck/Body Thickness (cm)

Body Shape

Body Maximum Diameter $(\mathrm{cm})$

Body Height $(\mathrm{cm})$

Body Surface/Decorative Treatment

Base Shape/Curvature

Base Diameter/Height (cm)

Base Surface/Decorative Treatment

Appendages/Handles

Notes
2-58 / 2254 / 3HS19

jar, short rim

1300

DeRoche Incised?

incised/punctated

Caldwell 10 ?:: Antioch 17

HARD, COMPACT

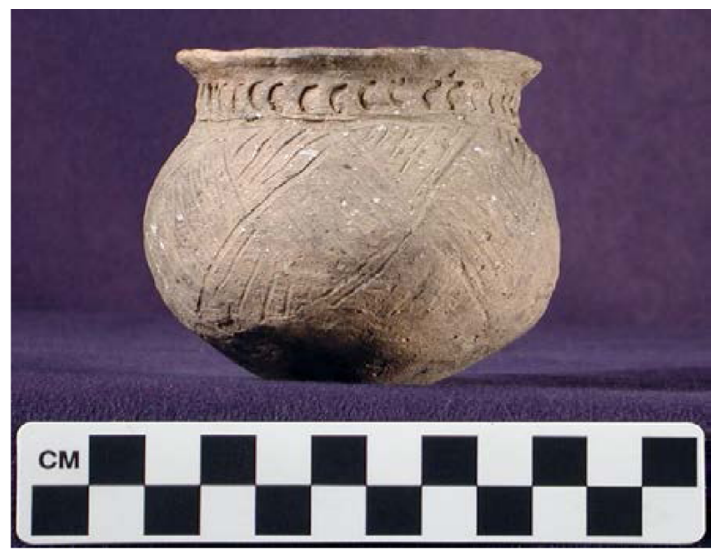

SHELL, MED TO COARSE \& ABUNDANT

LIGHT BROWN (7.5YR6/4), DARK GRAY (10YR4/1)

BROWN (7.5YR4/2)

7.0

112

7.8

FIRECLOUDING; INTACT VESSEL

MINI-JAR-SHORT RIM JAR, FLAT BASE, GLOBULAR BODY

ROUNDED, EVERTED (FLARED)

6.9

1.2

STRAIGHT, OUTSLANTED / STRAIGHT, VERTICAL

SMOOTHED, 1 ROW OF PUNCTATES AROUND NECK, CIRCULAR TOOL, 5MM APART

$0.5-0.4$

GLOBULAR

7.8

5.8

SMOOTHED; INCISED, INCISED LINES, ALTERNATING LINE-FILLED TRIANGLES AND DIAMONDS, HORIZONTAL LINE AT NECK; DESIGN COVERS 3/4 OF BODY

CIRCULAR/FLAT

2.0

Huddleston Burial 14, 6/1/1940; Deceiper phase 
JEC Hodges Collection, 77-1

Artifact No. / Old Cat. No. / Site No.

Vessel Form

ARAS/HSU Digital Photo

Type

Decoration

Rim :: Body

Paste

Temper

Core Color (Munsell)

Exterior Color (Munsell)

Interior Color (Munsell)

Vessel Overall Height $(\mathrm{cm})$

Vessel Overall Weight (g)

Vessel Maximum Diameter (cm)

Volume (liters)

Usewear/Sooting/Condition

Shape/Description

Lip Treatment/Shape/Angle/Thickness Orifice Exterior Diameter (cm)

Rim/Neck Height (cm)

Neck Mid/Base Exterior Diameter (cm)

Rim/Neck Shape/Angle

Rim/Neck Surface/Decorative Treatment

Rim/Neck/Body Thickness (cm)

Body Shape

Body Maximum Diameter $(\mathrm{cm})$

Body Height (cm)

Body Surface/Decorative Treatment

Base Shape/Curvature

Base Diameter/Height $(\mathrm{cm})$

Base Surface/Decorative Treatment

Appendages/Handles

Notes
2-59; 2234/3HS19

bottle

$3656, \mathrm{~N} 21499$

plain

HARD, SILTY, COMPACT

SHELL (SOME LEACHED), FINE \& ABUNDANT

VERY DARK GRAY (10YR3/1), DARK GRAY (10YR4/1) (CORE MODE C)

BLACK (10YR2/1), VERY DARK GRAY (10YR3/1), DARK GRAY (10YR4/1)

DARK GRAY (10YR4/1), LIGHT YELLOWISH BROWN (10YR6/4)

21.1

610

14.4

1.1

LIGHT ROUGHENING BASE EXT, SOME EROSION AROUND NECK, CHIPPED LIP; FIRECLOUDING; VESSEL INTACT; 2234 INKED NEAR BASE

BOTTLE; LONG NECK, GLOBULAR BODY, SL. FLARED RIM, AND FLATTENED BASE

ROUNDED, SLIGHTLY EVERTED

4.3

9.1

$4.3 / 4.7$

BULBOUS / SPOOL

SMOOTHED \& BURNISHED; PLAIN

0.5

GLOBULAR

14.4

12.0

SMOOTHED \& BURNISHED; PLAIN

CIRCULAR/FLAT

5.6

Associated Funerary Object; Huddleston Burial 10, 5/5/1940; Social Hill phase 
JEC Hodges Collection, 77-1

Artifact No. / Old Cat. No. / Site No.

Vessel Form

ARAS/HSU Digital Photo

Type

Decoration

Rim :: Body

Paste

Temper

Core Color (Munsell)

Exterior Color (Munsell)

Interior Color (Munsell)

Vessel Overall Height $(\mathrm{cm})$

Vessel Overall Weight (g)

Vessel Maximum Diameter (cm)

Volume (liters)

Usewear/Sooting/Condition

Shape/Description

Lip Treatment/Shape/Angle/Thickness

Orifice Exterior Diameter $(\mathrm{cm})$

Rim/Neck Height (cm)

Neck Mid/Base Exterior Diameter (cm)

Rim/Neck Shape/Angle

Rim/Neck Surface/Decorative Treatment

Rim/Neck/Body Thickness (cm)

Body Shape

Body Maximum Diameter $(\mathrm{cm})$

Body Height $(\mathrm{cm})$

Body Surface/Decorative Treatment

Base Shape/Curvature

Base Diameter/Height (cm)

Base Surface/Decorative Treatment

Appendages/Handles

Notes
2-60 / B186 / 3HS19

bowl, carinated

Friendship Engraved var. Freeman

engraved

Elaine 3 :: plain

HARD, SILTY, COMPACT, MICA IN PASTE

GROG, FINE \& ABUNDANT + SHELL (LEACHED), FINE \& SPARSE

RED (2.5YR5/8), V DK GRAY (10YR3/1), DK GRAY (10YR4/1), CORE MODE C

BLACK (10YR2/1), DARK GRAY (10YR4/1), BROWNISH YELLOW (10YR6/6)

BLACK (10YR2/1), DARK GRAY (10YR4/1), GRAYISH BROWN (10YR5/2)

$\sim 10.0$

571

$\sim 22.5$

FIRECLOUDING; CAT. AS SHERD LOT, PARTIAL VESSEL RECONSTRUCTED WITH GLUE

CARINATED BOWL WITH FLARED RIM

ROUNDED, EVERTED, 0.3

$\sim 22.0$

5.2

STRAIGHT, INSLANTED/CARINATED

SMOOTHED, ENGRAVED, 3 HORIZONTAL PANALS OF OVALS WITH TICKED LINES CROSSHATCHING BACKGROUND AND BETWEEN

$0.5-0.6$

CONVEX

$\sim 22.5$

$\sim 4.8$

SMOOTHED \& BURNISHED; PLAIN

UNDISTINGUISHED/CONVEX

Hodges Bag 186 [indicates broken pottery] (Hodges catalog: "Meador farm, Roher Md?") 


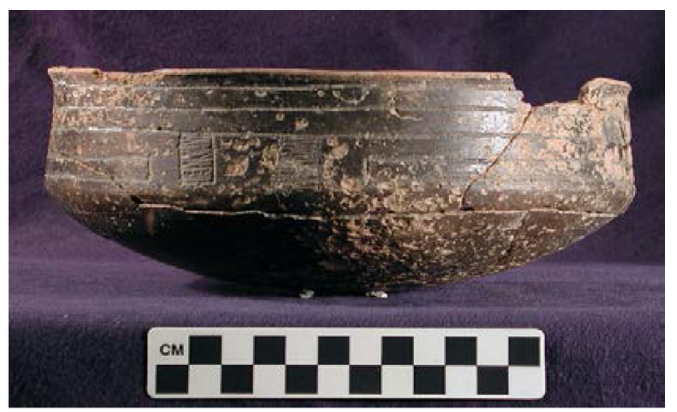

Artifact No. / Old Cat. No. / Site No.

Vessel Form

ARAS/HSU Digital Photo

Type

Decoration

Rim :: Body

Paste

Temper

Core Color (Munsell)

Exterior Color (Munsell)

Interior Color (Munsell)

Vessel Overall Height (cm)

Vessel Overall Weight (g)

Vessel Maximum Diameter (cm)

Volume (liters)

Usewear/Sooting/Condition

Shape/Description

Lip Treatment/Shape/Angle/Thickness

Orifice Exterior Diameter $(\mathrm{cm})$

Rim/Neck Height (cm)

Neck Mid/Base Exterior Diameter (cm)

Rim/Neck Shape/Angle

Rim/Neck Surface/Decorative Treatment

Rim/Neck/Body Thickness (cm)

Body Shape

Body Maximum Diameter (cm)

Body Height $(\mathrm{cm})$

Body Surface/Decorative Treatment

Base Shape/Curvature

Base Diameter/Height $(\mathrm{cm})$

Base Surface/Decorative Treatment

Appendages/Handles

Notes
2-61 / B186 / 3HS19

bowl, carinated

3660

Friendship Engraved variant?

engraved

Elaine? (ungramm.) :: plain

HARD, SILTY, COMPACT

SHELL (SOME LEACHED), FINE AND ABUNDANT

DARK GRAY (10YR4/1)

BLACK (10YR2/1), V DK GRAY (10YR3/1), V DK GRAYISH BROWN (10YR3/2)

VERY DARK GRAYISH BROWN (10YR3/2), DARK GRAY (10YR4/1), BLACK (10YR2/1)

9.2

451

20.7

CAT. AS SHERD LOT, RECONSTRUCTED WITH GLUE, A FEW SHERDS UNATTACHED; SPALLING LIKELY POST-DEP

CARINATED BOWL WITH FLARED RIM AND ROUNDED BASE

FLATTENED, EVERTED (FLARED)

20.5

4.6

STRAIGHT, CARINATED

SMOOTHED \& ENGRAVED, TWO HORIZONTAL PANELS OF LONG RECTANGLES AND CROSSHATCHED SQUARES

$0.4-0.6$

CONVEX

20.7

4.6

SMOOTHED \& BURNISHED; PLAIN

UNDISTINGUISHED/CONVEX

Hodges Bag 186 [indicates broken pottery] (Hodges catalog: "Meador farm, Roher Md?") 
JEC Hodges Collection, 77-1

Artifact No. / Old Cat. No. / Site No.

Vessel Form

ARAS/HSU Digital Photo

Type

Decoration

Rim :: Body

Paste

Temper

Core Color (Munsell)

Exterior Color (Munsell)

Interior Color (Munsell)

Vessel Overall Height $(\mathrm{cm})$

Vessel Overall Weight (g)

Vessel Maximum Diameter (cm)

Volume (liters)

Usewear/Sooting/Condition

Shape/Description

Lip Treatment/Shape/Angle/Thickness Orifice Exterior Diameter $(\mathrm{cm})$

Rim/Neck Height (cm)

Neck Mid/Base Exterior Diameter (cm)

Rim/Neck Shape/Angle

Rim/Neck Surface/Decorative Treatment

Rim/Neck/Body Thickness (cm)

Body Shape

Body Maximum Diameter $(\mathrm{cm})$

Body Height $(\mathrm{cm})$

Body Surface/Decorative Treatment

Base Shape/Curvature

Base Diameter/Height (cm)

Base Surface/Decorative Treatment

Appendages/Handles

Notes
2-67 / 2249 / 3HS19

bowl, carinated

3658

plain

SOFT, GRANULAR, CRUMBLY

SHELL (SOME LEACHED), COARSE AND ABUNDANT

VERY DARK GRAY (10YR3/1)

V DK GRAYISH BRN (10YR3/2), BROWN (10YR5/3), YELLOWISH BRN (10YR5/4)

DARK GRAY (10YR4/1), GRAYISH BROWN (10YR5/2), LIGHT YELLOWISH BROWN (10YR6/4)

8.3

433

18.7

ERODED; PARTIAL VESSEL, RECONSTRUCTED WITH GLUE, SEVERAL FRAGMENTS MISSING/UNATTACHED IN BAG

CARINATED BOWL WITH CURVED RIM AND ROUNDED BASE

ROUNDED, SLIGHTLY EVERTED (FLARED)

18.0

2.8

CONCAVE, VERTICAL/CARINATED

SMOOTHED; PLAIN

$0.6-0.5$

CONVEX

18.7

5.5

SMOOTHED; PLAIN

UNDISTINGUISHED/CONVEX

Huddleston Burial 13, 5/19/1940; Deceiper phase 
JEC Hodges Collection, 77-1

Artifact No. / Old Cat. No. / Site No.

Vessel Form

ARAS/HSU Digital Photo

Type

Decoration

Rim :: Body

Paste

Temper

Core Color (Munsell)

Exterior Color (Munsell)

Interior Color (Munsell)

Vessel Overall Height (cm)

Vessel Overall Weight (g)

Vessel Maximum Diameter (cm)

Volume (liters)

Usewear/Sooting/Condition

Shape/Description

Lip Treatment/Shape/Angle/Thickness

Orifice Exterior Diameter (cm)

Rim/Neck Height (cm)

Neck Mid/Base Exterior Diameter (cm)

Rim/Neck Shape/Angle

Rim/Neck Surface/Decorative Treatment

Rim/Neck/Body Thickness (cm)

Body Shape

Body Maximum Diameter (cm)

Body Height $(\mathrm{cm})$

Body Surface/Decorative Treatment

Base Shape/Curvature

Base Diameter/Height $(\mathrm{cm})$

Base Surface/Decorative Treatment

Appendages/Handles

Notes
2-70 / B197 / 3HS19

bottle (reused?)

3661

Keno Trailed

incised

missing :: Belhaven 1 or 14 ?

SOFT, GRANULAR, CRUMBLY

SHELL (SOME LEACHED), COARSE \& ABUNDANT

DARK GRAY (10YR4/1), BLACK (10YR2/1)

DARK GRAY (10YR4/1), GRAY (10YR5/1-6/1)

DARK GRAY (10YR4/1), DARK GRAYISH BROWN (10YR4/2)

16.5

409

NECK BROKEN/SMOOTHED FOR REUSE? SURFACE OF BODY WORN, SCRATCHED; PARTIAL VESSEL, RECONSTRUCTED WITH GLUE, SEVERAL SHERDS UNATTACHED/IN BAG

COMPOUND BOTTLE WITH BROKEN NECK, POSSIBLY A FLATTENED BASE

FLATTENED (BROKEN AND SMOOTHED?)

4.8 (neck)

3.1

UPPER NECK MISSING / LOWER NECK INSLANTING

SMOOTHED, VERTICAL LINES?

$0.5-0.8$

SUB-GLOBULAR

N/A

13.4

SMOOTHED; INCISED, INTERLOCKING SCROLL PATTERN REPEATED 4X, TRACE RED PIGMENT IN LINES

FLATTENED?

Hodges Bag 197 [indicates broken pottery] (Hodges catalog: "interesting variety, Meador farm ... Rohr Mound, mixed") 
Artifact No. / Old Cat. No. / Site No.

Vessel Form

ARAS/HSU Digital Photo

Type

Decoration

Rim :: Body

Paste

Temper

Core Color (Munsell)

Exterior Color (Munsell)

Interior Color (Munsell)

Vessel Overall Height $(\mathrm{cm})$

Vessel Overall Weight (g)

Vessel Maximum Diameter (cm)

Volume (liters)

Usewear/Sooting/Condition

Shape/Description

Lip Treatment/Shape/Angle/Thickness

Orifice Exterior Diameter $(\mathrm{cm})$

Rim/Neck Height (cm)

Neck Mid/Base Exterior Diameter $(\mathrm{cm})$

Rim/Neck Shape/Angle

Rim/Neck Surface/Decorative Treatment

Rim/Neck/Body Thickness (cm)

Body Shape

Body Maximum Diameter $(\mathrm{cm})$

Body Height $(\mathrm{cm})$

Body Surface/Decorative Treatment

Base Shape/Curvature

Base Diameter/Height $(\mathrm{cm})$

Base Surface/Decorative Treatment

Appendages/Handles

Notes
2-71/319/3HS19

bowl, carinated

3663

punctated/engraved

Elaine? (ungramm.) :: plain

HARD, SILTY, COMPACT

SHELL, FINE \& ABUNDANT

DARK GRAY (10YR4/1)

V DK GRAYISH BRN (10YR3/2), V DK GRAY (10YR3/1), DK GRAY (10YR4/1)

GRAYISH BROWN (10YR5/2), LIGHT YELLOWISH BROWN (10YR6/4)

6.3

150

12.5

PARTIAL VESSEL, RECONSTRUCTED WITH GLUE

SMALL, CARINATED BOWL, ROUNDED BASE

FLATTENED, NONE

12.5

3.0

VERTICAL, CARINATED

SMOOTHED; PUNCTATED \& ENGRAVED, PUNCTATE-FILLED OVALS X 4 (ONLY 2 REMAIN), SEP. BY CROSSHATCHING

$0.4-0.5$

CONVEX

12.4

3.3

SMOOTHED \& BURNISHED; PLAIN

UNDISTINGUISHED/CONVEX
Hodges 319 (Hodges catalog: "cazuela bowl, unusual design, almost perfect, mound on Old River Bayou NE of Ark.") 


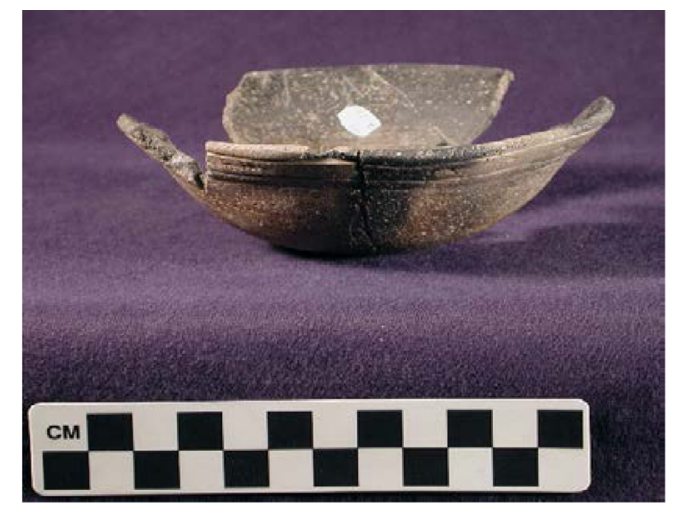

Artifact No. / Old Cat. No. / Site No.

Vessel Form

ARAS/HSU Digital Photo

Type

Decoration

$\operatorname{Rim}::$ Body

Paste

Temper

Core Color (Munsell)

Exterior Color (Munsell)

Interior Color (Munsell)

Vessel Overall Height (cm)

Vessel Overall Weight (g)

Vessel Maximum Diameter (cm)

Volume (liters)

Usewear/Sooting/Condition

Shape/Description

Lip Treatment/Shape/Angle/Thickness

Orifice Exterior Diameter (cm)

Rim/Neck Height (cm)

Neck Mid/Base Exterior Diameter (cm)

Rim/Neck Shape/Angle

Rim/Neck Surface/Decorative Treatment

Rim/Neck/Body Thickness (cm)

Body Shape

Body Maximum Diameter $(\mathrm{cm})$

Body Height $(\mathrm{cm})$

Body Surface/Decorative Treatment

Base Shape/Curvature

Base Diameter/Height (cm)

Base Surface/Decorative Treatment

Appendages/Handles

Notes
2-72 / B197 / 3HS19

bowl, simple

3665

engraved/punctated

Cornell 3, Case 4 :: plain

HARD, SILTY, COMPACT

SHELL (SOME LEACHED), FINE \& ABUNDANT

GRAYISH BROWN (10YR5/2)

BLACK (10YR2/1), V DK GRAYISH BROWN (10YR3/2), DK GRAY (10YR4/1)

BLACK (10YR2/1), GRAYISH BROWN (10YR5/2), 'BROWN (10YR4/3)

4.2

71

12.2

FIRECLOUDING; PARTIAL VESSEL, RECONSTRUCTED WITH GLUE

SMALL OPEN BOWL, 'HELMET' SHAPE

FLATTENED, NONE; NOTCHING ON TOP OF LIP

12.2

1.3

STRAIGHT, OUTSLANTED

SMOOTHED \& BURNISHED; 2 ENGRAVED LINES BELOW LIP WITH LINE OF PUNCTATES BETWEEN

0.4

CONVEX

10.1

2.9

SMOOTHED \& BURNISHED; PLAIN

UNDISTINGUISHED/CONVEX

Hodges Bag 197 [indicates broken pottery] (Hodges catalog: "interesting variety, Meador farm ... Rohr Mound, mixed") 


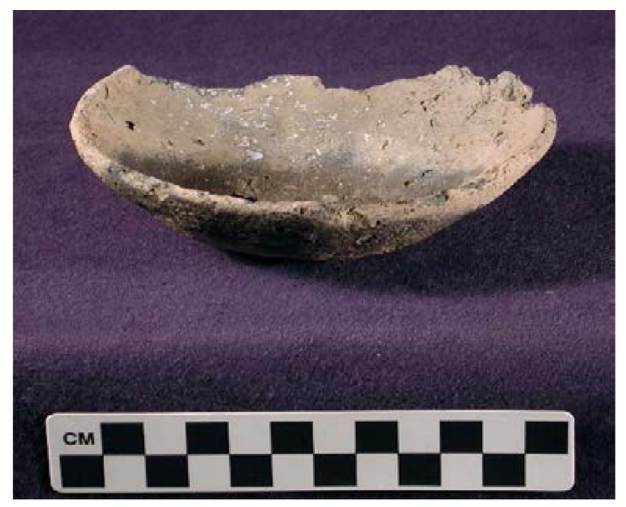

Artifact No. / Old Cat. No. / Site No.

Vessel Form

ARAS/HSU Digital Photo

Type

Decoration

Rim :: Body

Paste

Temper

Core Color (Munsell)

Exterior Color (Munsell)

Interior Color (Munsell)

Vessel Overall Height $(\mathrm{cm})$

Vessel Overall Weight (g)

Vessel Maximum Diameter (cm)

Volume (liters)

Usewear/Sooting/Condition

Shape/Description

Lip Treatment/Shape/Angle/Thickness

Orifice Exterior Diameter $(\mathrm{cm})$

Rim/Neck Height (cm)

Neck Mid/Base Exterior Diameter $(\mathrm{cm})$

Rim/Neck Shape/Angle

Rim/Neck Surface/Decorative Treatment

Rim/Neck/Body Thickness (cm)

Body Shape

Body Maximum Diameter $(\mathrm{cm})$

Body Height $(\mathrm{cm})$

Body Surface/Decorative Treatment

Base Shape/Curvature

Base Diameter/Height $(\mathrm{cm})$

Base Surface/Decorative Treatment

Appendages/Handles

Notes
2-74 / B197 / 3HS19

bowl, simple

3667

plain

SOFT, GRANULAR, COMPACT

SHELL (SOME LEACHED), COARSE \& ABUNDANT

BLACK (10YR2/1)

BLACK (10YR2/1), VERY DARK GRAY (10YR3/1), DARK GRAY (10YR4/1)

BLACK (10YR2/1), VERY DARK GRAYISH BROWN (10YR3/2), GRAYISH BROWN (10YR5/2)

4.0

95

12.1

FIRECLOUDING; CHIPPING AROUND RIM, PROBE HOLE IN BOTTOM

SMALL, SHALLOW BOWL WITH ROUNDED LIP AND ROUNDED BASE

ROUNDED, NONE

12.1

STRAIGHT, OUTSLANTED

SMOOTHED; PLAIN

0.5

CONVEX

12.1

4.0

SMOOTHED; PLAIN

UNDISTINGUISHED/CONVEX

Hodges Bag 197 [indicates broken pottery] (Hodges catalog: "interesting variety, Meador farm ... Rohr Mound, mixed") 
Artifact No. / Old Cat. No. / Site No.

Vessel Form

ARAS/HSU Digital Photo

Type

Decoration

$\operatorname{Rim}::$ Body

Paste

Temper

Core Color (Munsell)

Exterior Color (Munsell)

Interior Color (Munsell)

Vessel Overall Height $(\mathrm{cm})$

Vessel Overall Weight (g)

Vessel Maximum Diameter (cm)

Volume (liters)

Usewear/Sooting/Condition

Shape/Description

Lip Treatment/Shape/Angle/Thickness

Orifice Exterior Diameter (cm)

Rim/Neck Height (cm)

Neck Mid/Base Exterior Diameter (cm)

Rim/Neck Shape/Angle

Rim/Neck Surface/Decorative Treatment

Rim/Neck/Body Thickness (cm)

Body Shape

Body Maximum Diameter $(\mathrm{cm})$

Body Height (cm)

Body Surface/Decorative Treatment

Base Shape/Curvature

Base Diameter/Height $(\mathrm{cm})$

Base Surface/Decorative Treatment

Appendages/Handles

Notes
3-3 / 2858 / 3CL83

beaker

1829,3694

notched lip

Cornell $10::$ plain

SOFT, SMOOTH, CRUMBLY

SHELL (SOME LEACHED), COARSE \& ABUNDANT

YELLOWISH BROWN (10YR5/6), DARK YELLOWISH BROWN (10YR4/4)

BROWNISH YELLOW (10YR6/6), BLACK (10YR2/1), YELLOW BRN (10YR5/8)

GRAY (10YR5/1)

7.9

139

11.2

FIRECLOUDING; ERODED AREA ON EXT.; PARTIAL VESSEL RECONSTR. WITH GLUE, MISSING ALMOST ENTIRE BASE; 2858 INKED LOWER BODY

DEEP, CYLINDRICAL BOWL OR BEAKER WITH SLIGHTLY CONVEX SIDES; CONTINUOUS RIM/BODY

FLAT, NONE; NARROW PUNCTATES PLACED DIAGONALLY ON TOP OF LIP

11.2

STRAIGHT, OUTSLANTED (SLIGHTLY)

SMOOTHED, PLAIN

$0.4-0.5$

ELONGATED

11.2

7.9

SMOOTHED; PLAIN

CIRCULAR, FLAT

Huddleston Grave 9; 11/21/1943; Mid-Ouachita phase 
JEC Hodges Collection, 77-1

Artifact No. / Old Cat. No. / Site No.

Vessel Form

ARAS/HSU Digital Photo

Type

Decoration

Rim :: Body

Paste

Temper

Core Color (Munsell)

Exterior Color (Munsell)

Interior Color (Munsell)

Vessel Overall Height $(\mathrm{cm})$

Vessel Overall Weight (g)

Vessel Maximum Diameter (cm)

Volume (liters)

Usewear/Sooting/Condition

Shape/Description

Lip Treatment/Shape/Angle/Thickness

Orifice Exterior Diameter $(\mathrm{cm})$

Rim/Neck Height (cm)

Neck Mid/Base Exterior Diameter (cm)

Rim/Neck Shape/Angle

Rim/Neck Surface/Decorative Treatment

Rim/Neck/Body Thickness (cm)

Body Shape

Body Maximum Diameter $(\mathrm{cm})$

Body Height $(\mathrm{cm})$

Body Surface/Decorative Treatment

Base Shape/Curvature

Base Diameter/Height (cm)

Base Surface/Decorative Treatment

Appendages/Handles

Notes
3-4 / 2844 / 3CL83

bottle (broken, reused as seed jar)

3698

Belcher Engraved var. Soda Lake

engraved

plain :: Elizabeth 1

HARD, SILTY, COMPACT

GROG \& BONE \& SHELL (LEACHED), FINE \& SPARSE

RED (2.5YR5/8), REDDISH BLACK (2.5YR2.5/1), YELLOWISH BROWN (10YR5/4)

10.7

628

16.5

NECK BROKEN AND SMOOTHED FOR REUSE, BODY SLIGHTLY WORN; FIRECLOUDING

SEED JAR (ORIGINALLY BOTTLE), FLATTENED LIP (SMOOTHED BREAK), SQUAT GLOBULAR BODY, FLAT BASE

BROKEN/FLATTENED/SMOOTHED

3.4

$0.7-0.5$

LOW-WAISTED

16.5

10.2

SMOOTHED, BURNISHED; ENGRAVED, CONCENTRIC CIRCLES X4, ALTERNATING DASHED AND SOLID LINES, SPACE FILLING CROSSHATCHING \& BALL BELOW NECK

CIRCULAR/FLAT

4.5

Huddleston Grave 1, 11/20/1943; Social Hill-Deceiper phase 
Artifact No. / Old Cat. No. / Site No.

Vessel Form

ARAS/HSU Digital Photo

Type

Decoration

Rim :: Body

Paste

Temper

Core Color (Munsell)

Exterior Color (Munsell)

Interior Color (Munsell)

Vessel Overall Height $(\mathrm{cm})$

Vessel Overall Weight ( $\mathrm{g}$ )

Vessel Maximum Diameter (cm)

Volume (liters)

Usewear/Sooting/Condition

Shape/Description

Lip Treatment/Shape/Angle/Thickness

Orifice Exterior Diameter (cm)

Rim/Neck Height $(\mathrm{cm})$

Neck Mid/Base Exterior Diameter (cm)

Rim/Neck Shape/Angle

Rim/Neck Surface/Decorative Treatment

Rim/Neck/Body Thickness (cm)

Body Shape

Body Maximum Diameter (cm)

Body Height $(\mathrm{cm})$

Body Surface/Decorative Treatment

Base Shape/Curvature

Base Diameter/Height (cm)

Base Surface/Decorative Treatment

Appendages/Handles

Notes
3-5/ $2861 / 3 C L 83$

bottle

3702, N21691

Hodges Engraved var. Hodges

engraved

plain :: Ebbet 6 (sim.)

HARD, SILTY, COMPACT

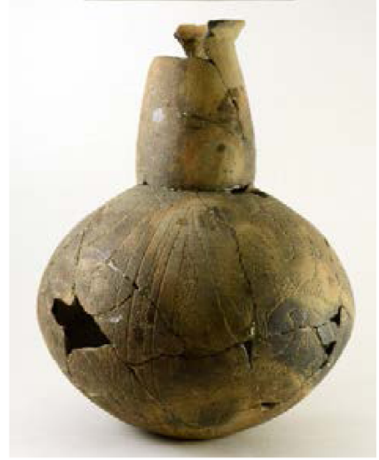

GROG, FINE \& ABUNDANT + SHELL (LEACHED), FINE \& MEDIUM ABUNDANT

VERY DARK GRAY (7.5YR3/1) (CORE MODE G)

V PALE BRN (10YR7/3), BROWN YELLOW (10YR6/5), DK GRAY BRN (10YR4/2)

VERY DARK GRAY (7.5YR3/1)

24.6

485

16.9

FIRECLOUDING; VESSEL RECONSTRUCTED WITH GLUE IN PAST (1944), SEVERAL SHERDS MISSING FROM BODY AND NECK; 2861 INKED ON BASE

BOTTLE; GLOBULAR SLIGHTLY SQUAT BOTTLE, SPOOL NECK, FLARED LIP, SLIGHTLY FLATTENED BASE

ROUNDED, EVERTED

5.2

10.0

$5.9 / 6.2$

bulbous / spool

burnished

0.4

GLOBULAR (SLIGHTLY SQUAT)

16.9

14.6

BURNISHED; ENGRAVED, INTERLOCKING HOOK ELEMENTS WITH U-SHAPE DESIGN TOP AND BOTTOM (TICKED LINE, CROSSHATCHING); TRACE RED PIGMENT IN ENGR LINES

\section{CIRCULAR/FLAT}

4.5

Associated Funerary Object; Huddleston Grave 10, 11/25/1943; Deceiper phase 
Artifact No. / Old Cat. No. / Site No.

Vessel Form

ARAS/HSU Digital Photo

Type

Decoration

Rim :: Body

Paste

Temper

Core Color (Munsell)

Exterior Color (Munsell)

Interior Color (Munsell)

Vessel Overall Height (cm)

Vessel Overall Weight (g)

Vessel Maximum Diameter (cm)

Volume (liters)

Usewear/Sooting/Condition

Shape/Description

Lip Treatment/Shape/Angle/Thickness

Orifice Exterior Diameter $(\mathrm{cm})$

Rim/Neck Height (cm)

Neck Mid/Base Exterior Diameter (cm)

Rim/Neck Shape/Angle

Rim/Neck Surface/Decorative Treatment

Rim/Neck/Body Thickness (cm)

Body Shape

Body Maximum Diameter (cm)

Body Height (cm)

Body Surface/Decorative Treatment

Base Shape/Curvature

Base Diameter/Height (cm)

Base Surface/Decorative Treatment

Appendages/Handles

Notes
3-6 / 2855 / 3CL83

bowl, carinated

3711, N22985

Sandford Punctated

punctated

Caldwell 3 or $6:$ : plain

HARD, SILTY, COMPACT

SHELL (LEACHED), FINE \& ABUNDANT + GROG, FINE \& MEDIUM ABUNDANT

GRAY (10YR5/1), PALE BROWN (10YR6/3) (CORE MODE B)

BLACK (10YR2/1), DARK GRAYISH BROWN (10YR4/2)

GRAYISH BROWN (10YR5/2), LIGHT BROWNISH GRAY (10YR6/2)

8.6

450

19.3

BASE EXT ROUGHENED; FIRECLOUDING ON BODY; PARTIAL VESSEL RECONSTRUCTED WITH GLUE, LARGE SHERD MISSING, GASH IN BASE; 2855 INKED ON BASE

CARINATED BOWL WITH PUNCTATED RIM, SLIGHTLY FLARED AND ROUNDED LIP, AND SLIGHTLY FLATTENED BASE

ROUNDED, EVERTED, 0.3

16.7

4.0

CONVEX, CARINATED

SMOOTHED; TOOL PUNCTATES, 5 ROWS OF VERTICAL TOOL PUNCTATES, HALF MOONSHAPED JABS RATHER THAN FINGERNAIL PUNCT

$0.5-0.7$

CONVEX

19.3

4.6

BURNISHED; PLAIN

UNDISTINGUISHED/FLATTENED

Associated Funerary Object; Huddleston Grave 6, 11/20-11/21/1943; Social Hill phase 


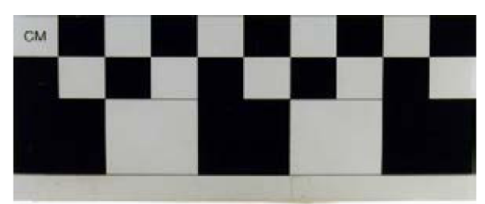

Artifact No. / Old Cat. No. / Site No.

Vessel Form

ARAS/HSU Digital Photo

Type

Decoration

$\operatorname{Rim}::$ Body

Paste

Temper

Core Color (Munsell)

Exterior Color (Munsell)

Interior Color (Munsell)

Vessel Overall Height $(\mathrm{cm})$

Vessel Overall Weight (g)

Vessel Maximum Diameter (cm)

Volume (liters)

Usewear/Sooting/Condition

Shape/Description

Lip Treatment/Shape/Angle/Thickness

Orifice Exterior Diameter (cm)

Rim/Neck Height (cm)

Neck Mid/Base Exterior Diameter (cm)

Rim/Neck Shape/Angle

Rim/Neck Surface/Decorative Treatment

Rim/Neck/Body Thickness (cm)

Body Shape

Body Maximum Diameter $(\mathrm{cm})$

Body Height (cm)

Body Surface/Decorative Treatment

Base Shape/Curvature

Base Diameter/Height (cm)

Base Surface/Decorative Treatment

Appendages/Handles

Notes
3-8 / 2853 / 3CL83

beaker or mini-jar

3731, N22682

punctated

plain :: Catawba 2?

HARD, SILTY, COMPACT

SHELL (LEACHED), COARSE \& SPARSE; OCCASIONAL HEMATITE PEBBLE

DARK GRAY (7.5YR4/1) (CORE MODE F)

REDDISH YELLOW (5YR6/6), GRAY (5YR5/1), PALE BROWN (10YR6/3)

REDDISH YELLOW (5YR6/6), PALE BROWN (10YR6/3)

5.3

85

6.7

0.1

VESSEL INTACT, CHIPPED LIP, HOLES FROM LEACHED TEMPER; 2853 INKED ON BASE

BEAKER OR MINI-JAR; VERY SMALL BEAKER WITH FLARED, SLIGHTLY FLATTENED LIP AND A FLAT BASE

FLAT, SLIGHTLY EVERTED, 0.4

6.7

1.0

STRAIGHT, OUTSLANTED

SMOOTHED

0.4

BARREL

6.6

4.3

SMOOTHED; PUNCTATES (TOOL PUNCTATES, HALF MOON SHAPED JABS) FORMING CONNECTING CONCENTRIC ARCS, PATTERN REPEATING 4X

CIRCULAR/FLAT

5.4

Associated Funerary Object; Huddleston Grave 6, 11/20-11/21/1943; Social Hill phase 
JEC Hodges Collection, 77-1

Artifact No. / Old Cat. No. / Site No.

Vessel Form

ARAS/HSU Digital Photo

Type

Decoration

Rim :: Body

Paste

Temper

Core Color (Munsell)

Exterior Color (Munsell)

Interior Color (Munsell)

Vessel Overall Height $(\mathrm{cm})$

Vessel Overall Weight (g)

Vessel Maximum Diameter $(\mathrm{cm})$

Volume (liters)

Usewear/Sooting/Condition

Shape/Description

Lip Treatment/Shape/Angle/Thickness

Orifice Exterior Diameter $(\mathrm{cm})$

Rim/Neck Height (cm)

Neck Mid/Base Exterior Diameter $(\mathrm{cm})$

Rim/Neck Shape/Angle

Rim/Neck Surface/Decorative Treatment

Rim/Neck/Body Thickness (cm)

Body Shape

Body Maximum Diameter $(\mathrm{cm})$

Body Height $(\mathrm{cm})$

Body Surface/Decorative Treatment

Base Shape/Curvature

Base Diameter/Height $(\mathrm{cm})$

Base Surface/Decorative Treatment

Appendages/Handles

Notes
3-9 / 2845 / 3CL83

effigy seed jar

3747, N22877

appliqued nodes

Harvey :: plain

MEDIUM-HARD, SILTY, COMPACT

SHELL (MOSTLY LEACHED), FINE \& SPARSE; OCCASIONAL PEBBLE IN PASTE

BROWN (10YR5/3) (CORE MODE B)

V DK GRAY BRN (10YR3/2), RED BROWN (5YR5/4), RED YELLOW (7.5YR6/6)

17.0

939

18.5

WEAR/ROUGHENING ON BASE, SURFACE EROSION RIM/BODY; FIRECLOUDING; VESSEL RECONSTRUCTED WITH GLUE IN PAST, LARGE CRACK IN SIDE, PROBE HOLE; 2845 INKED ON BASE

SEED JAR; EFFIGY SEED JAR WITH 4 APPLIQUE NODES ON RIM FOR FACIAL FEATURES, FLAT INCURVED LIP, GLOBULAR BODY, AND FLAT BASE

FLAT, NONE, 0.4

$4.1 \times 3.3$ CM OVAL

4.0

12.2

CONVEX, INSLANTED

BURNISHED, 4 APPLIQUE NODES ON RIM ( 2 VERTICAL IN FRONT AND 2 OPPOSING EACH OTHER ON EITHER SIDE) FORM FACE

0.6

LOW-WAISTED

18.5

13.0

BURNISHED; PLAIN

CIRCULAR/SLIGHTLY CONCAVE $(0.2 \mathrm{CM})$

7.0

4 APPLIQUE NODES ON RIM FORMING FACIAL EFFIGY

Associated Funerary Object; Huddleston Grave 3, 11/20/1943 (described by Huddleston as owl effigy); Social Hill phase; publ. Trubitt 2017 
Artifact No. / Old Cat. No. / Site No.

Vessel Form

ARAS/HSU Digital Photo

Type

Decoration

Rim :: Body

Paste

Temper

Core Color (Munsell)

Exterior Color (Munsell)

Interior Color (Munsell)

Vessel Overall Height (cm)

Vessel Overall Weight (g)

Vessel Maximum Diameter (cm)

Volume (liters)

Usewear/Sooting/Condition

Shape/Description

Lip Treatment/Shape/Angle/Thickness Orifice Exterior Diameter (cm)

Rim/Neck Height (cm)

Neck Mid/Base Exterior Diameter (cm)

Rim/Neck Shape/Angle

Rim/Neck Surface/Decorative Treatment

Rim/Neck/Body Thickness (cm)

Body Shape

Body Maximum Diameter $(\mathrm{cm})$

Body Height $(\mathrm{cm})$

Body Surface/Decorative Treatment

Base Shape/Curvature

Base Diameter/Height (cm)

Base Surface/Decorative Treatment

Appendages/Handles

Notes
3-10 / 2846 / 3CL83

jar, short rim

3753, N22754

incised

Barnard? or Bates 10 ? :: Baker 18

SOFT, SILTY, COMPACT

SHELL (LEACHED), MEDIUM TO COARSE \& ABUNDANT

VERY DARK GRAY (10YR3/1)

V DK GRAY (10YR3/1), DK GRAY BRN (10YR4/2), BROWN YELLOW (10YR6/6)

VERY DARK GRAY (10YR3/1), LIGHT YELLOWISH BROWN (10YR6/4)

12.5

409

14.3

1.1

ROUGHENING ON BODY INT. (USE?); SOOT/RESIDUE ON EXT. RIM BODY, INT. RIM BLACKENED; VESSEL INTACT EXCEPT FOR MINOR RECONSTRUCTION WITH GLUE IN PAST; 2846 INKED NEAR BASE

JAR; SHORT-RIM JAR WITH FLARED AND ROUNDED LIP, GLOBULAR BODY, AND ROUNDED BASE

ROUNDED, EVERTED, 0.3

12.9

2.3

\section{CONCAVE, OUTSLANTED}

SMOOTHED, INCISED, SINGLE INCISED HORIZ LINE AROUND BASE OF RIM/TOP OF BODY

$0.5-0.4$

GLOBULAR

14.3

10.2

SMOOTHED; INCISED, DRY PASTE, CONCENTRIC CIRCLES REPEAT 4X

UNDISTINGUISHED/CONVEX

Associated Funerary Object; Huddleston Grave 3, 11/20/1943; Social Hill phase 
Artifact No. / Old Cat. No. / Site No.

Vessel Form

ARAS/HSU Digital Photo

Type

Decoration

Rim :: Body

Paste

Temper

Core Color (Munsell)

Exterior Color (Munsell)

Interior Color (Munsell)

Vessel Overall Height $(\mathrm{cm})$

Vessel Overall Weight (g)

Vessel Maximum Diameter (cm)

Volume (liters)

Usewear/Sooting/Condition

Shape/Description

Lip Treatment/Shape/Angle/Thickness

Orifice Exterior Diameter $(\mathrm{cm})$

Rim/Neck Height (cm)

Neck Mid/Base Exterior Diameter (cm)

Rim/Neck Shape/Angle

Rim/Neck Surface/Decorative Treatment

Rim/Neck/Body Thickness (cm)

Body Shape

Body Maximum Diameter $(\mathrm{cm})$

Body Height $(\mathrm{cm})$

Body Surface/Decorative Treatment

Base Shape/Curvature

Base Diameter/Height $(\mathrm{cm})$

Base Surface/Decorative Treatment

Appendages/Handles

Notes
3-11 / 2865 / 3CL83

jar, tall rim

3755, N22613

Foster Trailed-Incised

incised/appliqued nodes

Andes $9::$ Baker 22

HARD, SILTY, COMPACT, MICA IN PASTE

SHELL (LEACHED), COARSE \& SPARSE, WITH OCCASIONAL HEMATITE PEBBLE

VERY DARK GRAY (7.5YR3/1) (CORE MODE B)

BLACK (10YR2/1), STRONG BROWN (7.5YR5/6), LT YELLOW BROWN (10YR6/4)

DARK GRAYISH BROWN (10YR4/2), BLACK (10YR2/1), VERY DARK GRAY (10YR3/1)

8.7

125

9.3

0.3

MINOR WEAR ON BASE; TRACE SOOT ON BODY EXT, FIRECLOUDING; ONE PC OF RIM MISSING, ANOTHER REATTACHED WITH GLUE IN PAST; 2865 INKED ON BASE

JAR; SMALL TALL-RIM JAR WITH WIDELY FLARED RIM, GLOBULAR BODY, AND ROUND BASE

FLAT, EVERTED, 0.3

9.3

3.1

CONCAVE, OUTSLANTED

SMOOTHED, INCISED, 3 HORIZONTAL BANDS WITH DIAGONAL LINES, HERRINGBONE DESIGN

$0.4-0.3$

GLOBULAR

8.2 AT NODES, 7.7 BODY

5.6

SMOOTHED, SOME BURNISHING EXT; INCISED, CONCENTRIC CIRCLES SURROUNDING CENTRAL NODE; PATTERN REPEATS 4X, DRY PASTE INCISING, ALMOST LOOKS LIKE ENGRAVING

UNDISTINGUISHED/CONVEX

1 SINGLE NODE IN CENTER OF EA CONCENTRIC PATTERN (4X)

Associated Funerary Object; Huddleston Grave 10, 11/25/1943; Deceiper phase 
Artifact No. / Old Cat. No. / Site No.

Vessel Form

ARAS/HSU Digital Photo

Type

Decoration

Rim :: Body

Paste

Temper

Core Color (Munsell)

Exterior Color (Munsell)

Interior Color (Munsell)

Vessel Overall Height (cm)

Vessel Overall Weight (g)

Vessel Maximum Diameter (cm)

Volume (liters)

Usewear/Sooting/Condition

Shape/Description

Lip Treatment/Shape/Angle/Thickness

Orifice Exterior Diameter (cm)

Rim/Neck Height (cm)

Neck Mid/Base Exterior Diameter $(\mathrm{cm})$

Rim/Neck Shape/Angle

Rim/Neck Surface/Decorative Treatment

Rim/Neck/Body Thickness (cm)

Body Shape

Body Maximum Diameter $(\mathrm{cm})$

Body Height $(\mathrm{cm})$

Body Surface/Decorative Treatment

Base Shape/Curvature

Base Diameter/Height $(\mathrm{cm})$

Base Surface/Decorative Treatment

Appendages/Handles

Notes
3-12 / 2866 / 3CL83

bowl, carinated

3759

Friendship Engraved var. Meador

engraved

Elwyn 3 (type) :: plain

HARD, SILTY, COMPACT

GROG \& GRIT, FINE \& SPARSE

REDDISH BROWN (5YR5/4)

GRAYISH BROWN (10YR5/2), BLACK (10YR2/1)

YELLOW (10YR7/6), REDDISH BROWN (5YR5/4), VERY DARK GRAY (10YR3/1)

8.3

654

18.7

BASE HEAVILY WORN, BLACKENED INT; VESSEL RECONSTRUCTED WITH GLUE, CRACKS, MINOR CHIPPING ON RIM

CARINATED BOWL WITH FLARED AND ROUNDED LIP, SHALLOW BODY AND ROUNDED BASE

ROUNDED, EVERTED (FLARED)

17.7

4.8

CONVEX, INSLANTED/CARINATED

BURNISHED; ENGRAVED, CROSSHATCHING SURROUNDING NEGATIVE DESIGN OF PANELS LINKED BY AN L-SHAPED ELEMENT

0.7

CONVEX

18.7

3.5

SMOOTHED \& BURNISHED; PLAIN

UNDISTINGUISHED/CONVEX

Huddleston Grave 15, 2/5/1944; Mid-Ouachita phase 
JEC Hodges Collection, 77-1

Artifact No. / Old Cat. No. / Site No.

Vessel Form

ARAS/HSU Digital Photo

Type

Decoration

Rim :: Body

Paste

Temper

Core Color (Munsell)

Exterior Color (Munsell)

Interior Color (Munsell)

Vessel Overall Height (cm)

Vessel Overall Weight (g)

Vessel Maximum Diameter $(\mathrm{cm})$

Volume (liters)

Usewear/Sooting/Condition

Shape/Description

Lip Treatment/Shape/Angle/Thickness

Orifice Exterior Diameter $(\mathrm{cm})$

Rim/Neck Height (cm)

Neck Mid/Base Exterior Diameter $(\mathrm{cm})$

Rim/Neck Shape/Angle

Rim/Neck Surface/Decorative Treatment

Rim/Neck/Body Thickness (cm)

Body Shape

Body Maximum Diameter (cm)

Body Height $(\mathrm{cm})$

Body Surface/Decorative Treatment

Base Shape/Curvature

Base Diameter/Height $(\mathrm{cm})$

Base Surface/Decorative Treatment

Appendages/Handles

Notes
3-13/2856 / 3CL83

bottle

3767

plain

MEDIUM, SILTY, COMPACT

SHELL (SOME LEACHED), FINE \& ABUNDANT

GRAY (7.5YR5/1)

DARK GRAY (10YR4/1), BLACK (10YR2/1), LIGHT BROWNISH GRAY (10YR6/2)

24.2

800

16.6

WORN/ERODED ON BODY (MODERATE) AND NECK (HEAVY); FIRECLOUDING; NECK REATTACHED TO BODY WITH GLUE

LONG-NECK BOTTLE WITH FLARED LIP, GLOBULAR BODY, AND FLAT BASE

ROUNDED, EVERTED (FLARED)

4.8

9.7

VERTICAL, SLIGHT SPOOL

SMOOTHED

0.5

GLOBULAR

16.6

14.5

SMOOTHED 7 BURNISHED; PLAIN

CIRCULAR/FLATTENED

5.0

Huddleston Grave 9; 11/21/1943; Mid-Ouachita phase 
JEC Hodges Collection, 77-1

Artifact No. / Old Cat. No. / Site No.

Vessel Form

ARAS/HSU Digital Photo

Type

Decoration

Rim :: Body

Paste

Temper

Core Color (Munsell)

Exterior Color (Munsell)

Interior Color (Munsell)

Vessel Overall Height $(\mathrm{cm})$

Vessel Overall Weight (g)

Vessel Maximum Diameter (cm)

Volume (liters)

Usewear/Sooting/Condition

Shape/Description

Lip Treatment/Shape/Angle/Thickness Orifice Exterior Diameter $(\mathrm{cm})$

Rim/Neck Height (cm)

Neck Mid/Base Exterior Diameter (cm)

Rim/Neck Shape/Angle

Rim/Neck Surface/Decorative Treatment

Rim/Neck/Body Thickness (cm)

Body Shape

Body Maximum Diameter $(\mathrm{cm})$

Body Height $(\mathrm{cm})$

Body Surface/Decorative Treatment

Base Shape/Curvature

Base Diameter/Height $(\mathrm{cm})$

Base Surface/Decorative Treatment

Appendages/Handles

Notes
3-14 / 2857 / 3CL83

jar, short rim

3771

Foster/Pease?

incised

Alfred 2 :: Alpha 1

HARD, SILTY, COMPACT

SHELL (SOME LEACHED), MEDIUM SIZED \& ABUNDANT

LIGHT BROWNISH GRAY (10YR6/2)

BLACK (10YR2/1), VERY DARK GRAYISH BROWN (10YR3/2), BROWN (10YR5/3)

LIGHT YELLOWISH BROWN (10YR6/4), BROWN (10YR5/3), DARK GRAYISH BROWN (10YR4/2)

10.2

182

10.1

LIGHT TO MODERATE WEAR/EROSION; FIRECLOUDING; VESSEL RECONSTRUCTED WITH GLUE, LARGE PC MISSING

SMALL SHORT-RIM JAR WITH FLARED LIP, NARROW BODY, AND FLAT BASE

FLATTENED, EVERTED (FLARED)

10.1

2.8

STRAIGHT, OUTSLANTED

INCISED, 2 BANDS OF DIAGONALLY INCISED LINES, WIDELY SEPARATED

$0.5-0.4$

ELONGATED

9.4

7.4

SMOOTHED; INCISED, HERRINGBONE PATTERN WITH VERTICAL LINES SEPARATING

CIRCULAR/FLAT

5.5

Huddleston Grave 9; 11/21/1943; Mid-Ouachita phase 


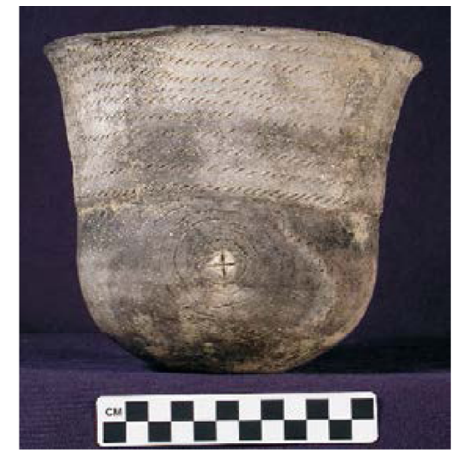

Artifact No. / Old Cat. No. / Site No.

Vessel Form

ARAS/HSU Digital Photo

Type

Decoration

Rim :: Body

Paste

Temper

Core Color (Munsell)

Exterior Color (Munsell)

Interior Color (Munsell)

Vessel Overall Height $(\mathrm{cm})$

Vessel Overall Weight (g)

Vessel Maximum Diameter $(\mathrm{cm})$

Volume (liters)

Usewear/Sooting/Condition

Shape/Description

Lip Treatment/Shape/Angle/Thickness

Orifice Exterior Diameter $(\mathrm{cm})$

Rim/Neck Height (cm)

Neck Mid/Base Exterior Diameter $(\mathrm{cm})$

Rim/Neck Shape/Angle

Rim/Neck Surface/Decorative Treatment

Rim/Neck/Body Thickness (cm)

Body Shape

Body Maximum Diameter (cm)

Body Height $(\mathrm{cm})$

Body Surface/Decorative Treatment

Base Shape/Curvature

Base Diameter/Height $(\mathrm{cm})$

Base Surface/Decorative Treatment

Appendages/Handles

Notes
3-15 / 2847 / 3CL83

jar, tall rim

1260

Foster Trailed-Incised

incised/punctated/applique

Concord 10 or Chattanooga 7 (sim.) :: Baker 23 (sim.)

MEDIUM-HARD, SMOOTH, COMPACT

SHELL (SOME LEACHED), MEDIUM SIZED \& ABUNDANT

DARK GRAY (7.5YR4/1) (CORE MODE B)

PINKISH GRAY (7.5YR6/2), DK BROWN (7.5YR3/2), V DK GRAY (7.5YR3/1)

BLACK (7.5YR2.5/1)

17.0

500

18.0

2.2

SOOTING RESIDUE ON VESSEL EXT, TRACE FROM BASE TO RIM, INTERIOR BLACKENED, FIRECLOUDING; VESSEL INTACT EXCEPT FOR LIP BREAK; 2847 INKED NEAR BASE

JAR; LARGE TALL RIM JAR-FLAT BASE-SUBGLOBULAR BODY-TALL OUTTURNED RIMPUNCTATE AND INCISED DESIGN, NODES

ROUNDED, EVERTED

18.0

8.0

CONCAVE, OUTSLANTED

SMOOTHED, PUNCTATED, 10 ROWS OF FINGERNAIL PUNCTATES, SPACED ROWS

$0.4-0.3$

SUB-GLOBULAR

14.3

9.0

SMOOTHED \& BURNISHED (NEAR BASE); INCISED LINE DECORATION- NARROW (1MM) LINES, WIDESPACED, CONCENTRIC CIRCLE DESIGN-3X REPEATED ON UPPER 3/4 OF BODY, 3 CENTRAL NODES (4 NODE CLUSTERS)

CIRCULAR/FLAT

6.0

3 CENTRAL NODES ON BODY (EACH ONE DIVIDED INTO 4 WITH CROSS)

Associated Funerary Object; Huddleston Grave 3, 11/20/1943; Social Hill phase 
Artifact No. / Old Cat. No. / Site No.

Vessel Form

ARAS/HSU Digital Photo

Type

Decoration

$\operatorname{Rim}::$ Body

Paste

Temper

Core Color (Munsell)

Exterior Color (Munsell)

Interior Color (Munsell)

Vessel Overall Height $(\mathrm{cm})$

Vessel Overall Weight (g)

Vessel Maximum Diameter (cm)

Volume (liters)

Usewear/Sooting/Condition

Shape/Description

Lip Treatment/Shape/Angle/Thickness

Orifice Exterior Diameter $(\mathrm{cm})$

Rim/Neck Height (cm)

Neck Mid/Base Exterior Diameter (cm)

Rim/Neck Shape/Angle

Rim/Neck Surface/Decorative Treatment

Rim/Neck/Body Thickness (cm)

Body Shape

Body Maximum Diameter $(\mathrm{cm})$

Body Height $(\mathrm{cm})$

Body Surface/Decorative Treatment

Base Shape/Curvature

Base Diameter/Height $(\mathrm{cm})$

Base Surface/Decorative Treatment

Appendages/Handles

Notes
3-16 / 2862 / 3CL83

bowl, carinated

3781, N22487

Ripley Engraved?

engraved

Erin (need new) :: plain

HARD, SILTY, COMPACT

SHELL (LEACHED), MEDIUM TO COARSE SIZE \& ABUNDANT + GROG, FINE TO MEDIUM SIZED \& SPARSE, OCCASIONAL PEBBLES

VERY DARK GRAY (7.5YR3/1) (CORE MODE G)

BLACK (10YR2/1), DARK BROWN (7.5YR3/2), REDDISH YELLOW (5YR6/6)

VERY DARK GRAY (10YR3/1)

10.0

348

17.0

LIGHT EROSION OVERALL; FIRECLOUDING ON EXT, BLACKENED INT.; PARTIAL VESSEL, RECONSTRUCTED WITH GLUE IN PAST, SEVERAL FRAGMENTS OF RIM AND BODY MISSING; 2862 INKED ON BODY EXT

BOWL; V-SHAPED CARINATED BOWL (TALL) WITH A FLARED AND SLIGHTLY

FLATTENED LIP AND A FLAT BASE

THICKENED, SLIGHTLY EVERTED, 0.4

17.0

4.5

\section{CONCAVE, CARINATED}

BURNISHED, ENGRAVED, REVERSE L-SHAPES, CROSSHATCHED INTERIORS, REPEATS 7X; TRACE RED PIGMENT IN ENGRAVING; SIM. VESSEL AT HELM

$0.5-0.4$

CONICAL

16.5

5.5

BURNISHED (SOMEWHAT ERODED); PLAIN

CIRCULAR/FLAT

6.5

Associated Funerary Object; Huddleston Grave 10, 11/25/1943; Deceiper phase 
JEC Hodges Collection, 77-1

Artifact No. / Old Cat. No. / Site No.

Vessel Form

ARAS/HSU Digital Photo

Type

Decoration

Rim :: Body

Paste

Temper

Core Color (Munsell)

Exterior Color (Munsell)

Interior Color (Munsell)

Vessel Overall Height $(\mathrm{cm})$

Vessel Overall Weight (g)

Vessel Maximum Diameter (cm)

Volume (liters)

Usewear/Sooting/Condition

Shape/Description

Lip Treatment/Shape/Angle/Thickness

Orifice Exterior Diameter $(\mathrm{cm})$

Rim/Neck Height (cm)

Neck Mid/Base Exterior Diameter (cm)

Rim/Neck Shape/Angle

Rim/Neck Surface/Decorative Treatment

Rim/Neck/Body Thickness (cm)

Body Shape

Body Maximum Diameter $(\mathrm{cm})$

Body Height (cm)

Body Surface/Decorative Treatment

Base Shape/Curvature

Base Diameter/Height $(\mathrm{cm})$

Base Surface/Decorative Treatment

Appendages/Handles

Notes
3-17 / 2848 / 3CL83

bowl, carinated

3783, N22457

Sandford Punctated

punctated

Caldwell 6 ?:: plain

HARD, MEDIUM, SILTY, COMPACT

SHELL (LEACHED), MEDIUM SIZED \& ABUNDANT

DARK GRAYISH BROWN (10YR4/2) (CORE MODE E)

RED (2.5YR5/6), BLACK (10YR2/1), PALE BROWN (10YR6/3)

VERY DARK GRAY (10YR3/1), BROWN (10YR4/3), PALE BROWN (10YR6/3)

7.9

435

20.6

SLIGHT WEAR/EROSION/LEACHING; FIRECLOUDING; VESSEL HAS MINOR RECONSTRUCTION WITH GLUE IN PAST, SEVERAL PIECES MISSING FROM RIM, CRACK; 2848 INKED ON BASE

CARINATED BOWL WITH INCURVED RIM, STRAIGHT ROUNDED LIP, AND SLIGHTLY FLATTENED BASE

ROUNDED, EVERTED, 0.3

18.9

2.9

CONVEX, CARINATED

SMOOTHED, TOOL PUNCTATES, 4 HORIZONTAL ROWS OF DEEP VERTICAL PUNCTATES, TOOL PUNCTATES, OVAL JABS

0.5

CONVEX

20.6

5.0

BURNISHED; PLAIN

UNDISTINGUISHED/FLAT

Associated Funerary Object; Huddleston Grave 3, 11/20/1943; Social Hill phase 
Artifact No. / Old Cat. No. / Site No.

Vessel Form

ARAS/HSU Digital Photo

Type

Decoration

$\operatorname{Rim}::$ Body

Paste

Temper

Core Color (Munsell)

Exterior Color (Munsell)

Interior Color (Munsell)

Vessel Overall Height (cm)

Vessel Overall Weight (g)

Vessel Maximum Diameter (cm)

Volume (liters)

Usewear/Sooting/Condition

Shape/Description

Lip Treatment/Shape/Angle/Thickness

Orifice Exterior Diameter $(\mathrm{cm})$

Rim/Neck Height (cm)

Neck Mid/Base Exterior Diameter (cm)

Rim/Neck Shape/Angle

Rim/Neck Surface/Decorative Treatment

Rim/Neck/Body Thickness (cm)

Body Shape

Body Maximum Diameter $(\mathrm{cm})$

Body Height $(\mathrm{cm})$

Body Surface/Decorative Treatment

Base Shape/Curvature

Base Diameter/Height $(\mathrm{cm})$

Base Surface/Decorative Treatment

Appendages/Handles

Notes
3-18 / 2843 / 3CL83

jar, short rim

3785

Cowhide/Foster?

dentate stamped/incised/applique

Ferris 1 :: Baker 2

HARD, SILTY, COMPACT

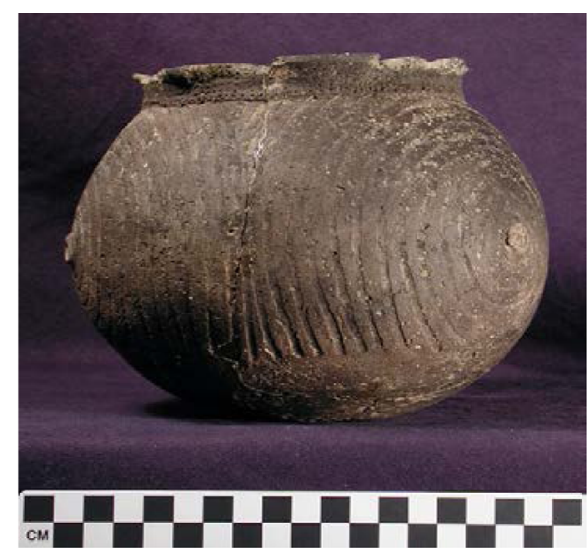

SHELL (SOME LEACHED), COARSE \& ABUNDANT

PALE BROWN (10YR6/3)

V DK GRAY (10YR3/1), PALE BROWN (10YR6/3), DK GRAY BROWN (10YR4/2)

PALE BROWN (10YR6/3), VERY DARK BROWN (10YR2/2), LIGHT REDDISH BROWN (5YR6/4)

15.3

640

18.2

LIGHT/MODERATE WEAR/EROSION ON SURFACE; SOOTING ON EXT. BODY; VESSEL RECONSTRUCTED WITH GLUE, SEVERAL PCS MISSING

SHORT-NECKED GLOBULAR JAR WITH FLARED LIP AND SLIGHTLY FLATTENED BASE

FLATTENED, EVERTED (FLARED)

13.1

1.6

CONCAVE, VERTICAL

DENTATE STAMPED, VERTICAL STAMPING WITH 5-PRONG TOOL

0.5

GLOBULAR WITH SLIGHTLY FLATTENED BASE

18.2

13.7

SMOOTHED; INCISED/TRAILED, CLOSE-SPACED BROAD CONCENTRIC CIRCULAR PATTERN (REPEATED 3X) SURROUNDING ONE HOLLOWED APPLIQUE NODE, DESIGN GOES 3/4 WAY DOWN BODY

UNDISTINGUISHED/SLIGHTLY FLATTENED

3 SINGLE NODES IN CENTERS OF CONCENTRIC CIRCLES ON BODY

Huddleston Grave 1, 11/20/1943; Social Hill-Deceiper phase 
JEC Hodges Collection, 77-1

\begin{tabular}{|c|c|}
\hline Artifact No. / Old Cat. No. / Site No. & $3-22$ / 3CL83 \\
\hline Vessel Form & bottle (sherd lot) \\
\hline ARAS/HSU Digital Photo & K7993 \\
\hline \multicolumn{2}{|l|}{ Type } \\
\hline Decoration & plain \\
\hline \multicolumn{2}{|l|}{ Rim :: Body } \\
\hline Paste & COMPACT, HARD, SMOOTH, ABUN \\
\hline Temper & grog and leached shell, fine, abundant \\
\hline Core Color (Munsell) & VERY DARK GRAY (7.5YR3/1), BRO \\
\hline Exterior Color (Munsell) & BLACK (7.5YR2.5/1), DARK BROWN \\
\hline Interior Color (Munsell) & VERY DARK GRAY (7.5YR3/1), BRO \\
\hline \multicolumn{2}{|l|}{ Vessel Overall Height $(\mathrm{cm})$} \\
\hline Vessel Overall Weight (g) & 531 \\
\hline \multicolumn{2}{|l|}{ Vessel Maximum Diameter $(\mathrm{cm})$} \\
\hline \multicolumn{2}{|l|}{ Volume (liters) } \\
\hline Usewear/Sooting/Condition & Unreconstructed sherd lot of $\sim 70$ sherds \\
\hline Shape/Description & Bottle \\
\hline Lip Treatment/Shape/Angle/Thickness & rounded to beveled ext., sl. everted, 0.3 \\
\hline Orifice Exterior Diameter $(\mathrm{cm})$ & 4.1 \\
\hline Rim/Neck Height $(\mathrm{cm})$ & 7.5 \\
\hline Neck Mid/Base Exterior Diameter $(\mathrm{cm})$ & $4.0 / 4.2$ \\
\hline Rim/Neck Shape/Angle & Straight, slight spool \\
\hline Rim/Neck Surface/Decorative Treatment & Burnished (eroded) \\
\hline Rim/Neck/Body Thickness (cm) & 0.3 \\
\hline \multicolumn{2}{|l|}{ Body Shape } \\
\hline \multicolumn{2}{|l|}{ Body Maximum Diameter $(\mathrm{cm})$} \\
\hline \multicolumn{2}{|l|}{ Body Height $(\mathrm{cm})$} \\
\hline Body Surface/Decorative Treatment & Burnished; Plain \\
\hline Base Shape/Curvature & circular/concave \\
\hline Base Diameter/Height $(\mathrm{cm})$ & 4.8 \\
\hline Base Surface/Decorative Treatment & Smoothed; Plain \\
\hline \multicolumn{2}{|l|}{ Appendages/Handles } \\
\hline Notes & No provenience information \\
\hline
\end{tabular}

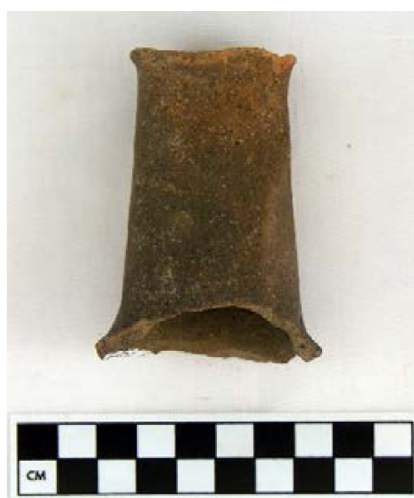

N PASTE 
Artifact No. / Old Cat. No. / Site No.

Vessel Form

ARAS/HSU Digital Photo

Type

Decoration

Rim :: Body

Paste

Temper

Core Color (Munsell)

Exterior Color (Munsell)

Interior Color (Munsell)

Vessel Overall Height (cm)

Vessel Overall Weight $(\mathrm{g})$

Vessel Maximum Diameter (cm)

Volume (liters)

Usewear/Sooting/Condition

Shape/Description

Lip Treatment/Shape/Angle/Thickness Orifice Exterior Diameter $(\mathrm{cm})$

Rim/Neck Height (cm)

Neck Mid/Base Exterior Diameter (cm)

Rim/Neck Shape/Angle

Rim/Neck Surface/Decorative Treatment

Rim/Neck/Body Thickness (cm)

Body Shape

Body Maximum Diameter (cm)

Body Height $(\mathrm{cm})$

Body Surface/Decorative Treatment

Base Shape/Curvature

Base Diameter/Height $(\mathrm{cm})$

Base Surface/Decorative Treatment

Appendages/Handles

Notes
4-1 / 2128 / 3 HS33

bowl, simple

1803

Keno related

incised

plain :: Belhaven 2

SOFT, COMPACT

SHELL (LEACHED) \& GROG

DARK GRAY FIRECLOUDS, REDDISH YELLOW, LIGHT GRAY

VERY DARK GRAY FIRECLOUDS, LIGHT BROWN

5.7

16.3

RING OF ABRASION ON BASE; FIRECLOUDING; VESSEL RECONSTRUCTED IN PAST

SIMPLE OPEN BOWL, RIM CONTINUOUS WITH BODY, SLIGHTLY CONVEX BASE

16.3

STRAIGHT, OUTSLANTED

0.4

CONVEX

16.3

5.7

SMOOTHED; INCISED/TRAILED, NESTED INTERLOCKING SCROLL DESIGN

CIRCULAR, SLIGHTLY CONVEX

6.3

Huddleston Burial X or 1,3/2/1940; Social Hill phase 
JEC Hodges Collection, 77-1

Artifact No. / Old Cat. No. / Site No.

Vessel Form

ARAS/HSU Digital Photo

Type

Decoration

Rim :: Body

Paste

Temper

Core Color (Munsell)

Exterior Color (Munsell)

Interior Color (Munsell)

Vessel Overall Height $(\mathrm{cm})$

Vessel Overall Weight $(\mathrm{g})$

Vessel Maximum Diameter $(\mathrm{cm})$

Volume (liters)

Usewear/Sooting/Condition

Shape/Description

Lip Treatment/Shape/Angle/Thickness Orifice Exterior Diameter $(\mathrm{cm})$

Rim/Neck Height (cm)

Neck Mid/Base Exterior Diameter (cm)

Rim/Neck Shape/Angle

Rim/Neck Surface/Decorative Treatment

Rim/Neck/Body Thickness (cm)

Body Shape

Body Maximum Diameter (cm)

Body Height $(\mathrm{cm})$

Body Surface/Decorative Treatment

Base Shape/Curvature

Base Diameter/Height (cm)

Base Surface/Decorative Treatment

Appendages/Handles

Notes
4-3 / 2476 / 3 HS33

bottle

1821

Keno Trailed

incised

plain :: Belhaven 36 (type)

HARD, COMPACT

SHELL (LEACHED), FINE \& ABUNDANT

WHITE (10YR8/1)

V DK GRAY BROWN (10YR3/2), BROWN (10YR4/3), V PALE BROWN (10YR7/3)

YELLOW (10YR8/6)

16.5

462

15.1

NO WEAR OBSERVED; FIRECLOUDING; RECONSTRUCTED VESSEL, SEVERAL SHERDS MISSING

SMALL, SHORT NECKED, SUB-GLOBULAR BOTTLE WITH PEDESTAL BASE

FLATTENED/THINNED, EVERTED (FLARED)

6.1

4.5

SPOOL

SMOOTHED \& BURNISHED

0.5

SUB-GLOBULAR

15.1

12.0

SMOOTHED \& BURNISHED; INCISED, NESTED SCROLLS

CIRCULAR/PEDESTAL

$8.7 / 1.0$

3 VERTICAL LINES ALT. W/ 1 VERTICAL LINE X4 AROUND PEDESTAL

Huddleston Burial 1A, 3/22 or 3/29/1940; Social Hill - Deceiper phase 
JEC Hodges Collection, 77-1

Artifact No. / Old Cat. No. / Site No.

Vessel Form

ARAS/HSU Digital Photo

Type

Decoration

Rim :: Body

Paste

Temper

Core Color (Munsell)

Exterior Color (Munsell)

Interior Color (Munsell)

Vessel Overall Height (cm)

Vessel Overall Weight (g)

Vessel Maximum Diameter $(\mathrm{cm})$

Volume (liters)

Usewear/Sooting/Condition

Shape/Description

Lip Treatment/Shape/Angle/Thickness Orifice Exterior Diameter $(\mathrm{cm})$

Rim/Neck Height (cm)

Neck Mid/Base Exterior Diameter (cm)

Rim/Neck Shape/Angle

Rim/Neck Surface/Decorative Treatment

Rim/Neck/Body Thickness (cm)

Body Shape

Body Maximum Diameter $(\mathrm{cm})$

Body Height $(\mathrm{cm})$

Body Surface/Decorative Treatment

Base Shape/Curvature

Base Diameter/Height (cm)

Base Surface/Decorative Treatment

Appendages/Handles

Notes
4-4 / 2662 / 3 HS33

bottle

\section{N13162}

Natchitoches/Hodges Engraved

engraved

plain :: Eureka 6 (type)

HARD, SMOOTH, COMPACT

SHELL (LEACHED) \& GROG, FINE \& ABUNDANT

LIGHT GRAY (10YR7/2)

DK GRAY (7.5YR4/1), LT BROWNISH GRAY (10YR6/2), LIGHT GRAY (10YR7/2)

19.0

645

17.4

1.7

some fire clouding present; partial vessel, heavily reconstructed in past; pedestal is hollow, applied after bottle base completed; 2662 inked on pedestal

bottle; short necked, pedestal base; white pigment in engr lines on body (original?)

Burnished, beveled, sl. everted, 0.8

5.6

4.0

4.4 / 4.8

spool

burnished

0.7

low waisted

17.4

12.0

Burnished; engraved, crosshatched/ticked line ovals in 3 tiers w/ diagonal line/balls in between, balls \& crosshatched band below neck

circular/pedestal

$16.7 / 3.0$

Burnished/Plain

Huddleston Burial 4, 10/26/1942; Deceiper phase 
JEC Hodges Collection, 77-1

Artifact No. / Old Cat. No. / Site No.

Vessel Form

ARAS/HSU Digital Photo

Type

Decoration

Rim :: Body

Paste

Temper

Core Color (Munsell)

Exterior Color (Munsell)

Interior Color (Munsell)

Vessel Overall Height $(\mathrm{cm})$

Vessel Overall Weight (g)

Vessel Maximum Diameter $(\mathrm{cm})$

Volume (liters)

Usewear/Sooting/Condition

Shape/Description

Lip Treatment/Shape/Angle/Thickness

Orifice Exterior Diameter $(\mathrm{cm})$

Rim/Neck Height (cm)

Neck Mid/Base Exterior Diameter (cm)

Rim/Neck Shape/Angle

Rim/Neck Surface/Decorative Treatment

Rim/Neck/Body Thickness (cm)

Body Shape

Body Maximum Diameter (cm)

Body Height $(\mathrm{cm})$

Body Surface/Decorative Treatment

Base Shape/Curvature

Base Diameter/Height (cm)

Base Surface/Decorative Treatment

Appendages/Handles

Notes
4-5 / 2645 / 3 HS33

bowl, carinated

K5200

Hodges Engraved var. Nix

Engraved

Esther 12 (sim.) :: plain

COMPACT, HARD, SMOOTH

shell (leached), fine \& abundant + grog

DARK GRAY (7.5YR4/1), LIGHT BROWN (10YR6/3)

DARK GRAY (10YR4/1)

8.5

244

14.4

1.8

Fireclouding; intact vessel; 2645 inked on body

Bowl, Carinated

Burnished, rolled, 0.4

13.3

2.7

Concave, Inslanted

Burnished; engraved, crosshatching, scrolls, balls, ticked line

$0.5-0.3$

Sub-globular

14.4

5.8

Burnished; Plain

undistinguished/convex

Burnished/Plain

Huddleston Burial 2, 9/20/1942; Deceiper phase 


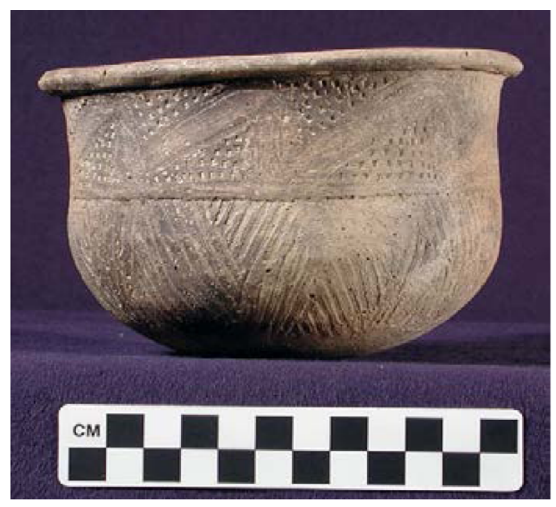

Artifact No. / Old Cat. No. / Site No.

Vessel Form

ARAS/HSU Digital Photo

Type

Decoration

Rim :: Body

Paste

Temper

Core Color (Munsell)

Exterior Color (Munsell)

Interior Color (Munsell)

Vessel Overall Height (cm)

Vessel Overall Weight (g)

Vessel Maximum Diameter $(\mathrm{cm})$

Volume (liters)

Usewear/Sooting/Condition

Shape/Description

Lip Treatment/Shape/Angle/Thickness Orifice Exterior Diameter (cm)

Rim/Neck Height (cm)

Neck Mid/Base Exterior Diameter (cm)

Rim/Neck Shape/Angle

Rim/Neck Surface/Decorative Treatment

Rim/Neck/Body Thickness (cm)

Body Shape

Body Maximum Diameter $(\mathrm{cm})$

Body Height $(\mathrm{cm})$

Body Surface/Decorative Treatment

Base Shape/Curvature

Base Diameter/Height (cm)

Base Surface/Decorative Treatment

Appendages/Handles

Notes
4-6 / 2651 / 3HS33

bowl, simple

1378

Owens Punctated (similar)

incised/punctated

Chatham 10 (type) :: Antioch 1

SOFT, SILTY, COMPACT

SHELL (LEACHED), FINE TO MEDIUM SIZED AND ABUNDANT

REDDISH YELLOW

REDDISH YELLOW

9.2

166

13.7

FIRECLOUDING

BOWL; ROUNDED BODY, STRAIGHT-BEVELED LIP; INCISED AND PUNCTATED DECORATION

ROUNDED/BEVELED, EVERTED (FLARED)

13.7

4.2

STRAIGHT, VERTICAL

SMOOTHED; INCISED \& PUNCTATED, SCROLL DESIGN WITH TOOL PUNCTATES FILLING SPACES

0.3

SUB-GLOBULAR

11.8

5.0

SMOOTHED; INCISED, OPPOSING DIAGONAL LINES

UNDISTINGUISHED/CONVEX

Huddleston Burial 3, 9/26/1942; Deceiper phase 
JEC Hodges Collection, 77-1

Artifact No. / Old Cat. No. / Site No.

Vessel Form

ARAS/HSU Digital Photo

Type

Decoration

Rim :: Body

Paste

Temper

Core Color (Munsell)

Exterior Color (Munsell)

Interior Color (Munsell)

Vessel Overall Height $(\mathrm{cm})$

Vessel Overall Weight $(\mathrm{g})$

Vessel Maximum Diameter $(\mathrm{cm})$

Volume (liters)

Usewear/Sooting/Condition

Shape/Description

Lip Treatment/Shape/Angle/Thickness

Orifice Exterior Diameter $(\mathrm{cm})$

Rim/Neck Height (cm)

Neck Mid/Base Exterior Diameter (cm)

Rim/Neck Shape/Angle

Rim/Neck Surface/Decorative Treatment

Rim/Neck/Body Thickness (cm)

Body Shape

Body Maximum Diameter (cm)

Body Height $(\mathrm{cm})$

Body Surface/Decorative Treatment

Base Shape/Curvature

Base Diameter/Height (cm)

Base Surface/Decorative Treatment

Appendages/Handles

Notes
4-7 / 2778 / 3HS33

bowl, simple

1339

Belcher Engraved var. Belcher

engraved, noded

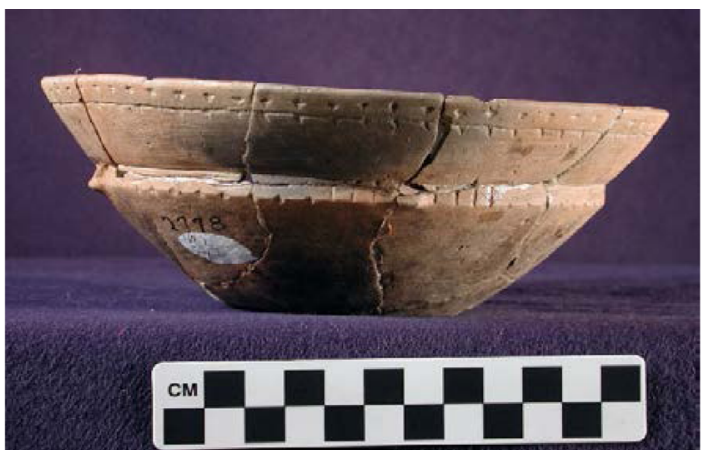

Central $7::$ plain

HARD, COMPACT

GROG (\& SHELL, LEACHED?), FINE \& SPARSE

GRAY (5YR4/2)

DK RED GRAY (5YR4/2), YELLOWISH RED (5YR5/6), V DK GRAY (5YR3/1)

YELLOWISH RED (5YR5/6), VERY DARK GRAY (5YR3/1), REDDISH BROWN (5YR5/3)

7.0

238

17.7

NO USE APPARENT; FIRECLOUDING; VESSEL RECONSTRUCTED WITH GLUE IN PAST, REGLUED, PART OF RIM AND BODY MISSING; 2778 INKED ON SIDE

COMPOUND BOWL-OPEN BOWL, FLAT BASE, DOUBLE RIM WITH ENGRAVED DECORATION

ROUNDED, NONE

17.7

2.7

STRAIGHT, OUTSLANTED UPPER RIM, INSLANTED LOWER RIM

SMOOTHED \& BURNISHED, ENGRAVED TICKED LINE AND JABS ON UPPER RIM, HORIZONTAL LINE, VERTICAL LINES, JABS, NODES ON LOWER RIM

$0.4-0.5$

SHALLOW OPEN COMPOUND BOWL, UPPER RIM CREATED "COLLAR" ON INTERIOR

14.5

4.3

SMOOTHED \& BURNISHED; PLAIN BELOW LOWER RIM

CIRCULAR/FLAT

6.8

4 NODES (1 MISSING) AT LOWER RIM

Huddleston Burial 6, 1/24/1943; Deceiper phase 


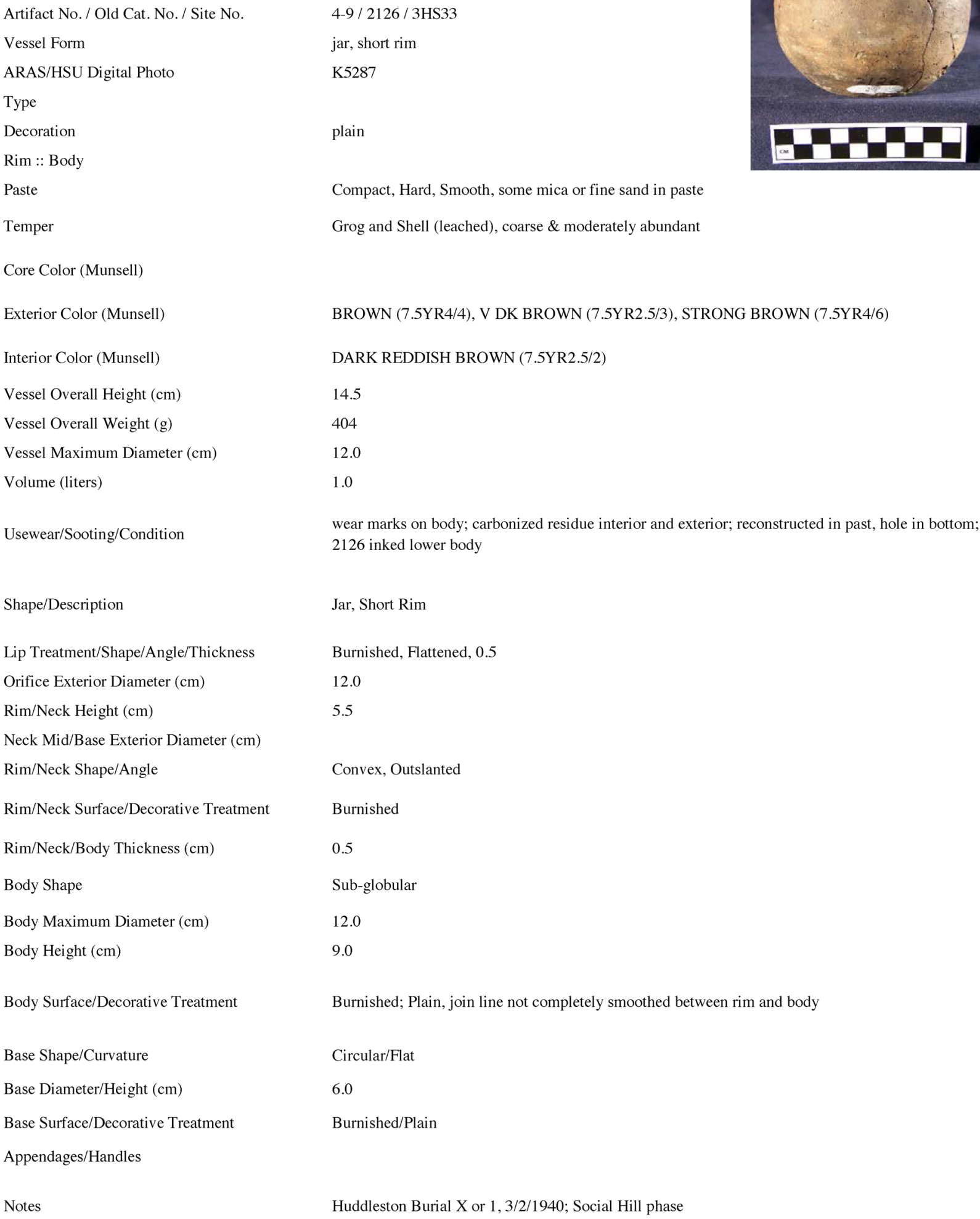

Huddleston Burial X or 1,3/2/1940; Social Hill phase 
Artifact No. / Old Cat. No. / Site No.

Vessel Form

ARAS/HSU Digital Photo

Type

Decoration

Rim :: Body

Paste

Temper

Core Color (Munsell)

Exterior Color (Munsell)

Interior Color (Munsell)

Vessel Overall Height $(\mathrm{cm})$

Vessel Overall Weight (g)

Vessel Maximum Diameter (cm)

Volume (liters)

Usewear/Sooting/Condition

Shape/Description

Lip Treatment/Shape/Angle/Thickness

Orifice Exterior Diameter (cm)

Rim/Neck Height (cm)

Neck Mid/Base Exterior Diameter $(\mathrm{cm})$

Rim/Neck Shape/Angle

Rim/Neck Surface/Decorative Treatment

Rim/Neck/Body Thickness (cm)

Body Shape

Body Maximum Diameter (cm)

Body Height $(\mathrm{cm})$

Body Surface/Decorative Treatment

Base Shape/Curvature

Base Diameter/Height (cm)

Base Surface/Decorative Treatment

Appendages/Handles

Notes
$4-10 / 2666 / 3 \mathrm{HS} 33$

seed jar

N2984

engraved

Elgin (need new) :: Emerson 26

Compact, Hard, Smooth, Mica in paste

Shell (leached) + grog, medium size \& abundant

DARK GRAY (7.5YR4/1), GRAY (7.5YR6/1)

19.7

774

14.8

2.0

Fireclouding; reassembled in past, reconstructed handle; 2666 inked on base

Seed Jar, engraved, four handles

Smoothed, flat, none, 0.4

3.5

1.0

Concave, Inslanted

Smoothed, Engraved, 3 horiz lines 9-10mm apart, 4th spurred line

0.4

Cylindrical

14.8

18.0

Smoothed; Engraved, elongated half circles with reg pigment in engraved lines; lines 6-8mm apart

Circular/Flat

$12.5 / 0.7$

Smoothed/Plain

4 perforated handles ( 2 top, 2 bottom), 1 restored

Huddleston Burial 5, 11/7/1942; Mid-Ouachita phase 
Artifact No. / Old Cat. No. / Site No.

Vessel Form

ARAS/HSU Digital Photo

Type

Decoration

Rim :: Body

Paste

Temper

Core Color (Munsell)

Exterior Color (Munsell)

Interior Color (Munsell)

Vessel Overall Height (cm)

Vessel Overall Weight (g)

Vessel Maximum Diameter $(\mathrm{cm})$

Volume (liters)

Usewear/Sooting/Condition

Shape/Description

Lip Treatment/Shape/Angle/Thickness

Orifice Exterior Diameter $(\mathrm{cm})$

Rim/Neck Height (cm)

Neck Mid/Base Exterior Diameter (cm)

Rim/Neck Shape/Angle

Rim/Neck Surface/Decorative Treatment

Rim/Neck/Body Thickness (cm)

Body Shape

Body Maximum Diameter (cm)

Body Height $(\mathrm{cm})$

Body Surface/Decorative Treatment

Base Shape/Curvature

Base Diameter/Height (cm)

Base Surface/Decorative Treatment

Appendages/Handles

Notes
4-11 / 2125 / 3HS33

bowl, carinated

N3153

Sandford Punctated

punctated

Cedar 3? Caldwell 3 ? :: plain

Compact, Hard, Smooth, Mica in paste

Grog, medium in abundance and size + shell (leached), med, sparse

BLACK (5YR2.5/1), REDDISH BROWN (5YR5/3)

BLACK (5YR2.5/1), REDDISH BROWN (5YR5/3)

7.8

413

17.1

1.0

Bottom roughened (wear); blackened on body/base (soot or fireclouding?); complete vessel, 2 small chips out of rim; 2125 inked on base

Carinated Bowl

Smoothed, Flat/Round, sl. everted, 0.3

15.5

3.5

Convex, Carinated

Smoothed, Punctated, 4 horizontal rows of punctates, triangular tool jabs rather than fingernail

$0.4-0.5$

Convex

17.0

4.3

Burnished; Plain

Undistinguished/Convex

Burnished/Plain

Huddleston Burial X or 1,3/2/1940; Social Hill phase 
JEC Hodges Collection, 77-1

Artifact No. / Old Cat. No. / Site No.

Vessel Form

ARAS/HSU Digital Photo

Type

Decoration

Rim :: Body

Paste

Temper

Core Color (Munsell)

Exterior Color (Munsell)

Interior Color (Munsell)

Vessel Overall Height (cm)

Vessel Overall Weight (g)

Vessel Maximum Diameter $(\mathrm{cm})$

Volume (liters)

Usewear/Sooting/Condition

Shape/Description

Lip Treatment/Shape/Angle/Thickness

Orifice Exterior Diameter $(\mathrm{cm})$

Rim/Neck Height (cm)

Neck Mid/Base Exterior Diameter (cm)

Rim/Neck Shape/Angle

Rim/Neck Surface/Decorative Treatment

Rim/Neck/Body Thickness (cm)

Body Shape

Body Maximum Diameter (cm)

Body Height (cm)

Body Surface/Decorative Treatment

Base Shape/Curvature

Base Diameter/Height $(\mathrm{cm})$

Base Surface/Decorative Treatment

Appendages/Handles

Notes
4-12 / $2667 / 3$ HS33

jar, short rim

N3202

incised/punctated

Butler? Barnard? :: Coker?

Compact, Hard, Smooth

Shell (leached), coarse and abundant

LIGHT REDDISH BROWN (5YR6/4), PINK (7.5YR7/4)

LT BROWN (7.5YR6/4), DK GRAY (7.5YR4/1), LT RED BROWN (5YR6/4)

LIGHT BROWN (7.5YR6/4), DARK GRAY (7.5YR4/1)

17.0

545

22.0

Some sooting on exterior shoulder, fireclouding; partial vessel, reconstructed with plaster in past; 2667 inked near base

Jar, Short Rim

Smoothed, rounded, everted, 0.4

16.0

2.5

Convex, Inslanted

Smoothed, Incised, 8 horizontal incised lines- close spaced wide lines, $2 \mathrm{~mm}$

0.5

High-waisted

22.0

14.5

Smoothed; Punctated, four rows of horizontal fingernail? punctations around shoulder/top of body

Undistinguished/Convex

Smoothed/Plain

Huddleston Burial 5, 11/7/1942; Mid-Ouachita phase 
JEC Hodges Collection, 77-1

Artifact No. / Old Cat. No. / Site No.

Vessel Form

ARAS/HSU Digital Photo

Type

Decoration

Rim :: Body

Paste

Temper

Core Color (Munsell)

Exterior Color (Munsell)

Interior Color (Munsell)

Vessel Overall Height (cm)

Vessel Overall Weight (g)

Vessel Maximum Diameter (cm)

Volume (liters)

Usewear/Sooting/Condition

Shape/Description

Lip Treatment/Shape/Angle/Thickness

Orifice Exterior Diameter (cm)

Rim/Neck Height (cm)

Neck Mid/Base Exterior Diameter (cm)

Rim/Neck Shape/Angle

Rim/Neck Surface/Decorative Treatment

Rim/Neck/Body Thickness (cm)

Body Shape

Body Maximum Diameter $(\mathrm{cm})$

Body Height $(\mathrm{cm})$

Body Surface/Decorative Treatment

Base Shape/Curvature

Base Diameter/Height $(\mathrm{cm})$

Base Surface/Decorative Treatment

Appendages/Handles

Notes
4-13 / 2665 / 3HS33

bowl, carinated

$\mathrm{N} 4442$

Friendship Engraved var. Freeman

engraved

Elaine 2 (type) :: plain

Compact, Hard, Smooth

Shell (leached) and Grog, fine and abundant

BROWN (10YR5/3), DARK GRAY (10YR4/1), DARK REDDISH GRAY (2.5YR3/1)

VERY DARK GRAY (10YR3/1)

9.5

615

22.6

2.4

Fireclouding; Intact vessel; 2665 inked on base

Carinated Bowl

Burnished, rounded, everted, 0.4

21.7

4.0

Straight, Carinated/Inslanted

Burnished, Engraved, three tiers, horizontal ovals, crosshatching, ticked lines

$0.5-0.7$

Convex

22.6

4.3

Burnished; Plain

Undistinguished/Convex

Burnished/Plain

Huddleston Burial 5, 11/7/1942; Mid-Ouachita phase 
Artifact No. / Old Cat. No. / Site No.

Vessel Form

ARAS/HSU Digital Photo

Type

Decoration

Rim :: Body

Paste

Temper

Core Color (Munsell)

Exterior Color (Munsell)

Interior Color (Munsell)

Vessel Overall Height $(\mathrm{cm})$

Vessel Overall Weight (g)

Vessel Maximum Diameter $(\mathrm{cm})$

Volume (liters)

Usewear/Sooting/Condition

Shape/Description

Lip Treatment/Shape/Angle/Thickness

Orifice Exterior Diameter $(\mathrm{cm})$

Rim/Neck Height (cm)

Neck Mid/Base Exterior Diameter $(\mathrm{cm})$

Rim/Neck Shape/Angle

Rim/Neck Surface/Decorative Treatment

Rim/Neck/Body Thickness (cm)

Body Shape

Body Maximum Diameter $(\mathrm{cm})$

Body Height (cm)

Body Surface/Decorative Treatment

Base Shape/Curvature

Base Diameter/Height $(\mathrm{cm})$

Base Surface/Decorative Treatment

Appendages/Handles

Notes
4-14 / 2777 / 3HS33

bottle

N3645

Keno Trailed

trailed, punctated

Cornell 3 and Caldwell 5 :: Belhaven 17

Compact, Hard, Smooth, Abundant Mica

Grog, fine \& sparse+ shell (leached), very sparse (voids)

YELLOWISH RED (5YR5/6)

GRAY (7.5YR5/1), LIGHT REDDISH BROWN (5YR6/4), PINK (5YR8/4)

GRAY (7.5YR5/1)

14.0

473

13.4

0.7

Fireclouding; vessel reconstructed in past, missing small sherd from rim, lip chipped; 2777 inked on base

Bottle, short neck, pedestal

Burnished, rounded, everted, 0.4 ; thin incised notches on top of lip, perpendicular 5.9

2.8

$5.1 / 5.2$

Spool

Burnished, Punctated, three rows of vertical jabs

0.4

Low waisted

13.4

8.7

Burnished; Incised/Trailed, lines $2 \mathrm{~mm}$ wide, $5 \mathrm{~mm}$ apart nested arcs (Vs over Us) X4 around body

Circular/Pedestal

$7.6 / 2.5$

Burnished; incised/trailed lines w. punctated panels between, X3 around pedestal

Huddleston Burial 6, 1/24/1943; Deceiper phase 
Artifact No. / Old Cat. No. / Site No.

Vessel Form

ARAS/HSU Digital Photo

Type

Decoration

$\operatorname{Rim}::$ Body

Paste

Temper

Core Color (Munsell)

Exterior Color (Munsell)

Interior Color (Munsell)

Vessel Overall Height (cm)

Vessel Overall Weight (g)

Vessel Maximum Diameter $(\mathrm{cm})$

Volume (liters)

Usewear/Sooting/Condition

Shape/Description

Lip Treatment/Shape/Angle/Thickness

Orifice Exterior Diameter $(\mathrm{cm})$

Rim/Neck Height (cm)

Neck Mid/Base Exterior Diameter (cm)

Rim/Neck Shape/Angle

Rim/Neck Surface/Decorative Treatment

Rim/Neck/Body Thickness (cm)

Body Shape

Body Maximum Diameter $(\mathrm{cm})$

Body Height $(\mathrm{cm})$

Body Surface/Decorative Treatment

Base Shape/Curvature

Base Diameter/Height (cm)

Base Surface/Decorative Treatment

Appendages/Handles
4-15 / $2661 / 3$ HS33

bowl, simple

4846, N4611

Hodges Engraved var. Hodges

Engraved

Elgin 2 :: Ebbet 6

Compact, Hard, Smooth

Shell (leached, fine \& abundant) + Grog (fine \& sparse)

DARK GRAY (7.5YR4/1), BROWN (7.5YR5/2), YELLOWISH RED (5YR5/6)

LIGHT BROWN (7.5YR6/3), DARK GRAY (7.5YR4/1)

8.8

286

16.2

0.8

Fireclouding; intact vessel; 2661 inked on base

Bowl (cup)

Burnished, beveled, sl. everted, 0.5

16.2

straight, outslanted (continuous with body)

Burnished; Engraved, 2 horizontal ticked lines below lip

0.4

Convex

14.8

8.8

Burnished; Engraved, ticked line hook elements up and down, bkgd crosshatched \& balls, X2, 2 horiz ticked lines at base; lacks extra U shaped elements of other Ebbet 6

Circular/Convex

8.0

Burnished/Plain

Huddleston Burial 4, 10/26/1942; Deceiper phase 
Artifact No. / Old Cat. No. / Site No.

Vessel Form

ARAS/HSU Digital Photo

Type

Decoration

Rim :: Body

Paste

Temper

Core Color (Munsell)

Exterior Color (Munsell)

Interior Color (Munsell)

Vessel Overall Height $(\mathrm{cm})$

Vessel Overall Weight (g)

Vessel Maximum Diameter (cm)

Volume (liters)

Usewear/Sooting/Condition

Shape/Description

Lip Treatment/Shape/Angle/Thickness

Orifice Exterior Diameter (cm)

Rim/Neck Height (cm)

Neck Mid/Base Exterior Diameter (cm)

Rim/Neck Shape/Angle

Rim/Neck Surface/Decorative Treatment

Rim/Neck/Body Thickness (cm)

Body Shape

Body Maximum Diameter $(\mathrm{cm})$

Body Height (cm)

Body Surface/Decorative Treatment

Base Shape/Curvature

Base Diameter/Height $(\mathrm{cm})$

Base Surface/Decorative Treatment

Appendages/Handles

Notes
4-16 / 2644 / 3 HS33

jar, short rim

N4813

De Roche Incised

incised/punctated

Caldwell 7 :: Antioch 17

Compact, Hard, Smooth

Shell (leached), medium size \& abundant

PINKISH GRAY (7.5YR6/2)

BLACK (7.5YR2.5/1), DARK GRAY (7.5YR4/1)

BLACK (7.5YR2.5/1), DARK GRAY (7.5YR4/1), BROWN (7.5YR5/2)

11.0

190

12.5

sooting on ext/int near rim; partial vessel, reconstructed in past \& reglued, missing base and sev body sherds; no inked number

Jar

Smoothed, rounded, sl. everted, 0.2

11.0

1.0

Convex, Outslanted

Smoothed; Punctated, 1 line of tool punctates (small, rounded) near rim/body junction

$0.5-0.4$

Subglobular

12.5

10.0

Smoothed; Incised, close-spaced lines, zoned line-filled triangles and diamonds

missing; from sherds, slightly flattened

plain

Huddleston Burial 2, 9/20/1942; Deceiper phase 
JEC Hodges Collection, 77-1

Artifact No. / Old Cat. No. / Site No.

Vessel Form

ARAS/HSU Digital Photo

Type

Decoration

$\operatorname{Rim}::$ Body

Paste

Temper

Core Color (Munsell)

Exterior Color (Munsell)

Interior Color (Munsell)

Vessel Overall Height (cm)

Vessel Overall Weight (g)

Vessel Maximum Diameter (cm)

Volume (liters)

Usewear/Sooting/Condition

Shape/Description

Lip Treatment/Shape/Angle/Thickness

Orifice Exterior Diameter $(\mathrm{cm})$

Rim/Neck Height (cm)

Neck Mid/Base Exterior Diameter (cm)

Rim/Neck Shape/Angle

Rim/Neck Surface/Decorative Treatment

Rim/Neck/Body Thickness (cm)

Body Shape

Body Maximum Diameter (cm)

Body Height (cm)

Body Surface/Decorative Treatment

Base Shape/Curvature

Base Diameter/Height (cm)

Base Surface/Decorative Treatment

Appendages/Handles

Notes
4-17/2650 / 3HS33

jar, short rim

K5232

De Roche Incised

incised/punctated

Caldwell 7 :: Adrian 1

Compact, Hard, Smooth

Shell (leached), coarse \& abundant

PINK (7.5YR7/4), DARK GRAY (7.5YR4/1), BROWN (7.5YR5/2)

PINK (7.5YR7/4), BROWN (7.5YR5/2)

8.5

148

12.0

0.6

partial vessel, reconstructed in past, hole in side

Jar, Short Rim

Smoothed, rounded, everted (flared), 0.4

11.0

1.0

Concave, vertical

Smoothed, Punctated, 1 row of vertical tool punctations

$0.4-0.5$

Sub-Globular

12.0

7.5

Smoothed; incised, broad incised/trailed nested diamond pattern covering body

Undistinguished/Convex

Smoothed/Plain

Huddleston Burial 3, 9/26/1942; Deceiper phase 
JEC Hodges Collection, 77-1

Artifact No. / Old Cat. No. / Site No.

Vessel Form

ARAS/HSU Digital Photo

Type

Decoration

Rim :: Body

Paste

Temper

Core Color (Munsell)

Exterior Color (Munsell)

Interior Color (Munsell)

Vessel Overall Height (cm)

Vessel Overall Weight (g)

Vessel Maximum Diameter (cm)

Volume (liters)

Usewear/Sooting/Condition

Shape/Description

Lip Treatment/Shape/Angle/Thickness

Orifice Exterior Diameter (cm)

Rim/Neck Height (cm)

Neck Mid/Base Exterior Diameter (cm)

Rim/Neck Shape/Angle

Rim/Neck Surface/Decorative Treatment

Rim/Neck/Body Thickness (cm)

Body Shape

Body Maximum Diameter $(\mathrm{cm})$

Body Height (cm)

Body Surface/Decorative Treatment

Base Shape/Curvature

Base Diameter/Height (cm)

Base Surface/Decorative Treatment

Appendages/Handles

Notes
$5-1 / 1241 / 3 C L 81$

bowl, simple

1801

Keno related

incised

plain :: Belhaven 2

SOFT, CRUMBLY

SHELL (LEACHED), FINE TO MEDIUM, ABUNDANT

DARK GRAY

BROWN, VERY DARK GRAY FIRECLOUDS, PINKISH GRAY

GRAY, VERY DARK GRAY

10.3

16.6

SURFACE ERODED, BASE ABRADED; POSSIBLE TRACES OF SOOTING ON EXT, FIRECLOUDING; VESSEL RECONSTRUCTED IN PAST

SIMPLE GLOBULAR BOWL WITH CONVEX BASE, SHORT FLARING RIM

ROUNDED, EVERTED (FLARED)

16.6

1.9

STRAIGHT, OUTSLANTED

SMOOTHED

$0.4-0.3$

SUB-GLOBULAR

14.5

8.4

SMOOTHED; INCISED/TRAILED, SCROLLS, VERY DIFFICULT TO DISCERN TOTAL PATTERN

UNDISTINGUISHED/CONVEX

4.3

SMOOTHED/PLAIN

Huddleston 'from Stanford mound', Phillips photo 3889 (Huddleston 213) 
Artifact No. / Old Cat. No. / Site No.

Vessel Form

ARAS/HSU Digital Photo

Type

Decoration

Rim :: Body

Paste

Temper

Core Color (Munsell)

Exterior Color (Munsell)

Interior Color (Munsell)

Vessel Overall Height $(\mathrm{cm})$

Vessel Overall Weight (g)

Vessel Maximum Diameter (cm)

Volume (liters)

Usewear/Sooting/Condition

Shape/Description

Lip Treatment/Shape/Angle/Thickness

Orifice Exterior Diameter (cm)

Rim/Neck Height (cm)

Neck Mid/Base Exterior Diameter (cm)

Rim/Neck Shape/Angle

Rim/Neck Surface/Decorative Treatment

Rim/Neck/Body Thickness (cm)

Body Shape

Body Maximum Diameter (cm)

Body Height $(\mathrm{cm})$

Body Surface/Decorative Treatment

Base Shape/Curvature

Base Diameter/Height (cm)

Base Surface/Decorative Treatment

Appendages/Handles

Notes
$5-2 / 1239 / 3$ CL81

bottle

689

Keno Trailed

incised

plain :: Belhaven 1, 18 (sim.)

SOFT, SILTY, COMPACT

GROG, FINE \& GRIT

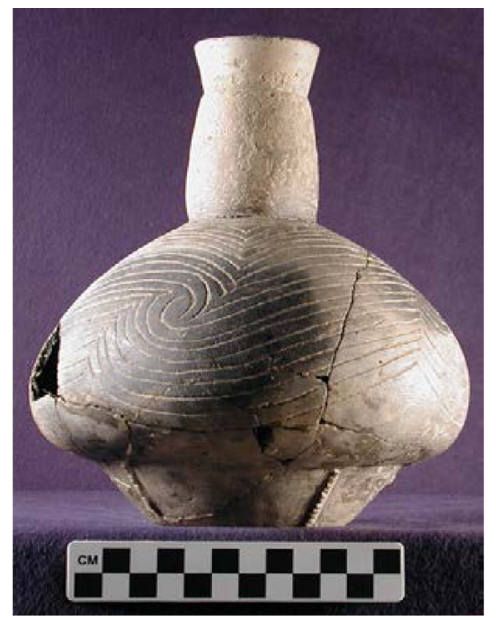

GRAY (10YR5/1)

V PALE BROWN (10YR7/4), DK GRAY (10YR4/1), RED (2.5YR4/8) INCISING

LIGHT BROWNISH GRAY (10YR6/2)

22.5

17.2

SURFACE ERODED; FIRECLOUDING; VESSEL RECONSTRUCTED, BODY SHERD MISSING, BOTTLE NECK NOT ATTACHED

BOTTLE, GLOBULAR-LONG NECKED-PEDESTAL BASE, FLARED SPOOL NECK

ROUNDED, SL. THINNED, EVERTED (FLARED)

5.0

2.2

BULBOUS / SPOOL

SMOOTHED, PLAIN; ERODED

$0.4-0.5$

LOW-WAISTED

17.2

14.3

SMOOTHED \& BURNISHED; INCISED ARCS AND INTERLOCKING SCROLLS, 3 HORIZ LINES AT BASE OF NECK; TRACE RED PIGMENT IN INCISED [ENGRAVED?] LINES

CIRCULAR/PEDESTAL

$12.1 / 4.0$

SMOOTHED/BURNISHED; 4 NOTCHED VERTICAL APPLIQUE STRIPS

APPLIQUE STRIPS ON PEDISTAL

Huddleston Burial 11, 5/20/1938; Phillips photo 3896 (Huddleston 57); Social Hill phase 
JEC Hodges Collection, 77-1

Artifact No. / Old Cat. No. / Site No.

Vessel Form

ARAS/HSU Digital Photo

Type

Decoration

Rim :: Body

Paste

Temper

Core Color (Munsell)

Exterior Color (Munsell)

Interior Color (Munsell)

Vessel Overall Height $(\mathrm{cm})$

Vessel Overall Weight (g)

Vessel Maximum Diameter (cm)

Volume (liters)

Usewear/Sooting/Condition

Shape/Description

Lip Treatment/Shape/Angle/Thickness

Orifice Exterior Diameter (cm)

Rim/Neck Height (cm)

Neck Mid/Base Exterior Diameter (cm)

Rim/Neck Shape/Angle

Rim/Neck Surface/Decorative Treatment

Rim/Neck/Body Thickness (cm)

Body Shape

Body Maximum Diameter $(\mathrm{cm})$

Body Height (cm)

Body Surface/Decorative Treatment

Base Shape/Curvature

Base Diameter/Height (cm)

Base Surface/Decorative Treatment

Appendages/Handles

Notes
5-3 / $1392 / 3$ CL81

bottle

1149

Keno Trailed

incised

plain :: Belhaven 1

MEDIUM-HARD, COMPACT

SHELL (SOME LEACHED), MEDIUM SIZE AND ABUNDANT

$\operatorname{BLACK}(10 \mathrm{YR} 2 / 1)$

BLACK (10YR2/1), VERY DARK GRAY (10YR3/1), BROWN (10YR5/3)

19.2

458

13.7

MINIMAL WEAR ON BASE, LIP CHIPPING PROBABLY POST-DEPOSITIONAL; FIRECLOUDING; COMPLETE VESSEL, NECK GLUED TO BODY IN PAST; RESIDUE OF PAPER TAG VISIBLE NEAR BASE

BOTTLE-LONG NECK WITH FLARED LIP-SQUAT GLOBULAR BODY-FLAT CIRCULAR BASE-BODY HAS INCISED DECORATION

BEVELED, EVERTED (FLARED)

4.8

8.4

BULBOUS / SPOOL

SMOOTHED

0.5

LOW-WAISTED

13.7

10.8

SMOOTHED \& BURNISHED; INCISED LINE DECORATION ON BODY-SCROLL REPEATED 4X-LINES 2-3MM WIDE, 5-7MM APART

CIRCULAR/FLAT

5.5

Huddleston Burial 26, 3/25/1939; Phillips photo 3892 (Huddleston 173); Social Hill-Deceiper phase 
Artifact No. / Old Cat. No. / Site No.

Vessel Form

ARAS/HSU Digital Photo

Type

Decoration

Rim :: Body

Paste

Temper

Core Color (Munsell)

Exterior Color (Munsell)

Interior Color (Munsell)

Vessel Overall Height $(\mathrm{cm})$

Vessel Overall Weight (g)

Vessel Maximum Diameter $(\mathrm{cm})$

Volume (liters)

Usewear/Sooting/Condition

Shape/Description

Lip Treatment/Shape/Angle/Thickness

Orifice Exterior Diameter (cm)

Rim/Neck Height $(\mathrm{cm})$

Neck Mid/Base Exterior Diameter (cm)

Rim/Neck Shape/Angle

Rim/Neck Surface/Decorative Treatment

Rim/Neck/Body Thickness (cm)

Body Shape

Body Maximum Diameter $(\mathrm{cm})$

Body Height (cm)

Body Surface/Decorative Treatment

Base Shape/Curvature

Base Diameter/Height (cm)

Base Surface/Decorative Treatment

Appendages/Handles

Notes
$5-4 / 1242$ / 3CL81

bowl, carinated

3922

Cook Engraved

engraved

Eddy 3 :: plain

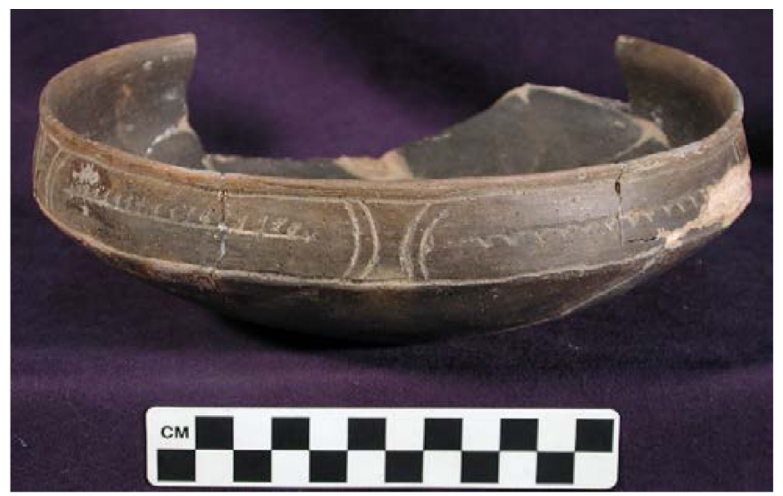

HARD, SILTY, COMPACT

SHELL (SOME LEACHED), FINE

DARK GRAY (7.5YR4/1)

REDDISH YELLOW (7.5YR6/6), BLACK (10YR2/1), GRAYISH BROWN (10YR5/2)

REDDISH YELLOW (7.5YR6/6), BLACK (10YR2/1), GRAYISH BROWN (10YR5/2)

7.9

408

20.0

WEAR/EROSION/LEACHING IN SEVERAL AREAS; FIRECLOUDING; VESSEL RECONSTRUCTED WITH GLUE IN PAST, SEVERAL FRAGMENTS MISSING/UNATTACHED; 1242 INKED ON BASE, REMNANT PAPER TAG INT.

CARINATED BOWL WITH INCURVED RIM, FLARED AND FLATTENED LIP, BROAD USHAPE BODY, AND ROUND BASE

ROUNDED, SL. EVERTED (FLARED)

19.4

2.9

STRAIGHT, INSLANTED/CARINATED

SMOOTHED \& BURNISHED; ENGRAVED, PANELS OF ENGRAVED LINES, TICKED LINES W TICKING ALTERNATING UP/DOWN

0.5

CONVEX

20.0

5.0

SMOOTHED \& BURNISHED; PLAIN

UNDISTINGUISHED/CONVEX

Huddleston Burial 17, 6/11/1938; Phillips photo 3902 (Huddleston 66); Social Hill phase 
JEC Hodges Collection, 77-1

Artifact No. / Old Cat. No. / Site No.

Vessel Form

ARAS/HSU Digital Photo

Type

Decoration

Rim :: Body

Paste

Temper

Core Color (Munsell)

Exterior Color (Munsell)

Interior Color (Munsell)

Vessel Overall Height $(\mathrm{cm})$

Vessel Overall Weight (g)

Vessel Maximum Diameter (cm)

Volume (liters)

Usewear/Sooting/Condition

Shape/Description

Lip Treatment/Shape/Angle/Thickness

Orifice Exterior Diameter $(\mathrm{cm})$

Rim/Neck Height (cm)

Neck Mid/Base Exterior Diameter $(\mathrm{cm})$

Rim/Neck Shape/Angle

Rim/Neck Surface/Decorative Treatment

Rim/Neck/Body Thickness (cm)

Body Shape

Body Maximum Diameter (cm)

Body Height (cm)

Body Surface/Decorative Treatment

Base Shape/Curvature

Base Diameter/Height $(\mathrm{cm})$

Base Surface/Decorative Treatment

Appendages/Handles

Notes

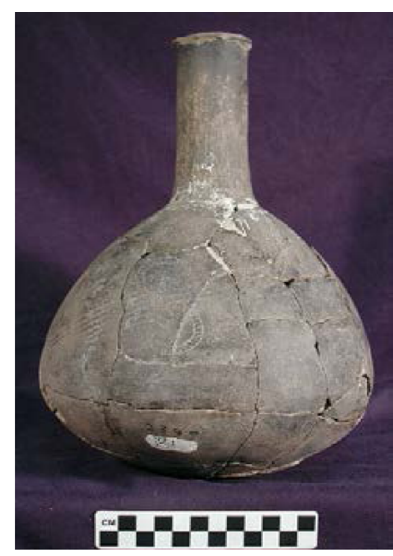

5-5 / 2397 / 3CL81

bottle

3928

Blakely Engraved var. Blakely

engraved

plain :: Esop 3

HARD, SILTY, COMPACT

GROG, FINE \& ABUNDANT + SHELL (LEACHED), FINE \& SPARSE

GRAYISH BROWN (10YR5/2)

GRAY (7.5YR5/1), DARK GRAY (7.5YR4/1), GRAYISH BROWN (10YR5/2)

GRAY (10YR5/1), PALE BROWN (10YR6/3), VERY PALE BROWN (10YR7/4)

29.8

964

20.5

BASE MODERATELY/HEAVILY WORN; SURFACE WEAR/EROSION ON BODY; VESSEL RECONSTRUCTED WITH GLUE AND PLASTER IN PAST, SEVERAL MISSING SHERDS; 2397 INKED NEAR BASE

LARGE LONG-NECK BOTTLE WITH FLARED AND ROUNDED LIP, GLOBULAR BODY, AND ROUNDED BASE

ROUNDED, SL. EVERTED (FLARED)

5.5

11.3

VERTICAL, SLIGHT SPOOL

SMOOTHED \& BURNISHED (ERODED), PLAIN

$0.6-0.5$

LOW-WAISTED

20.5

18.5

SMOOTHED \& BURNISHED (ERODED); ENGRAVED, DIAGONAL LINE UNIT WITH CURVILINEAR FEATURES AT CENTRAL ELEMENT

UNDISTINGUISHED/CONVEX

Huddleston Burial 26 (27?), 1/4/1940 or 1/4/1941; Mid-Ouachita-Social Hill phase; publ. Trubitt 2017 
Artifact No. / Old Cat. No. / Site No.

Vessel Form

ARAS/HSU Digital Photo

Type

Decoration

Rim :: Body

Paste

Temper

Core Color (Munsell)

Exterior Color (Munsell)

Interior Color (Munsell)

Vessel Overall Height (cm)

Vessel Overall Weight (g)

Vessel Maximum Diameter (cm)

Volume (liters)

Usewear/Sooting/Condition

Shape/Description

Lip Treatment/Shape/Angle/Thickness

Orifice Exterior Diameter $(\mathrm{cm})$

Rim/Neck Height (cm)

Neck Mid/Base Exterior Diameter (cm)

Rim/Neck Shape/Angle

Rim/Neck Surface/Decorative Treatment

Rim/Neck/Body Thickness (cm)

Body Shape

Body Maximum Diameter (cm)

Body Height $(\mathrm{cm})$

Body Surface/Decorative Treatment

Base Shape/Curvature

Base Diameter/Height (cm)

Base Surface/Decorative Treatment

Appendages/Handles

Notes
5-6 / 1437 / 3CL81

jar, tall rim

3924

Caney Punctated variant?

brushed/ridge pinched

Dana 4 :: Curry 4 (type)

SOFT, GRANULAR, CRUMBLY

SHELL (SOME LEACHED), COARSE \& ABUNDANT

$\operatorname{BLACK}(5 Y R 2 / 1)$

DK GRAY (10YR4/1), V DK GRAY (7.5YR3/1), REDDISH YELLOW (5YR6/6)

VERY DARK GRAY (7.5YR3/1), DARK GRAY (5YR4/1), DARK GRAYISH BROWN (10YR4/2)

12.8

287

15.3

WEAR/SURFACE EROSION/LEACHING; BLACKENED INT., FIRECLOUDING; PARTIAL VESSEL, RECONSTRUCTED WITH GLUE, MOST OF RIM IS MISSING/UNATTACHED

SMALL TALL-RIM JAR WITH A FLARED AND ROUNDED LIP, GLOBULAR BODY, AND FLATTENED BASE

ROUNDED, SL. EVERTED (FLARED)

15.3

4.8

STRAIGHT, OUTSLANTED

SMOOTHED; BRUSHED, IRREG HORIZONTAL BRUSHING AROUND RIM

$0.5-0.4$

SUB-GLOBULAR

11.7

8.0

SMOOTHED; RIDGE PINCHED, PAIRED COLUMNS OF PUNCTATES CREATE VERTICAL RIDGES (REPEATED $4 \mathrm{X}$ ), PATTERN SLOPES DIAGONALLY FROM LEFT TO RIGHT

CIRCULAR, FLAT

6.0

Huddleston Burial 17, 6/11/1938; Phillips photo 3916 (Huddleston 68); Social Hill phase 
JEC Hodges Collection, 77-1

Artifact No. / Old Cat. No. / Site No.

Vessel Form

ARAS/HSU Digital Photo

Type

Decoration

Rim :: Body

Paste

Temper

Core Color (Munsell)

Exterior Color (Munsell)

Interior Color (Munsell)

Vessel Overall Height $(\mathrm{cm})$

Vessel Overall Weight (g)

Vessel Maximum Diameter (cm)

Volume (liters)

Usewear/Sooting/Condition

Shape/Description

Lip Treatment/Shape/Angle/Thickness

Orifice Exterior Diameter $(\mathrm{cm})$

Rim/Neck Height (cm)

Neck Mid/Base Exterior Diameter (cm)

Rim/Neck Shape/Angle

Rim/Neck Surface/Decorative Treatment

Rim/Neck/Body Thickness (cm)

Body Shape

Body Maximum Diameter $(\mathrm{cm})$

Body Height $(\mathrm{cm})$

Body Surface/Decorative Treatment

Base Shape/Curvature

Base Diameter/Height $(\mathrm{cm})$

Base Surface/Decorative Treatment

Appendages/Handles

Notes
$5-7 / 1282 / 3$ CL81

jar, short rim

3926

Karnack Brushed-Incised?

incised

Austin $1::$ Abraham 3 (type)

SOFT, GRANULAR, CRUMBLY

SHELL (LEACHED), COARSE \& ABUNDANT + BONE, FINE \& SPARSE

VERY DARK GRAY (10YR3/1)

DARK GRAY (10YR4/1), BLACK (7.5YR2.5/1), STRONG BROWN (7.5YR5/6)

DARK GRAY (10YR4/1), VERY DARK GRAY (10YR3/1), REDDISH YELLOW (5YR6/6)

14.0

305

13.0

WORN/ERODED/LEACHED SURFACES; BLACKENED INT. AND UPPER BODY EXT.; VESSEL RECONSTRUCTED WITH GLUE, PART OF RIM MISSING

SMALL SHORT-RIM JAR WITH FLARED AND ROUNDED LIP, TALL AND RAIRLY STRAIGHT-SIDED BODY, AND FLAT BASE

ROUNDED, EVERTED (FLARED)

13.0

1.0

CONCAVE, OUTSLANTED

SMOOTHED; INCISED, SHORT VERTICAL INCISED LINES

$0.4-0.5$

ELONGATED

11.5

13.0

SMOOHTED; INCISED, MEDIUM-WIDE SPACED VERTICAL SHALLOW INCISED LINES AROUND VESSEL

CIRCULAR, FLAT

6.0

Huddleston Burial 26, 3/25/1939; Phillips photo 3884 (Huddleston 170); Social Hill-Deceiper phase 
Artifact No. / Old Cat. No. / Site No.

Vessel Form

ARAS/HSU Digital Photo

Type

Decoration

Rim :: Body

Paste

Temper

Core Color (Munsell)

Exterior Color (Munsell)

Interior Color (Munsell)

Vessel Overall Height (cm)

Vessel Overall Weight (g)

Vessel Maximum Diameter (cm)

Volume (liters)

Usewear/Sooting/Condition

Shape/Description

Lip Treatment/Shape/Angle/Thickness

Orifice Exterior Diameter $(\mathrm{cm})$

Rim/Neck Height (cm)

Neck Mid/Base Exterior Diameter (cm)

Rim/Neck Shape/Angle

Rim/Neck Surface/Decorative Treatment

Rim/Neck/Body Thickness (cm)

Body Shape

Body Maximum Diameter $(\mathrm{cm})$

Body Height $(\mathrm{cm})$

Body Surface/Decorative Treatment

Base Shape/Curvature

Base Diameter/Height (cm)

Base Surface/Decorative Treatment

Appendages/Handles

Notes
5-8 / 1314 / 3CL81

bottle

3932

plain

HARD, SILTY, COMPACT

SHELL (SOME LEACHED), FINE, MODERATELY ABUND.

REDDISH BROWN (5YR5/4), GRAY (10YR5/1)

BLACK (10YR2/1), YELLOWISH BROWN (10YR5/4), V DK GRAY (10YR3/1)

DARK GRAY (5YR4/1), VERY DARK GRAY (10YR3/1)

18.8

559

14.1

MINOR WEAR AROUND BASE RING; FIRECLOUDING; PARTIAL VESSEL, RECONSTRUCTED WITH GLUE IN PAST, 1/3 OF NECK MISSING

MEDIUM-TALL NECKED BOTTLE WITH FLARED AND ROUNDED, SQUAT-GLOBULAR BODY, AND FLAT AND CONCAVE BASE

ROUNDED, SL. EVERTED (FLARED)

4.0 (est.)

7.7

BULBOUS / SPOOL

SMOOTHED \& BURNISHED, PLAIN

0.4

LOW-WAISTED

14.1

11.1

SMOOTHED \& BURNISHED; PLAIN

CIRCULAR, FLAT/CONCAVE

4.5

Huddleston Burial 26, 3/25/1939; Phillips photo 3880 (Huddleston 169); Social Hill-Deceiper phase 
Artifact No. / Old Cat. No. / Site No.

Vessel Form

ARAS/HSU Digital Photo

Type

Decoration

$\operatorname{Rim}::$ Body

Paste

Temper

Core Color (Munsell)

Exterior Color (Munsell)

Interior Color (Munsell)

Vessel Overall Height $(\mathrm{cm})$

Vessel Overall Weight (g)

Vessel Maximum Diameter $(\mathrm{cm})$

Volume (liters)

Usewear/Sooting/Condition

Shape/Description

Lip Treatment/Shape/Angle/Thickness

Orifice Exterior Diameter $(\mathrm{cm})$

Rim/Neck Height (cm)

Neck Mid/Base Exterior Diameter (cm)

Rim/Neck Shape/Angle

Rim/Neck Surface/Decorative Treatment

Rim/Neck/Body Thickness (cm)

Body Shape

Body Maximum Diameter (cm)

Body Height (cm)

Body Surface/Decorative Treatment

Base Shape/Curvature

Base Diameter/Height $(\mathrm{cm})$

Base Surface/Decorative Treatment

Appendages/Handles

Notes

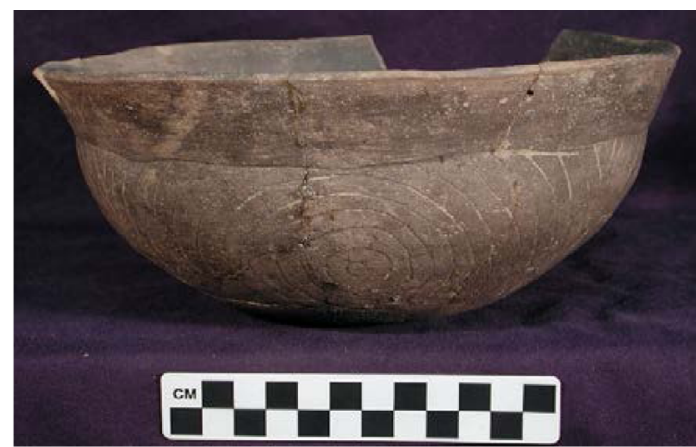

engraved

plain :: Evansville 4

HARD, FINE, SILTY, COMPACT

SHELL (SOME LEACHED) \& BONE, FINE \& MODERATELY ABUND.

GRAY $(7.5 Y R 5 / 1)$

DK GRAY (10YR4/1), BLACK (7.5YR2.5/1), LT BROWNISH GRAY (10YR6/2)

VERY DARK GRAY (7.5YR3/1), DARK GRAY (10YR4/1), LIGHT BROWNISH GRAY (10YR6/2)

10.6

346

21.1

BASE IS MODERATELY WORN; BLACKENED INT., FIRECLOUSING; VESSEL

RECONSTRUCTED WITH GLUE IN PAST, SEV RIM FRAGMENTS MISSING, 3 PROBE HOLES IN BODY/BASE, 1402 INKED ON BASE

SIMPLE BOWL ('HELMUT' SHAPE) WITH FLARED, BEVELLED LIP, ROUNDED BODY AND ROUND BASE

BEVELLED, SL. EVERTED (FLARED)

21.1

2.9

STRAIGHT, OUTSLANTED

SMOOTHED \& BURNISHED (ERODED), PLAIN

$0.5-0.4$

CONVEX

18.5

7.7

SMOOTHED \& BURNISHED (ERODED); ENGRAVED, CONCENTRIC CIRCULAR SPIRAL PATTERN REPEATED $3 \mathrm{X}$

UNDISTINGUISHED/CONVEX

Huddleston Burial 16, 6/5/1938; Phillips photo 3907 (Huddleston 217); Social Hill-Deceiper phase 
Artifact No. / Old Cat. No. / Site No.

Vessel Form

ARAS/HSU Digital Photo

Type

Decoration

Rim :: Body

Paste

Temper

Core Color (Munsell)

Exterior Color (Munsell)

Interior Color (Munsell)

Vessel Overall Height (cm)

Vessel Overall Weight (g)

Vessel Maximum Diameter (cm)

Volume (liters)

Usewear/Sooting/Condition

Shape/Description

Lip Treatment/Shape/Angle/Thickness

Orifice Exterior Diameter $(\mathrm{cm})$

Rim/Neck Height (cm)

Neck Mid/Base Exterior Diameter (cm)

Rim/Neck Shape/Angle

Rim/Neck Surface/Decorative Treatment

Rim/Neck/Body Thickness (cm)

Body Shape

Body Maximum Diameter (cm)

Body Height $(\mathrm{cm})$

Body Surface/Decorative Treatment

Base Shape/Curvature

Base Diameter/Height (cm)

Base Surface/Decorative Treatment

Appendages/Handles

Notes
$5-10 / 1289$ / 3CL81

jar, short rim

3936

incised/punctated

Concord 3 :: Buffalo 14

MEDIUM-HARD, GRANULAR, COMPACT

SHELL (SOME LEACHED), COARSE \& ABUNDANT

VERY DARK GRAY (7.5YR3/1)

V DK GRAY (10YR3/1), GRAY BROWN (10YR5/2), PALE BROWN (10YR6/3)

LIGHT YELLOWISH BROWN (10YR6/4), VERY DARK GRAY (10YR3/1), GRAYISH BROWN (10YR5/2)

12.7

317

13.9

WORN/ERODED/LEACHED SURFACES; BLACKENED RIM INT., SOOT RESIDUE EXT. UPPER BODY, FIRECLOUDING; VESSEL INTACT EXCEPT A FEW SMALL CHIPS AROUND LIP, PROBE HOLE IN UPPER BODY

SHORT-RIM JAR WITH FLARED, FLATTENED LIP, GLOBULAR BODY, AND SLIGHTLY ROUNDED BASE

FLATTENED, EVERTED (FLARED)

13.6

2.0

STRAIGHT, OUTSLANTED

SMOOTHED; PUNCTATED, 3 HORIZONTAL ROWS (OR VERTICAL ROWS OF 3) PUNCTATE LINES AROUND RIM

$0.5-0.4$

GLOBULAR

13.9

10.7

SMOOTHED, INCISED/TRAILED, SPIRAL DESIGN REPEATED 3X AROUND BODY

UNDISTINGUISHED/CONVEX

Huddleston Burial 11, 5/20/1938; Phillips photo 3856 (Huddleston 56); Social Hill phase 
JEC Hodges Collection, 77-1

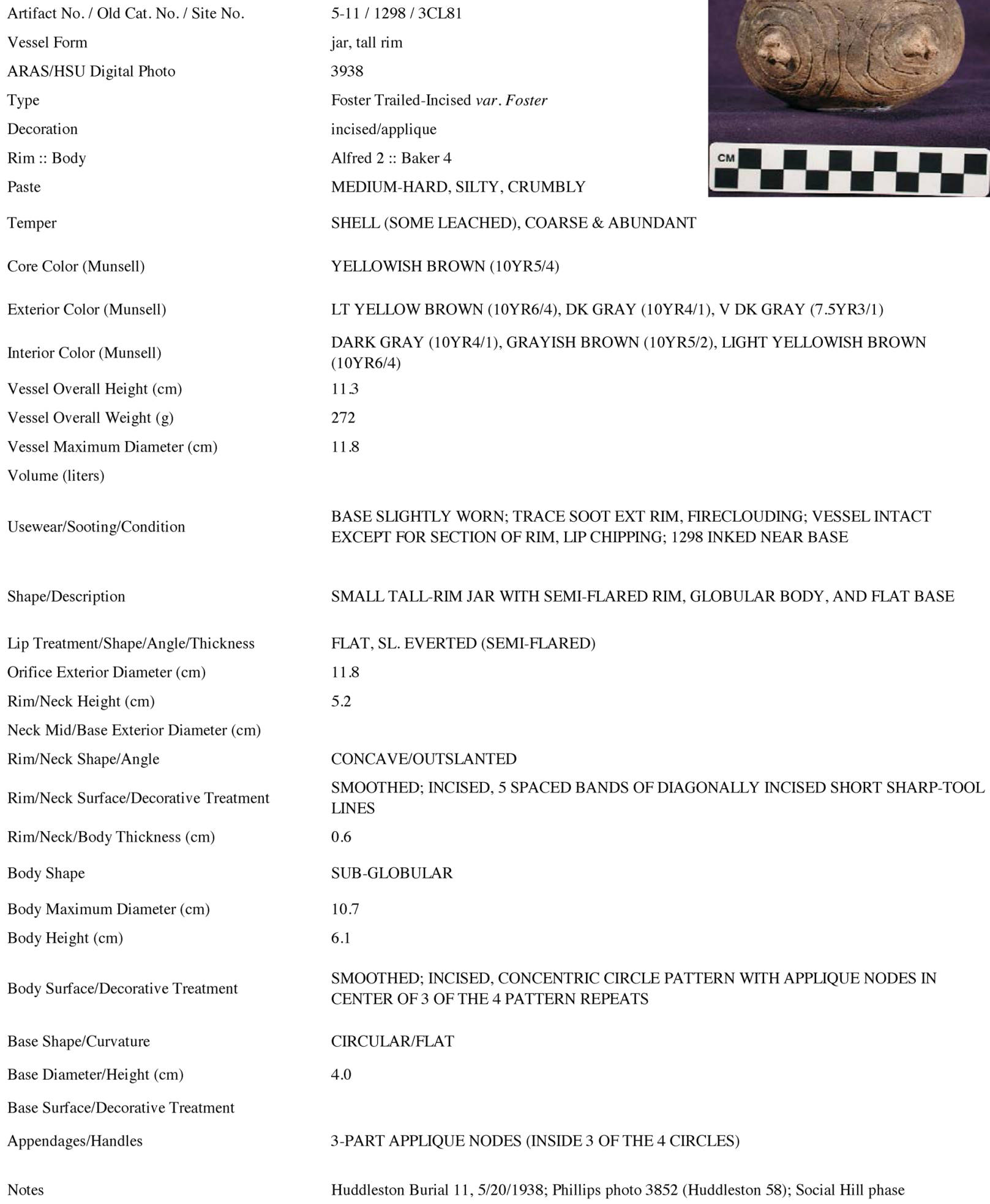

BASE SLIGHTLY WORN; TRACE SOOT EXT RIM, FIRECLOUDING; VESSEL INTACT EXCEPT FOR SECTION OF RIM, LIP CHIPPING; 1298 INKED NEAR BASE

SMALL TALL-RIM JAR WITH SEMI-FLARED RIM, GLOBULAR BODY, AND FLAT BASE

FLAT, SL. EVERTED (SEMI-FLARED)

11.8

5.2

CONCAVE/OUTSLANTED

SMOOTHED; INCISED, 5 SPACED BANDS OF DIAGONALLY INCISED SHORT SHARP-TOOL LINES

0.6

SUB-GLOBULAR

10.7

6.1

SMOOTHED; INCISED, CONCENTRIC CIRCLE PATTERN WITH APPLIQUE NODES IN CENTER OF 3 OF THE 4 PATTERN REPEATS

CIRCULAR/FLAT

4.0

3-PART APPLIQUE NODES (INSIDE 3 OF THE 4 CIRCLES)

Huddleston Burial 11, 5/20/1938; Phillips photo 3852 (Huddleston 58); Social Hill phase 


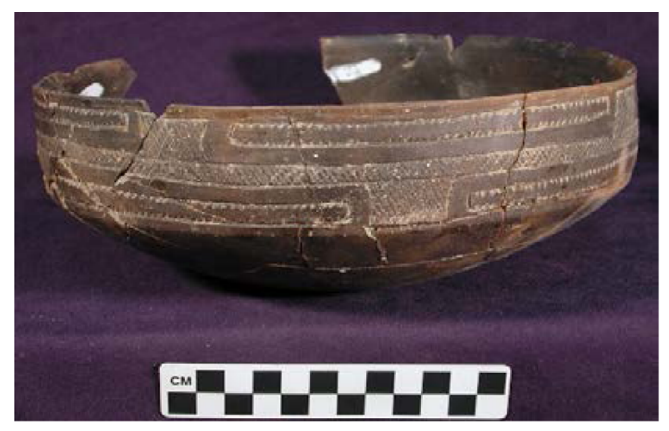

Artifact No. / Old Cat. No. / Site No.

Vessel Form

ARAS/HSU Digital Photo

Type

Decoration

Rim :: Body

Paste

Temper

Core Color (Munsell)

Exterior Color (Munsell)

Interior Color (Munsell)

Vessel Overall Height $(\mathrm{cm})$

Vessel Overall Weight (g)

Vessel Maximum Diameter $(\mathrm{cm})$

Volume (liters)

Usewear/Sooting/Condition

Shape/Description

Lip Treatment/Shape/Angle/Thickness

Orifice Exterior Diameter $(\mathrm{cm})$

Rim/Neck Height (cm)

Neck Mid/Base Exterior Diameter (cm)

Rim/Neck Shape/Angle

Rim/Neck Surface/Decorative Treatment

Rim/Neck/Body Thickness (cm)

Body Shape

Body Maximum Diameter $(\mathrm{cm})$

Body Height $(\mathrm{cm})$

Body Surface/Decorative Treatment

Base Shape/Curvature

Base Diameter/Height $(\mathrm{cm})$

Base Surface/Decorative Treatment

Appendages/Handles

Notes
5-12/1244 / 3CL81

bowl, carinated

3940

Friendship Engraved var. Freeman

engraved

Elaine 3 :: plain

HARD, SILTY, COMPACT

SHELL (SOME LEACHED) \& GROG \& GRIT, FINE

YELLOWISH RED (5YR5/6), GRAY (5YR5/1)

V DK GRAY (7.5YR3/1), DK GRAY (10YR4/1), YELLOWISH RED (5YR5/6)

REDDISH BROWN (5YR5/4), VERY DARK GRAY (7.5YR3/1), DARK GRAY (10YR4/1)

10.0

644

23.2

FIRECLOUDING; VESSEL RECONSTRUCTED WITH GLUE IN PAST, SEVERAL RIM FRAGMENTS MISSING, LIP CHIPPED

LARGE CARINATED BOWL WITH DIRECT RIM, FLARED AND ROUNDED LIP, SHALLOW BODY AND ROUND BASE

ROUNDED, EVERTED (FLARED)

23.0

4.6

CONVEX, STRAIGHT

SMOOTHED \& BURNISHED; ENGRAVED, 3 TIERS WITH OVALS, TICKED LINES ON INTERIOR, CROSSHATCHING BACKGROUND

$0.4-0.5$

CONVEX

23.2

5.4

SMOOTHED \& BURNISHED; PLAIN

UNDISTINGUISHED/CONVEX

Huddleston Burial 12, 5/30-5/31/1938; Phillips photo 3984 (Huddleston 63); Mid-Ouachita phase 
JEC Hodges Collection, 77-1

Artifact No. / Old Cat. No. / Site No.

Vessel Form

ARAS/HSU Digital Photo

Type

Decoration

Rim :: Body

Paste

Temper

Core Color (Munsell)

Exterior Color (Munsell)

Interior Color (Munsell)

Vessel Overall Height $(\mathrm{cm})$

Vessel Overall Weight (g)

Vessel Maximum Diameter (cm)

Volume (liters)

Usewear/Sooting/Condition

Shape/Description

Lip Treatment/Shape/Angle/Thickness

Orifice Exterior Diameter (cm)

Rim/Neck Height (cm)

Neck Mid/Base Exterior Diameter $(\mathrm{cm})$

Rim/Neck Shape/Angle

Rim/Neck Surface/Decorative Treatment

Rim/Neck/Body Thickness (cm)

Body Shape

Body Maximum Diameter $(\mathrm{cm})$

Body Height (cm)

Body Surface/Decorative Treatment

Base Shape/Curvature

Base Diameter/Height (cm)

Base Surface/Decorative Treatment

Appendages/Handles

Notes
$5-13 / 2787$ / 3CL81

jar, tall rim

3942

incised

Albertus 4 :: Abraham 1

HARD, GRANULAR, CRUMBLY

SHELL (SOME LEACHED), COARSE \& ABUNDANT

GRAY (10YR5/1)

DK GRAY (7.5YR4/1), LT YELLOW BRN (10YR6/4), V PALE BROWN (10YR7/3)

DARK GRAY (10YR4/1), LIGHT YELLOWISH BROWN (10YR6/4), REDDISH YELLOW (7.5YR6/6)

11.5

216

12.9

BASE SLIGHTLY WORN; FIRECLOUDING; VESSEL RECONSTRUCTED WITH GLUE, CHIPPED LIP; 2787 INKED NEAR BASE

TALL-RIM JAR WITH A FLARED AND ROUNDED LIP, ROUNDED BODY, AND FLATTENED BASE

ROUNDED; EVERTED (FLARED)

12.9

5.5

CONCAVE, STRAIGHT

SMOOTHED; INCISED, IRREG INCISED DIAGONAL LINES AROUND RIM

0.5

SUB-GLOBULAR

10.7

6.0

SMOOTHED; INCISED, IRREG VERTICAL LINES AROUND UPPER 1/2 OF BODY

CIRCULAR, FLATTENED

4.5

Huddleston Burial X, 2/28/1943; Social Hill-Deceiper phase 
Artifact No. / Old Cat. No. / Site No.

Vessel Form

ARAS/HSU Digital Photo

Type

Decoration

Rim :: Body

Paste

Temper

Core Color (Munsell)

Exterior Color (Munsell)

Interior Color (Munsell)

Vessel Overall Height (cm)

Vessel Overall Weight (g)

Vessel Maximum Diameter (cm)

Volume (liters)

Usewear/Sooting/Condition

Shape/Description

Lip Treatment/Shape/Angle/Thickness

Orifice Exterior Diameter $(\mathrm{cm})$

Rim/Neck Height (cm)

Neck Mid/Base Exterior Diameter (cm)

Rim/Neck Shape/Angle

Rim/Neck Surface/Decorative Treatment

Rim/Neck/Body Thickness (cm)

Body Shape

Body Maximum Diameter (cm)

Body Height $(\mathrm{cm})$

Body Surface/Decorative Treatment

Base Shape/Curvature

Base Diameter/Height (cm)

Base Surface/Decorative Treatment

Appendages/Handles

Notes
5-14 / 1245 / 3CL81

jar, tall rim

3944

Foster Trailed-Incised

incised

Alfred $2::$ Bellarmine 13 (type)

SOFT, GRANULAR, CRUMBLY

SHELL (SOME LEACHED), FINE TO COARSE, ABUNDANT

DARK GRAY (5YR4/1), VERY DARK GRAY (10YR3/1)

YELLOWISH RED (5YR5/6), BLACK (5YR2.5/1), DARK GRAY (10YR4/1)

VERY DARK GRAY (10YR3/1), DARK GRAYISH BROWN (10YR4/2), BROWNISH YELLOW (10YR6/6)

9.5

164

13.8

WORN/ERODED/LEACHED SURFACE; BLACKENED RIM INT. \& BODY EXT., FIRECLOUDING; VESSEL RECONSTRUCTED WITH GLUE IN PAST, SEVERAL FRAGMENTS MISSING/UNATTACHED FROM BODY/RIM

TALL-RIM JAR WITH FLARED AND FLATTENED LIP, FLATTENED GLOBULAR BODY, AND FLATTENED BASE

FLATTENED, EVERTED (FLARED)

13.8

4.0

STRAIGHT, OUTSLANTED

SMOOTHED; INCISED, SHARP-TOOL SHORT INCISED DIAGONAL BANDS (4) ENCIRCLING RIM, WIDE SPACING

$0.3-0.4$

SUB-GLOBULAR

11.4

5.5

SMOOTHED; INCISED, CONCENTRIC HALFCIRCLE PATTERN REPEATED 3X

CIRCULAR, FLATTENED

5.0

Huddleston Burial 17, 6/11/1938; Phillips photo 3862 (Huddleston 64); Social Hill phase 
JEC Hodges Collection, 77-1

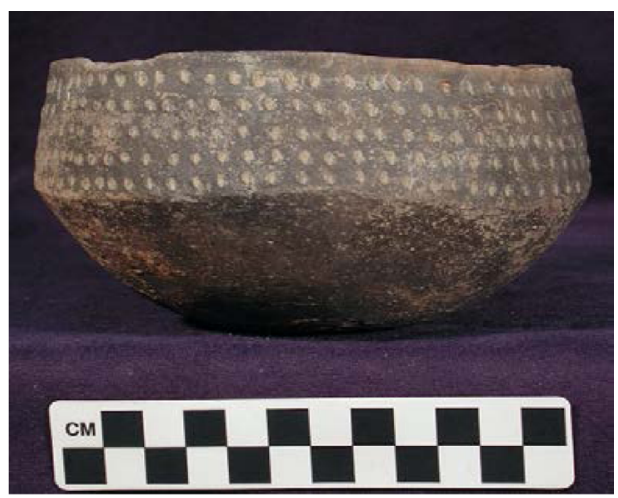

Artifact No. / Old Cat. No. / Site No.

Vessel Form

ARAS/HSU Digital Photo

Type

Decoration

Rim :: Body

Paste

Temper

Core Color (Munsell)

Exterior Color (Munsell)

Interior Color (Munsell)

Vessel Overall Height $(\mathrm{cm})$

Vessel Overall Weight (g)

Vessel Maximum Diameter (cm)

Volume (liters)

Usewear/Sooting/Condition

Shape/Description

Lip Treatment/Shape/Angle/Thickness

Orifice Exterior Diameter $(\mathrm{cm})$

Rim/Neck Height (cm)

Neck Mid/Base Exterior Diameter (cm)

Rim/Neck Shape/Angle

Rim/Neck Surface/Decorative Treatment

Rim/Neck/Body Thickness (cm)

Body Shape

Body Maximum Diameter (cm)

Body Height $(\mathrm{cm})$

Body Surface/Decorative Treatment

Base Shape/Curvature

Base Diameter/Height $(\mathrm{cm})$

Base Surface/Decorative Treatment

Appendages/Handles

Notes
5-15/1308 / 3CL81

bowl, carinated

3946

Sandford Punctated

punctated

Caldwell 3 :: plain

HARD, SILTY, COMPACT

SHELL (LEACHED), FINE \& ABUNDANT

DARK GRAY (10YR4/1)

DARK GRAY (10YR4/1), BLACK (7.5YR2.5/1), YELLOWISH RED (5YR5/6)

VERY DARK GRAY (10YR3/1), DARK GRAY (10YR4/1), GRAYISH BROWN (10YR5/2)

7.6

292

13.7

A FEW MODERATELY WORN SPOTS AROUND VESSEL; FIRECLOUDING; VESSEL RECONSTRUCTED WITH GLUE, CRACK, RIM CHIPPED

SMALL CARINATED BOWL WITH A VERY SLIGHTLY FLARED AND ROUNDED LIP, ROUNDED BODY AND BASE

ROUNDED, SL. EVERTED (SL. FLARED)

12.5

3.5

STRAIGHT, INSLANTED/CARINATED

SMOOTHED; PUNCTATED, 5 HORIZONTAL ROWS OF CIRCULAR TOOL PUNCTATES AROUND RIM

$0.4-0.5$

CONVEX

13.7

4.1

SMOOTHED; PLAIN

UNDISTINGUISHED, CONVEX

Huddleston Burial 11, 5/20/1938; Phillips photo 3878 (Huddleston 55); Social Hill phase 
JEC Hodges Collection, 77-1

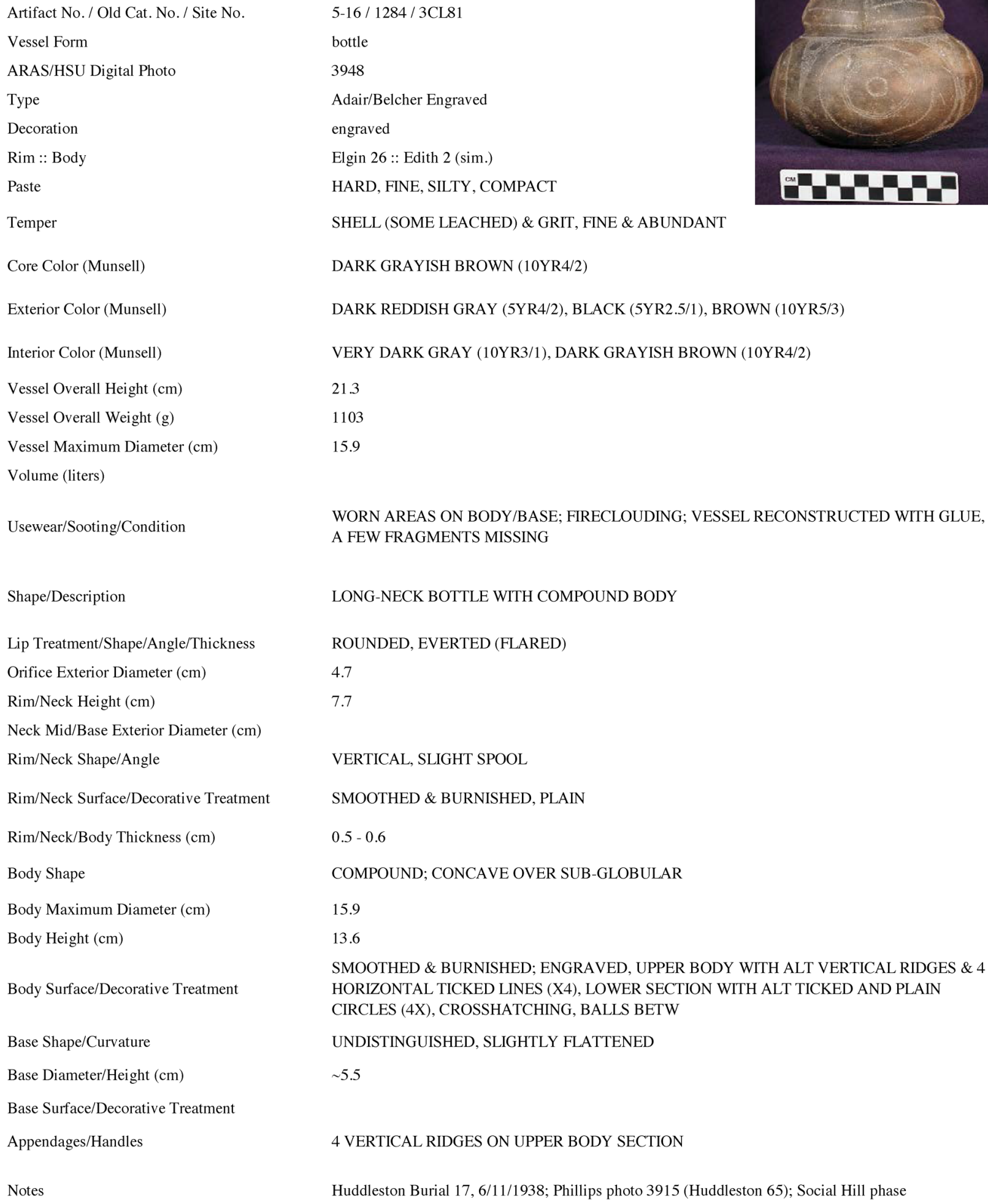

WORN AREAS ON BODY/BASE; FIRECLOUDING; VESSEL RECONSTRUCTED WITH GLUE, A FEW FRAGMENTS MISSING

LONG-NECK BOTTLE WITH COMPOUND BODY

ROUNDED, EVERTED (FLARED)

4.7

7.7

VERTICAL, SLIGHT SPOOL

SMOOTHED \& BURNISHED, PLAIN

$0.5-0.6$

COMPOUND; CONCAVE OVER SUB-GLOBULAR

15.9

13.6

SMOOTHED \& BURNISHED; ENGRAVED, UPPER BODY WITH ALT VERTICAL RIDGES \& 4 HORIZONTAL TICKED LINES (X4), LOWER SECTION WITH ALT TICKED AND PLAIN CIRCLES (4X), CROSSHATCHING, BALLS BETW

UNDISTINGUISHED, SLIGHTLY FLATTENED

$\sim 5.5$

4 VERTICAL RIDGES ON UPPER BODY SECTION

Huddleston Burial 17, 6/11/1938; Phillips photo 3915 (Huddleston 65); Social Hill phase 
JEC Hodges Collection, 77-1

Artifact No. / Old Cat. No. / Site No.

Vessel Form

ARAS/HSU Digital Photo

Type

Decoration

$\operatorname{Rim}::$ Body

Paste

Temper

Core Color (Munsell)

Exterior Color (Munsell)

Interior Color (Munsell)

Vessel Overall Height $(\mathrm{cm})$

Vessel Overall Weight (g)

Vessel Maximum Diameter (cm)

Volume (liters)

Usewear/Sooting/Condition

Shape/Description

Lip Treatment/Shape/Angle/Thickness

Orifice Exterior Diameter $(\mathrm{cm})$

Rim/Neck Height (cm)

Neck Mid/Base Exterior Diameter (cm)

Rim/Neck Shape/Angle

Rim/Neck Surface/Decorative Treatment

Rim/Neck/Body Thickness (cm)

Body Shape

Body Maximum Diameter (cm)

Body Height $(\mathrm{cm})$

Body Surface/Decorative Treatment

Base Shape/Curvature

Base Diameter/Height $(\mathrm{cm})$

Base Surface/Decorative Treatment

Appendages/Handles

Notes
5-17/1436 / 3CL81

bowl, carinated

3952

Friendship Engr. var. Trigg?

engraved

Erie $2::$ Emporia 2 :: plain

HARD, SILTY, COMPACT

SHELL (SOME LEACHED), FINE \& ABUNDANT

YELLOW RED (5YR5/6), GRAYISH BROWN (10YR5/2), V DK GRAY (10YR3/1)

YELLOWISH BROWN (10YR5/4), DARK GRAY (10YR4/1), VERY DARK GRAY (10YR3/1)

10.0

553

19.7

BASE SLIGHTLY WORN; FIRECLOUDING; VESSEL INTACT; 1436 INKED ON BASE

LARGE CARINATED BOWL WITH FLARED AND ROUNDED LIP, SHALLOW ROUNDED BODY, AND ROUND BASE

ROUNDED, EVERTED (FLARED)

19.7

5.7

CONVEX, VERTICAL

SMOOTHED \& BURNISHED; ENGRAVED, POSITIVE/NEGATIVE DESIGN OF SCROLLS, CROSSHATCHED BACKGROUND; WHITE PIGMENT IN ENGRAVING

0.5

CONVEX

19.3

4.3

SMOOTHED \& BURNISHED; PLAIN

UNDISTINGUISHED, CONVEX

Huddleston Burial 8, 5/28/1938; Phillips photo 3903 (Huddleston 52); Mid-Ouachita phase? 
JEC Hodges Collection, 77-1

Artifact No. / Old Cat. No. / Site No.

Vessel Form

ARAS/HSU Digital Photo

Type

Decoration

Rim :: Body

Paste

Temper

Core Color (Munsell)

Exterior Color (Munsell)

Interior Color (Munsell)

Vessel Overall Height $(\mathrm{cm})$

Vessel Overall Weight (g)

Vessel Maximum Diameter $(\mathrm{cm})$

Volume (liters)

Usewear/Sooting/Condition

Shape/Description

Lip Treatment/Shape/Angle/Thickness Orifice Exterior Diameter $(\mathrm{cm})$

Rim/Neck Height (cm)

Neck Mid/Base Exterior Diameter (cm)

Rim/Neck Shape/Angle

Rim/Neck Surface/Decorative Treatment

Rim/Neck/Body Thickness (cm)

Body Shape

Body Maximum Diameter (cm)

Body Height $(\mathrm{cm})$

Body Surface/Decorative Treatment

Base Shape/Curvature

Base Diameter/Height $(\mathrm{cm})$

Base Surface/Decorative Treatment

Appendages/Handles

Notes
5-18/1238/3CL81

bottle

3954

plain

MEDIUM, SILTY, SLIGHTLY CRUMBLY

SHELL (LEACHED), FINE, MODERATELY ABUNDANT

GRAY (10YR5/1)

LT BROWNISH GRAY (10YR6/2), V PALE BROWN (10YR7/3), GRAY (10YR5/1)

GRAY (10YR5/1)

29.9

896

21.0

BASE MODERATELY TO HEAVILY WORN; FIRECLOUDING; VESSEL RECONSTRUCTED WITH GLUE IN PAST, ONE PC MISSING FROM BODY, CHIPPED LIP; 1238 INKED NEAR BASE

LARGE LONG-NECK BOTTLE WITH A FLARED AND ROUNDED LIP, VERY SLIGHTLY SQUAT-GLOBULAR BODY, AND FLATTENED BASE

ROUNDED, SL. EVERTED

4.5

10.9

VERTICAL, SLIGHT SPOOL

SMOOTHED; PLAIN

0.4

SUB-GLOBULAR

21.0

19.0

SMOOTHED; PLAIN

CIRCULAR, FLATTENED

7.7

Huddleston Burial 3, 5/20/1938; Phillips photo 3986 (Huddleston 46); Mid-Ouachita phase 


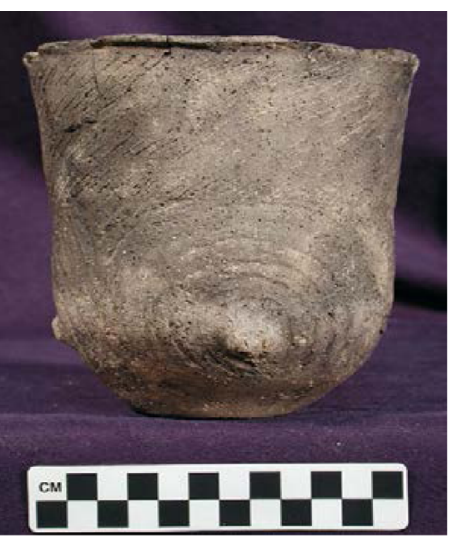

Artifact No. / Old Cat. No. / Site No.

Vessel Form

ARAS/HSU Digital Photo

Type

Decoration

Rim :: Body

Paste

Temper

Core Color (Munsell)

Exterior Color (Munsell)

Interior Color (Munsell)

Vessel Overall Height $(\mathrm{cm})$

Vessel Overall Weight (g)

Vessel Maximum Diameter (cm)

Volume (liters)

Usewear/Sooting/Condition

Shape/Description

Lip Treatment/Shape/Angle/Thickness Orifice Exterior Diameter (cm)

Rim/Neck Height (cm)

Neck Mid/Base Exterior Diameter $(\mathrm{cm})$

Rim/Neck Shape/Angle

Rim/Neck Surface/Decorative Treatment

Rim/Neck/Body Thickness (cm)

Body Shape

Body Maximum Diameter (cm)

Body Height $(\mathrm{cm})$

Body Surface/Decorative Treatment

Base Shape/Curvature

Base Diameter/Height $(\mathrm{cm})$

Base Surface/Decorative Treatment

Appendages/Handles

Notes
5-19/1240 / 3CL81

jar, tall rim

3956

Foster Trailed-Incised

incised/applique

Agnes 1 :: Baker 26 (sim.)

HARD, GRANULAR, CRUMBLY

SHELL (SOME LEACHED), COARSE \& ABUNDANT

BLACK (10YR2/1), DARK GRAY (10YR4/1)

V DK GRAY (10YR3/1), DK GRAY (10YR4/1), LT YELLOWISH BROWN (10YR6/4)

BLACK (10YR2/1), VERY DARK GRAY (10YR3/1)

13.8

375

13.7

SLIGHTLY TO MODERATELY WORN/ERODED/CRUMBLY; FIRECLOUDING; VESSEL RECONSTRUCTED WITH GLUE IN PAST, SEVERAL MISSING PCS, PROBE HOLE LOWER BODY

TALL-RIM JAR WITH FLARED, SLIGHTLY FLATTENED LIP, SLIGHTLY ROUNDED BASE, AND FLAT BASE

SLIGHTLY FLATTENED, EVERTED (FLARED)

13.7

6.2

STRAIGHT, SL. OUTSLANTED

SMOOTHED; INCISED, 4 HORIZONTAL ROWS OF DIAGONALLY INCISED SHORT LINES

0.5

SUB-GLOBULAR

11.6

7.6

SMOOTHED; INCISED BROAD CONCENTRIC CIRCLES (X3), 2-PRONG APPLIQUE NODES (ONE MISSING) IN THE CENTER OF EACH; DESIGN ON UPPER 3/4 BODY

CIRCULAR, FLAT

5.6

2-PRONG APPLIQUE NODES ON BODY (ORIG 3, 1 MISSING)

Huddleston Burial 19, 7/18/1938; Phillips photo 3864 (Huddleston 72); Social Hill-Deceiper phase 
Artifact No. / Old Cat. No. / Site No.

Vessel Form

ARAS/HSU Digital Photo

Type

Decoration

Rim :: Body

Paste

Temper

Core Color (Munsell)

Exterior Color (Munsell)

Interior Color (Munsell)

Vessel Overall Height $(\mathrm{cm})$

Vessel Overall Weight (g)

Vessel Maximum Diameter $(\mathrm{cm})$

Volume (liters)

Usewear/Sooting/Condition

Shape/Description

Lip Treatment/Shape/Angle/Thickness Orifice Exterior Diameter $(\mathrm{cm})$

Rim/Neck Height $(\mathrm{cm})$

Neck Mid/Base Exterior Diameter (cm)

Rim/Neck Shape/Angle

Rim/Neck Surface/Decorative Treatment

Rim/Neck/Body Thickness (cm)

Body Shape

Body Maximum Diameter $(\mathrm{cm})$

Body Height $(\mathrm{cm})$

Body Surface/Decorative Treatment

Base Shape/Curvature

Base Diameter/Height $(\mathrm{cm})$

Base Surface/Decorative Treatment

Appendages/Handles

Notes
5-20 / $1290 / 3$ CL81

seed jar

3958

plain

MEDIUM-HARD, SILTY, COMPACT

SHELL (PARTLY LEACHED), FINE \& ABUNDANT

LT YELLOWISH BROWN (10YR6/4), GRAY (10YR6/1), DK GRAY (10YR4/1)

16.1

1461

20.1

BASE MODERATELY WORN; FIRECLOUDING; VESSEL INTACT; 1290 INKED ON LOWER BODY

LARGE PLAIN SEED JAR WITH A LOW-WAISTED BODY AND FLAT BASE

FLATTENED/BEVELED IN

4.0

CONVEX, INSLANTED, CONTINUOUS WITH BODY

SMOOTHED; PLAIN

0.8

LOW-WAISTED

20.1

16.1

SMOOTHED; PLAIN

CIRCULAR, FLAT

12.0

Huddleston Burial 12, 5/30-5/31/1938; Phillips photo 3905 (Huddleston 59); Mid-Ouachita phase 


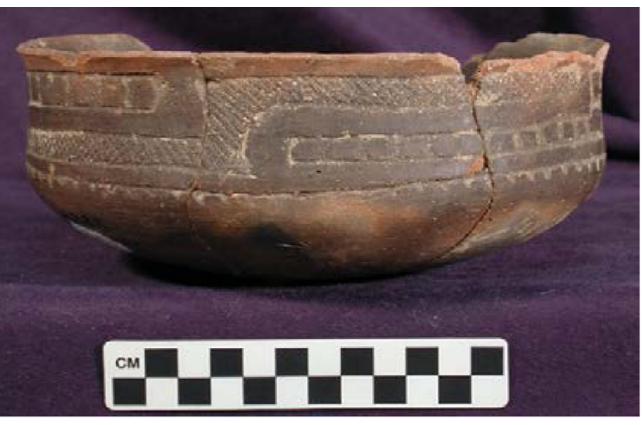

Artifact No. / Old Cat. No. / Site No.

Vessel Form

ARAS/HSU Digital Photo

Type

Decoration

Rim :: Body

Paste

Temper

Core Color (Munsell)

Exterior Color (Munsell)

Interior Color (Munsell)

Vessel Overall Height $(\mathrm{cm})$

Vessel Overall Weight (g)

Vessel Maximum Diameter $(\mathrm{cm})$

Volume (liters)

Usewear/Sooting/Condition

Shape/Description

Lip Treatment/Shape/Angle/Thickness Orifice Exterior Diameter (cm)

Rim/Neck Height (cm)

Neck Mid/Base Exterior Diameter (cm)

Rim/Neck Shape/Angle

Rim/Neck Surface/Decorative Treatment

Rim/Neck/Body Thickness (cm)

Body Shape

Body Maximum Diameter $(\mathrm{cm})$

Body Height $(\mathrm{cm})$

Body Surface/Decorative Treatment

Base Shape/Curvature

Base Diameter/Height (cm)

Base Surface/Decorative Treatment

Appendages/Handles

Notes
5-21/ 1283 / 3CL81

bowl, carinated

3960

Friendship Engraved var. Meador

engraved

Elwyn 2 :: plain

HARD, SILTY, COMPACT

GROG, FINE \& ABUNDANT, SOME GRIT?

REDDISH BROWN (5YR5/4)

REDDISH BROWN (5YR5/4), VERY DARK GRAY (10YR3/1), BLACK (10YR2/1)

REDDISH BROWN (5YR5/4), VERY DARK GRAY (10YR3/1), DARK GRAY (10YR4/1)

8.8

428

19.4

BASE IS MOD TO HEAVILY WORN; FIRECLOUDING; VESSEL RECONSTRUCTED WITH GLUE IN PAST, LARGE RIM PORTION MISSING, LIP CHIPPED; 1283 INKED NEAR BASE

CARINATED BOWL WITH FLARED AND ROUNDED LIP, AND A ROUNDED BODY/BASE

ROUNDED, EVERTED (FLARED)

19.4

4.5

CONCAVE, VERTICAL

SMOOTHED \& LIGHTLY BURNISHED; ENGRAVED, 2 OVAL PANELS LINKED BY LSHAPED CROSSHATCHING, LADDERS, TICKED LINE AT CARINATION

$0.5-0.4$

CONVEX

19.0

4.3

SMOOTHED \& BURNISHED (LIGHTLY); PLAIN

UNDISTINGUISHED, CONVEX

Huddleston Burial 23, 11/26/1938; Phillips photo 3952 (Huddleston 88); early Mid-Ouachita phase 


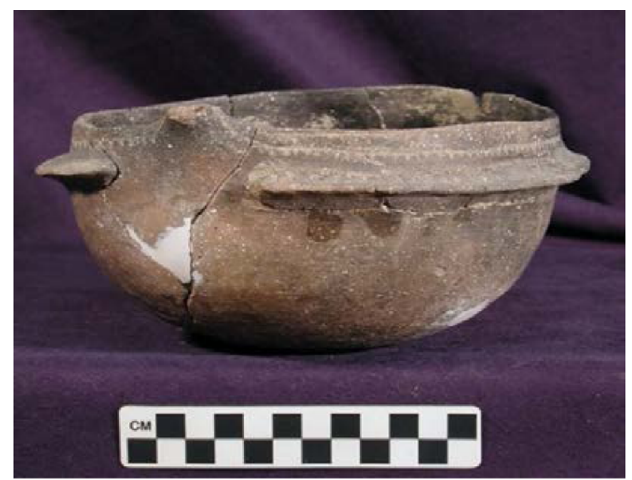

Artifact No. / Old Cat. No. / Site No.

Vessel Form

ARAS/HSU Digital Photo

Type

Decoration

Rim :: Body

Paste

Temper

Core Color (Munsell)

Exterior Color (Munsell)

Interior Color (Munsell)

Vessel Overall Height $(\mathrm{cm})$

Vessel Overall Weight (g)

Vessel Maximum Diameter (cm)

Volume (liters)

Usewear/Sooting/Condition

Shape/Description

Lip Treatment/Shape/Angle/Thickness

Orifice Exterior Diameter $(\mathrm{cm})$

Rim/Neck Height (cm)

Neck Mid/Base Exterior Diameter $(\mathrm{cm})$

Rim/Neck Shape/Angle

Rim/Neck Surface/Decorative Treatment

Rim/Neck/Body Thickness (cm)

Body Shape

Body Maximum Diameter (cm)

Body Height $(\mathrm{cm})$

Body Surface/Decorative Treatment

Base Shape/Curvature

Base Diameter/Height $(\mathrm{cm})$

Base Surface/Decorative Treatment

Appendages/Handles

Notes
5-23 / 2399 / 3CL81

effigy bowl

3962

Means Engraved

engraved/appliqued flange/handles

Elgin $1::$ plain

HARD, SILTY, COMPACT

SHELL (SOME LEACHED), FINE/ABUNDANT

VERY DARK GRAY (10YR3/1), DARK GRAY (10YR4/1)

LT YELLOW BROWN (10YR6/4), DK GRAY BROWN (10YR4/2), BLACK (10YR2/1)

BLACK (10YR2/1), VERY DARK GRAY (10YR3/1), DARK GRAY (10YR4/1)

10.7

428

18.8

SLIGHT TO MODERATE WEAR ALL AROUND; VESSEL RECONSTRUCTED WITH GLUE, EFFIGY HEAD BROKEN OFF

SIMPLE BOWL WITH EFFIGY HEAD BROKEN OFF AND FLANGE AROUND RIM (BIRD?)

\section{FLATTENED, NONE (DIRECT)}

16.1

3.0

CONVEX, VERTICAL, CONTINUOUS WITH BODY

ENGRAVED WITH APPLIQUE, SINGLE TICKED LINE BELOW LIP; APPLIQUE FLANGE BELOW TICKED LINE (BIRD WINGS WITH PRONGED HEAD/TAIL?)

0.4

CONVEX, ROUNDED

18.8

7.7

SMOOTHED \& BURNISHED (LIGHTLY); PLAIN

UNDISTINGUISHED, CONVEX

\section{APPLIQUE FLANGE BELOW LIP, EFFIGY HEAD BROKEN OFF}

Huddleston Burial 26 (27?), 1/4/1940 or 1/4/1941 (described by Huddleston as turtle effigy), MidOuachita - Social Hill phase; publ. Trubitt 2017 


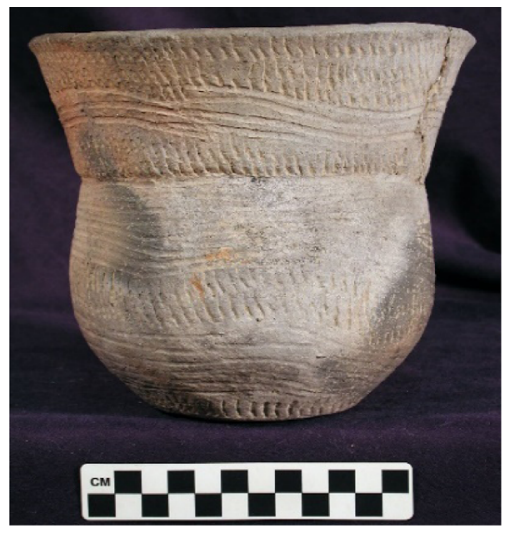

Artifact No. / Old Cat. No. / Site No.

Vessel Form

ARAS/HSU Digital Photo

Type

Decoration

Rim :: Body

Paste

Temper

Core Color (Munsell)

Exterior Color (Munsell)

Interior Color (Munsell)

Vessel Overall Height $(\mathrm{cm})$

Vessel Overall Weight (g)

Vessel Maximum Diameter $(\mathrm{cm})$

Volume (liters)

Usewear/Sooting/Condition

Shape/Description

Lip Treatment/Shape/Angle/Thickness

Orifice Exterior Diameter $(\mathrm{cm})$

Rim/Neck Height (cm)

Neck Mid/Base Exterior Diameter $(\mathrm{cm})$

Rim/Neck Shape/Angle

Rim/Neck Surface/Decorative Treatment

Rim/Neck/Body Thickness (cm)

Body Shape

Body Maximum Diameter (cm)

Body Height $(\mathrm{cm})$

Body Surface/Decorative Treatment

Base Shape/Curvature

Base Diameter/Height (cm)

Base Surface/Decorative Treatment

Appendages/Handles

Notes
5-24 / 1285 / 3CL81

jar, tall rim

3964

Military Road Incised

incised + punctated

Bethel 1 :: Bard 2

HARD, SILTY, COMPACT

GROG, FINE \& ABUNDANT + SHELL, SPARSE

PALE BROWN (10YR6/3), VERY PALE BROWN (10YR7/3), DARK GRAY (7.5YR4/1)

DARK GRAY (7.5YR4/1), PALE BROWN (10YR6/3), YELLOWISH RED (5YR5/6)

GRAY (10YR6/1), REDDISH YELLOW (7.5YR6/6), VERY DARK GRAY (10YR3/1)

16.5

819

17.2

FIRECLOUDING, BLACKENED INTERIOR; RIM RECONSTR W GLUE IN PAST, SMALL CRACKS ON BODY; 1285 INKED ON INT RIM

TALL-RIM JAR WITH FLARED, FLATTENED LIP, SUBGLOBULAR BODY, AND FLAT BASE

FLATTENED, EVERTED (FLARED)

17.2

6.0

\section{STRAIGHT, OUTSLANTED}

SMOOTHED; INCISED/TRAILED + PUNCTATED, MEANDERING LINES WITH VERTICAL PUNCTATES ABOVE AND BELOW

0.6

SUBGLOBULAR

14.3

10.5

SMOOTHED; INCISED/TRAILED MEANDERING LINES ALTERNATING WITH ROWS OF VERTICAL PUNCTATES

CIRCULAR, FLAT

8.0

Huddleston Burial 21, 11/26/1938; Phillips photo 3912 (Huddleston 90); Mid-Ouachita phase 


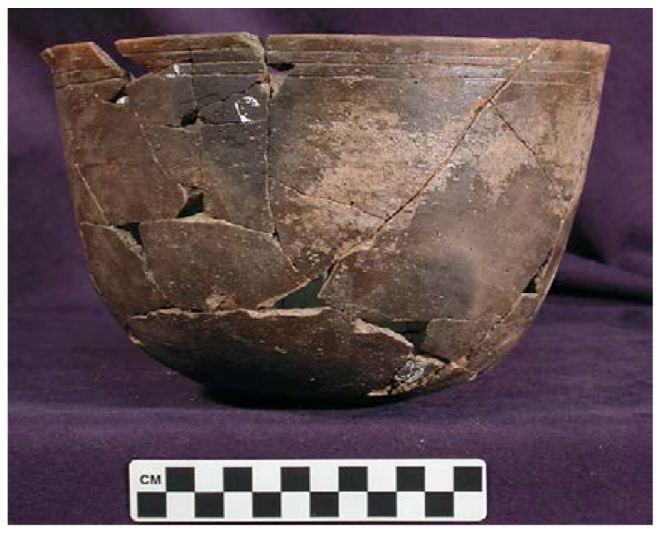

Artifact No. / Old Cat. No. / Site No.

Vessel Form

ARAS/HSU Digital Photo

Type

Decoration

Rim :: Body

Paste

Temper

Core Color (Munsell)

Exterior Color (Munsell)

Interior Color (Munsell)

Vessel Overall Height $(\mathrm{cm})$

Vessel Overall Weight (g)

Vessel Maximum Diameter (cm)

Volume (liters)

Usewear/Sooting/Condition

Shape/Description

Lip Treatment/Shape/Angle/Thickness

Orifice Exterior Diameter $(\mathrm{cm})$

Rim/Neck Height (cm)

Neck Mid/Base Exterior Diameter (cm)

Rim/Neck Shape/Angle

Rim/Neck Surface/Decorative Treatment

Rim/Neck/Body Thickness (cm)

Body Shape

Body Maximum Diameter (cm)

Body Height $(\mathrm{cm})$

Body Surface/Decorative Treatment

Base Shape/Curvature

Base Diameter/Height $(\mathrm{cm})$

Base Surface/Decorative Treatment

Appendages/Handles

Notes
5-25 / 3CL81

bowl, simple

3966

Hardman Engraved (similar)

incised

Erie 3 ? Bates 2 ?:: plain

HARD, SILTY, COMPACT

SHELL (SOME LEACHED), FINE \& ABUNDANT

GRAYISH BROWN (10YR5/2)

V DK GRAY (10YR3/1), BLACK (10YR2/1), YELLOW BROWN (10YR5/4)

DARK GRAY (5YR4/1), VERY DARK GRAY (10YR3/1), YELLOWISH BROWN (10YR5/4)

15.0

655

20.7

FIRECLOUDING; SPOTS OF MODERATE TO HEAVY EROSION ON VESSEL; SHERD LOT, RECONSTRUCTED WITH GLUE IN PAST, SEVERAL SHERDS MISSING, NO INKED \#

SIMPLE BOWL WITH SLIGHTLY FLARED AND ROUNDED LIP, U-SHAPED BODY, AND ROUNDED BASE

ROUNDED, SLIGHTLY EVERTED (SLIGHTLY FLARED)

20.7

1.6

CONCAVE, OUTSLANTED

SMOOTHED \& INCISED, 3 FINE HORIZONTAL LINES BELOW LIP

$0.4-0.5$

CONVEX, CONTINUOUS RIM/BODY/BASE

20.0

13.4

SMOOTHED \& BURNISHED (LIGHTLY); PLAIN

UNDISTINGUISHED, CONVEX

Huddleston Burial 11, 5/20/1938; no inked number, 1977 catalog lists as B11 but unclear why; Social Hill phase 


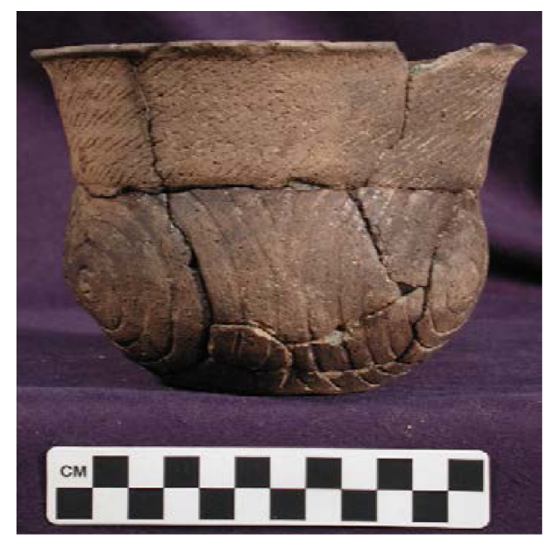

Artifact No. / Old Cat. No. / Site No.

Vessel Form

ARAS/HSU Digital Photo

Type

Decoration

Rim :: Body

Paste

Temper

Core Color (Munsell)

Exterior Color (Munsell)

Interior Color (Munsell)

Vessel Overall Height $(\mathrm{cm})$

Vessel Overall Weight (g)

Vessel Maximum Diameter (cm)

Volume (liters)

Usewear/Sooting/Condition

Shape/Description

Lip Treatment/Shape/Angle/Thickness Orifice Exterior Diameter $(\mathrm{cm})$

Rim/Neck Height (cm)

Neck Mid/Base Exterior Diameter (cm)

Rim/Neck Shape/Angle

Rim/Neck Surface/Decorative Treatment

Rim/Neck/Body Thickness (cm)

Body Shape

Body Maximum Diameter (cm)

Body Height $(\mathrm{cm})$

Body Surface/Decorative Treatment

Base Shape/Curvature

Base Diameter/Height (cm)

Base Surface/Decorative Treatment

Appendages/Handles

Notes
5-28 / 2785 / 3CL81

jar, tall rim

3968

Foster Trailed-Incised

incised

Agnes 1 :: Baker 1

HARD, GRANULAR, COMPACT

SHELL (SOME LEACHED), COARSE \& ABUNDANT

BLACK (10YR2/1), GRAY (10YR5/1)

BROWN (10YR5/3), VERY DARK GRAY (10YR3/1), BLACK (10YR2/1)

BLACK (10YR2/1), VERY DARK GRAY (10YR3/1), DARK GRAYISH BROWN (10YR4/2)

11.2

291

14.3

WEAR/EROSION/LEACHING; FIRECLOUDING, BLACKENED INTERIOR; SHERD LOT, RECONSTRUCTED WITH GLUE IN PAST, SEVERAL SHERDS MISSING

TALL-RIM JAR WITH FLARED AND ROUNDED LIP, SUBGLOBULAR BODY, AND A FLAT BASE

ROUNDED, EVERTED (FLARED)

14.3

4.4

CONCAVE, OUTSLANTED

SMOOTHED; INCISED, 5 ROWS OF DIAGONALLY INCISED LINES

$0.6-0.5$

SUBGLOBULAR

12.6

6.8

SMOOTHED; INCISED/TRAILED, CONCENTRIC CIRCLES REPEATED 3X AROUND BODY, NO CENTRAL NODES

CIRCULAR, FLAT

6.3

Huddleston Burial X, 2/28/1943; Social Hill-Deceiper phase 
JEC Hodges Collection, 77-1

Artifact No. / Old Cat. No. / Site No.

Vessel Form

ARAS/HSU Digital Photo

Type

Decoration

Rim :: Body

Paste

Temper

Core Color (Munsell)

Exterior Color (Munsell)

Interior Color (Munsell)

Vessel Overall Height $(\mathrm{cm})$

Vessel Overall Weight (g)

Vessel Maximum Diameter $(\mathrm{cm})$

Volume (liters)

Usewear/Sooting/Condition

Shape/Description

Lip Treatment/Shape/Angle/Thickness Orifice Exterior Diameter $(\mathrm{cm})$

Rim/Neck Height ( $\mathrm{cm}$ )

Neck Mid/Base Exterior Diameter $(\mathrm{cm})$

Rim/Neck Shape/Angle

Rim/Neck Surface/Decorative Treatment

Rim/Neck/Body Thickness (cm)

Body Shape

Body Maximum Diameter (cm)

Body Height ( $\mathrm{cm}$ )

Body Surface/Decorative Treatment

Base Shape/Curvature

Base Diameter/Height (cm)

Base Surface/Decorative Treatment

Appendages/Handles

Notes
$5-29 / 2410 / 3$ CL81

bowl, simple

3920

engraved

Erie? :: Elmira? (sim.)

MEDIUM-HARD, SILTY, COMPACT

FINE GROG \& GRIT

DARK GRAY (10YR4/1)

BROWN (10YR5/3), LT YELLOW BROWN (10YR6/4), DK GRAY (10YR4/1)

DARK GRAY (10YR4/1), VERY DARK GRAY (7.5YR3/1), GRAYISH BROWN (10YR5/2)

11.3

307

13.7

BASE SLIGHTLY TO MODERATELY WORN; FIRECLOUDING; VESSEL RIM RECONSTRUCTED, LIP MISSING, PROBE HOLE IN BODY

JAR-LIKE VESSEL WITH FLARED RIM, ROUNDED BODY, AND A VERY SLIGHTLY ROUNDED BASE

MISSING

12.6

4.7

STRAIGHT, OUTSLANTED

SMOOTHED; ENGRAVED, 6 HORIZONTAL ENGRAVED BANDS ENCIRCLING RIM

0.5

SUBGLOBULAR

13.7

6.6

SMOOTHED; ENGRAVED, ZONE OF HOOK ELEMENTS EXTENDING UPWARDS FROM BOTTOM LINE WITH CROSSHATCHING INSIDE HOOKS

UNDISTINGUISHED, CONVEX 


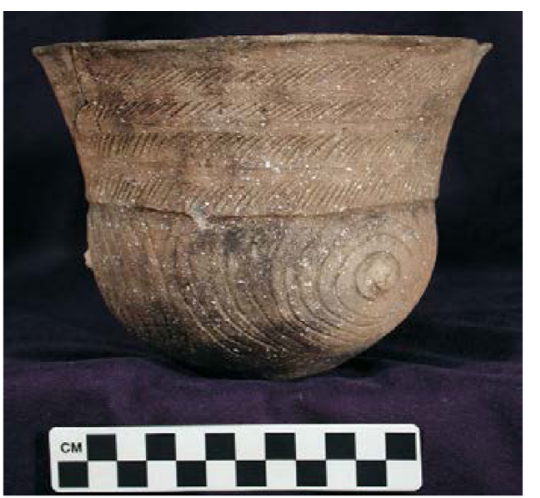

Artifact No. / Old Cat. No. / Site No.

Vessel Form

ARAS/HSU Digital Photo

Type

Decoration

Rim :: Body

Paste

Temper

Core Color (Munsell)

Exterior Color (Munsell)

Interior Color (Munsell)

Vessel Overall Height $(\mathrm{cm})$

Vessel Overall Weight (g)

Vessel Maximum Diameter (cm)

Volume (liters)

Usewear/Sooting/Condition

Shape/Description

Lip Treatment/Shape/Angle/Thickness Orifice Exterior Diameter $(\mathrm{cm})$

Rim/Neck Height (cm)

Neck Mid/Base Exterior Diameter (cm)

Rim/Neck Shape/Angle

Rim/Neck Surface/Decorative Treatment

Rim/Neck/Body Thickness (cm)

Body Shape

Body Maximum Diameter (cm)

Body Height $(\mathrm{cm})$

Body Surface/Decorative Treatment

Base Shape/Curvature

Base Diameter/Height (cm)

Base Surface/Decorative Treatment

Appendages/Handles

Notes
5-30 / 1292 / 3CL81

jar, tall rim

3950

Foster Trailed-Incised var. Foster

incised/applique

Alfred 6 :: Baker 22

MEDIUM, SILTY, SLIGHTLY CRUMBLY

SHELL, COARSE \& ABUNDANT

VERY DARK GRAY (10YR3/1)

V DK GRAY (10YR3/1), BROWN (10YR5/3), LT YELLOW BROWN (10YR6/4)

BROWN (10YR5/3), GRAY (10YR5/1), LIGHT YELLOWISH BROWN (10YR6/4)

13.4

390

16.0

FIRECLOUDING; VESSEL RECONSTRUCTED WITH GLUE, SEVERAL FRAGMENTS OF RIM MISSING, SEVERAL CRACKS AROUND RIM/BODY; 1292 INKED NEAR BASE

TALL-RIM JAR WITH FLARED, ROUNDED LIP, SUBGLOBULAR BODY, AND FLATTENED BASE

ROUNDED, EVERTED (FLARED)

16.0

6.2

CONCAVE, OUTSLANTED

INCISED, 4 SPACED ROWS OF DIAGONAL SHORT TOOL INCISED LINES

$0.6-0.3$

SUBGLOBULAR

12.0

7.2

SMOOTHED; INCISED/TRAILED, CONCENTRIC CIRCLES WITH CENTRAL APPLIQUE SINGLE-PRONG NODE (REPEATED 3X)

CIRCULAR, FLATTENED

5.2

3 SINGLE-PRONG APPLIQUE NODES

Huddleston Burial 11, 5/20/1938; Phillips photo 3911 (Huddleston 54); Social Hill phase 
JEC Hodges Collection, 77-1

Artifact No. / Old Cat. No. / Site No.

Vessel Form

ARAS/HSU Digital Photo

Type

Decoration

$\operatorname{Rim}::$ Body

Paste

Temper

Core Color (Munsell)

Exterior Color (Munsell)

Interior Color (Munsell)

Vessel Overall Height $(\mathrm{cm})$

Vessel Overall Weight (g)

Vessel Maximum Diameter (cm)

Volume (liters)

Usewear/Sooting/Condition

Shape/Description

Lip Treatment/Shape/Angle/Thickness Orifice Exterior Diameter $(\mathrm{cm})$

Rim/Neck Height $(\mathrm{cm})$

Neck Mid/Base Exterior Diameter $(\mathrm{cm})$

Rim/Neck Shape/Angle

Rim/Neck Surface/Decorative Treatment

Rim/Neck/Body Thickness (cm)

Body Shape

Body Maximum Diameter $(\mathrm{cm})$

Body Height $(\mathrm{cm})$

Body Surface/Decorative Treatment

Base Shape/Curvature

Base Diameter/Height $(\mathrm{cm})$

Base Surface/Decorative Treatment

Appendages/Handles
5-31/1307 / 3CL81

bottle

670,686

Keno Trailed var. Red Hill

incised

Bates 5 :: Belhaven 5 (sim.)

HARD, COMPACT

SHELL (SOME LEACHED), MEDIUM \& ABUNDANT

GRAY (10YR5/1)

LT BRN GRAY (10YR6/2), BLACK (10YR2/1) FIRECL, RED YELLOW (5YR6/6)

16.2

15.3

FIRECLOUDING; BOTTLE NECK MENDED TO BODY IN PAST, 2 PROBE HOLES ON BODY

BOTTLE-SHORT NECK WITH INCISED PEDESTAL BASE

FLATTENED, EVERTED (FLARED)

5.4

4.1

BULBOUS / SPOOL

SMOOTHED; INCISED, 3 HORIZONTAL LINES

$0.4-0.3$

LOW-WAISTED

15.3

10.0

SMOOTHED; INCISED, NESTED ARCS ABOVE (CREATING SQUARE AROUND BOTTLE NECK WHEN VIEWED FROM ABOVE), NESTED HOOK/SCROLL BELOW

CIRCULAR, PEDESTAL

$9.5 / 2.1$

INCISED, LINE-FILLED TRIANGLES (SIM. TO AFTON)

Huddleston Burial 7, 5/28/1938; Phillips photo 3863 (Huddleston 51); Social Hill-Deceiper phase 


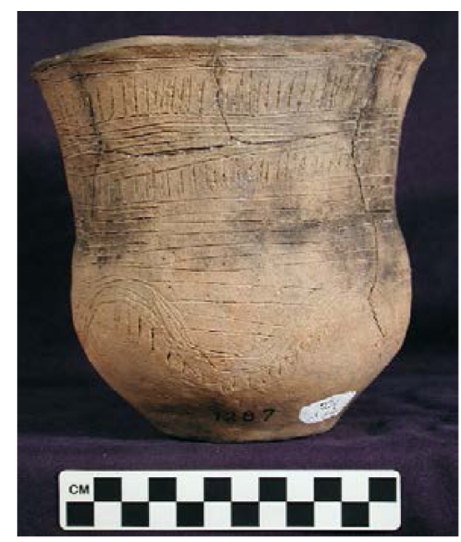

Artifact No. / Old Cat. No. / Site No.

Vessel Form

ARAS/HSU Digital Photo

Type

Decoration

Rim :: Body

Paste

Temper

Core Color (Munsell)

Exterior Color (Munsell)

Interior Color (Munsell)

Vessel Overall Height $(\mathrm{cm})$

Vessel Overall Weight (g)

Vessel Maximum Diameter (cm)

Volume (liters)

Usewear/Sooting/Condition

Shape/Description

Lip Treatment/Shape/Angle/Thickness Orifice Exterior Diameter $(\mathrm{cm})$

Rim/Neck Height $(\mathrm{cm})$

Neck Mid/Base Exterior Diameter $(\mathrm{cm})$

Rim/Neck Shape/Angle

Rim/Neck Surface/Decorative Treatment

Rim/Neck/Body Thickness (cm)

Body Shape

Body Maximum Diameter (cm)

Body Height $(\mathrm{cm})$

Body Surface/Decorative Treatment

Base Shape/Curvature

Base Diameter/Height $(\mathrm{cm})$

Base Surface/Decorative Treatment

Appendages/Handles

Notes
5-32 / 1287 / 3CL81

jar, tall rim

3930

Military Road Incised

incised/punctated

Beloit 1 :: Bard 30 (type)

HARD, SILTY, COMPACT

GROG + SHELL (SOME LEACHED), SPARSE

VERY DARK GRAY (7.5YR3/1)

DK GRAY (10YR4/1), LT YELLOW BROWN (10YR6/4), RED YELLOW (5YR6/6)

VERY DARK GRAY (10YR3/1), YELLOWISH BROWN (10YR5/4), GRAY (10YR5/1)

16.6

696

15.4

FIRECLOUDING, TRACE SOOT EXTERIOR, BLACKENED INTERIOR; RIM REATTACHED TO BODY WITH GLUE, LIP CHIPPED, CRACK IN BODY; 1287 INKED NEAR BASE; HEAVY VESSEL

TALL-RIM JAR WITH FLARED AND FLATTENED LIP, SUBGLOBULAR BODY, AND FLAT BASE

FLATTENED, EVERTED (FLARED)

15.4

7.0

CONCAVE, OUTSLANTED

SMOOTHED; ROWS OF VERTICAL PUNCTATES ALTERNATING WITH IRREGULAR HORIZONTAL LINES

$0.5-0.6$

SUBGLOBULAR

13.4

9.6

SMOOTHED; INCISED \& PUNCTATED, HORIZONTAL AND MEANDERING LINES ABOVE MEANDERING VERTICAL PUNCTATES

CIRCULAR, FLAT

7.2

Huddleston Burial 12, 5/30-5/31/1938; Phillips photo 3913 (Huddleston 60); Mid-Ouachita phase 
Artifact No. / Old Cat. No. / Site No.

Vessel Form

ARAS/HSU Digital Photo

Type

Decoration

Rim :: Body

Paste

Temper

Core Color (Munsell)

Exterior Color (Munsell)

Interior Color (Munsell)

Vessel Overall Height $(\mathrm{cm})$

Vessel Overall Weight (g)

Vessel Maximum Diameter $(\mathrm{cm})$

Volume (liters)

Usewear/Sooting/Condition

Shape/Description

Lip Treatment/Shape/Angle/Thickness

Orifice Exterior Diameter $(\mathrm{cm})$

Rim/Neck Height (cm)

Neck Mid/Base Exterior Diameter (cm)

Rim/Neck Shape/Angle

Rim/Neck Surface/Decorative Treatment

Rim/Neck/Body Thickness (cm)

Body Shape

Body Maximum Diameter $(\mathrm{cm})$

Body Height $(\mathrm{cm})$

Body Surface/Decorative Treatment

Base Shape/Curvature

Base Diameter/Height $(\mathrm{cm})$

Base Surface/Decorative Treatment

Appendages/Handles

Notes
14-20 / 1340 / 3CL81

effigy bowl

3739

appliqued handles

HARD, SILTY, COMPACT

GROG \& SHELL (LEACHED), FINE \& ABUNDANT

VERY DARK GRAYISH BROWN (10YR3/2)

VERY DARK GRAYISH BROWN (10YR3/2), BLACK (10YR2/1), BROWN (10YR5/3)

VERY DARK GRAYISH BROWN (10YR3/2), BROWN (10YR5/3), GRAYISH BROWN (10YR5/2)

13.5

547

24.5

FIRECLOUDING; BODY AND BASE MODERATELY WORN; VESSEL RECONSTRUCTED WITH GLUE, SEVERAL FRAGMENTS MISSING FROM LOWER BODY/BASE; 1340 INKED

SIMPLE BOWL; TWO APPLIQUED HANDLES/NODES, ABSTRACT ANIMALBIRD HEAD/TAIL

FLATTENED, NONE (DIRECT RIM)

24.5

CONTINUOUS WITH BODY

STRAIGHT, OUTSLANTED

SMOOTHED \& BURNISHED

\section{5}

CONICAL

20.4

13.5

SMOOTHED \& BURNISHED

CIRCULAR, FLATTENED

11.7

2 APPLIQUE NODES FOR EFFIGY HEAD AND TAIL

Huddleston Burial 1, 5/14/1938; Phillips photo 3904 (Huddleston 44); prev. identified as 3CL40 but sketch in Huddleston notes fits Stanford description; Mid-Ouachita phase; publ. Trubitt 2017 


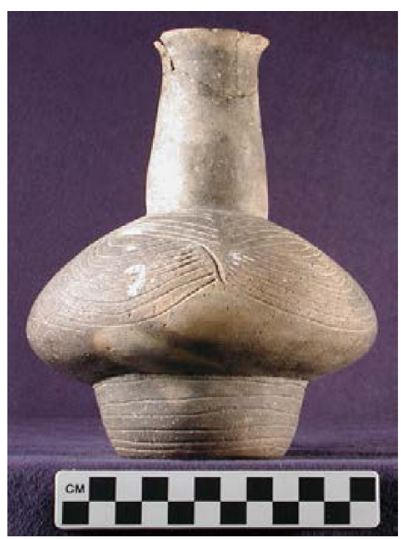

Artifact No. / Old Cat. No. / Site No.

Vessel Form

ARAS/HSU Digital Photo

Type

Decoration

Rim :: Body

Paste

Temper

Core Color (Munsell)

Exterior Color (Munsell)

Interior Color (Munsell)

Vessel Overall Height $(\mathrm{cm})$

Vessel Overall Weight (g)

Vessel Maximum Diameter $(\mathrm{cm})$

Volume (liters)

Usewear/Sooting/Condition

Shape/Description

Lip Treatment/Shape/Angle/Thickness

Orifice Exterior Diameter $(\mathrm{cm})$

Rim/Neck Height (cm)

Neck Mid/Base Exterior Diameter $(\mathrm{cm})$

Rim/Neck Shape/Angle

Rim/Neck Surface/Decorative Treatment

Rim/Neck/Body Thickness (cm)

Body Shape

Body Maximum Diameter $(\mathrm{cm})$

Body Height $(\mathrm{cm})$

Body Surface/Decorative Treatment

Base Shape/Curvature

Base Diameter/Height $(\mathrm{cm})$

Base Surface/Decorative Treatment

Appendages/Handles

Notes
6-1/1243/3CL82

bottle

681

Keno Trailed var. Curtis

incised

plain :: Belhaven 17

HARD, COMPACT, SMOOTH

SHELL (SOME LEACHED), MEDIUM \& ABUNDANT

PALE BROWN (10YR6/3), GRAY (10YR5/1), REDDISH YELLOW (5YR7/6)

DARK GRAYISH BROWN (10YR4/2), VERY PALE BROWN (10YR7/4)

20.6

14.5

FIRECLOUDING; VERY HEAVY VESSEL, INTACT EXCEPT FOR REPAIRED LIP; 1243 INKED ON LOWER BODY

BOTTLE-LONG NECK, INCISED BODY, WITH PEDESTAL BASE

ROUNDED, EVERTED (FLARED)

5.1

8.6

BULBOUS / SPOOL

SMOOTHED, PLAIN

$0.5-0.6$

LOW-WAISTED

14.5

7.9

SMOOTHED \& BURNISHED; INCISED, NESTED ARCS ABOVE (CREATING SQUARE AROUND BOTTLE NECK WHEN VIEWED FROM ABOVE, NESTED SEMICIRCLES BELOW

CIRCULAR, PEDESTAL

$8.2 / 4.1$

6 INCISED HORIZONTAL LINES AROUND PEDESTAL (SIM. TO BATES)

Huddleston Grave 1, 4/9/1939; Phillips photo 3922 (Huddleston 179); Social Hill phase 
JEC Hodges Collection, 77-1

Artifact No. / Old Cat. No. / Site No.

Vessel Form

ARAS/HSU Digital Photo

Type

Decoration

Rim :: Body

Paste

Temper

Core Color (Munsell)

Exterior Color (Munsell)

Interior Color (Munsell)

Vessel Overall Height $(\mathrm{cm})$

Vessel Overall Weight (g)

Vessel Maximum Diameter $(\mathrm{cm})$

Volume (liters)

Usewear/Sooting/Condition

Shape/Description

Lip Treatment/Shape/Angle/Thickness Orifice Exterior Diameter $(\mathrm{cm})$

Rim/Neck Height $(\mathrm{cm})$

Neck Mid/Base Exterior Diameter (cm)

Rim/Neck Shape/Angle

Rim/Neck Surface/Decorative Treatment

Rim/Neck/Body Thickness (cm)

Body Shape

Body Maximum Diameter $(\mathrm{cm})$

Body Height $(\mathrm{cm})$

Body Surface/Decorative Treatment

Base Shape/Curvature

Base Diameter/Height $(\mathrm{cm})$

Base Surface/Decorative Treatment

Appendages/Handles

Notes
6-2 / 1348 / 3CL82

bottle

1815

Keno related

incised/trailed

plain :: Bellarmine 9 (sim.)

HARD, COMPACT

GROG + SHELL (LEACHED), COARSE \& ABUNDANT

STRONG BROWN (7.5YR5/8)

DK GRAY BROWN (10YR4/2), BROWN (10YR5/3), REDDISH YELLOW (5YR6/8)

REDDISH YELLOW (5YR6/8), BROWN (10YR5/3)

17.4

278

11.3

FIRECLOUDING; VESSEL RECONSTRUCTED WITH GLUE; MISSING ABOUT 15\%

\section{SMALL CARINATED BOTTLE WITH LONG NECK}

FLATTENED, EVERTED (FLARED)

3.8

8.4

VERTICAL, SLIGHT SPOOL

SMOOTHED \& BURNISHED, PLAIN

0.5

BICONICAL

11.3

9.2

SMOOTHED; INCISED/TRAILED, NESTED ARCHES ON UPPER BODY

CIRCULAR, CONCAVE

4.5

Huddleston Grave 5, 4/22/1939; Phillips photo 3875 (Huddleston 195); Social Hill phase 
Artifact No. / Old Cat. No. / Site No.

Vessel Form

ARAS/HSU Digital Photo

Type

Decoration

Rim :: Body

Paste

Temper

Core Color (Munsell)

Exterior Color (Munsell)

Interior Color (Munsell)

Vessel Overall Height $(\mathrm{cm})$

Vessel Overall Weight (g)

Vessel Maximum Diameter (cm)

Volume (liters)

Usewear/Sooting/Condition

Shape/Description

Lip Treatment/Shape/Angle/Thickness

Orifice Exterior Diameter $(\mathrm{cm})$

Rim/Neck Height (cm)

Neck Mid/Base Exterior Diameter $(\mathrm{cm})$

Rim/Neck Shape/Angle

Rim/Neck Surface/Decorative Treatment

Rim/Neck/Body Thickness (cm)

Body Shape

Body Maximum Diameter (cm)

Body Height $(\mathrm{cm})$

Body Surface/Decorative Treatment

Base Shape/Curvature

Base Diameter/Height (cm)

Base Surface/Decorative Treatment

Appendages/Handles

Notes

\section{6-3 / 1367 / 3CL82}

bowl, carinated, compound

K8216

Belcher Engraved

engraved

Central or Ezra :: plain

Compact, Hard, Smooth

Shell, medium, abundant

BLACK (7.5YR2.5/1), DARK GRAY (7.5YR4/1), PINKISH WHITE (5YR8/2)

$\operatorname{BLACK}(5 Y R 2.5 / 1)$

7.3

298

18.6

1.0

edges of base roughened; complete vessel, small probe hole in lower body, small crack at rim; 1367 inked on lower body

Compound, carinated bowl

Burnished, Rounded, None, 0.2

18.6 upper, 15.0 lower

1.7 upper, 0.8 lower

upper straight/outslanting, lower straight/inslanting

Burnished, upper - plain, lower- engraved, panels of vertical lines and horizontal ticked lines- pattern repeats 2 times - sim to var. Belcher but upper rim is plain

$0.4-0.3$

Convex

15.5

4.8

Burnished; Plain

Circular/Concave

3.6

Burnished/Plain

small tabular "handles" or nodes on lower edge of lower rim, one on each side of vessel

Huddleston Grave 3, 4/10/1939; Phillips photo 3910 (Huddleston 186); Deceiper phase 
Artifact No. / Old Cat. No. / Site No.

Vessel Form

ARAS/HSU Digital Photo

Type

Decoration

Rim :: Body

Paste

Temper

Core Color (Munsell)

Exterior Color (Munsell)

Interior Color (Munsell)

Vessel Overall Height (cm)

Vessel Overall Weight (g)

Vessel Maximum Diameter (cm)

Volume (liters)

Usewear/Sooting/Condition

Shape/Description

Lip Treatment/Shape/Angle/Thickness Orifice Exterior Diameter (cm)

Rim/Neck Height (cm)

Neck Mid/Base Exterior Diameter (cm)

Rim/Neck Shape/Angle

Rim/Neck Surface/Decorative Treatment

Rim/Neck/Body Thickness (cm)

Body Shape

Body Maximum Diameter (cm)

Body Height $(\mathrm{cm})$

Body Surface/Decorative Treatment

Base Shape/Curvature

Base Diameter/Height $(\mathrm{cm})$

Base Surface/Decorative Treatment

Appendages/Handles

Notes
6-4 / 1355 / 3CL82

jar, short rim

1136

Karnack Brushed-Incised var. Fish Bayou

incised

plain :: Abraham 1

MEDIUM-SOFT, COMPACT

SHELL (SOME LEACHED), MEDIUM \& ABUNDANT

BLACK (10YR2/1), V DK GRAY BRN (10YR3/2), BROWN (7.5YR5/4), FIRECLD

BLACK (10YR2/1), BROWN (7.5YR5/4)

13.8

370

16.8

HEAVY SOOT RESIDUE ON EXTERIOR LOWER BODY/NECK/RIM, ALSO INSIDE RIM, FIRECLOUDING; VESSEL RECONSTRUCTED WITH GLUE AND CLAY/PLASTER IN PAST, PROBE HOLE IN SIDE; 1355 INKED ON RIM

SHORT RIM JAR, GLOBULAR BODY, ROUND BASE, FLARED RIM, WIDE INCISED/TRAILED LINES

ROUNDED, SL. EVERTEED (FLARED)

16.0

2.0

STRAIGHT, OUTSLANTED

SMOOTHED, PLAIN

$0.6-0.3$

GLOBULAR

16.8

11.8

SMOOTHED; INCISED/TRAILED, VERTICAL WIDE LINES FROM NECK TO BASE

UNDISTINGUISHED, CONVEX

6.0

Huddleston Grave 4, 4/10/1939; Phillips photo 3937 (Huddleston 190); Deceiper phase 
Artifact No. / Old Cat. No. / Site No.

Vessel Form

ARAS/HSU Digital Photo

Type

Decoration

Rim :: Body

Paste

Temper

Core Color (Munsell)

Exterior Color (Munsell)

Interior Color (Munsell)

Vessel Overall Height $(\mathrm{cm})$

Vessel Overall Weight (g)

Vessel Maximum Diameter $(\mathrm{cm})$

Volume (liters)

Usewear/Sooting/Condition

Shape/Description

Lip Treatment/Shape/Angle/Thickness

Orifice Exterior Diameter $(\mathrm{cm})$

Rim/Neck Height (cm)

Neck Mid/Base Exterior Diameter $(\mathrm{cm})$

Rim/Neck Shape/Angle

Rim/Neck Surface/Decorative Treatment

Rim/Neck/Body Thickness (cm)

Body Shape

Body Maximum Diameter (cm)

Body Height $(\mathrm{cm})$

Body Surface/Decorative Treatment

Base Shape/Curvature

Base Diameter/Height $(\mathrm{cm})$

Base Surface/Decorative Treatment

Appendages/Handles

Notes
6-5/ 1351 / 3CL82

bowl, simple

K8342

plain

Compact, Hard, Smooth

Shell (leached), fine to medium, abundant

$\operatorname{BLACK}(7.5 Y R 2.5 / 1)$

BROWN (7.5YR5/3-5/4), DARK GRAY (7.5YR4/1)

PINKISH GRAY (7.5YR6/2), VERY DARK GRAY (7.5YR3/1), BLACK (7.5YR2.5/1)

8.0

482

25.6

Light roughening on exterior base; fireclouding; partial vessel reconstructed and partly plastered in past, several sherds missing from rim/body; 1351 inked on base, remains of paper tag on interior

Bowl - open, shallow

Smoothed, Rounded, Everted, 0.3

25.6

Concave, Outslanted, Continuous with body

Burnished, Plain

$0.4-0.5$

Convex

25.6

8.0

Burnished; Plain

Undistinguished/Convex

Smoothed/Plain

Huddleston Grave 5, 4/22/1939; Phillips photo 3985 (Huddleston 193); Social Hill phase 
Artifact No. / Old Cat. No. / Site No.

Vessel Form

ARAS/HSU Digital Photo

Type

Decoration

Rim :: Body

Paste

Temper

Core Color (Munsell)

Exterior Color (Munsell)

Interior Color (Munsell)

Vessel Overall Height $(\mathrm{cm})$

Vessel Overall Weight (g)

Vessel Maximum Diameter $(\mathrm{cm})$

Volume (liters)

Usewear/Sooting/Condition

Shape/Description

Lip Treatment/Shape/Angle/Thickness Orifice Exterior Diameter $(\mathrm{cm})$

Rim/Neck Height $(\mathrm{cm})$

Neck Mid/Base Exterior Diameter $(\mathrm{cm})$

Rim/Neck Shape/Angle

Rim/Neck Surface/Decorative Treatment

Rim/Neck/Body Thickness (cm)

Body Shape

Body Maximum Diameter $(\mathrm{cm})$

Body Height $(\mathrm{cm})$

Body Surface/Decorative Treatment

Base Shape/Curvature

Base Diameter/Height $(\mathrm{cm})$

Base Surface/Decorative Treatment

Appendages/Handles

Notes
6-6/1362 / 3CL82

bowl, simple

621

Keno/Wallace?

incised

plain :: Buffalo 5

SOFT, SILTY, COMPACT

SHELL, COARSE \& ABUNDANT

DARK GRAY, VERY PALE BROWN, GRAY

DARK GRAY, VERY PALE BROWN, BLACK

16.0

30.7

FIRECLOUDING; SOME SURFACE SPALLING/EROSION; RECONSTRUCTED IN PAST; 1362 INKED NEAR BASE

WIDE 'HELMET-SHAPED' BOWL WITH OUTSLANTING RIM AND ROUND CONVEX BASE

FLATTENED, EVERTED (FLARED)

30.7

3.7

STRAIGHT, OUTSLANTED

SMOOTHED \& BURNISHED, PLAIN

$0.7-0.7$

CONVEX

26.9

12.3

SMOOTHED \& BURNISHED; INCISED, VERY BROAD CURVILINEAR TRAILING, NESTED SEMICIRCLES

UNDISTINGUISHED, CONVEX

Huddleston Grave 3, 4/10/1939; Phillips photo 3993 (Huddleston 189); Deceiper phase 


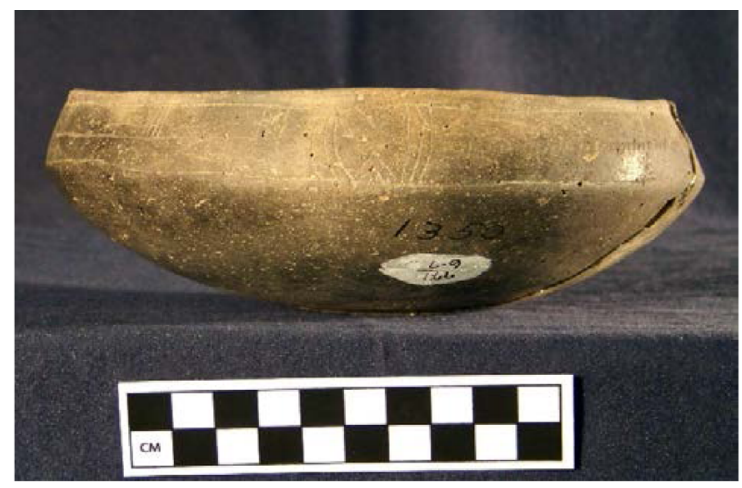

Artifact No. / Old Cat. No. / Site No.

Vessel Form

ARAS/HSU Digital Photo

Type

Decoration

Rim :: Body

Paste

Temper

Core Color (Munsell)

Exterior Color (Munsell)

Interior Color (Munsell)

Vessel Overall Height $(\mathrm{cm})$

Vessel Overall Weight (g)

Vessel Maximum Diameter $(\mathrm{cm})$

Volume (liters)

Usewear/Sooting/Condition

Shape/Description

Lip Treatment/Shape/Angle/Thickness Orifice Exterior Diameter (cm)

Rim/Neck Height $(\mathrm{cm})$

Neck Mid/Base Exterior Diameter (cm) Rim/Neck Shape/Angle

Rim/Neck Surface/Decorative Treatment

Rim/Neck/Body Thickness (cm)

Body Shape

Body Maximum Diameter (cm)

Body Height $(\mathrm{cm})$

Body Surface/Decorative Treatment

Base Shape/Curvature

Base Diameter/Height (cm)

Base Surface/Decorative Treatment

Appendages/Handles

Notes
6-7 / 1350 / 3CL82

bowl, carinated

K5413

Cook Engraved

engraved

Ezra $12::$ plain

Compact, Hard, Smooth

Shell, leached, medium \& abundant + grog

BROWN (7.5YR5/2)

DARK GRAY (7.5YR4/1)

6.5

1

18.0

1.0

Firecloouding; partial vessel, reassembled in past, large rim/body sherd missing; 1350 inked on body/base

Bowl, Carinated

burnished, rounded, 0.5

16.5

2.5

Convex, Inslanted

burnished, 2 engraved horiz lines, one under lip, one at shoulder, central motif $x 4$ with alternating horiz ticked line and segmented ladder

0.5

Convex

18.0

4.0

Burnished; Plain

Undistinguished/Convex

Smoothed/Plain

No rim tabs

Huddleston Grave 5, 4/22/1939; Phillips photo 3930 (Huddleston 192); Social Hill phase 
Artifact No. / Old Cat. No. / Site No.

Vessel Form

ARAS/HSU Digital Photo

Type

Decoration

$\operatorname{Rim}::$ Body

Paste

Temper

Core Color (Munsell)

Exterior Color (Munsell)

Interior Color (Munsell)

Vessel Overall Height (cm)

Vessel Overall Weight (g)

Vessel Maximum Diameter $(\mathrm{cm})$

Volume (liters)

Usewear/Sooting/Condition

Shape/Description

Lip Treatment/Shape/Angle/Thickness

Orifice Exterior Diameter $(\mathrm{cm})$

Rim/Neck Height (cm)

Neck Mid/Base Exterior Diameter (cm)

Rim/Neck Shape/Angle

Rim/Neck Surface/Decorative Treatment

Rim/Neck/Body Thickness (cm)

Body Shape

Body Maximum Diameter (cm)

Body Height $(\mathrm{cm})$

Body Surface/Decorative Treatment

Base Shape/Curvature

Base Diameter/Height (cm)

Base Surface/Decorative Treatment

Appendages/Handles

Notes
$6-8 / 1354$ / 3CL82

bowl, carinated

N13990

Means Engraved

engraved

Enos 19 (type) :: plain

Compact, Hard, Smooth

Shell, leached \& Grog, sparse and fine

REDDISH BROWN (2.5YR5/3) (CORE MODE B)

LT BROWN GRAY (10YR6/2), LT GRAY (10YR7/2), REDDISH BLACK (2.5YR2.5/1)

WEAK RED (2.5YR5/2), REDDISH GRAY (2.5YR5/1), DARK REDDISH GRAY (2.5YR4/1)

7.0

289

16.7

1.0

Fireclouding; partial vessel reconstructed with glue in past, missing sherds from base, body, chips; 1354 inked on base of body, residue of old sticker on mid-body

Carinated Bowl

burnished, round, sl. everted, 0.3; engraved ticked lines/punctates

16.7

3.0

Straight, Carinated/inslanted

Burnished, Engraved, 4 panels of diagonal line with tick marks surrounding squares with 3 horizontal line between

0.3

Conical

16.7

4.0

Burnished; Plain

Circular/Flat

6.0

Burnished/Plain

Huddleston Grave 2, 4/9/1939; Phillips photo 3891 (Huddleston 185); previous illustration Suhm \& Jelks 1962:Plate 53J; Social Hill - Deceiper phase 
JEC Hodges Collection, 77-1

Artifact No. / Old Cat. No. / Site No.

Vessel Form

ARAS/HSU Digital Photo

Type

Decoration

Rim :: Body

Paste

Temper

Core Color (Munsell)

Exterior Color (Munsell)

Interior Color (Munsell)

Vessel Overall Height $(\mathrm{cm})$

Vessel Overall Weight $(\mathrm{g})$

Vessel Maximum Diameter $(\mathrm{cm})$

Volume (liters)

Usewear/Sooting/Condition

Shape/Description

Lip Treatment/Shape/Angle/Thickness

Orifice Exterior Diameter $(\mathrm{cm})$

Rim/Neck Height (cm)

Neck Mid/Base Exterior Diameter $(\mathrm{cm})$

Rim/Neck Shape/Angle

Rim/Neck Surface/Decorative Treatment

Rim/Neck/Body Thickness (cm)

Body Shape

Body Maximum Diameter (cm)

Body Height $(\mathrm{cm})$

Body Surface/Decorative Treatment

Base Shape/Curvature

Base Diameter/Height $(\mathrm{cm})$

Base Surface/Decorative Treatment

Appendages/Handles

Notes
6-9 / 1364 / 3CL82

jar, short rim

N14000

Karnack Brushed-Incised var. Midway

incised

plain :: Abraham 1 (type)

Compact, Hard, Smooth

Shell, coarse \& abundant

RED BLACK (2.5YR2.5/1), RED YELLOW (5YR6/8), PINKISH GRAY (7.5YR6/2)

PINKISH GRAY (7.5YR7/2), BLACK (7.5YR2.5/1)

11.0

279

11.2

0.6

Light roughening ext base; slight fireclouding; intact vessel; 1364 inked on base

JAR

Burnished, rounded, sl. everted, 0.4

10.2

1.0

Straight, Outslanted

Burnished

$0.3-0.4$

Ovoid

11.2

10.0

Smoothed; Incised, vertical incising from rim to base, wide line wet paste incising

Undistinguished/Convex

6.5

Smoothed/Plain

Huddleston Grave 3, 4/10/1939; Phillips photo 3874 (Huddleston 187); Deceiper phase 
Artifact No. / Old Cat. No. / Site No.

Vessel Form

ARAS/HSU Digital Photo

Type

Decoration

Rim :: Body

Paste

Temper

Core Color (Munsell)

Exterior Color (Munsell)

Interior Color (Munsell)

Vessel Overall Height (cm)

Vessel Overall Weight (g)

Vessel Maximum Diameter (cm)

Volume (liters)

Usewear/Sooting/Condition

Shape/Description

Lip Treatment/Shape/Angle/Thickness

Orifice Exterior Diameter $(\mathrm{cm})$

Rim/Neck Height (cm)

Neck Mid/Base Exterior Diameter (cm)

Rim/Neck Shape/Angle

Rim/Neck Surface/Decorative Treatment

Rim/Neck/Body Thickness (cm)

Body Shape

Body Maximum Diameter (cm)

Body Height $(\mathrm{cm})$

Body Surface/Decorative Treatment

Base Shape/Curvature

Base Diameter/Height (cm)

Base Surface/Decorative Treatment

Appendages/Handles

Notes
6-10 / 1358 / 3CL82

jar, tall rim

N10096

Foster Trailed-Incised var. Foster

incised

Alfred 2 (type) :: Baker 16 (type)

Compact, Hard, Smooth

Shell, leached, coarse \& abundant

BLACK (7.5YR2.5/1)

V DK GRAY (10YR3/1), RED YELLOW (7.5YR6/6), LT BROWN GRAY (10YR6/2)

$\operatorname{BLACK}(7.5 Y R 2.5 / 1)$

18.2

721

17.9

Encrustation interior rim, fireclouding; partial vessel, reconstructed, missing one node and sev pcs from lip/rim; 1358 inked near base

JAR

Smoothed, flat, sl. everted, 0.4

17.9

8.0

Concave, Outslanted

Smoothed, Incised, 4 rows of Diagonal Slash Incising, bands, unzoned, around $1 \mathrm{~cm}$ slashes, rows, $1 \mathrm{~cm}$ apart

$0.4-0.5$

sub-globular

15.4

10.2

Smoothed; Incised/Trailed; trailed lines $2 \mathrm{~mm}$ wide, $5-6 \mathrm{~mm}$ apart, 3 sets of concentric circles with central 3-part nodes (one missing); design stops $1 \mathrm{~cm}$ above base

circular/flat

6.0

Smoothed/Plain

3 nodes, nodes are 3 -ball applique

Huddleston Grave 2, 4/9/1939; Phillips photo 3959 (Huddleston 180[181]); Social Hill - Deceiper phase 
JEC Hodges Collection, 77-1

Artifact No. / Old Cat. No. / Site No.

Vessel Form

ARAS/HSU Digital Photo

Type

Decoration

Rim :: Body

Paste

Temper

Core Color (Munsell)

Exterior Color (Munsell)

Interior Color (Munsell)

Vessel Overall Height $(\mathrm{cm})$

Vessel Overall Weight (g)

Vessel Maximum Diameter (cm)

Volume (liters)

Usewear/Sooting/Condition

Shape/Description

Lip Treatment/Shape/Angle/Thickness

Orifice Exterior Diameter (cm)

Rim/Neck Height (cm)

Neck Mid/Base Exterior Diameter $(\mathrm{cm})$

Rim/Neck Shape/Angle

Rim/Neck Surface/Decorative Treatment

Rim/Neck/Body Thickness (cm)

Body Shape

Body Maximum Diameter $(\mathrm{cm})$

Body Height $(\mathrm{cm})$

Body Surface/Decorative Treatment

Base Shape/Curvature

Base Diameter/Height $(\mathrm{cm})$

Base Surface/Decorative Treatment

Appendages/Handles

Notes
6-11 / 1369 / 3CL82

bottle

674

Keno Trailed var. Red Hill

incised

plain :: Belhaven 31

SOFT, SILTY, COMPACT

SHELL, LEACHED, FINE \& SPARSE

CM

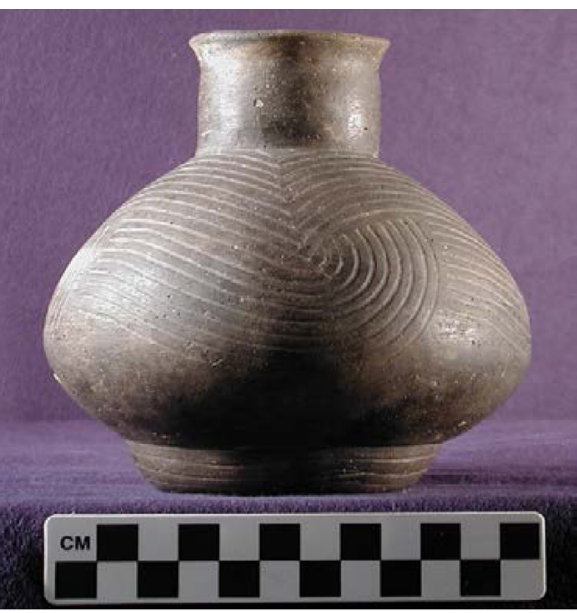

GRAY (10YR5/1), DARK GRAY (10YR4/1), VERY PALE BROWN (10YR8/3)

14.3

13.3

FIRECLOUDING; VESSEL INTACT EXCEPT FOR ONE PROBE HOLE NEAR PEDESTAL AND ANOTHER PROBABLE PROBE HOLE NEAR PEDESTAL

SHORT SQUAT BOTTLE WITH FLARED RIM, SHORT NECK, PEDESTAL BASE

SMOOTHED, FLATTENED, EVERTED (FLARED), THINNED

5.6

3.7

SPOOL

SMOOTHED \& BURNISHED, PLAIN

0.5

LOW-WAIST

13.3

10.6

SMOOTHED \& BURNISHED; INCISED, nested arcs creating square around neck, with nested scrolls on lower body

PEDESTAL

$8.7 / 1.7$

4 INCISED HORIZONTAL LINES AROUND PEDESTAL

probably Huddleston Grave 2, 4/9/1939 (sketch in notes); Phillips photo 3855 (Huddleston 84[184]); Social Hill - Deceiper phase 


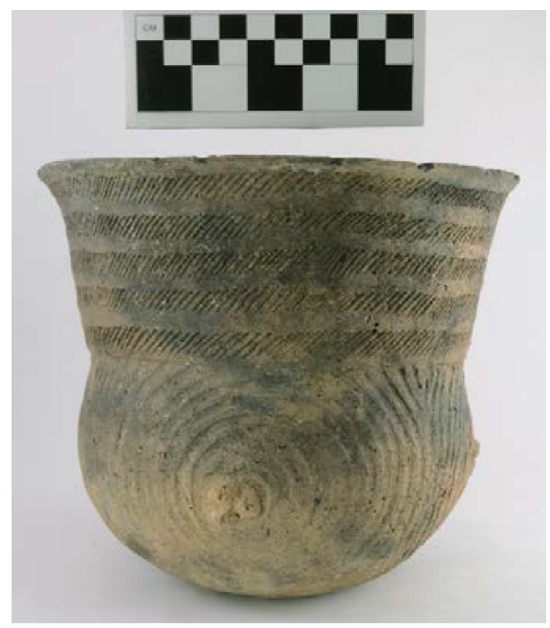

Artifact No. / Old Cat. No. / Site No.

Vessel Form

ARAS/HSU Digital Photo

Type

Decoration

Rim :: Body

Paste

Temper

Core Color (Munsell)

Exterior Color (Munsell)

Interior Color (Munsell)

Vessel Overall Height (cm)

Vessel Overall Weight (g)

Vessel Maximum Diameter $(\mathrm{cm})$

Volume (liters)

Usewear/Sooting/Condition

Shape/Description

Lip Treatment/Shape/Angle/Thickness

Orifice Exterior Diameter $(\mathrm{cm})$

Rim/Neck Height (cm)

Neck Mid/Base Exterior Diameter (cm)

Rim/Neck Shape/Angle

Rim/Neck Surface/Decorative Treatment

Rim/Neck/Body Thickness (cm)

Body Shape

Body Maximum Diameter (cm)

Body Height $(\mathrm{cm})$

Body Surface/Decorative Treatment

Base Shape/Curvature

Base Diameter/Height $(\mathrm{cm})$

Base Surface/Decorative Treatment

Appendages/Handles

Notes
6-12 / 1357 / 3CL82

jar, tall rim

N14010

Foster Trailed-Incised var. Foster

incised

Alfred 6 (type) :: Baker 3 (type)

Compact, Hard, Smooth

Shell (some leached), coarse \& abundant

$\operatorname{PINK}(7.5 Y R 7 / 4)$

V DK GRAY (10YR3/1), PINKISH GRAY (7.5YR7/2), REDDISH YELLOW (7.5YR6/6)

VERY DARK GRAY (5YR3/1), PINKISH GRAY (7.5YR7/2), REDDISH YELLOW (7.5YR6/6)

18.4

752

19.4

3.0

Rim worn away on one side; possible small areas of soot on int rim; vessel is complete, chips to lip; 1357 inked on int rim, remnant of paper tag on interior

Jar, Tall Rim; rim is uneven

Smoothed, Rounded, Sl. Everted, 0.4

19.4

8.2

Straight, Outslanted

Smoothed, Incised, 5 bands of slanted, Incised lines, $1 \mathrm{~cm}$ band of slash inc., $0.5 \mathrm{~cm}$ space betw

$0.4-0.3$

Sub-Globular

15.9

10.2

Smoothed; Incised/Trailed, concentric lines with 3 tri-node clusters - Pattern reports 3 times

Circular/Flat

6.8

Smoothed/Plain

Three tri node clusters at waist of body

Huddleston Grave 2, 4/9/1939; Phillips photo 3948-49 (Huddleston 181); Social Hill - Deceiper phase 
JEC Hodges Collection, 77-1

Artifact No. / Old Cat. No. / Site No.

Vessel Form

ARAS/HSU Digital Photo

Type

Decoration

Rim :: Body

Paste

Temper

Core Color (Munsell)

Exterior Color (Munsell)

Interior Color (Munsell)

Vessel Overall Height $(\mathrm{cm})$

Vessel Overall Weight (g)

Vessel Maximum Diameter $(\mathrm{cm})$

Volume (liters)

Usewear/Sooting/Condition

Shape/Description

Lip Treatment/Shape/Angle/Thickness

Orifice Exterior Diameter $(\mathrm{cm})$

Rim/Neck Height (cm)

Neck Mid/Base Exterior Diameter $(\mathrm{cm})$

Rim/Neck Shape/Angle

Rim/Neck Surface/Decorative Treatment

Rim/Neck/Body Thickness (cm)

Body Shape

Body Maximum Diameter (cm)

Body Height $(\mathrm{cm})$

Body Surface/Decorative Treatment

Base Shape/Curvature

Base Diameter/Height (cm)

Base Surface/Decorative Treatment

Appendages/Handles

Notes
6-13 / 1359 / 3CL82

jar, tall rim

N13980

Foster Trailed-Incised var. Dobson

incised/brushed

Arlis 3 (type) :: Baker 1 (type)

Compact, Hard, Smooth

Shell (partly leached), fine \& abundant

GRAY (7.5YR5/1)

GRAY (7.5YR6/1-10YR5/1), DARK GRAY (10YR4/1)

VERY DARK GRAYISH BROWN (10YR3/2), REDDISH GRAY (2.5YR5/1)

11.0

310

13.1

0.8

Some int rim sooting, fireclouding; complete vessel except missing rim sherd and lip chipped; 1359 inked on int rim

JAR

Smoothed, Flattened, S1. Everted, 0.3

13.1

4.5

Straight, Outslanted

Smoothed, Incised \& Brushed, top \& bottom band incised slant, Middle band horizontal brushing (sism to Dana 14)

0.4

Sub-Globular

11.2

6.5

Smoothed; Incised/Trailed, wide flat lines, 3 repeats/panels of concentric circles

Circular/Flat

4.0

Smoothed/Plain

no central nodes on body

Huddleston Grave 1, 4/9/1939; Phillips photo 3876 (Huddleston 178); Social Hill phase 
Artifact No. / Old Cat. No. / Site No.

Vessel Form

ARAS/HSU Digital Photo

Type

Decoration

$\operatorname{Rim}::$ Body

Paste

Temper

Core Color (Munsell)

Exterior Color (Munsell)

Interior Color (Munsell)

Vessel Overall Height (cm)

Vessel Overall Weight (g)

Vessel Maximum Diameter $(\mathrm{cm})$

Volume (liters)

Usewear/Sooting/Condition

Shape/Description

Lip Treatment/Shape/Angle/Thickness

Orifice Exterior Diameter (cm)

Rim/Neck Height (cm)

Neck Mid/Base Exterior Diameter (cm)

Rim/Neck Shape/Angle

Rim/Neck Surface/Decorative Treatment

Rim/Neck/Body Thickness (cm)

Body Shape

Body Maximum Diameter $(\mathrm{cm})$

Body Height $(\mathrm{cm})$

Body Surface/Decorative Treatment

Base Shape/Curvature

Base Diameter/Height (cm)

Base Surface/Decorative Treatment

Appendages/Handles

Notes
8-1/1207 / 3CL23

effigy seed jar, quadruped

1211

incised/modeled

SOFT, COARSE, SOME SAND/PEBBLES

GROG, FINE/ABUNDANT

LIGHT YELLOWISH BROWN (10YR6/4)

DK GRAY (10YR4/1), V PALE BROWN (10YR7/3), REDDISH YELLOW (5YR6/6)

21.0

1194

15.2

VESSEL WAS HEAVILY RECONSTRUCTED WITH GLUE AND PLASTER; FRONT LEGS BROKEN

LARGE BULBOUS EFFIGY SEED JAR WITH 4 LEGS; OPENING ON TOP OF THE HEAD; BEAR? EFFIGY

ROUNDED

3.3

4.0

SMOOTHED \& BURNISHED, MODELED HEAD FEATURES (EARS, SNOUT) WITH INCISED EYE CIRCLES, MOUTH LINE, AND 2 PUNCTATES FOR NOSE

$0.7-0.6$

GLOBULAR, OFFSET BETWEEN LEGS (SUPPORTS) AND HEAD (OPENING)

15.2

13.6

SMOOTHED \& BURNISHED; MODELED LEGS ATTACHED TO BASE, FRONT LEGS ATTACHED ONTO BODY

CONVEX

4 LEGS AND TAIL, NOT HOLLOW AS EVIDENCED BY BROKEN APPENDAGES

Huddleston Grave 3, 12/22-30/1938[1937?]; Phillips photo 3946 (Huddleston 38); Mid-Ouachita phase; previous illustration Suhm \& Jelks 1962:Plate 25F; publ. Trubitt 2017 
Artifact No. / Old Cat. No. / Site No.

Vessel Form

ARAS/HSU Digital Photo

Type

Decoration

$\operatorname{Rim}::$ Body

Paste

Temper

Core Color (Munsell)

Exterior Color (Munsell)

Interior Color (Munsell)

Vessel Overall Height $(\mathrm{cm})$

Vessel Overall Weight (g)

Vessel Maximum Diameter $(\mathrm{cm})$

Volume (liters)

Usewear/Sooting/Condition

Shape/Description

Lip Treatment/Shape/Angle/Thickness

Orifice Exterior Diameter $(\mathrm{cm})$

Rim/Neck Height (cm)

Neck Mid/Base Exterior Diameter $(\mathrm{cm})$

Rim/Neck Shape/Angle

Rim/Neck Surface/Decorative Treatment

Rim/Neck/Body Thickness (cm)

Body Shape

Body Maximum Diameter (cm)

Body Height $(\mathrm{cm})$

Body Surface/Decorative Treatment

Base Shape/Curvature

Base Diameter/Height $(\mathrm{cm})$

Base Surface/Decorative Treatment

Appendages/Handles

Notes
$8-2 / 1209 / 3$ CL23

bottle

K8209

Crockett Curvilinear Incised (similar)

engraved

plain :: Elmhurst 1 (type)

Compact, Hard, Smooth, Mica in paste

Grog + shell (leached/voids) fine, sparse

VERY PALE BROWN (10YR8/2), LIGHT RED (10R6/8), BLACK (5YR2.5/1)

REDDISH YELLOW (5YR6/8)

19.5

950

18.0

1.6

Vessel is complete, small chip in lip; light-colored vessel, refired?; 1209 inked on base

Bottle

Smoothed, Rounded, None, 0.7

5.3

8.0

$6.3 / 7.3$

Insloping

Smoothed

0.4

Low Waist

18.0

11.5

Smoothed; Engraved, ticked line scroll, "punctates", crosshatching; pattern repeats 4 times, similar to Crockett C-I design

Undistinguished/convex

Smoothed/Plain

Huddleston Grave 8, 1/14/1938[1939]; Phillips photo 3939 (Huddleston 125); early Mid-Ouachita phase 


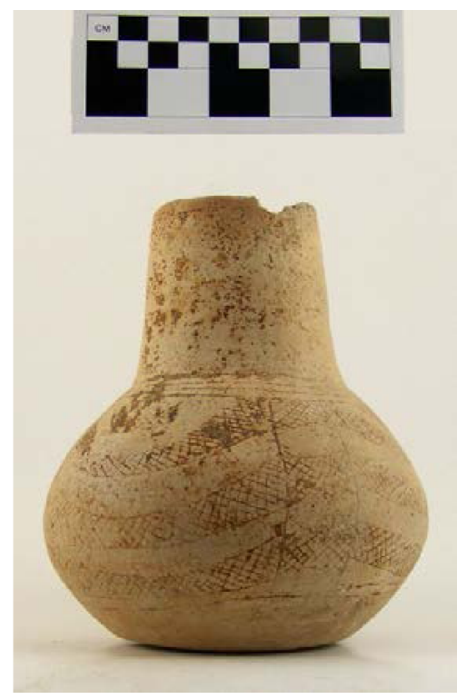

Artifact No. / Old Cat. No. / Site No.

Vessel Form

ARAS/HSU Digital Photo

Type

Decoration

Rim :: Body

Paste

Temper

Core Color (Munsell)

Exterior Color (Munsell)

Interior Color (Munsell)

Vessel Overall Height (cm)

Vessel Overall Weight (g)

Vessel Maximum Diameter (cm)

Volume (liters)

Usewear/Sooting/Condition

Shape/Description

Lip Treatment/Shape/Angle/Thickness

Orifice Exterior Diameter $(\mathrm{cm})$

Rim/Neck Height (cm)

Neck Mid/Base Exterior Diameter (cm)

Rim/Neck Shape/Angle

Rim/Neck Surface/Decorative Treatment

Rim/Neck/Body Thickness (cm)

Body Shape

Body Maximum Diameter (cm)

Body Height $(\mathrm{cm})$

Body Surface/Decorative Treatment

Base Shape/Curvature

Base Diameter/Height (cm)

Base Surface/Decorative Treatment

Appendages/Handles

Notes
8-3 / 1236 / 3CL23

bottle

\section{K8180}

Maddox Engraved?

engraved

plain :: Erie 10, Eric 28 (type)

Compact, Hard, Smooth

Grog, fine, sparse

\section{VERY PALE BROWN (10YR7/4)}

\section{5}

495

12.0

0.6

Roughened base, lip chipped; vessel is complete; light-colored vessel, refired?

\section{Bottle}

Smoothed, Flat, None, 0.5

5.3

6.0

$6.0 / 7.0$

insloping

Smoothed

0.5

Subglobular

12.0

9.5

Smoothed; Engraved, crosshatched bands - 4 lines at top of body $5 \mathrm{~mm}$ apart, three vertical panels divide body, crosshatching not continuous across panels; trace of red pigment in design

Circular/Flat

7.0

Smoothed/Plain

Huddleston Grave 5, 3/19/1938; Phillips photo 3861 (Huddleston 42); early Mid-Ouachita phase 
JEC Hodges Collection, 77-1

Artifact No. / Old Cat. No. / Site No.

Vessel Form

ARAS/HSU Digital Photo

Type

Decoration

Rim :: Body

Paste

Temper

Core Color (Munsell)

Exterior Color (Munsell)

Interior Color (Munsell)

Vessel Overall Height $(\mathrm{cm})$

Vessel Overall Weight (g)

Vessel Maximum Diameter (cm)

Volume (liters)

Usewear/Sooting/Condition

Shape/Description

Lip Treatment/Shape/Angle/Thickness

Orifice Exterior Diameter $(\mathrm{cm})$

Rim/Neck Height $(\mathrm{cm})$

Neck Mid/Base Exterior Diameter (cm)

Rim/Neck Shape/Angle

Rim/Neck Surface/Decorative Treatment

Rim/Neck/Body Thickness (cm)

Body Shape

Body Maximum Diameter (cm)

Body Height (cm)

Body Surface/Decorative Treatment

Base Shape/Curvature

Base Diameter/Height $(\mathrm{cm})$

Base Surface/Decorative Treatment

Appendages/Handles

Notes
$8-4 / 1204 / 3$ CL23

bowl, simple

K5070, K5169

Hempstead Engraved?

engraved

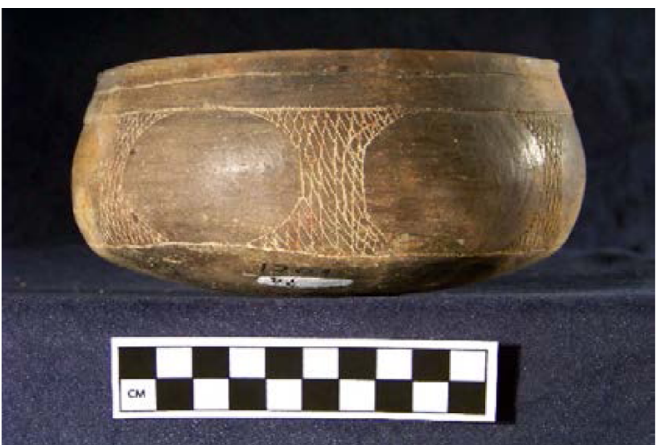

Erie $1::$ Elmira 2

Compact, Hard, Smooth

grog, fine to medium, sparse to medium

PINKISH GRAY (7.5YR7/2), VERY DARK GRAY (7.5YR3/1)

BLACK (7.5YR2.5/1), PINK (7.5YR7/4), REDDISH YELLOW (5YR6/6)

BLACK (7.5YR2.5/1), PINKISH GRAY (7.5YR7/2)

8.5

514

16.4

1.1

Vessel reassembled with glue, several scars from probe; 1204 inked on base

Bowl, simple

Burnished, thinned, sl everted, 0.3

14.9

1.9

Convex, Inslanted

Burnished-eroded, Engraved, 1 engraved line 6-8mm below lip

$0.5-0.6$

Low-waist, continuous rim/body/base

16.4

7.0

Burnished; Engraved, crosshatched background, plain balls/ovals, squashed, line on top and bottom of pattern, traces of red pigment in engraved areas

Undistinguished/ convex

Burnished/Plain

Huddleston Grave 8, 1/14/1938[1939]; Phillips photo 3918 (Huddleston 122); early Mid-Ouachita phase 
JEC Hodges Collection, 77-1

Artifact No. / Old Cat. No. / Site No.

Vessel Form

ARAS/HSU Digital Photo

Type

Decoration

$\operatorname{Rim}:$ : Body

Paste

Temper

Core Color (Munsell)

Exterior Color (Munsell)

Interior Color (Munsell)

Vessel Overall Height (cm)

Vessel Overall Weight (g)

Vessel Maximum Diameter $(\mathrm{cm})$

Volume (liters)

Usewear/Sooting/Condition

Shape/Description

Lip Treatment/Shape/Angle/Thickness

Orifice Exterior Diameter (cm)

Rim/Neck Height (cm)

Neck Mid/Base Exterior Diameter (cm)

Rim/Neck Shape/Angle

Rim/Neck Surface/Decorative Treatment

Rim/Neck/Body Thickness (cm)

Body Shape

Body Maximum Diameter $(\mathrm{cm})$

Body Height $(\mathrm{cm})$

Body Surface/Decorative Treatment

Base Shape/Curvature

Base Diameter/Height (cm)

Base Surface/Decorative Treatment

Appendages/Handles

Notes
8-5/ 1230/3CL23

bowl, simple

584

Hempstead Engraved

engraved

Erie 2 or 1 ?:: Elmira 8

HARD, FINE, COMPACT

GROG \& CLAYGRIT, ABUNDANT

LIGHT GRAY

DARK REDDISH BROWN, BLACK FIRECLOUDS, REDDISH YELLOW

BLACK

10.1

20.2

FIRECLOUDING; CHIPS TO LIP; 1230 INKED NEAR BASE

WIDE BOWL WITH ROUND CONVEX BASE; TALL INSLANTING TWO-FIELDED RIM; LIP IS SLIGHTLY THINNED AND FLATTENED

THINNED, FLATTENED

19.4

1.9

CONVEX, INSLANTED

SMOOTHED \& BURNISHED, ENGRAVED-HORIZONTAL ENGRAVED LINE BELOW LIP 0.6

LOW-WAIST, CONTINUOUS RIM/BODY/BASE

20.2

3.6

SMOOTHED \& BURNISHED; ENGRAVED CROSSHATCHED TRIANGLES /PLAIN DIAMONDS, HORIZ LINES ABOVE AND BELOW

UNDISTINGUISHED/CONVEX

BURNISHED/PLAIN

Huddleston Grave 8, 1/14/1938[1939]; Phillips photo 3960 (Huddleston 124); early Mid-Ouachita phase 


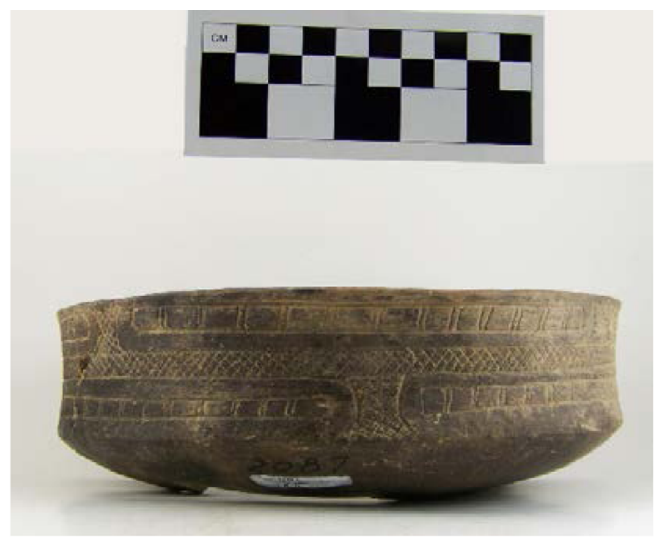

Artifact No. / Old Cat. No. / Site No.

Vessel Form

ARAS/HSU Digital Photo

Type

Decoration

Rim :: Body

Paste

Temper

Core Color (Munsell)

Exterior Color (Munsell)

Interior Color (Munsell)

Vessel Overall Height $(\mathrm{cm})$

Vessel Overall Weight (g)

Vessel Maximum Diameter (cm)

Volume (liters)

Usewear/Sooting/Condition

Shape/Description

Lip Treatment/Shape/Angle/Thickness

Orifice Exterior Diameter $(\mathrm{cm})$

Rim/Neck Height (cm)

Neck Mid/Base Exterior Diameter $(\mathrm{cm})$

Rim/Neck Shape/Angle

Rim/Neck Surface/Decorative Treatment

Rim/Neck/Body Thickness (cm)

Body Shape

Body Maximum Diameter (cm)

Body Height $(\mathrm{cm})$

Body Surface/Decorative Treatment

Base Shape/Curvature

Base Diameter/Height $(\mathrm{cm})$

Base Surface/Decorative Treatment

Appendages/Handles

Notes
8-6 / 2087 / 3CL23

bowl, carinated

K8251

Friendship Engraved var. Freeman

engraved

Elaine 21 :: plain

Compact, Hard, Smooth

Grog, coarse

DARK REDDISH GRAY (10R3/1-4/1), VERY PALE BROWN (10YR8/2)

DARK GRAY (10YR4/1)

7.5

594

21.1

Encrustations; partial vessel, reconstructed with glue, missing most of base; 2087 inked body

Bowl, carinated

Flattened, sl. everted, 0.4

21.1

5.0

Concave, Outslanted

Burnished-eroded, Engraved, horizontal elongated ovals (segmented) with engraved crosshatching in between

0.5

Convex, continuous with base

21.1

2.5

Burnished; Plain

Undistinguished / mostly missing

Huddleston Grave 12, 12/9/1939; Mid-Ouachita phase 
Artifact No. / Old Cat. No. / Site No.

Vessel Form

ARAS/HSU Digital Photo

Type

Decoration

Rim :: Body

Paste

Temper

Core Color (Munsell)

Exterior Color (Munsell)

Interior Color (Munsell)

Vessel Overall Height (cm)

Vessel Overall Weight (g)

Vessel Maximum Diameter (cm)

Volume (liters)

Usewear/Sooting/Condition

Shape/Description

Lip Treatment/Shape/Angle/Thickness

Orifice Exterior Diameter $(\mathrm{cm})$

Rim/Neck Height (cm)

Neck Mid/Base Exterior Diameter (cm)

Rim/Neck Shape/Angle

Rim/Neck Surface/Decorative Treatment

Rim/Neck/Body Thickness (cm)

Body Shape

Body Maximum Diameter (cm)

Body Height $(\mathrm{cm})$

Body Surface/Decorative Treatment

Base Shape/Curvature

Base Diameter/Height (cm)

Base Surface/Decorative Treatment

Appendages/Handles

Notes
8-7 / 1386 / 3CL23

bowl, carinated

K8279

Means/Cook Engraved variant?

engraved

Enos $10::$ plain

Compact, Hard, Smooth, Mica in paste

Grog + leached shell, fine and sparse

DARK GRAY (5YR4/1), REDDISH BROWN (5YR5/3)

VERY DARK GRAY (5YR3/1)

6.5

358

18.5

Encrustations; fireclouding; partial vessel, reconstructed with glue, missing about 1/4 of rim and sherd from body; 1386 inked on vessel

Bowl, Carinated

Burnished, flat, everted, sl. beveled in, 0.4; punctations

18.0

3.0

Inslanted, Carinated

Smoothed, engraved horizontal and vertical lines, ticked lines, trace white pigment?, punctations at lip and carination(sim. vessels Standridge, Lemley coll.)

$0.5-0.6$

Convex, continuous with base

18.5

3.5

Burnished; Plain

Undistinguished/Flat

7.5

Smoothed/Plain

No information; mis-identified as Phillips photo 3988 (Huddleston 118) 
JEC Hodges Collection, 77-1

Artifact No. / Old Cat. No. / Site No.

Vessel Form

ARAS/HSU Digital Photo

Type

Decoration

Rim :: Body

Paste

Temper

Core Color (Munsell)

Exterior Color (Munsell)

Interior Color (Munsell)

Vessel Overall Height $(\mathrm{cm})$

Vessel Overall Weight (g)

Vessel Maximum Diameter $(\mathrm{cm})$

Volume (liters)

Usewear/Sooting/Condition

Shape/Description

Lip Treatment/Shape/Angle/Thickness

Orifice Exterior Diameter $(\mathrm{cm})$

Rim/Neck Height (cm)

Neck Mid/Base Exterior Diameter (cm)

Rim/Neck Shape/Angle

Rim/Neck Surface/Decorative Treatment

Rim/Neck/Body Thickness (cm)

Body Shape

Body Maximum Diameter (cm)

Body Height $(\mathrm{cm})$

Body Surface/Decorative Treatment

Base Shape/Curvature

Base Diameter/Height (cm)

Base Surface/Decorative Treatment

Appendages/Handles

Notes
8-8 / 3492 / 3CL23

bottle

K5249

plain

Compact, Hard, Smooth

abundant fine leached shell + occasional grog

DARK GRAY (10YR4/1)

BLACK (10YR2/1), VERY PALE BROWN (10YR8/2)

23.5

903

19.2

2.2

base is roughened; some fireclouding; vessel is complete, small chip from lip

Bottle

Smoothed, rounded, everted, 0.4

4.3

6.8

$4.0 / 4.8$

vertical, slight spool

Smoothed

0.5

Biconical

19.2

16.2

Smoothed; Plain

circular/flat

6.3

Smoothed/Plain

Huddleston ('found 2 pcs'), 12/7/1940

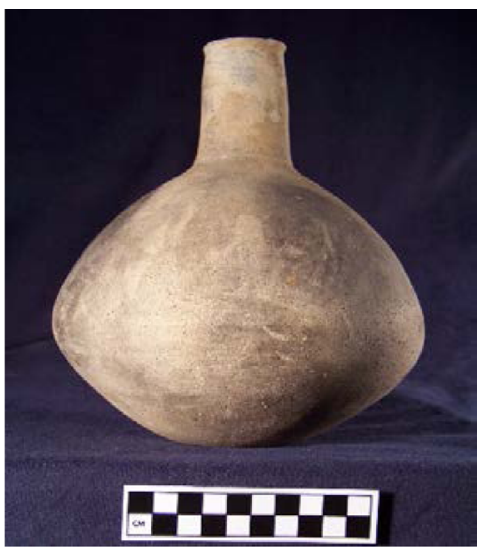




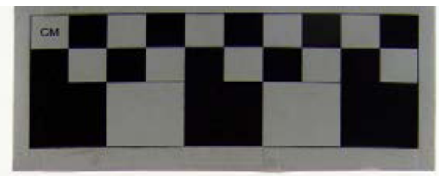

Artifact No. / Old Cat. No. / Site No.

Vessel Form

ARAS/HSU Digital Photo

Type

Decoration

Rim :: Body

Paste

Temper

Core Color (Munsell)

Exterior Color (Munsell)

Interior Color (Munsell)

Vessel Overall Height (cm)

Vessel Overall Weight (g)

Vessel Maximum Diameter $(\mathrm{cm})$

Volume (liters)

Usewear/Sooting/Condition

Shape/Description

Lip Treatment/Shape/Angle/Thickness Orifice Exterior Diameter (cm)

Rim/Neck Height (cm)

Neck Mid/Base Exterior Diameter (cm)

Rim/Neck Shape/Angle

Rim/Neck Surface/Decorative Treatment

Rim/Neck/Body Thickness (cm)

Body Shape

Body Maximum Diameter (cm)

Body Height $(\mathrm{cm})$

Body Surface/Decorative Treatment

Base Shape/Curvature

Base Diameter/Height (cm)

Base Surface/Decorative Treatment

Appendages/Handles

Notes
8-9 / 1219/3CL23

bowl, simple

K8318

Hempstead Engraved?

engraved

Erie $11::$ Elmira 12

Compact, Hard, Smooth

Grog, medium and abundant + shell (leached, voids), medium, abundant

\section{VERY DARK GRAY (7.5YR3/1), PINK (7.5YR8/4), REDDISH YELLOW (5YR6/6)}

\section{PINKISH GRAY (5YR6/2)}

7.5

382

15.2

0.8

Erosion (post-depositional); fireclouding; significant amount of soil inside pot; vessel is complete; 1219 inked on base

Bowl, simple

eroded, rounded, none, 0.4

15.2

4.6

convex, inslanted

eroded, engraved, 2 horizontal lines 4-6mm apart (a third is top of body decoration)

$0.5-0.6$

Convex

15.2

3.2

Eroded; Engraved, similar to Elmira 12 but wavy not pointed lines on bottom of design; crosshatched area, undulating line

Undistinguished/Convex

eroded/plain

Huddleston Grave 9, 1/21/1939; Phillips photo 3853 (Huddleston 126); early Mid-Ouachita phase 
Artifact No. / Old Cat. No. / Site No.

Vessel Form

ARAS/HSU Digital Photo

Type

Decoration

Rim :: Body

Paste

Temper

Core Color (Munsell)

Exterior Color (Munsell)

Interior Color (Munsell)

Vessel Overall Height $(\mathrm{cm})$

Vessel Overall Weight (g)

Vessel Maximum Diameter $(\mathrm{cm})$

Volume (liters)

Usewear/Sooting/Condition

Shape/Description

Lip Treatment/Shape/Angle/Thickness

Orifice Exterior Diameter $(\mathrm{cm})$

Rim/Neck Height (cm)

Neck Mid/Base Exterior Diameter (cm)

Rim/Neck Shape/Angle

Rim/Neck Surface/Decorative Treatment

Rim/Neck/Body Thickness (cm)

Body Shape

Body Maximum Diameter (cm)

Body Height (cm)

Body Surface/Decorative Treatment

Base Shape/Curvature

Base Diameter/Height (cm)

Base Surface/Decorative Treatment

Appendages/Handles

Notes
8-10/1225 / 3CL23

seed jar

K8376

plain

Compact, Hard, Smooth, Mica in paste

grog, medium \& abundant + shell, medium \& abundand, leached

REDDISH YELLOW (5YR6/8)

DK YELLOW BRN (10YR4/4), DARK BROWN (7.5YR3/4), BROWN (7.5YR4/4)

19.7

956

15.4

2.1

Very eroded; fireclouding; partial vessel, reconstructed with glue, sherd missing from body, 2 probe holes in body; 1225 inked near base

Seed Jar

Smoothed, rounded, none

3.3

Convex, Inslanted

Smoothed (eroded); Plain

Ovoid

15.4

19.7

Smoothed (eroded); Plain

circular/flat

8.5

Smoothed/Plain

Huddleston Grave 7, 1/14/1938[1939]; Phillips photo 3935 (Huddleston 114), Mid-Ouachita phase 
Artifact No. / Old Cat. No. / Site No.

Vessel Form

ARAS/HSU Digital Photo

Type

Decoration

Rim :: Body

Paste

Temper

Core Color (Munsell)

Exterior Color (Munsell)

Interior Color (Munsell)

Vessel Overall Height $(\mathrm{cm})$

Vessel Overall Weight (g)

Vessel Maximum Diameter (cm)

Volume (liters)

Usewear/Sooting/Condition

Shape/Description

Lip Treatment/Shape/Angle/Thickness

Orifice Exterior Diameter $(\mathrm{cm})$

Rim/Neck Height (cm)

Neck Mid/Base Exterior Diameter $(\mathrm{cm})$

Rim/Neck Shape/Angle

Rim/Neck Surface/Decorative Treatment

Rim/Neck/Body Thickness (cm)

Body Shape

Body Maximum Diameter $(\mathrm{cm})$

Body Height (cm)

Body Surface/Decorative Treatment

Base Shape/Curvature

Base Diameter/Height $(\mathrm{cm})$

Base Surface/Decorative Treatment

Appendages/Handles

Notes
8-11 / 1203 / 3CL23

bowl, carinated

K8422

Friendship Engraved var. Freeman

engraved

Elaine 1 :: plain

Compact, Hard, Smooth, Mica in paste

grog, fine, abundant + leached shell, fine, sparse

GRAY (7.5YR5/1)

BLACK (7.5YR2.5/1), BROWN (7.5YR5/2-5/4)

BLACK (7.5YR2.5/1), BROWN (7.5YR5/4)

9.8

797

22.2

2.1

Some wear around lip and roughening on exterior base (use?); fireclouding; poss. probe hole at lip; 1203 inked on base, remnant of paper tag inside

Bowl, Carinated

Burnished, rounded/thinned, sl. everted, 0.3

21.1

5.0

straight, inslanted

Burnished, engraved, 3 tier decoration of scrolls with squared ends and ticked line rectangles within, crosshatched in between, repeats $4 \mathrm{x}$

0.6

Convex

22.2

4.8

Burnished; Plain

Undistinguished/Convex

Burnished/Plain 
Artifact No. / Old Cat. No. / Site No.

Vessel Form

ARAS/HSU Digital Photo

Type

Decoration

Rim :: Body

Paste

Temper

Core Color (Munsell)

Exterior Color (Munsell)

Interior Color (Munsell)

Vessel Overall Height (cm)

Vessel Overall Weight (g)

Vessel Maximum Diameter $(\mathrm{cm})$

Volume (liters)

Usewear/Sooting/Condition

Shape/Description

Lip Treatment/Shape/Angle/Thickness

Orifice Exterior Diameter $(\mathrm{cm})$

Rim/Neck Height (cm)

Neck Mid/Base Exterior Diameter $(\mathrm{cm})$

Rim/Neck Shape/Angle

Rim/Neck Surface/Decorative Treatment

Rim/Neck/Body Thickness (cm)

Body Shape

Body Maximum Diameter $(\mathrm{cm})$

Body Height $(\mathrm{cm})$

Body Surface/Decorative Treatment

Base Shape/Curvature

Base Diameter/Height $(\mathrm{cm})$

Base Surface/Decorative Treatment

Appendages/Handles

Notes
8-12/1221 / 3CL23

jar, tall rim

K8430

Military Road Incised

incised \& punctated

Bethel 27 :: Bard 1

Crumbly, Soft, Smooth

leached shell, coarse and abundant

DARK GRAY (10YR4/1)

BROWN (10YR4/3), VERY DARK BROWN (10YR2/2), PALE BROWN (10YR6/3)

LIGHT BROWNISH GRAY (10YR6/2), BLACK (10YR2/1), DARK GRAY (10YR4/1)

15.8

432

17.7

Eroded; trace sooting on interior rim; missing most of base and a few body and rim sherds; 1221 inked on interior

Jar, Tall Rim

smoothed, flattened/beveled out, sl. everted, 0.3

17.7

6.0

Straight, Outslanted

Smoothed; incised \& punctated; meandering horizontal trailed lines between two rows of vertical punctations

$0.6-0.5$

Low Waist

16.0

9.8

Smoothed; incised \& punctated; horizontal and meandering trailing alternating with meandering lines of vertical punctations

circular/flat

8.8

Eroded/Plain, mostly missing

Huddleston, Rorie Mound; Phillips photo 3898 (Huddleston 219) 
Artifact No. / Old Cat. No. / Site No.

Vessel Form

ARAS/HSU Digital Photo

Type

Decoration

Rim :: Body

Paste

Temper

Core Color (Munsell)

Exterior Color (Munsell)

Interior Color (Munsell)

Vessel Overall Height $(\mathrm{cm})$

Vessel Overall Weight (g)

Vessel Maximum Diameter $(\mathrm{cm})$

Volume (liters)

Usewear/Sooting/Condition

Shape/Description

Lip Treatment/Shape/Angle/Thickness

Orifice Exterior Diameter $(\mathrm{cm})$

Rim/Neck Height (cm)

Neck Mid/Base Exterior Diameter (cm)

Rim/Neck Shape/Angle

Rim/Neck Surface/Decorative Treatment

Rim/Neck/Body Thickness (cm)

Body Shape

Body Maximum Diameter (cm)

Body Height (cm)

Body Surface/Decorative Treatment

Base Shape/Curvature

Base Diameter/Height (cm)

Base Surface/Decorative Treatment

Appendages/Handles

Notes
8-13 / 1224 / 3CL23

beaker

K8457

Hempstead Engraved

engraved

Erie 13 :: Eccles 1

Compact, Hard, Smooth

shell mostly leached, medium/abundant + grog, fine, sparse

V DK GRAY BRN (10YR3/2), BLACK (10YR2/1), LT BROWNISH GRAY (10YR6/2)

BLACK (10YR2/1), LIGHT BROWNISH GRAY (10YR6/2), GRAYISH BROWN (10YR5/2)

11.9

343

11.8

0.8

Partial vessel, reconstructed in past with gray plaster in past (bag of gray clay inside vessel may be Hodges plaster), several sherds missing from rim/body; 1224 inked on base

Beaker or carinated bowl with rolled lip

burnished, rounded, none, 0.4

11.8

4.4

straight, vertical

Burnished, engraved, 6 horizontal lines, fine engraving 5-8 $\mathrm{mm}$ apart

0.5

Low Waist

11.2

7.5

Burnished; Engraved, 13 crosshatched pendant triangles from engraved horizontal line at top of body

Undistinguished/Convex

Burnished/Plain

2 lugs set at rim/lip, plain, each is perforated vertically

Huddleston, Rorie Mound; Phillips photo 3859 ("deep bowl, perforated lugs"; Huddleston 214) 
JEC Hodges Collection, 77-1

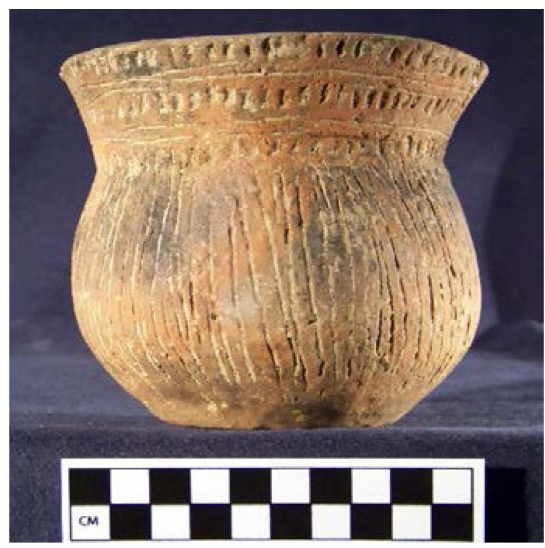

Artifact No. / Old Cat. No. / Site No.

Vessel Form

ARAS/HSU Digital Photo

Type

Decoration

Rim :: Body

Paste

Temper

Core Color (Munsell)

Exterior Color (Munsell)

Interior Color (Munsell)

Vessel Overall Height $(\mathrm{cm})$

Vessel Overall Weight (g)

Vessel Maximum Diameter $(\mathrm{cm})$

Volume (liters)

Usewear/Sooting/Condition

Shape/Description

Lip Treatment/Shape/Angle/Thickness

Orifice Exterior Diameter $(\mathrm{cm})$

Rim/Neck Height $(\mathrm{cm})$

Neck Mid/Base Exterior Diameter $(\mathrm{cm})$

Rim/Neck Shape/Angle

Rim/Neck Surface/Decorative Treatment

Rim/Neck/Body Thickness (cm)

Body Shape

Body Maximum Diameter $(\mathrm{cm})$

Body Height (cm)

Body Surface/Decorative Treatment

Base Shape/Curvature

Base Diameter/Height $(\mathrm{cm})$

Base Surface/Decorative Treatment

Appendages/Handles

Notes
8-14 / 1228 / 3CL23

jar, short rim

K5301

Military Road Incised variant?

incised \& punctated

Beloit 6 :: Abraham 6

Compact, Hard, Smooth

Grog, abundant and coarse

REDDISH BROWN (5YR5/4), REDDISH GRAY (5YR4/2)

DARK GRAY (5YR4/1)

11.5

457

12.0

0.6

Sooting at neck on exterior and inside rim/neck; partial vessel reconstructed in past, missing/unattached pcs in bag

Jar, Short Rim

Smoothed, rounded, 0.5

12.0

3.0

straight, outslanted

Smoothed, Incised \& Punctations, rows of vertical tool punctates alternate with 2 horizontal lines 0.5

Subglobular

11.0

8.5

Smoothed; Incised, wet paste, vertical incised lines from below rim to $1.5 \mathrm{~cm}$ above base of vessel; similar body treatment to Pease B-I from East site

circular/flat

6.5

Smoothed/Plain

Huddleston Grave 9, 1/21/1939; Phillips photo 3872 (Huddleston 128); early Mid-Ouachita phase 


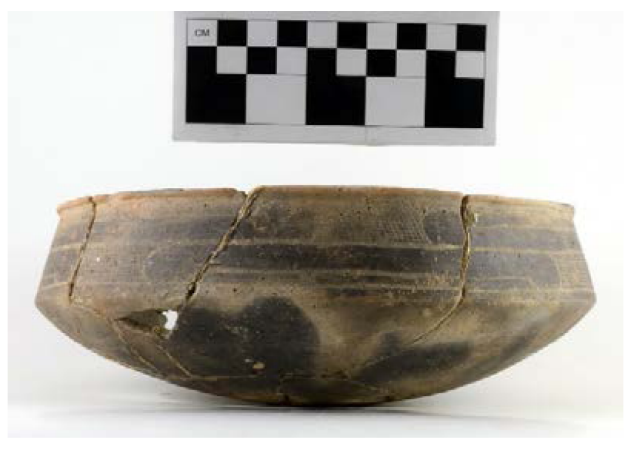

Artifact No. / Old Cat. No. / Site No.

Vessel Form

ARAS/HSU Digital Photo

Type

Decoration

Rim :: Body

Paste

Temper

Core Color (Munsell)

Exterior Color (Munsell)

Interior Color (Munsell)

Vessel Overall Height $(\mathrm{cm})$

Vessel Overall Weight (g)

Vessel Maximum Diameter $(\mathrm{cm})$

Volume (liters)

Usewear/Sooting/Condition

Shape/Description

Lip Treatment/Shape/Angle/Thickness

Orifice Exterior Diameter $(\mathrm{cm})$

Rim/Neck Height (cm)

Neck Mid/Base Exterior Diameter $(\mathrm{cm})$

Rim/Neck Shape/Angle

Rim/Neck Surface/Decorative Treatment

Rim/Neck/Body Thickness (cm)

Body Shape

Body Maximum Diameter $(\mathrm{cm})$

Body Height (cm)

Body Surface/Decorative Treatment

Base Shape/Curvature

Base Diameter/Height (cm)

Base Surface/Decorative Treatment

Appendages/Handles
8-15 / 1227 / 3CL23

bowl, carinated

N3037

Friendship Engraved var. Freeman

engraved

Elaine $20 ? 40 ?::$ plain

Compact, Hard, Smooth

Shell (leached), medium \& adundant + grog, medium \& sparse

DARK GRAY (10YR4/1)

GRAY (7.5YR6/1, 10YR5/1), LIGHT REDDISH BROWN (5YR6/4)

GRAY (10YR5/1), WHITE (10YR8/1), DARK GRAY (7.5YR4/1)

9.0

579

22.5

1.9

Fireclouding; partial vessel, reconstructed in past, missing several pcs from body, lip chipping; 1227 inked on base, paper tag inside

Bowl, Carinated

Burnished, beveled, 0.4

22.5

4.0

straight, inslanted

Bunished, Engraved, 2 panels, engraved scrolls with hatching, cross hatching between

$0.5-0.6$

Convex

22.5

5.0

Burnished; Plain

UndistinguishedConvex

Smoothed/Plain

Huddleston Grave 7, 1/14/1938[1939]; Phillips photo 3958 (Huddleston 115), Mid-Ouachita phase 
JEC Hodges Collection, 77-1

Artifact No. / Old Cat. No. / Site No.

Vessel Form

ARAS/HSU Digital Photo

Type

Decoration

Rim :: Body

Paste

Temper

Core Color (Munsell)

Exterior Color (Munsell)

Interior Color (Munsell)

Vessel Overall Height (cm)

Vessel Overall Weight (g)

Vessel Maximum Diameter $(\mathrm{cm})$

Volume (liters)

Usewear/Sooting/Condition

Shape/Description

Lip Treatment/Shape/Angle/Thickness

Orifice Exterior Diameter (cm)

Rim/Neck Height (cm)

Neck Mid/Base Exterior Diameter $(\mathrm{cm})$

Rim/Neck Shape/Angle

Rim/Neck Surface/Decorative Treatment

Rim/Neck/Body Thickness (cm)

Body Shape

Body Maximum Diameter (cm)

Body Height (cm)

Body Surface/Decorative Treatment

Base Shape/Curvature

Base Diameter/Height $(\mathrm{cm})$

Base Surface/Decorative Treatment

Appendages/Handles

Notes
$8-16 / 1223$ / 3CL23

jar, tall rim

N3065

Military Road Incised

incised \& punctated

Bethel 1 :: Bard 14

Compact, Hard, Smooth

Grog, medium \& abundant

LIGHT GRAY (10YR7/1)

BROWN (7.5YR5/4), LIGHT GRAY (10YR7/2), BLACK (7.5YR2.5/1)

BROWN (7.5YR5/4), LIGHT GRAY (10YR7/1-7/2)

20.9

1473

20.9

Some sooting interior rim/body and exterior rim/body; vessel reconstructed in past, reglued, sev. sherds missing from base; 1223 inked inside rim

Jar, Tall Rim

smoothed, beveled out, sl. everted, 0.4

20.9

7.1

straight, outslanted

Smoothed, Incised \& Punctated, double row vertical punctations and meandering incising

0.6

Subglobular

19.8

13.8

Smoothed; Incised \& Punctated; double row of vertical punctations, meandering, and horizontal meandering incising

circular/flat

9.0

Smoothed/Plain

Huddleston Grave 8, 1/14/1938[1939]; Phillips photo 3987 (Huddleston 120); early Mid-Ouachita phase 
Artifact No. / Old Cat. No. / Site No.

Vessel Form

ARAS/HSU Digital Photo

Type

Decoration

$\operatorname{Rim}::$ Body

Paste

Temper

Core Color (Munsell)

Exterior Color (Munsell)

Interior Color (Munsell)

Vessel Overall Height $(\mathrm{cm})$

Vessel Overall Weight (g)

Vessel Maximum Diameter (cm)

Volume (liters)

Usewear/Sooting/Condition

Shape/Description

Lip Treatment/Shape/Angle/Thickness

Orifice Exterior Diameter (cm)

Rim/Neck Height (cm)

Neck Mid/Base Exterior Diameter (cm)

Rim/Neck Shape/Angle

Rim/Neck Surface/Decorative Treatment

Rim/Neck/Body Thickness (cm)

Body Shape

Body Maximum Diameter $(\mathrm{cm})$

Body Height (cm)

Body Surface/Decorative Treatment

Base Shape/Curvature

Base Diameter/Height $(\mathrm{cm})$

Base Surface/Decorative Treatment

Appendages/Handles
8-17 / 1389 / 3CL23

bowl, simple

N3134

engraved/excised

Cornell 9, Elgin 1 :: plain

Compact, Hard, Smooth

Grog, fine \& sparse

\section{STRONG BROWN (7.5YR5/6), VERY DARK GRAY (2.5Y3/1)}

BROWN (10YR5/3), DARK GRAY (2.5Y4/1)

7.8

548

22.7

1.2

Roughening on base; fireclouding on int and ext; vessel reconstructed in past, several sm pcs from body and base; 1389 inked on side, remnants of paper sticker on interiror

Bowl, simple

everted, 0.6; cogged edge, engraved and excised triangles on top of lip

22.7

continuous with body

Smoothed, plain (cogged rim, decoration limited to top of flange)

0.4

convex

22.7

7.8

Smoothed; Plain

Undistinguished/Convex

Smoothed/Plain

Huddleston Grave 9, 1/21/1939; Phillips photo 3954 (Huddleston 129); early Mid-Ouachita

Notes 


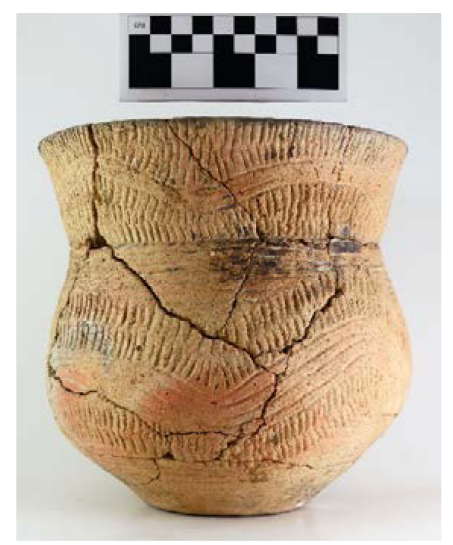

Artifact No. / Old Cat. No. / Site No.

Vessel Form

ARAS/HSU Digital Photo

Type

Decoration

Rim :: Body

Paste

Temper

Core Color (Munsell)

Exterior Color (Munsell)

Interior Color (Munsell)

Vessel Overall Height $(\mathrm{cm})$

Vessel Overall Weight (g)

Vessel Maximum Diameter (cm)

Volume (liters)

Usewear/Sooting/Condition

Shape/Description

Lip Treatment/Shape/Angle/Thickness

Orifice Exterior Diameter $(\mathrm{cm})$

Rim/Neck Height (cm)

Neck Mid/Base Exterior Diameter $(\mathrm{cm})$

Rim/Neck Shape/Angle

Rim/Neck Surface/Decorative Treatment

Rim/Neck/Body Thickness (cm)

Body Shape

Body Maximum Diameter (cm)

Body Height (cm)

Body Surface/Decorative Treatment

Base Shape/Curvature

Base Diameter/Height (cm)

Base Surface/Decorative Treatment

Appendages/Handles

Notes
8-18 / 2101 / 3CL23

jar, tall rim

N2502

Military Road Incised

incised \& punctated

Bethel 1 :: Bard 14

Compact, Hard, Smooth

grog, medium \& abundant, occasional voids

VERY PALE BROWN (10YR8/2), REDDISH YELLOW (5YR6/6), PINK (5YR7/4)

VERY PALE BROWN (10YR7/3), DARK GRAY (10YR4/1)

22.0

1807

20.3

Base is roughened; soot on exterior, fireclouding; vessel reconstructed in past, missing 2-3 sm sherds; 2101 inked at base

Jar, Tall Rim

Smoothed, flat, none, 0.4

19.8

6.0

Concave, outslanted

Smoothed, trailed/incised, meandering horizontal lines between rows of vertical slash punctations, tool?

$0.4-1.0$

Low Waist

20.3

16.0

Smoothed; Incised/Trailed, horizontal lines alternate with meandering rows of vertical punctations and meandering lines

circular/flat

9.0

Smoothed/Plain

Huddleston Grave 13, 1/1/1940; early Mid-Ouachita phase 
Artifact No. / Old Cat. No. / Site No.

Vessel Form

ARAS/HSU Digital Photo

Type

Decoration

Rim :: Body

Paste

Temper

Core Color (Munsell)

Exterior Color (Munsell)

Interior Color (Munsell)

Vessel Overall Height $(\mathrm{cm})$

Vessel Overall Weight (g)

Vessel Maximum Diameter (cm)

Volume (liters)

Usewear/Sooting/Condition

Shape/Description

Lip Treatment/Shape/Angle/Thickness

Orifice Exterior Diameter $(\mathrm{cm})$

Rim/Neck Height (cm)

Neck Mid/Base Exterior Diameter $(\mathrm{cm})$

Rim/Neck Shape/Angle

Rim/Neck Surface/Decorative Treatment

Rim/Neck/Body Thickness (cm)

Body Shape

Body Maximum Diameter (cm)

Body Height (cm)

Body Surface/Decorative Treatment

Base Shape/Curvature

Base Diameter/Height (cm)

Base Surface/Decorative Treatment

Appendages/Handles

Notes
$8-19 / 1208$ / 3CL23

seed jar

N2793

plain

Compact, Hard, Smooth

Shell (leached), medium \& abundant + grog, medium \& sparse

LIGHT REDDISH BROWN (7.5YR6/4), DARK GRAY (7.5YR4/1), WHITE (2.5Y8/1)

19.5

872

14.5

1.8

Fireclouding; vessel intact except for cracks in body; 1208 inked on lower body

Seed Jar

smoothed, rounded, none, 0.6

3.4

continuous with body

Smoothed, plain

barrel

14.5

17.5

Smoothed; Plain

circular/slightly convex

12.5

Smoothed/Plain

Huddleston Grave 11, 2/11/1939; Phillips photo 3934 (Huddleston 151 [prob. 150]); Mid-Ouachita phase 
Artifact No. / Old Cat. No. / Site No.

Vessel Form

ARAS/HSU Digital Photo

Type

Decoration

Rim :: Body

Paste

Temper

Core Color (Munsell)

Exterior Color (Munsell)

Interior Color (Munsell)

Vessel Overall Height $(\mathrm{cm})$

Vessel Overall Weight (g)

Vessel Maximum Diameter $(\mathrm{cm})$

Volume (liters)

Usewear/Sooting/Condition

Shape/Description

Lip Treatment/Shape/Angle/Thickness

Orifice Exterior Diameter $(\mathrm{cm})$

Rim/Neck Height (cm)

Neck Mid/Base Exterior Diameter $(\mathrm{cm})$

Rim/Neck Shape/Angle

Rim/Neck Surface/Decorative Treatment

Rim/Neck/Body Thickness (cm)

Body Shape

Body Maximum Diameter (cm)

Body Height $(\mathrm{cm})$

Body Surface/Decorative Treatment

Base Shape/Curvature

Base Diameter/Height (cm)

Base Surface/Decorative Treatment

Appendages/Handles

Notes
8-20 / 2099 / 3CL23

seed jar, fragment

N2767

plain

Compact, Soft, Gritty

Grog, medium \& abundant

REDDISH YELLOW (5YR6/6), WHITE (7.5YR8/1), GRAY (7.5YR5/1)

REDDISH YELLOW (7.5YR6/6), GRAY (7.5YR6/1), VERY DARK GRAY (10YR3/1)

YELLOW (10YR7/6), GRAY (7.5YR5/1)

13.5

453

14.5

Fireclouding; partial vessel, missing upper half; 2099 inked on side

Probable seed jar

missing

0.6

Ovoid

14.5

13.5 Partial

Burnished; Plain

Circular/Flat

8.0

Smoothed/Plain

Huddleston Grave 13, 1/1/1940; early Mid-Ouachita phase 


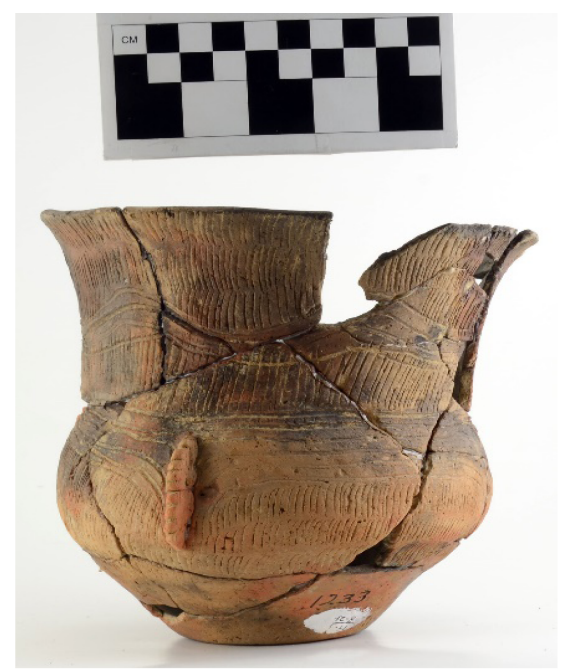

Artifact No. / Old Cat. No. / Site No.

Vessel Form

ARAS/HSU Digital Photo

Type

Decoration

Rim :: Body

Paste

Temper

Core Color (Munsell)

Exterior Color (Munsell)

Interior Color (Munsell)

Vessel Overall Height $(\mathrm{cm})$

Vessel Overall Weight (g)

Vessel Maximum Diameter $(\mathrm{cm})$

Volume (liters)

Usewear/Sooting/Condition

Shape/Description

Lip Treatment/Shape/Angle/Thickness

Orifice Exterior Diameter (cm)

Rim/Neck Height (cm)

Neck Mid/Base Exterior Diameter (cm)

Rim/Neck Shape/Angle

Rim/Neck Surface/Decorative Treatment

Rim/Neck/Body Thickness (cm)

Body Shape

Body Maximum Diameter (cm)

Body Height (cm)

Body Surface/Decorative Treatment

Base Shape/Curvature

Base Diameter/Height $(\mathrm{cm})$

Base Surface/Decorative Treatment

Appendages/Handles

Notes
8-21 / 1233 / 3CL23

jar, tall rim

N3264

Military Road Incised variant?

incised/punctated/applique

Bethel 1 :: Bard 10, 40 (sim.)

Compact, Soft, Smooth, Mica in paste

leached shell, medium, medium

DARK BROWN (7.5YR3/2)

DK BRN (7.5YR3/2), LT BRN (7.5YR6/4), STRONG BROWN (7.5YR5/6)

BLACK (7.5YR2.5/1), VERY DARK GRAY (7.5YR3/1)

16.0

401

16.0

Sooting on rim interior and exterior, some fireclouding; partial vessel, reconstructed in past, 1/2 of rim and some body sherds missing; 1233 inked on lower body

Jar

Smoothed, flat, sl. everted, 0.4

16.0

7.0

Concave, outslanted

Smoothed, Incised/punctations, 2-3 rows of sharp vertical punctations over 4 meandering incised lines over 1 row long vertical punctations

$0.4-0.6$

Subglobular

14.5

9.0

Smoothed; Incised \& Punctated, 6 rows irregular incising, dipping below appique strips, 3 rows of shert vertical punctations below; plain lower $3.5 \mathrm{~cm}$ of body; Military Road dec. with vertical appliques sim. to Pease B-I or Haley C-I

Circular/Flat

6.5

Smoothed/Plain

2 (originally 4) short applique ridges with notching on shoulders

Huddleston, Rorie Mound; Phillips photo 3899 (Huddleston 212) 


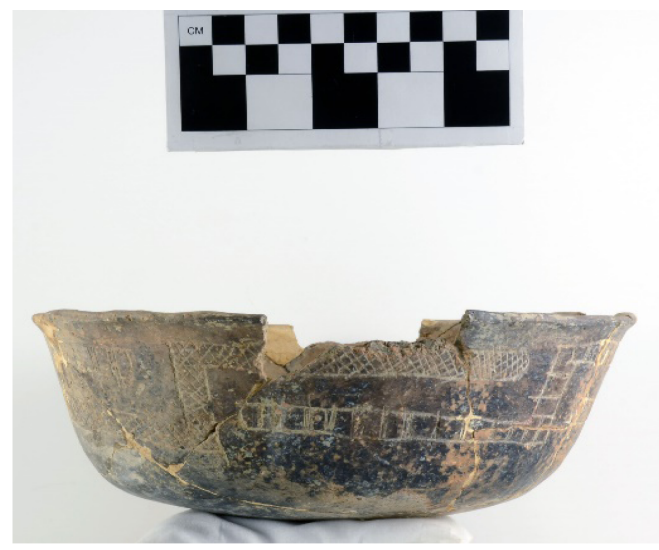

Artifact No. / Old Cat. No. / Site No.

Vessel Form

ARAS/HSU Digital Photo

Type

Decoration

Rim :: Body

Paste

Temper

Core Color (Munsell)

Exterior Color (Munsell)

Interior Color (Munsell)

Vessel Overall Height $(\mathrm{cm})$

Vessel Overall Weight (g)

Vessel Maximum Diameter (cm)

Volume (liters)

Usewear/Sooting/Condition

Shape/Description

Lip Treatment/Shape/Angle/Thickness

Orifice Exterior Diameter $(\mathrm{cm})$

Rim/Neck Height (cm)

Neck Mid/Base Exterior Diameter $(\mathrm{cm})$

Rim/Neck Shape/Angle

Rim/Neck Surface/Decorative Treatment

Rim/Neck/Body Thickness (cm)

Body Shape

Body Maximum Diameter (cm)

Body Height $(\mathrm{cm})$

Body Surface/Decorative Treatment

Base Shape/Curvature

Base Diameter/Height $(\mathrm{cm})$

Base Surface/Decorative Treatment

Appendages/Handles

Notes
$8-22$ / 1220 / 3CL23

bowl, simple

N3437

Friendship Engraved var. Meador

engraved

Elwyn 15 (sim.) :: plain

Compact, Hard, Smooth

Grog, coarse \& abundant

DARK GRAY (7.5YR4/1)

PINK (7.5YR7/4-8/3), BLACK (7.5YR2.5/1)

DARK GRAY (7.5YR4/1), LIGHT BROWN (7.5YR6/3), BLACK (7.5YR2.5/1)

8.0

504

20.5

1.3

Partial vessel, reconstructed with plaster in past, missing pcs from rim, body; 1220 inked on base, remnants of paper tag inside bowl

Bowl, simple

Burnished, rounded, everted, 0.5

20.5

1.0

Concave, outslanted

Burnished, Engraved, geometric pattern, alternating crosshatching and ladders

0.5

Convex

19.8

7.0

Burnished; Plain

Undistinguished/Slightly convex

Burnished/Plain

Huddleston Grave 4, 3/19/1938; Phillips photo 3955 (Huddleston 40), early Mid-Ouachita phase 
JEC Hodges Collection, 77-1

Artifact No. / Old Cat. No. / Site No.

Vessel Form

ARAS/HSU Digital Photo

Type

Decoration

Rim :: Body

Paste

Temper

Core Color (Munsell)

Exterior Color (Munsell)

Interior Color (Munsell)

Vessel Overall Height $(\mathrm{cm})$

Vessel Overall Weight (g)

Vessel Maximum Diameter $(\mathrm{cm})$

Volume (liters)

Usewear/Sooting/Condition

Shape/Description

Lip Treatment/Shape/Angle/Thickness

Orifice Exterior Diameter $(\mathrm{cm})$

Rim/Neck Height $(\mathrm{cm})$

Neck Mid/Base Exterior Diameter (cm)

Rim/Neck Shape/Angle

Rim/Neck Surface/Decorative Treatment

Rim/Neck/Body Thickness (cm)

Body Shape

Body Maximum Diameter $(\mathrm{cm})$

Body Height (cm)

Body Surface/Decorative Treatment

Base Shape/Curvature

Base Diameter/Height (cm)

Base Surface/Decorative Treatment

Appendages/Handles

Notes
8-23 / 1229 / 3CL23

jar, tall rim

N3584

Military Road Incised

incised \& punctated

Bethel 1 :: Bard 15

Compact, Hard, Smooth

Grog, coarse, abundant; a few voids

PINKISH GRAY (7.5YR6/2)

REDDISH YELLOW (7.5YR6/6), BROWN (7.5YR4/2), GRAY (7.5YR5/1)

BLACK (7.5YR2.5/1), LIGHT BROWN (7.5YR6/3)

18.8

1018

18.9

Sooting on rim/upper body interior and exterior, fireclouding; partial vessel, reconstructed in past and reglued, missing 1/3 of body; 1229 inked on interior of one sherd

Jar, Tall Rim

smoothed, flat, everted, 0.4

18.9

5.7

straight, outslanted

Smoothed, Punctated \& incised/trailed, 2-3 rows of punctations, 4 meandering trailed horizontal lines, 2-3 rows of punctations

0.7

Subglobular

18.4

13.1

Smoothed; Punctated \& Incised/Trailed, horizontal trailed lines, 2 rows of punctates meandering, horizontal lines, 2 rows punctations at base

Circular/Flat

10.0

Smoothed/Plain

Huddleston Grave 4, 3/19/1938; Phillips photo 3938 (Huddleston 41), early Mid-Ouachita phase 
Artifact No. / Old Cat. No. / Site No.

Vessel Form

ARAS/HSU Digital Photo

Type

Decoration

Rim :: Body

Paste

Temper

Core Color (Munsell)

Exterior Color (Munsell)

Interior Color (Munsell)

Vessel Overall Height (cm)

Vessel Overall Weight (g)

Vessel Maximum Diameter (cm)

Volume (liters)

Usewear/Sooting/Condition

Shape/Description

Lip Treatment/Shape/Angle/Thickness

Orifice Exterior Diameter $(\mathrm{cm})$

Rim/Neck Height (cm)

Neck Mid/Base Exterior Diameter $(\mathrm{cm})$

Rim/Neck Shape/Angle

Rim/Neck Surface/Decorative Treatment

Rim/Neck/Body Thickness (cm)

Body Shape

Body Maximum Diameter $(\mathrm{cm})$

Body Height (cm)

Body Surface/Decorative Treatment

Base Shape/Curvature

Base Diameter/Height $(\mathrm{cm})$

Base Surface/Decorative Treatment

Appendages/Handles

Notes
8-24 / 2100 / 3CL23

jar, tall rim

N3713

Sinner Linear Punctated

punctations

Cambridge 6 (type) :: Carver 30 (type)

Compact, Hard, Smooth

Grog, abundant \& medium

VERY DARK GRAY (5YR3/1), LIGHT REDDISH BROWN (5YR6/4)

VERY DARK GRAY (5YR3/1)

14.2

481

13.0

Sooting on interior rim, fireclouding; partial vessel, reconstructed in past, missing 4 sm sherds; 2100 inked near base

Jar, Tall Rim

Smoothed, rounded, sl. everted, 0.3

13.0

7.2

Concave, outslanted

Smoothed, Linear punctations, 10 horizontal lines, vertical fine lines, scalloped single line, more vertical fine lines all of punctations

$0.4-0.5$

high waisted

12.2

7.0

Smoothed; Linear punctations, 6 horizontal lines at junction of rim/body, 2 rows of punctated basket weave; design ends $1.5 \mathrm{~cm}$ above base

Circular/Flat

6.5

Smoothed/Plain

Huddleston Grave 13, 1/1/1940; early Mid-Ouachita phase 
Artifact No. / Old Cat. No. / Site No.

Vessel Form

ARAS/HSU Digital Photo

Type

Decoration

Rim :: Body

Paste

Temper

Core Color (Munsell)

Exterior Color (Munsell)

Interior Color (Munsell)

Vessel Overall Height $(\mathrm{cm})$

Vessel Overall Weight (g)

Vessel Maximum Diameter (cm)

Volume (liters)

Usewear/Sooting/Condition

Shape/Description

Lip Treatment/Shape/Angle/Thickness

Orifice Exterior Diameter $(\mathrm{cm})$

Rim/Neck Height $(\mathrm{cm})$

Neck Mid/Base Exterior Diameter $(\mathrm{cm})$

Rim/Neck Shape/Angle

Rim/Neck Surface/Decorative Treatment

Rim/Neck/Body Thickness (cm)

Body Shape

Body Maximum Diameter (cm)

Body Height (cm)

Body Surface/Decorative Treatment

Base Shape/Curvature

Base Diameter/Height (cm)

Base Surface/Decorative Treatment

Appendages/Handles

Notes
8-25 / 3CL23

bowl, carinated

N3052

Friendship Engraved var. Meador

engraved

Elwyn $10::$ plain

Compact, Hard, Smooth

grog, abundant \& medium + shell, leached, sparse \& medium

REDDISH BROWN (2.5YR5/4-5YR5/3), DARK GRAY (5YR4/1)

VERY DARK GRAY (5YR3/1), LIGHT REDDISH BROWN (5YR6/4)

9.0 (estimated)

400 (partial)

23.0 (estimated)

Fireclouding; partial vessel/sherd lot, reconstructed in past and reglued

Bowl, Carinated

burnished, rounded, everted, 0.4

23.0 (estimated, $30 \%$ )

4.0

straight, carinated

Burnished, engraved, crosshatching, ovals, ladders

0.4

Convex

Burnished; plain

Undistinguished/Convex

burnished/plain

Huddleston Grave 3, 12/22-30/1938[1937?], (paper tag with pen/pencil "6-3-2 grave No 3 with frog 39"); Mid-Ouachita phase 
Artifact No. / Old Cat. No. / Site No.

Vessel Form

ARAS/HSU Digital Photo

Type

Decoration

Rim :: Body

Paste

Temper

Core Color (Munsell)

Exterior Color (Munsell)

Interior Color (Munsell)

Vessel Overall Height $(\mathrm{cm})$

Vessel Overall Weight (g)

Vessel Maximum Diameter (cm)

Volume (liters)

Usewear/Sooting/Condition

Shape/Description

Lip Treatment/Shape/Angle/Thickness

Orifice Exterior Diameter $(\mathrm{cm})$

Rim/Neck Height (cm)

Neck Mid/Base Exterior Diameter (cm)

Rim/Neck Shape/Angle

Rim/Neck Surface/Decorative Treatment

Rim/Neck/Body Thickness (cm)

Body Shape

Body Maximum Diameter $(\mathrm{cm})$

Body Height (cm)

Body Surface/Decorative Treatment

Base Shape/Curvature

Base Diameter/Height $(\mathrm{cm})$

Base Surface/Decorative Treatment

Appendages/Handles

Notes
8-26 / 3CL23

jar, tall rim

N3794

Military Road Incised

incised \& punctated

Bethel 1 (type) :: Bard 2 (type)

Compact, Hard, Smooth

Grog, medium \& abundant

$\operatorname{BLACK}(7.5 Y R 2.5 / 1)$

LIGHT BROWN (7.5YR6/3), DARK GRAY (7.5YR4/1)

BLACK (7.5YR2.5/1), LIGHT BROWN (7.5YR6/3)

16.5

703

15.8

Sooting on rim int \& ext; partial vessel, cat. as sherd lot, reconstructed, missing sherds from rim and body

JAR

Smoothed, beveled out, everted, 0.4

15.3

5.0

Straight, Outslanted

Smoothed, Incised, Punctated; double row vertical punctates, 5 horizontal meandering lines, double row vertical punctates

0.5

Sub-globular

15.8

11.5

Smoothed; Incised \& Punctated, meanding horizontal lines alternating with meandering double punctate row

Circular/Flat

7.5

Smoothed/Plain

Huddleston Grave 8, 1/14/1938[1939]; early Mid-Ouachita phase 
Artifact No. / Old Cat. No. / Site No.

Vessel Form

ARAS/HSU Digital Photo

Type

Decoration

Rim :: Body

Paste

Temper

Core Color (Munsell)

Exterior Color (Munsell)

Interior Color (Munsell)

Vessel Overall Height (cm)

Vessel Overall Weight (g)

Vessel Maximum Diameter (cm)

Volume (liters)

Usewear/Sooting/Condition

Shape/Description

Lip Treatment/Shape/Angle/Thickness

Orifice Exterior Diameter (cm)

Rim/Neck Height (cm)

Neck Mid/Base Exterior Diameter (cm)

Rim/Neck Shape/Angle

Rim/Neck Surface/Decorative Treatment

Rim/Neck/Body Thickness (cm)

Body Shape

Body Maximum Diameter $(\mathrm{cm})$

Body Height $(\mathrm{cm})$

Body Surface/Decorative Treatment

Base Shape/Curvature

Base Diameter/Height $(\mathrm{cm})$

Base Surface/Decorative Treatment

Appendages/Handles
8-27 / 3CL23

bowl, carinated

N3808

Friendship Engraved var. Meador

engraved

Elwyn 15 :: plain

Compact, Hard, Smooth, contains abundant mica

Grog, medium \& abundant

$\operatorname{RED}(2.5 \mathrm{YR} 5 / 6)$

DARK GRAY (10YR4/1), STRONG BROWN (7.5YR5/8), DARK GRAY (7.5YR4/1)

VERY DARK GRAY (10YR3/1), DARK GRAY (10YR4/1)

7.2

20.4

Partial vessel, reconstructed, missing sherds from lip, rim, body, base; no inked \#

Carinated Bowl

Burnished, flat, sl. everted, 0.3

20.4

4.2

Concave, Vertical

Burnished, engraved, ovals w/ ladders, crosshatching, no ticked line

0.5

Convex

20.4

3.0

Burnished; Plain

Undistinguished/Convex

Burnished/Plain

No information

Notes 
JEC Hodges Collection, 77-1

Artifact No. / Old Cat. No. / Site No.

Vessel Form

ARAS/HSU Digital Photo

Type

Decoration

Rim :: Body

Paste

Temper

Core Color (Munsell)

Exterior Color (Munsell)

Interior Color (Munsell)

Vessel Overall Height $(\mathrm{cm})$

Vessel Overall Weight (g)

Vessel Maximum Diameter $(\mathrm{cm})$

Volume (liters)

Usewear/Sooting/Condition

Shape/Description

Lip Treatment/Shape/Angle/Thickness

Orifice Exterior Diameter $(\mathrm{cm})$

Rim/Neck Height (cm)

Neck Mid/Base Exterior Diameter $(\mathrm{cm})$

Rim/Neck Shape/Angle

Rim/Neck Surface/Decorative Treatment

Rim/Neck/Body Thickness (cm)

Body Shape

Body Maximum Diameter $(\mathrm{cm})$

Body Height (cm)

Body Surface/Decorative Treatment

Base Shape/Curvature

Base Diameter/Height $(\mathrm{cm})$

Base Surface/Decorative Treatment

Appendages/Handles

Notes
8-28 / 1205 / 3CL23

bottle

1351

Keno Trailed var. Red Hill

incised

plain :: Belhaven 31

HARD, COMPACT

SHELL (LEACHED), FINE \& MED. ABUNDANT

BLACK, LIGHT GRAY, PALE BROWN

PALE BROWN

16.4

482

15.5

FIRECLOUDING

SQUAT GLOBULAR BOTTLE WITH PEDESTALLED BASE, SHORT NECK, FLARED LIP

ROUNDED, THINNED, EVERTED (FLARED)

5.9

4.7

BULBOUS / SPOOL

SMOOTHED \& BURNISHED, PLAIN

0.4

LOW-WAISTED

15.5

10.5

SMOOTHED \& BURNISHED; INCISED; NESTED INCISING, SQUARE ABOVE, SWIRL BELOW

PEDESTAL; CIRCULAR/FLAT

$7.0 / 1.0$

SMOOTHED \& BURNISHED, PLAIN

Huddleston Grave 6, 12/22/1938; Phillips photo 3917 (Huddleston 103); Social Hill-Deceiper phase 
JEC Hodges Collection, 77-1

Artifact No. / Old Cat. No. / Site No.

Vessel Form

ARAS/HSU Digital Photo

Type

Decoration

Rim :: Body

Paste

Temper

Core Color (Munsell)

Exterior Color (Munsell)

Interior Color (Munsell)

Vessel Overall Height $(\mathrm{cm})$

Vessel Overall Weight (g)

Vessel Maximum Diameter $(\mathrm{cm})$

Volume (liters)

Usewear/Sooting/Condition

Shape/Description

Lip Treatment/Shape/Angle/Thickness

Orifice Exterior Diameter $(\mathrm{cm})$

Rim/Neck Height (cm)

Neck Mid/Base Exterior Diameter (cm)

Rim/Neck Shape/Angle

Rim/Neck Surface/Decorative Treatment

Rim/Neck/Body Thickness (cm)

Body Shape

Body Maximum Diameter (cm)

Body Height $(\mathrm{cm})$

Body Surface/Decorative Treatment

Base Shape/Curvature

Base Diameter/Height $(\mathrm{cm})$

Base Surface/Decorative Treatment

Appendages/Handles

Notes
X-86 / 1211 / 3CL23

bowl, carinated

K5307

Friendship Engraved var. Freeman

Elaine 13 (type) :: plain

Compact, soft, smooth

Grog, medium \& abundant + Shell, leached, fine \& sparse

VERY PALE BROWN (10YR7/4), DARK GRAY (10YR4/1)

VERY DARK GRAY (10YR3/1)

10.0

979

26.0

Some roughness on base; fireclouding; partial vessel reassembled in past \& reglued; "1211" inked on side

bowl, carinated

Burnished, beveled, everted

26.0

5.0

Straight, vertical

Burnished, engraved, 2 tiers of scrolls and ladders separated by crosshatching

Convex

26.0

5.0

Burnished, plain

Undistinguished/Convex

Burnished, plain

Huddleston Grave 7, 1/14/1938[1939]; Phillips photo 3988 (Huddleston 118), Mid-Ouachita phase 
Artifact No. / Old Cat. No. / Site No.

Vessel Form

ARAS/HSU Digital Photo

Type

Decoration

Rim :: Body

Paste

Temper

Core Color (Munsell)

Exterior Color (Munsell)

Interior Color (Munsell)

Vessel Overall Height $(\mathrm{cm})$

Vessel Overall Weight (g)

Vessel Maximum Diameter (cm)

Volume (liters)

Usewear/Sooting/Condition

Shape/Description

Lip Treatment/Shape/Angle/Thickness

Orifice Exterior Diameter $(\mathrm{cm})$

Rim/Neck Height (cm)

Neck Mid/Base Exterior Diameter $(\mathrm{cm})$

Rim/Neck Shape/Angle

Rim/Neck Surface/Decorative Treatment

Rim/Neck/Body Thickness (cm)

Body Shape

Body Maximum Diameter $(\mathrm{cm})$

Body Height (cm)

Body Surface/Decorative Treatment

Base Shape/Curvature

Base Diameter/Height $(\mathrm{cm})$

Base Surface/Decorative Treatment

Appendages/Handles

Notes
22-1 / 2806 / 3HS103

jar, tall rim

K8402

incised

Beloit 10 :: Abraham 6

Compact, hard, gritty, mica in paste

Shell, leached shell, abundant \& coarse

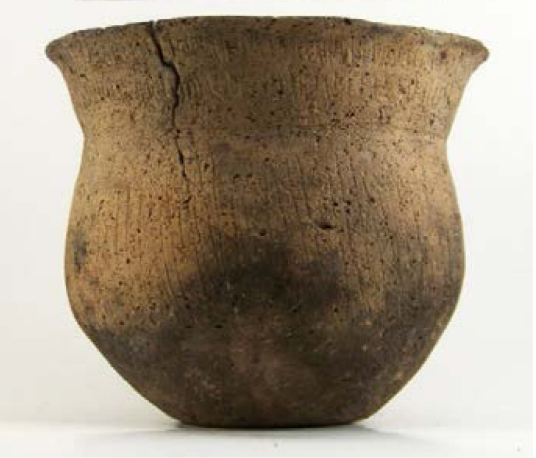

PINKISH GRAY (7.5YR6/2-7/2), GRAY (7.5YR5/1)

GRAY (7.5YR5/1)

13.1

300

13.8

1.1

Overall erosion (post-depositional); remnant of sooting on interior neck; vessel mended in past; "2806" inked on int rim

Jar, Tall Rim

smoothed, rounded, sl. everted, 0.4

13.1

3.5

Concave, outslanted

Smoothed, incised, 2 rows of vertical lines $4 \mathrm{~mm}$ apart, horizontal line at rim/body junction

0.5

Sub globular

12.0

9.6

Smoothed; Incised, vertical lines to $4 \mathrm{~cm}$ above base, $3-5 \mathrm{~mm}$ apart

Circular/Flat

6.1

Smoothed/Plain

Huddleston Burial 1, 5/29/1943; Social Hill phase 
Artifact No. / Old Cat. No. / Site No.

Vessel Form

ARAS/HSU Digital Photo

Type

Decoration

Rim :: Body

Paste

Temper

Core Color (Munsell)

Exterior Color (Munsell)

Interior Color (Munsell)

Vessel Overall Height (cm)

Vessel Overall Weight (g)

Vessel Maximum Diameter (cm)

Volume (liters)

Usewear/Sooting/Condition

Shape/Description

Lip Treatment/Shape/Angle/Thickness Orifice Exterior Diameter (cm)

Rim/Neck Height (cm)

Neck Mid/Base Exterior Diameter (cm)

Rim/Neck Shape/Angle

Rim/Neck Surface/Decorative Treatment

Rim/Neck/Body Thickness (cm)

Body Shape

Body Maximum Diameter (cm)

Body Height $(\mathrm{cm})$

Body Surface/Decorative Treatment

Base Shape/Curvature

Base Diameter/Height $(\mathrm{cm})$

Base Surface/Decorative Treatment

Appendages/Handles

Notes
22-2 / 2804 / 3HS103

jar, short rim

1392

incised

Albertus 7 :: Abraham 12

HARD, FINE, COMPACT

SHELL (LEACHED), ABUNDANT, MEDIUM

DARK GRAY

PINK, VERY DARK GRAY

PINK, VERY DARK GRAY

8.3

116

8.3

FIRECLOUDING; RIM BROKEN AWAY ON ONE SIDE

SMALL JAR; FLAT BASE; SUBGLOBULAR BODY; FLARED RIM

CONCAVE, EVERTED (FLARED)

8.3

2.8

CONCAVE, OUTSLANTED

SMOOTHED; INCISED, DIAGONAL INCISED LINES, SPACED

$0.5-0.6$

SUBGLOBULAR

7.5

5.5

SMOOTHED; VERTICAL INCISING, SPACED, ON UPPER 2/3 OF BODY

CIRCULAR/FLAT

3.1

Huddleston Burial 1, 5/29/1943; Social Hill phase 
JEC Hodges Collection, 77-1

Artifact No. / Old Cat. No. / Site No.

Vessel Form

ARAS/HSU Digital Photo

Type

Decoration

Rim :: Body

Paste

Temper

Core Color (Munsell)

Exterior Color (Munsell)

Interior Color (Munsell)

Vessel Overall Height (cm)

Vessel Overall Weight (g)

Vessel Maximum Diameter $(\mathrm{cm})$

Volume (liters)

Usewear/Sooting/Condition

Shape/Description

Lip Treatment/Shape/Angle/Thickness

Orifice Exterior Diameter $(\mathrm{cm})$

Rim/Neck Height (cm)

Neck Mid/Base Exterior Diameter (cm)

Rim/Neck Shape/Angle

Rim/Neck Surface/Decorative Treatment

Rim/Neck/Body Thickness (cm)

Body Shape

Body Maximum Diameter (cm)

Body Height $(\mathrm{cm})$

Body Surface/Decorative Treatment

Base Shape/Curvature

Base Diameter/Height (cm)

Base Surface/Decorative Treatment

Appendages/Handles

Notes
$22-3 / 2808$ / 3HS103

bowl, carinated

K5456

Sandford Punctated

punctated

Concord 1 :: plain

Compact, smooth

Shell, leached, abundant \& medium

DARK REDDISH GRAY (5YR4/2), PINKISH GRAY (5YR6/2)

DARK GRAY (5YR4/1)

8.5

324

21.0

1.6

Partial vessel, reconstructed in past, possible probe hole near base; fireclouding

Bowl, Carinated

smoothed, rounded, none, 0.5

19.5

2.5

Convex, inslanted

Smoothed, Punctated, 3 horizontal rows of fingernail punctates forming ridging 0.5

Convex

21.0

6.0

Smoothed; plain

undistinguished/convex

Smoothed/Plain

Huddleston Burial 1, 5/29/1943; Social Hill phase 
Artifact No. / Old Cat. No. / Site No.

Vessel Form

ARAS/HSU Digital Photo

Type

Decoration

Rim :: Body

Paste

Temper

Core Color (Munsell)

Exterior Color (Munsell)

Interior Color (Munsell)

Vessel Overall Height (cm)

Vessel Overall Weight (g)

Vessel Maximum Diameter $(\mathrm{cm})$

Volume (liters)

Usewear/Sooting/Condition

Shape/Description

Lip Treatment/Shape/Angle/Thickness

Orifice Exterior Diameter $(\mathrm{cm})$

Rim/Neck Height (cm)

Neck Mid/Base Exterior Diameter $(\mathrm{cm})$

Rim/Neck Shape/Angle

Rim/Neck Surface/Decorative Treatment

Rim/Neck/Body Thickness (cm)

Body Shape

Body Maximum Diameter (cm)

Body Height (cm)

Body Surface/Decorative Treatment

Base Shape/Curvature

Base Diameter/Height $(\mathrm{cm})$

Base Surface/Decorative Treatment

Appendages/Handles

Notes
22-4 / 2805 / 3HS103

bottle

K5748

Blakely Engraved var. Witherspoon

engraved

Elgin 1 :: Emil 3

Compact, Hard, Smooth, mica

Shell, leached, fine \& sparse - may have sparse fine grog also

LIGHT RED (2.5YR6/8)

GRAY (7.5YR5/1), LIGHT REDDISH BROWN (5YR6/4)

20.8

635

14.8

1.0

Wear on base?; fireclouding; intact vessel, chip in lip

Bottle

smoothed, rounded, sl. everted, 0.4

4.0

8.0

$3.8 / 5.2$

Vertical, slight spool

Burnished

0.5

globular

14.8

12.6

Burnished; Engraved; 1 horizontal ticked line at base of neck, alternating two vertical lines and three lines with $1 / 2$ diamond $\mathrm{x} 2$; trace red ( $\&$ white?) pigment in engraving

circular/flat

6.0

Burnished/Plain

Huddleston Burial 1, 5/29/1943; Social Hill phase

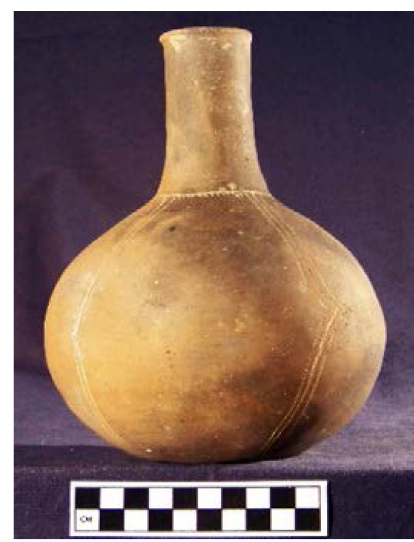




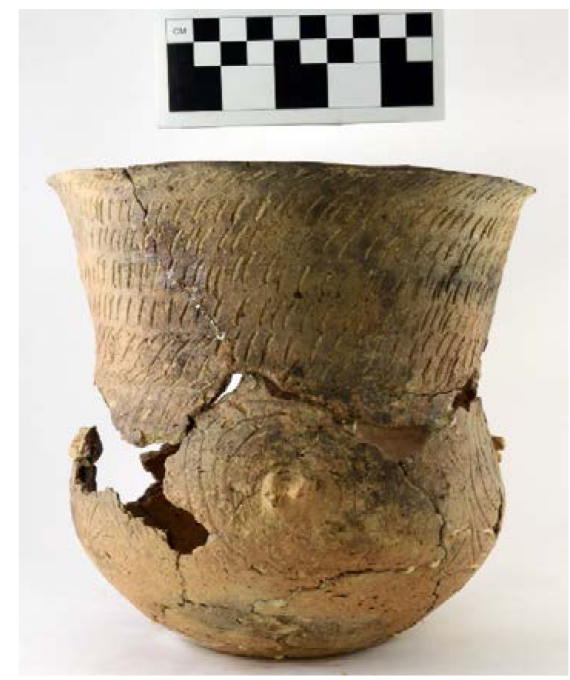

Artifact No. / Old Cat. No. / Site No.

Vessel Form

ARAS/HSU Digital Photo

Type

Decoration

Rim :: Body

Paste

Temper

Core Color (Munsell)

Exterior Color (Munsell)

Interior Color (Munsell)

Vessel Overall Height (cm)

Vessel Overall Weight (g)

Vessel Maximum Diameter (cm)

Volume (liters)

Usewear/Sooting/Condition

Shape/Description

Lip Treatment/Shape/Angle/Thickness

Orifice Exterior Diameter (cm)

Rim/Neck Height (cm)

Neck Mid/Base Exterior Diameter (cm)

Rim/Neck Shape/Angle

Rim/Neck Surface/Decorative Treatment

Rim/Neck/Body Thickness (cm)

Body Shape

Body Maximum Diameter $(\mathrm{cm})$

Body Height (cm)

Body Surface/Decorative Treatment

Base Shape/Curvature

Base Diameter/Height $(\mathrm{cm})$

Base Surface/Decorative Treatment

Appendages/Handles

Notes
22-5 / 2807 / 3HS103

jar, tall rim

N11334

Foster Trailed-Incised

incised \& punctated

Chattanooga 1 :: Baker 20

Crumbly, Soft, Smooth

Shell, leached, coarse and abundant

VERY DARK GRAY (7.5YR3/1)

GRAY (7.5YR5/1), PINKISH GRAY (7.5YR7/2), VERY DARK GRAY (10YR3/1)

BROWN (7.5YR4/3), DARK GRAY (7.5YR4/1)

20.0

778

19.5

Fireclouding; partial vessel, reconstructed in past, large portions of body missing; 2807 inked on base

Jar, Tall Rim

smoothed, rounded, sl. everted, 0.3

19.5

9.4

Concave, outslanted

Smoothed, Punctated, 8 rows of diagonal punctates, almost vertical

$0.4-0.5$

Subglobular

17.6

10.6

Smoothed; Incised, concentric incised circles (ends $4 \mathrm{~cm}$ above base), $3 \mathrm{pt}$ nodes in center of each, $\mathrm{x} 4$

Circular/Flat

4.7

Smoothed/Plain

4 tri-part nodes on body

Huddleston Burial 1, 5/29/1943; Social Hill phase 
JEC Hodges Collection, 77-1

Artifact No. / Old Cat. No. / Site No.

Vessel Form

ARAS/HSU Digital Photo

Type

Decoration

Rim :: Body

Paste

Temper

Core Color (Munsell)

Exterior Color (Munsell)

Interior Color (Munsell)

Vessel Overall Height (cm)

Vessel Overall Weight (g)

Vessel Maximum Diameter $(\mathrm{cm})$

Volume (liters)

Usewear/Sooting/Condition

Shape/Description

Lip Treatment/Shape/Angle/Thickness

Orifice Exterior Diameter $(\mathrm{cm})$

Rim/Neck Height (cm)

Neck Mid/Base Exterior Diameter (cm)

Rim/Neck Shape/Angle

Rim/Neck Surface/Decorative Treatment

Rim/Neck/Body Thickness (cm)

Body Shape

Body Maximum Diameter (cm)

Body Height (cm)

Body Surface/Decorative Treatment

Base Shape/Curvature

Base Diameter/Height (cm)

Base Surface/Decorative Treatment

Appendages/Handles

Notes
29-1 / 2467 / 3CL26

seed jar

N15539

plain

Compact, Hard, Smooth, mica

Shell, leached + grog, coarse \& abundant

YELLOWISH RED (5YR4/6) (CORE MODE A)

RED (2.5YR5/6), REDDISH BROWN (5YR5/4), GRAY (5YR5/1)

24.5

1109

16.5

Fireclouding; partial vessel, reconstructed in past, sherds missing/unattached from body/base; 2467 inked on lower body

Seed Jar

eroded, flat/beveled in, none, 0.6

3.5

Convex, inslanted (continuous with body)

Eroded, plain

0.7

Barrel

16.5

24.5

Eroded (patches of original burnishing); Plain

Circular/Flat

14.5

eroded

Huddleston Burial Y, 2/23/1941; Mid-Ouachita phase 
Artifact No. / Old Cat. No. / Site No.

Vessel Form

ARAS/HSU Digital Photo

Type

Decoration

Rim :: Body

Paste

Temper

Core Color (Munsell)

Exterior Color (Munsell)

Interior Color (Munsell)

Vessel Overall Height $(\mathrm{cm})$

Vessel Overall Weight (g)

Vessel Maximum Diameter $(\mathrm{cm})$

Volume (liters)

Usewear/Sooting/Condition

Shape/Description

Lip Treatment/Shape/Angle/Thickness

Orifice Exterior Diameter $(\mathrm{cm})$

Rim/Neck Height (cm)

Neck Mid/Base Exterior Diameter $(\mathrm{cm})$

Rim/Neck Shape/Angle

Rim/Neck Surface/Decorative Treatment

Rim/Neck/Body Thickness (cm)

Body Shape

Body Maximum Diameter (cm)

Body Height (cm)

Body Surface/Decorative Treatment

Base Shape/Curvature

Base Diameter/Height $(\mathrm{cm})$

Base Surface/Decorative Treatment

Appendages/Handles

Notes
29-2 / 2464 / 3CL26

bottle

429, N15509

Blakely Engraved var. Witherspoon

engraved

plain :: Emil 6

Compact, Hard, Smooth

Shell, leached, fine \& abundant + grog, fine

VERY DARK GRAY (5YR3/1) (CORE MODE F)

PINKISH GRAY (7.5YR7/2), VERY DARK GRAY (5YR3/1), PINK (7.5YR7/3)

BROWN (7.5YR5/3)

15.0

281

9.5

0.3

Eroded surfaces on neck/body; fireclouding; intact vessel, minor chips in lip; 2464 inked on side

SMALL BOTTLE-LONG STRAIGHT NECK, GLOBULAR BODY, FLAT BASE

Burnished, round, sl. everted, 0.3

4.3

7.0

$4.3 / 4.6$

Vertical

Burnished, plain

0.4

Globular

9.3

8.0

Burnished (mostly eroded); Engraved, 3 groups of 3 vertical lines (ends around $1.5 \mathrm{~cm}$ above base)

Circular/Flat

5.0

Burnished/Plain

Huddleston Burial X, 2/23/1941; Mid-Ouachita - Social Hill phase 
Artifact No. / Old Cat. No. / Site No.

Vessel Form

ARAS/HSU Digital Photo

Type

Decoration

Rim :: Body

Paste

Temper

Core Color (Munsell)

Exterior Color (Munsell)

Interior Color (Munsell)

Vessel Overall Height $(\mathrm{cm})$

Vessel Overall Weight $(\mathrm{g})$

Vessel Maximum Diameter $(\mathrm{cm})$

Volume (liters)

Usewear/Sooting/Condition

Shape/Description

Lip Treatment/Shape/Angle/Thickness

Orifice Exterior Diameter $(\mathrm{cm})$

Rim/Neck Height (cm)

Neck Mid/Base Exterior Diameter $(\mathrm{cm})$

Rim/Neck Shape/Angle

Rim/Neck Surface/Decorative Treatment

Rim/Neck/Body Thickness (cm)

Body Shape

Body Maximum Diameter (cm)

Body Height (cm)

Body Surface/Decorative Treatment

Base Shape/Curvature

Base Diameter/Height (cm)

Base Surface/Decorative Treatment

Appendages/Handles
29-3 / 2465 / 3CL26

jar, short rim

N15456

incised

Andes 4 :: Bard 8 or Altus 2

Crumbly, Soft, Smooth

Shell, leached, coarse and abundant

BLACK (7.5YR2.5/1) (CORE MODE B)

LIGHT BROWN (7.5YR6/3), PINKISH GRAY (7.5YR7/2), DARK GRAY (7.5YR4/1)

DARK GRAY (7.5YR4/1)

10.5

213

12.8

Soot on interior rim and upper body, trace on body exterior, fireclouding; partial vessel, missing $1 / 2$ rim and 1/4 body (some sherds unattached in bag); 2465 inked on bottom

Jar, Short Rim

Smoothed, round, everted, 0.4

12.6

2.5

Concave, outslanted

Smoothed, Incised, 2 rows of diagonal slash marks in opposite directions

0.4

Globular

12.8

8.0

Smoothed; Incised, 2 rows of digonal slashes in opposite directions, above 9 horizontal lines around mid-body

Circular/Flat

6.0

Smoothed/Plain

Huddleston Burial X, 2/23/1941; Mid-Ouachita - Social Hill phase 